\title{
REFERENCE
}

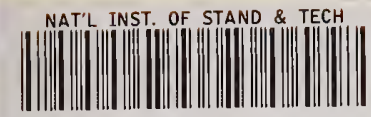

Al1105 96910ट

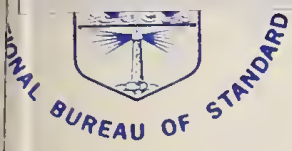

NBS SPECIAL PUBLICATION 412

U.S. DEPARTMENT OF COMMERCE / National Bureau of Standards

\section{Aerosol Measurements}

$=Q C$
100
.457
19.412
1974


The National Bureau of Standards ${ }^{1}$ was established by an act of Congress March 3, 1901. The Bureau's overall goal is to strengthen and advance the Nation's science and technology and facilitate their effective application for public benefit. To this end, the Bureau conducts research and provides: (1) a basis for the Nation's physical measurement system, (2) scientific and technological services for industry and government, (3) a technical basis for equity in trade, and (4) technical services to promote public safety. The Bureau consists of the Institute for Basic Standards, the Institute for Materials Research, the Institute for Applied Technology, the Institute for Computer Sciences and Technology, and the Office for Information Programs.

THE INSTITUTE FOR BASIC STANDARDS provides the central basis within the United States of a complete and consistent system of physical measurement; coordinates that system with measurement systems of other nations; and furnishes essential services leading to accurate and uniform physical measurements throughout the Nation's scientific community, industry, and commerce. The Institute consists of a Center for Radiation Research, an Office of Measurement Services and the following divisions:

Applied Mathematics - Electricity - Mechanics - Heat - Optical Physics - Nuclear Sciences $^{2}$ - Applied Radiation ${ }^{2}$ - Quantum Electronics ${ }^{3}$ - Electromagnetics ${ }^{3}$ - Time and Frequency ${ }^{3}$ - Laboratory Astrophysics ${ }^{3}$ - Cryogenics ${ }^{3}$.

THE INSTITUTE FOR MATERIALS RESEARCH conducts materials research leading to improved methods of measurement, standards, and data on the properties of well-characterized materials needed by industry, commerce, educational institutions, and Government; provides advisory and research services to other Government agencies; and develops, produces, and distributes standard reference materials. The Institute consists of the Office of Standard Reference Materials and the following divisions:

Analytical Chemistry - Polymers - Metallurgy — Inorganic Materials — Reactor Radiation - Physical Chemistry.

THE INSTITUTE FOR APPLIED TECHNOLOGY provides technical services to promote the use of available technology and to facilitate technological innovation in industry and Government; cooperates with public and private organizations leading to the development of technological standards (including mandatory safety standards), codes and methods of test; and provides technical advice and services to Government agencies upon request. The Institute consists of a Center for Building Technology and the following divisions and offices:

Engineering and Product Standards - Weights and Measures - Invention and Innovation - Product Evaluation Technology - Electronic Technology - Technical Analysis

- Measurement Engineering - Structures, Materials, and Life Safety * - Building

Environment * - Technical Evaluation and Application * Fire Technology.

THE INSTITUTE FOR COMPUTER SCIENCES AND TECHNOLOGY conducts research and provides technical services designed to aid Government agencies in improving cost effectiveness in the conduct of their programs through the selection, acquisition, and effective utilization of automatic data processing equipment; and serves as the principal focus within the executive branch for the development of Federal standards for automatic data processing equipment, techniques, and computer languages. The Institute consists of the following divisions:

Computer Services - Systems and Software - Computer Systems Engineering - Information Technology.

THE OFFICE FOR INFORMATION PROGRAMS promotes optimum dissemination and accessibility of scientific information generated within NBS and other agencies of the Federal Government; promotes the development of the National Standard Reference Data System and a system of information analysis centers dealing with the broader aspects of the National Measurement System; provides appropriate services to ensure that the NBS staff has optimum accessibility to the scientific information of the world. The Office consists of the following organizational units:

Office of Standard Reference Data - Office of Information Activities - Office of Technical Publications - Library - Office of International Relations.

\footnotetext{
${ }^{1}$ Headquarters and Laboratories at Gaithersburg, Maryland, unless otherwise noted; mailing address Washington, D.C. 20234.

2 Part of the Center for Radiation Research.

${ }^{3}$ Located at Boulder, Colorado 80302.

- Part of the Center for Building Technology.
} 
221974

tacc-Ref.

\section{AEROSOL MEASUREMENTS}

The Proceedings of a Seminar on

Aerosol Measurements

May 7, 1974

Wayne A. Cassatt and

Rosemary S. Maddock, editors

Analytical Chemistry Division Institute for Materials : Research

U.S. National Bureau of Standards

$\cdots \quad$ Washington, D. C. 20234

\section{t. special publication no, 412}

\section{Sponsored by}

The National Bureau of Standards

Washington, D. C. 20234

and

The Food and Drug Administration

Washington, D. C. 20204

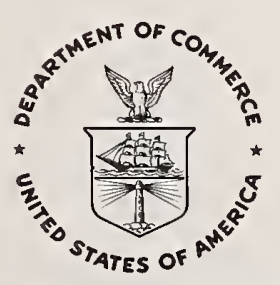

\section{U.S. DEPARTMENT OF COMMERCE, Frederick B. Dent, Secrefary}

NATIONAL BUREAU OF STANDARDS, Richard W. Roberts, Director

Issued October 1974 
Library of Congress Catalog Number: 74-600164

National Bureau of Standards Special Publication 412

Nat. Bur. Stand. (U.S.), Spec. Publ. 412, 193 pages (Oct. 1974)

CODEN: XNBSAV 
Papers followed by verbal discussions were given in a seminar and workshop sponsored by the National Bureau of Standards and the Food and Drug Administration to define the state of development of aerosol measuring instruments. The instruments discussed were based upon a variety of operating principles including laser light scattering, optical imaging, Doppler shift, electromobility, piezoelectric effect, and beta-ray absorption. Two review papers were given which described other phenomena upon which aerosol measurements are based. The general summary includes a table which lists the specifications of the instruments discussed to illustrate the range of capabilities available in this field. Discussion among seminar attendees revealed that many questions remain to be answered before the more difficult aerosol measurements problems can be solved. For example, in the analysis of very dense aerosols questions arise concerning coincidence losses or agglomeration effects that may result from collisions between particles as they are drawn into the measuring volume. Volatilization or condensation effects may alter the size distribution if the measurements are made late in time. Finally, variations in particle shape or index of refraction can alter the instrument response and cause difficulties in interpretation.

Key Words: Aerosol instrument performance; aerosol measuring instruments; beta-ray absorption; Doppler shift; electromobility; laser light scattering; optical imaging; piezoelectric effect 
The use of aerosols has found wide popularity in most life styles of our population. Toxic aerosols present special problems which require accurate measurement of airborne particles and droplets so that their effect on health can be evaluated.

Meaningful monitoring of aerosol particles usually must be performed in real time and at the site of exposure. Rigorous and exacting measurement conditions must be met. Thus, new and innovative methods and instrumentation are being developed to meet the rigorous monitoring criteria necessary to safeguard health.

The dramatic increase in the number of food, drug and cosmetic products now consumed as aerosol spray products coupled with their measurement needs have prompted the Food and Drug Administration and the National Bureau of Standards to co-sponsor this Seminar on Aerosol Measurements to better define the needs and to stimulate the development of new methodology for making rapid and reliable aerosol measurements.

Philip D. LaFleur, Acting Chief Analytical Chemistry Division 


\section{PREFACE}

This seminar was organized to provide an opportunity for representatives of instrument manufacturers, research groups and government agencies to evaluate the latest developments in instrumentation for making rapid measurements on aerosols. The incentive behind this effort is the realization that the popular uses of aerosols and the accompanying exposures are rapidly rising in frequency and importance. Over two billion spray cans are used each year to apply paints, insecticides, deodorants, oven cleaners and a host of other useful but toxic materials. The need to maintain quality control in spray applications and to monitor any potential hazards demands an ability to measure aerosol particles accurately and reproducibly.

The particle sizes and concentrations encountered in aerosol sprays place such unusual demands on the measuring techniques that conventional instruments appear limited in their ability to provide the required dynamic range, resolution and accuracy. This workshop was directed, therefore, to a discussion of a representative selection of the newer aerosol instruments that are under development or have been completed recently.

It is hoped that these proceedings will serve both to illustrate some of the latest approaches to aerosol instrument design and to stimulate the development of additional new concepts in the future.

Wayne A. Cassatt, Coordinator

Seminar on Aerosol Measurements 
Abstract . . . . . . . . . . . . . . .

Foreword . . . . . . . . . . . . . . . . . . .

A Review of the Methods for the Particle Size Analysis

of Aerosol Spray Can Droplets . . . . . . . . . .

R. Davies and J. D. Stockham

Light Scattering by Single Aerosol Particles . . . . . . . Milton Kerker

Light Scattering Methods for the Characterization of Particulate Matter in Real Time . . . . . . . . .

C. C. Gravatt

Flow Apparatus for the Characterization of Aerosols . . . Madhav B. Ranade

$360^{\circ}$ Scattering Diagrams from Individual Aerosols in a Flowing Stream

Thomas R. Marshal1, Charles S. Parmenter, and Mark Seaver

Active Scattering Aerosol Spectrometry . . . . . . . .

Robert G. Knollenberg

Rapid Measurement of Droplet Size Distributions by

Optical Heterodyne Spectroscopy ...........

Ilan Chabay

Measurements of Aerosol Size Distributions with a

Laser Doppler Velocimeter (LDV) . . . . . . . . .

William J. Yanta 
An Optical Transform Technique for Measuring the

Size Distribution of Particles in Fluids . . . . . . . .

Albert McSweeney

Sizing Aerosols in Real Time by Pulsing UV Laser

Machine . . . . . . . . . . . . . . .

Geoffrey A. Hotham

Rapid Respirable Mass Measurement . . . . . . . . . .

Lawrence Doemeny, George Carson, and Bruce Almich

Particulate Mass Measurement by Piezoelectric

Crystal ...................

Raymond L. Chuan

Development, Calibration and Application of Size

Distribution Instruments at the University of

Minnesota ..................

V. A. Marple

List of Attendees . . . . . . . . . . . . . .

Authors Index ....................

Key Word Index . . . . . . . . . . . . . . . 
The papers recorded in these proceedings describe several aerosol measuring instruments based upon a variety of operating principles. The physical phenomenon underlying these principles include' laser light scattering, optical imaging, Doppler shift, electromobility, piezoelectric effect, and beta-ray absorption. Two review papers are included which describe several other phenomena upon which measurements are based. Instruments designed around the first three principles have capabilities for detecting and sizing indivdual aerosol particles, but they have definite limitations regarding the concentrations and count rates that they can handle. Instruments based upon the latter three principles are much more adept at handling high concentrations but it is difficult, if not impossible, to detect and to size individual particles with them. Obviously, the principle to be employed and the specific design of the final instrument will depend upon the measurement requirements to be met. Those devices that rely upon the interaction between light and individual particles can be quite different in basic design and performance. Some measure intensity at one or two selected angles, others analyze the intensity pattern over large solid angles, and still others measure focused optical images. In a11, some eight different instruments of this type were discussed here.

In order to compare these instruments and their performances in a convenient fashion, they have been listed along with their specifications in table ?. This is by no means intended to be a complete list of the aerosol instruments on the market or under construction in various laboratories around the country. Rather, it is meant to be indicative of the state of the development in this field.

Discussions among seminar attendees revealed that many questions remain to be answered before the more difficult aerosol measurement problems can be solved. For example, in the analys is of very dense aerosols questions arise concerning coincidence losses or agglomeration effects that may result from collisions between particles as they are drawn into the measuring volume. Volatilization or condensation effects may alter the size distribution if the measurements are made late in time. the lack of size resolution or sensitivity may prevent the detection of useful information. In some cases the lack of instrument portability or the length of time required for data processing may constitute serious handicaps. Finally, variations in particle shape or index of refraction can alter the instrument response and cause difficulties in interpretation.

The developers of the instruments described in this seminar have quite successfully attacked and solved one or more of these problems with respect to aerosol spray measurements. On the other hand, it would appear that much remains to be done to produce an optimum device, especially if it is to be useful for general applications under a variety of conditions.

Wayne A. Cassatt, Coordinator Seminar on Aerosol Measurements 


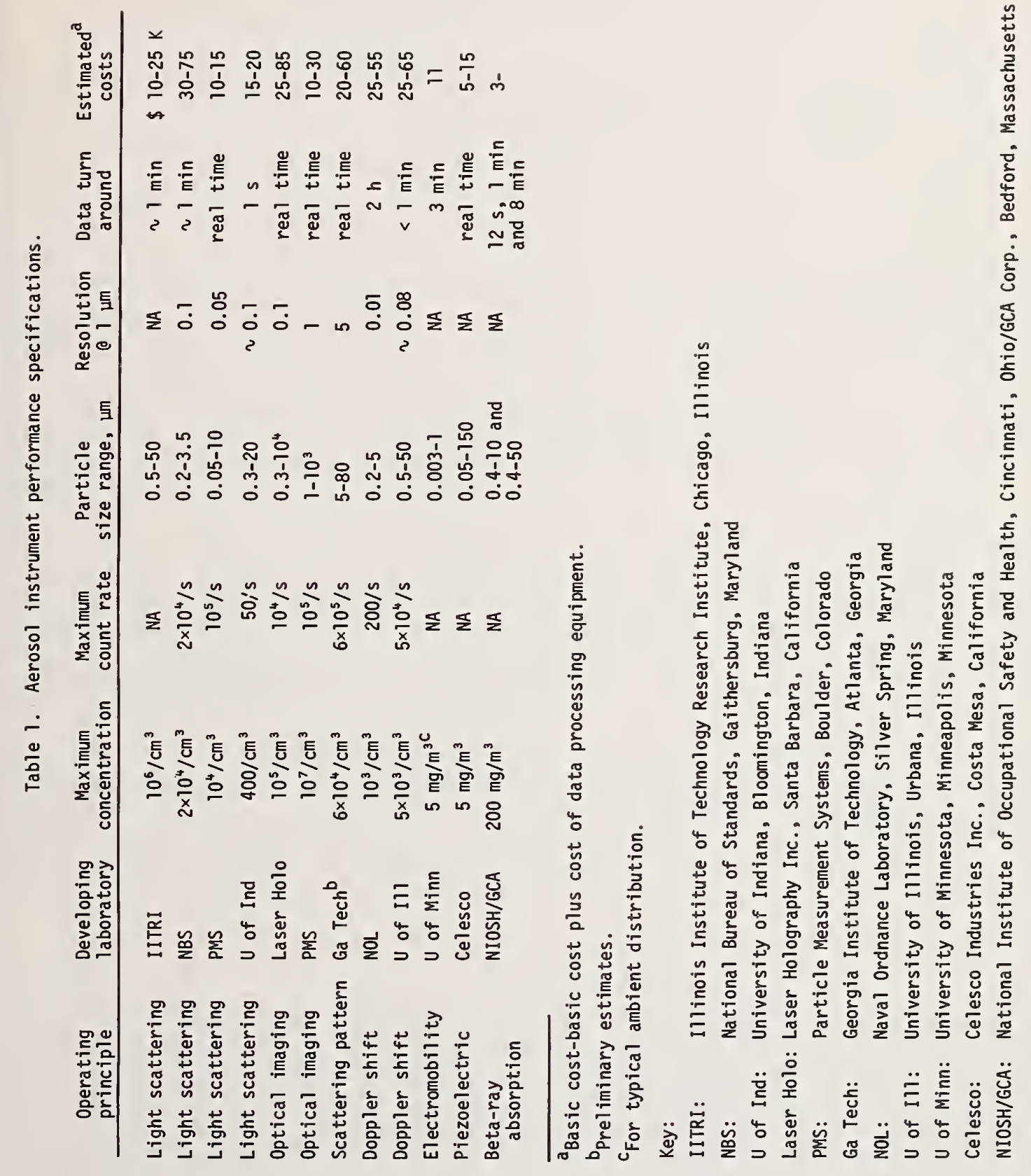





\title{
A REVIEW OF THE METHODS FOR THE PARTICLE SIZE ANALYSIS OF AEROSOL SPRAY CAN DROPLETS
}

\author{
R. Davies and J. D. Stockham \\ IIT Research Institute \\ 10 West 35 th Street \\ Chicago, Illinois 60616
}

\section{ABSTRACT}

This paper reviews the methods that have been used for sizing droplets, sprays, and mists, but places particular emphasis on the methods that are applicable to the sizing of aerosol spray can droplets.

The methods reviewed include photographic imaging, collection, deposition and size reconstruction, momentum transfer, pulse counting, hot wire anemometry, electrical mobility, optical scattering, and interference.

From the various techniques discussed in each class, laser holographic imaging is considered the best approach, but the method has an extremely high capital cost. Light scattering methods are the second choice, but these, too, have a relatively high capital cost. If capital cost is an important factor, then methods such as photographic imaging and impaction can be usefully employed.

Key words: aerosol instrumentation; aerosol measurements; aerosol review; aerosols; aerosol scattering; aerosol sizing; droplet sizing; droplet imaging; electrical mobility; impaction; momentum methods; particle imaging; particle measurements; particle scattering; particle size determinations; particulates; sprays.

\section{INTRODUCTION}

In 1973, 1,900 million aerosol can products were produced in Europe, and the U.S.A. contribution to atmospheric pollution from spray can sources was estimated to be 5.4 ton/day/106 population [1]. Though the total emission from this source only represents a minor source of pollution, the nature of the emission has recently caused some concern. Reportedly, the fluorinated hydrocarbons used as a propellant have produced toxic effects on the hearts of animals [2] and vinyl chloride, used in some products, has caused cancer of the liver [3]. Also, with the advent of the Occupational Safety and Health Administration (OSHA) regulations, the inhalation hazard of particulates from spray can 
products is now coming under scrutiny, and such agencies as the EPA, NIOSH, and FDA are taking a close look at aerosolized product effects. However, to determine the effect of these particulates, vapors, and gases on animals during controlled experiments, and to predict their effect on human life, the physical properties of the spray have to be known. One important physical property of the spray that controls its deposition behavior in the lungs is the particle size and size distribution. In consequence, it is the purpose of this paper to briefly review the methods that have been used successfully to measure the particle size and size distribution of spray in general. From this review, useful methods that can be employed for spray can analys is are suggested.

The information presented here has been abstracted from a 200 reference article presently being prepared by the author for publication elsewhere [4]. In addition, some material has been drawn from the series of four articles published by the author, on "Rapid Response Methods of Size Analysis" [5-8].

\section{METHODS OF MEASURING DROPLET SIZE}

Generally, the methods can be grouped in the categories below.

1. photographic imaging methods

2. collection and deposition methods

3. momentum transfer methods

4. pulse counting methods

5. hot-wire anemometry methods

6. electrical mobility methods

7. optical methods

\subsection{Photographic Imaging}

Photographic methods were first used by Mache in Germany at the turn of the century. Recent uses of the method have been by Mueller and Sims [9] and Itagaki and Meline [10]. Meline used a 4" $\times 5^{\prime \prime}$ press camera with a long focal length and large shutter aperture, producing a sharp plane of focus in the droplet field.

Webster [11] used imaging systems to record high velocity droplets in flight. Similar systems have been used by Knollenberg [12], and Cannon [13]. The latter author used the method for measuring cloud droplets. Stockham, et al. [14] developed an automatic spray analyzer for Holloman Air Force Base, which imaged the droplets on a TV tube (fig. 1a). A comparison with conventional photographic methods was shown to be good (fig. 1b). A similar system was developed by Spraying Systems Inc. [15].

The method is more fully discussed in references 4 and 5 . 


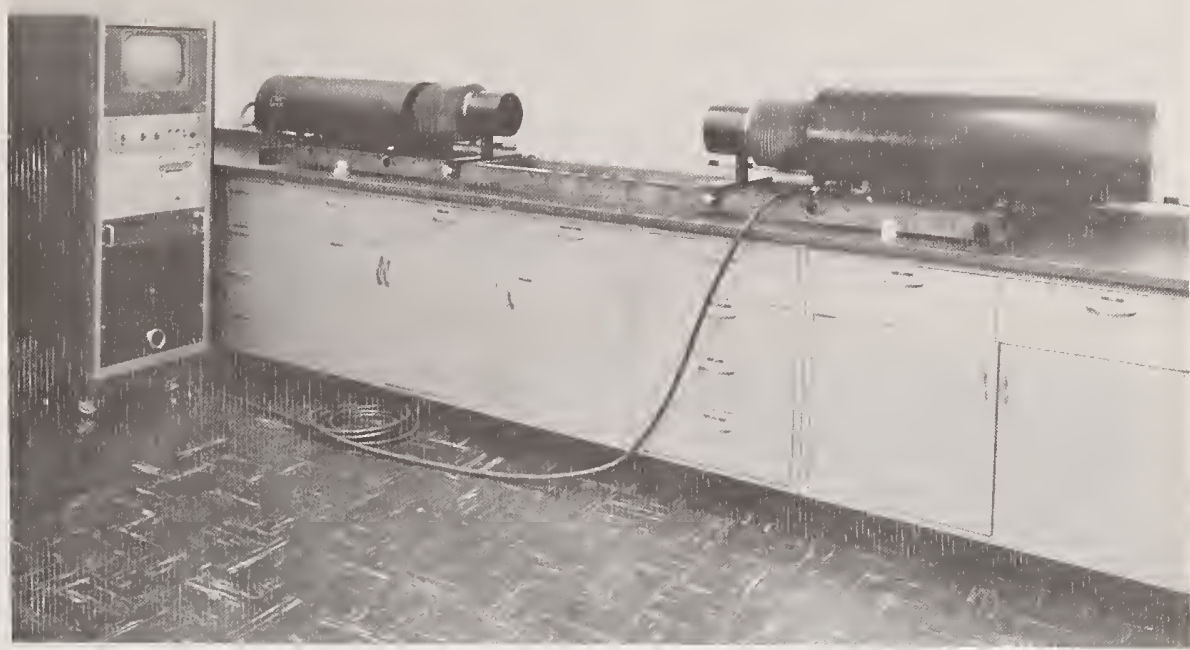

b

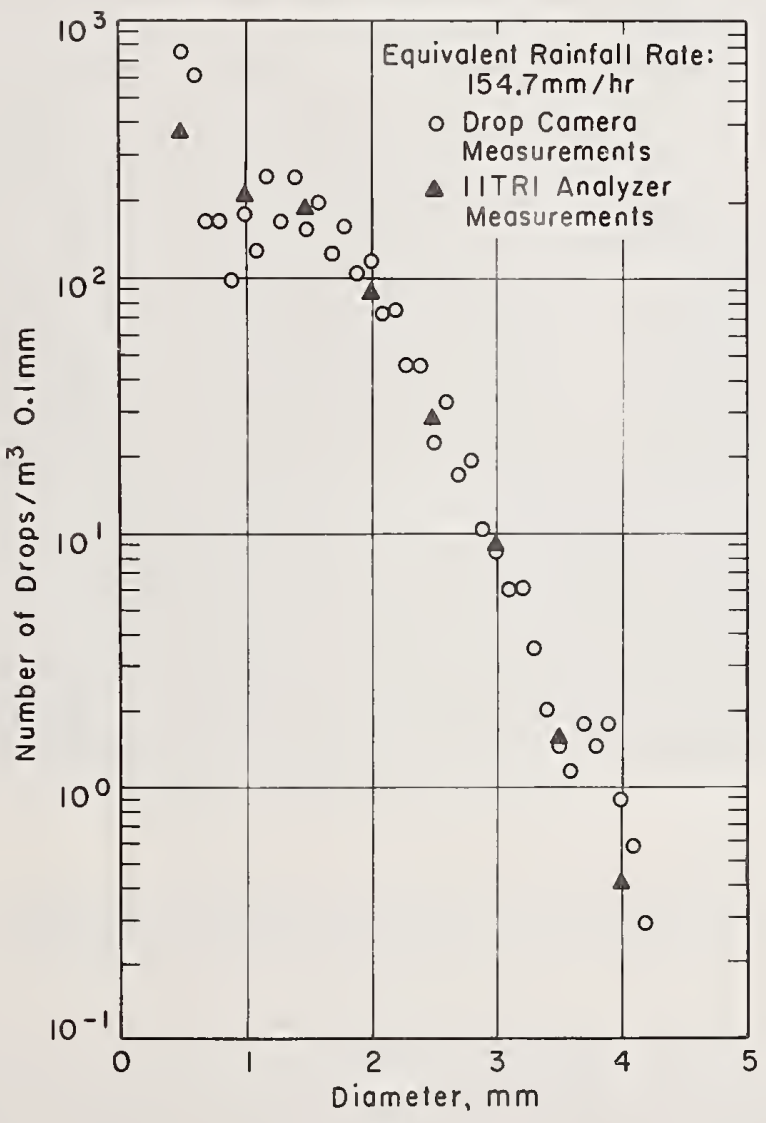

Figure 1. a. Photograph of spray droplet analyzer.

b. Data reported by Stockham et az. [14]. 


\subsection{Collection Methods}

In these methods, the particles are removed from the fluid by various physical mechanisms and deposited onto surfaces or into immiscible liquids. Sizing is then performed on the deposit. Separating mechanisms have included gravity and centrifugal sedimentation, horizontal elutriation, impaction, thermal and electrostatic precipitation. An example of a centrifugal collector is the Goetz Spectrometer (fig. 2), and an example of a liquid droplet impactor is the Brink impactor (fig. 3 ).

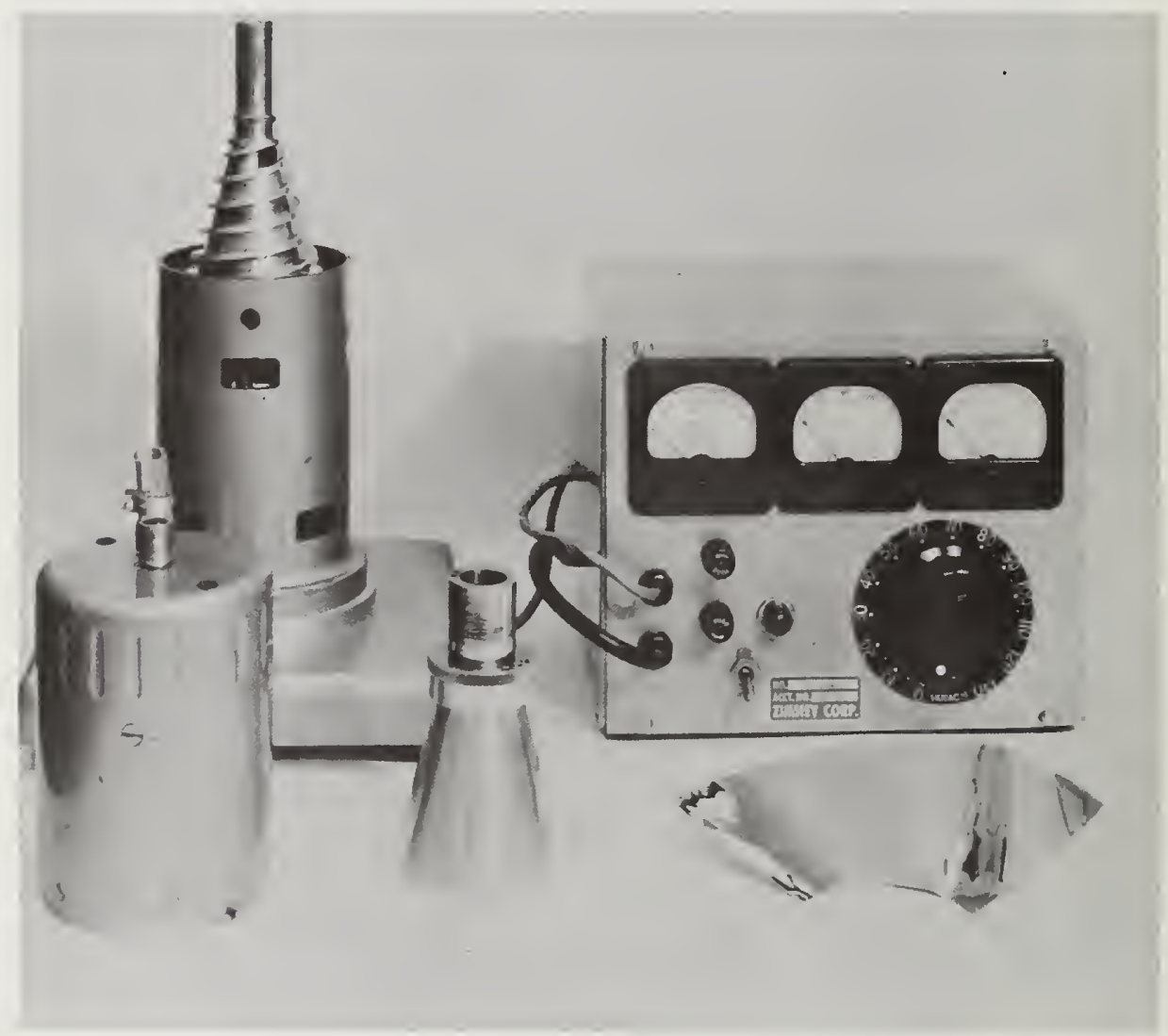

Figure 2. The Goetz Spectrometer.

Collection surfaces have included flour [16], absorbent paper [17], coated screens sensitized paper nonporous surfaces [20], and liquids [21]. The particles themselves are sometimes dyed [22], and sometimes frozen and sized by microscope [23] or by sedimentation [24]. Dissolved tracers are sometimes used, and the size reconstructed from the particle deposit [25]. For further information on this method see reference 4 . 


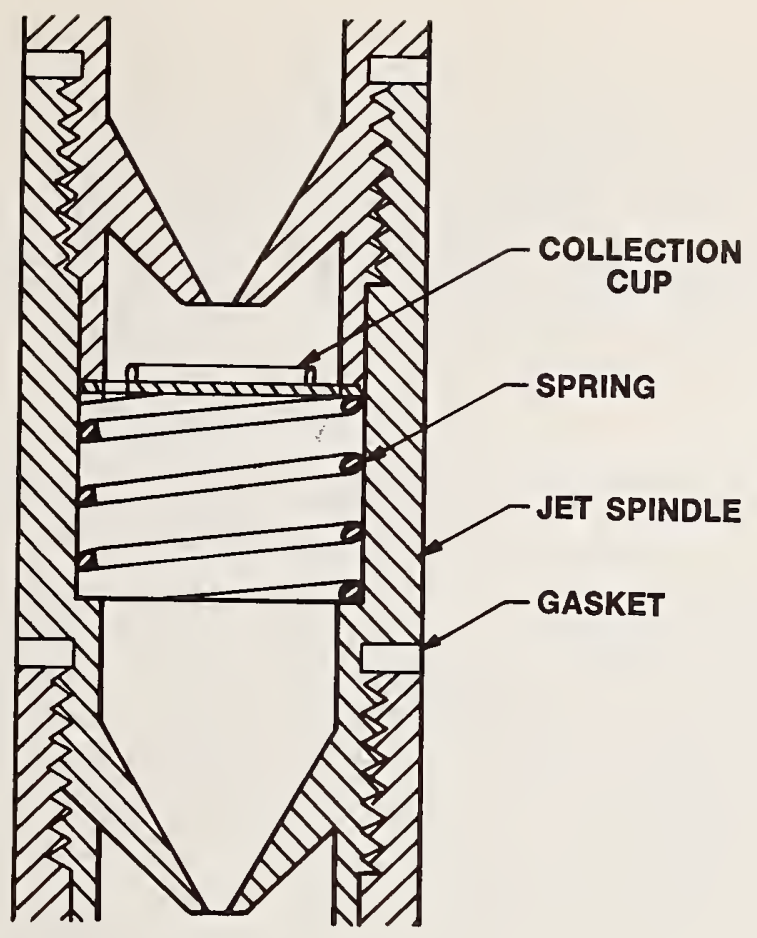

Figure 3. The Brink Impactor.

\subsection{Momentum Transfer}

This method is useful for volatile droplets of large size. Three types of methods have been used to measure size from momentum measurements. The size of craters in magnesium oxide coated surfaces was used by May [26]. The magnitude of the impact of a drop striking a microphone was used by Cooper [27], and the vibration of a piezoelectric crystal was used by Chuan [28]. A commercial version of this instrument manufactured by Celesco, is described later today in the paper by R. Chuan.

\subsection{Pulse Counting}

This method was reported by Pye [29], who used it for sizing oil droplets in fuel burners. The method is not considered applicable for spray can analysis. For more information, see reference 4.

\subsection{Hot-Wire Anemometry}

The output voltage fluctuations of a hot wire anemometer are a function of both the cooling of the wire by the air stream, and the evaporation of the droplets that impact upon the wire. The use of signal filters enable these effects to be separated, and size to be determined [7]. 


\subsection{Electrical Mobility Methods}

These methods determine the charge-weight relationship of the droplets. Mobility analyzers precipitate particles in an electric field of varying voltage, and measure the current due to the charges on the particle surfaces. The various instruments are discussed in references 4 and 7. Commercial instruments include those produced by ThermoSystems [7].

\subsection{Optical Methods} included:

Optical methods that have been used to measure droplet size have
a. Tynda11 Spectra
b. Single Particle Scattering
c. Mutti-Particle Scattering
d. Laser Scattering
e. Laser Holography
f. Laser-Radar
g. Laser-Interferometry

When a light beam passes through a monodispersed aerosol which is viewed at an angle to the incident light against a dark background, the presence of particles is observed by the occurance of Tyndall spectra due to the scattered light. The $0 w 1$ is an instrument that uses this principle to measure size [30]. With this device, the spectral colors are observed using a rotating telescope. With white light, size can be deduced from the number of red bands that appear in the spectrum.

Light scattering from single particles has been very widely used, and over the last 20 years, many variations in particle counting instruments have been developed. These are discussed in detail in references 4 and 5 .

Very recently, Gravatt [31] at NBS developed a light scattering counter for the EPA in which a simultaneous measurement of the light intensity at two small angles was performed. The ratio of these intensities was then related to size.

Use of the intensity ratio method at two angles is also made with multi-scattering particle systems. With the Bryce-Phoenix light scattering photometer, this technique, termed the polarization ratio method, is applied.

Other photometer systems using multiple particle scattering have been built and tested by Ranade [32] and Cooke and Kerker [33]. These systems were designed to measure the kinetic behavior of aerosol particles in flowing gas streams. 
The above light scattering devices use conventional optics, but other systems have been developed that measure the scattering from particles in a laser beam. Schleusener [34] developed a gas laser for sizing particles; Harris and Morse [35] developed a laser system for measuring fog droplet sizes. A recent device in this class was developed by Gucker et az. [36] and is the subject of the paper by Parmenter in this symposium. Knollenberg also later discusses laser scattering devices developed at Particle Measuring Systems Inc. in Boulder, Colorado.

Laser holographic methods have also found application in sizing droplet particles. Horn et az. [37] described a spray droplet analyzer, and offers a laser holographic service through Laser Holography Inc. This afternoon, Hotham describes the use of this system for sizing sprays from deodorant, hairspray, and pesticide spray cans. Optronics International offers a similar service, as do Laser Photographic Laboratories in Chicago. Hathaway [38] recently published data taken by this latter system on deodorant spray cans, and showed the effect of evaporation on the size distribution at distances of 7", 6", and 12" from the nozzle. The use of laser holography for sizing water drops was explored by Stigliani [39], who developed a forward scatter system at the Illinois Water Survey.

For meteorological studies, laser-radar methods have found application. Herman et az. [40] have reported on a system for measuring atmospheric aerosols, and Pasqualucci [41] has described the use of Doppler radar systems for rainfall measurement. Laser heterodyne spectroscopy, which measures a distribution of Doppler shifts, has been used by Gollub et az. [42] to measure the size of falling water droplets in a diffusion cloud chamber. The principle of this system and some size data are reported by Chabay in this symposium. The Doppler shift principle is also described today by Yanta, who has used it to measure size distributions of particles accelerated through a nozzle. In this work, the particle velocities were determined using the scattered light spectrum from the particles passing through an interference pattern.

\section{COMPARISON OF RESULTS FROM DIFFERENT DEVICES}

To give some estimate of the problems associated with droplet sizing, a comparative study on stearic acid aerosols was performed at IIT Research Institute. Some of the results are given in figure 4.

For this comparison, the methods using Tyndall spectra, single particle scattering, electrical mobility, particle collection, and image reconstruction were employed. The data shows that a mean size of $0.9 \mu \mathrm{m}$ was recorded by the Owl from Tyndall spectral measurements. This agreed fairly well with both the value of 0.84 um obtained by the collection, microscope measurement and particle reconstruction method, and the value of $0.8 \mathrm{\mu m}$ recorded by the mobility analyzer. The optical particle counters showed greater variability, which was mainly attributed to a 


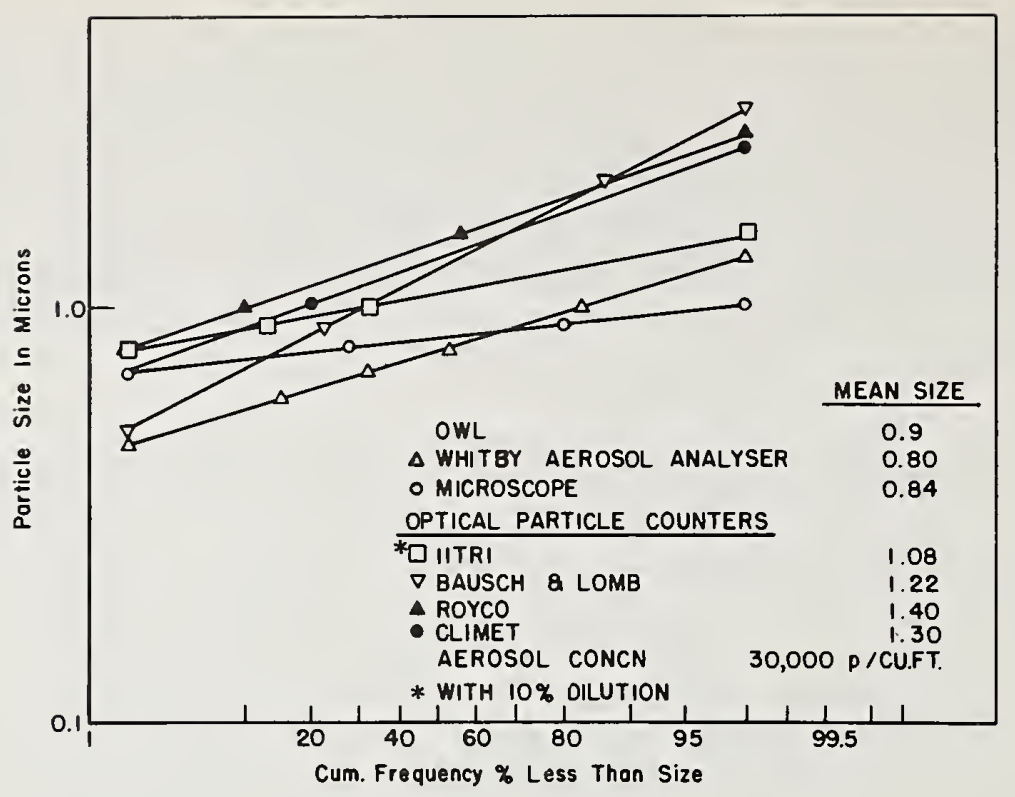

Figure 4. Comparative data taken on optical particle counters and the Whitby Aerosol Analyzer.

coincidence problem. With 30,000 particles/cubic feet, the Bausch and Lomb, Climet and Royco counters gave mean sizes rarging from 1.22-1.4 $\mu \mathrm{m}$. In contrast, the IITRI counter, operating with a 10:1 dilution system, gave a value of $1.08 \mu \mathrm{m}$.

In comparing these results, some correlation with the response time of the sizing methods should be reported. For example, the Owl was situated on the exit of the aerosol generator, and measured the aerosol at time $=0$. In contrast, the optical particle counters, the Whitby Aerosol Analyzer, and the electrostatic precipitator were connected to an $800 \mathrm{cu} \mathrm{ft}$ aerosol chamber, and measured the aerosol several minutes after generation. Allowing 5 minutes for homogenization of the aerosol after the completion of aerosol generation, al1 the instruments began their sampling period, at a time, $t,=5 \mathrm{~min}$. The optical particle counters then recorded the size distribution during a 1 minute sampling period, the Whitby Aerosol Analyzer recorded the size distribution during a 4 minute sampling period, and the electrostatic precipitator collected aerosol over a 20 minute sampling period.

It is important to note that the collection and the mobility analyzer results at $t=25$ and $t=9$ minutes, respectively, both indicated lower mean values than the Owl at time $=0$. As the standard deviation of the distribution by microscope was less than 1.2 , the aerosol can be termed monodispersed, and the Owl data should be reliable. This suggests that the analyses at time $=9$ min and time $=25$ min shows indication of slight size contraction of the aerosol. Though not considered an evaporative aerosol, stearic acid will undergo slight 
size changes of this kind. In contrast, evaporative aerosols, such as solvent droplets, would have shown wide differences, depending on the method, and time factor.

Though very brief, this data illustrates the point that droplet sizing data will be a function of the sizing method employed, the instrument used, the sampling time, and the characteristics of the aerosol. In consequence, in recommending a method for aerosol spray can analysis, the FDA, NIOSH, and EPA should consider this fact critically before proposing any specified technique to manufacturers and users of consumer products.

\section{POSSIBLE METHODS FOR SPRAY CAN ANALYSIS}

From the number of methods described in section 2, a few can be selected for use in spray can analysis. In general, the factors that have been taken into account in the selection of the following methods have been (1) size range of application, (2) speed of analysis of replication, and (3) cost of the method.

For consumer products, a size range of 1-300 $\mu \mathrm{m}$ is considered wide enough to cover most spray can droplet size distributions. For particles of this size and of high volatility, a maximum imaging and sensing time of 2 seconds is desired.

On this basis, laser holographic methods are considered the best for most applications. The method is fast, requires no aerosol confinement, and functions independent of the droplet composition. When coupled with image analys is equipment, the method is even better. However, the cost of such a total system is estimated to be $\$ 60,000$. By using a manual image analys is method, the capital cost can be reduced to $\$ 25,000$. With light scattering equipment, the analys is is again fast, but the aerosol does have to be confined, and the signal is dependent on the refractive index of the droplet. Further, to measure the size distribution, the aerosol must be dilute. A light scattering system capable of sizing aerosol sprays is estimated to cost around $\$ 10,000$. If low capital cost investment is a factor, then methods such as photographic imaging and impaction can be considered. With photographic imaging, the camera is the only investment if sizing is manually performed on the photograph. With impactors, sizing can be performed by a reconstruction of the droplet size from the size of the deposit on each stage, providing that the concentration of the solute is known. Alternatively, sensitized paper can be used as the deposition surface with an impactor of the Lundgren type. With this method, sizing would be manually performed by measuring the spots on the paper. For these low capital cost methods, the collection time is fast, but the analytical time is long and slow. Such devices would have a capital cost on the order of $\$ 1,000$. 


\section{CONCLUSIONS}

This brief review highlights the more important methods of sizing droplets, sprays, and mists. It specifically reviews the methods of application to aerosol spray can analysis, and recommends some techniques that are most suited to this purpose.

Of these, the most technically attractive is laser holographic imaging, but this involves a major capital cost investment. Light scattering systems are less expensive, but are also less technically attractive. Low capital cost methods that are useful include photographic imaging and impaction, but these are analytically slow and have high labor costs.

In selecting a method for the analys is of spray from aerosol cans, the capital cost-labor cost factor has to be resolved. If capital cost is no problem, then laser holographic imaging is considered the best choice.

\section{REFERENCES}

[ 1] Marchesani, V. J. et a.., J.A.P.C.A., 20 (1), 19, 1970.

[ 2] Harris, W. S., Arch. Int. Med., 131, 162, Jan. 1973.

[ 3] Environmental News, Wed., April 17, 1974.

[ 4] Davis, R., "Review of Methods for Sizing Droplets, Sprays, and Mists," IITRI Literature Review to be published.

[ 5] Davies, R., Proc. Internat. Conf. Particle Technology, IITRI, Chicago, August, 1973.

[6] Davies, R., American Laboratory, Part I, Dec. 1973.

[ 7] Davies, R., ibid., Part II, Jan. 1974.

[ 8] Davies, R., ibid., Part III, Feb. 1974.

[ 9] Mueller, E. and Sims, A., U.S. Army Electron. Command Report No ECOM-0032F (AD 645218).

[10] Meline, H., Test Eng., Sept. 1968.

[11] Webster, J.M., J. Photogr. Sci., 19, 38, 1971.

[12] Knollenberg, R. G., J. App. Met., 9, 86, 1970.

[13] Cannon, T. W., J. App. Meterology, 9, 104, 1970.

[14] Stockham, J. et a.., J. Physics E. (Sci. Inst.), 4, 557, 1971. 
[15] Spraying Systems Inc., N. Avenue, Wheaton, I17.

[16] Bentley, W., Mon. Weather Review, U.S.W.B., 32, 450, 1904.

[17] Defant, A., K Acad. Wiss. Math. Naturwiss., 114, 584, 1905.

[18] Mt. Washington Obs. Res. Report, Jan. 1951.

[19] Farlow, N. H., J. Col1. Sci., 11, 184, 1956.

[20] May, K. R., J. Sci. Instrum., 22, 187, 1945.

[21] Delavan Corporation Literature, West Des Moines, Iowa.

[22] Vooren, P. H. and Meyer, P. B., Am. Ind. Hyg. J., 134, Feb. 1971.

[23] Schbnauer, G., Staub, 27 (9), 7, 1967.

[24] Tanasawa, Y. and Hiroyasu, H., Tech. Rep., Tohuku Univ., 27, 67, 1962.

[25] Preining, 0. et al., J. Col1. Int. Sci., 23, 458, 1967.

[26] May, K. R., J. Sci. Inst., 27, 128, 1950.

[27] Cooper, B. F., Australian J. App. Sci., 2, 43, 1951.

[28] Chuan R. L., Aerosol Sci., I, 111, 1971.

[29] Pye, J. W., J. Inst. Fuel, 253, May 1971.

[30] Green, H. L. and Lane, W. R., Particulate Clouds, Van Nostrand, 1964.

[31] Gravatt, C. C., J.A.P.C.A., 28, 12, Dec. 1973.

[32] Ranade, M. B., Ph. D. Thesis, Illinois Inst. Technology, March 1974.

[33] Cooke, D. D. and Kerker, M., J. Coll. and Int. Sci., 42 (3), 535 , 1973.

[34] Schleusener, S. A., Pow. Tech., 2, 364, 1968.

[35] Harris, F. S. and Morse, F. L., J. Opt. Soc., 59 (11), 1518, 1969.

[36] Gucker, F. T. et al., Aerosol Science, 4, 389, 1973.

[37] Horn, J. et aZ., D.D.C. TISIA-2 Report 20 (AD 459 583), U.S. Dept. Commerce. 
[38] Hathaway, Aerosol Age, 20, Sept. 1973.

[39] Stigliani, D. J. et al., J. Opt. Soc. America, 60 (8), 1059, 1970.

[40] Herman, B. M. et al., J. Atmos. Sci., 28, 763, 1971.

[41] Pasqualucci, F., Proc. 15th Conf. Radar Meteorology, Champaign, I11., Oct. 1972. 


\title{
LIGHT SCATTERING BY SINGLE AEROSOL PARTICLES
}

\author{
Milton Kerker \\ Clarkson College of Technology \\ Potsdam, New York 13676
}

\section{ABSTRACT}

Although angular light scattering is a sensitive measure of particle size, sensitivity is rapidly lost when a mixture of particulates covers a range of particle sizes. However, instrumentation for studying scattering by single particles can overcome this limitation. This will be illustrated with reference to scattering by single fibers and spheres.

Key words: aerosol fibers; light scattering; particle size measurements; particulates; refractive index.

I shall touch upon three aspects of the art of particle size analysis by light scattering. These are (i) difficulties associated with the inversion of light scattered by dispersions, ( $i i)$ our experience with scattering by single particles, and ( $i i i)$ some recent calculations of the response curves for commercial particle counters. Background material may be found in reference 1 .

\section{THE PROBLEM OF INVERSION}

Light scattering by particles which are comparable in size to the magnitude of the wavelength of light is strongly dependent upon particle size, and it is for this reason that light scattering can provide a sensitive technique for particle size analysis. However, for a dispersion, the light signals are averaged over the particle size distribution. This has the effect of washing out the information and makes inversion of the light scattering-data to obtain the particle size distribution increasingly difficult as the distribution broadens.

This effect is illustrated in figure 1 , which is based upon calculations for refractive index $m=1.43$. The ordinate $\rho$ represents the ratio of the intensity of the parallel polarized component of the 


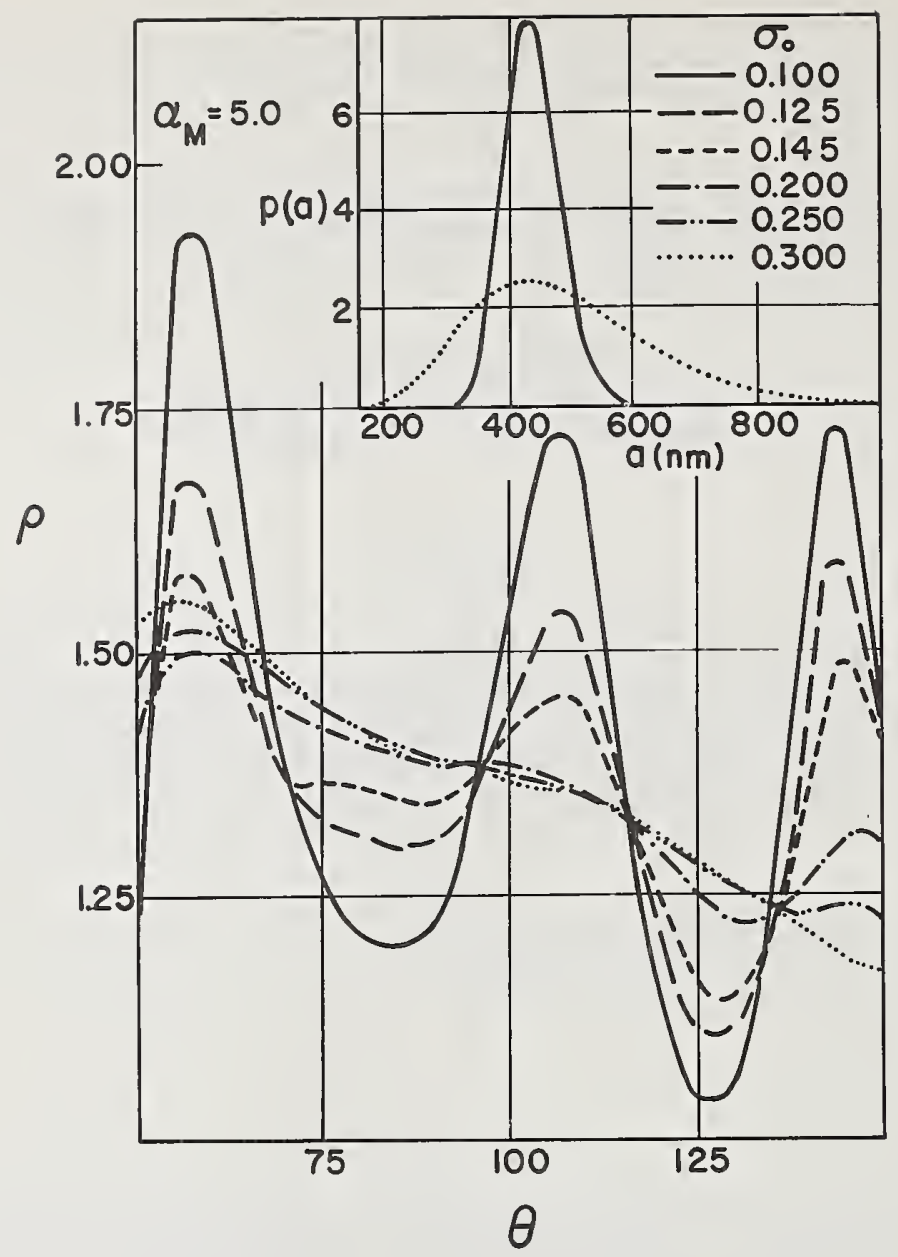

Figure 1. Polarization ratio $\rho$ plotted against scattering angle $\theta$ for $\alpha M=5.0$ and $\sigma_{0}=0.100,0.125,0.145,0.200$, 0.250 , and 0.300 . Inset shows distribution curves for $\sigma_{0}=0.100$ and 0.300 when $\lambda=0.546 \mathrm{~m}$.

scattered light to that of the vertical component. This is plotted against the scattering angle for the size distributions illustrated in the inset. These are logarithmic distributions with a modal optical size $\alpha=5$ (ratio of particle circumference to wavelength) and breadth parameters $\sigma_{0}=0.100,0,125,0.145,0.200,0.250,0.300$. (See ref. 1, p. 361).

For $\sigma_{0}=0.100$, the curve shows the typical oscillations characteristic of a narrow distribution. However, this structured character is obliterated as the distribution becomes broader. It is the structure in these curves, acting as a kind of fingerprint, that permits the precise 
determination of the size distributions from the light-scattering data. As the distribution becomes increasingly broader, it becomes increasingly difficult to obtain a unique solution. This is the dilemma. Narrowly dispersed colloidal suspensions are highly contrived laboratory preparations. Most systems encountered both in the laboratory and in nature are too polydisperse for particle size analysis by light scattering or by any other technique which depends upon inversion of such results. In this size range, $\sigma_{0}=0.20$ seems to be an upper limit.

On the other hand, quite accurate results can be obtained for a sufficiently narrow distribution. This is illustrated in figure 2,

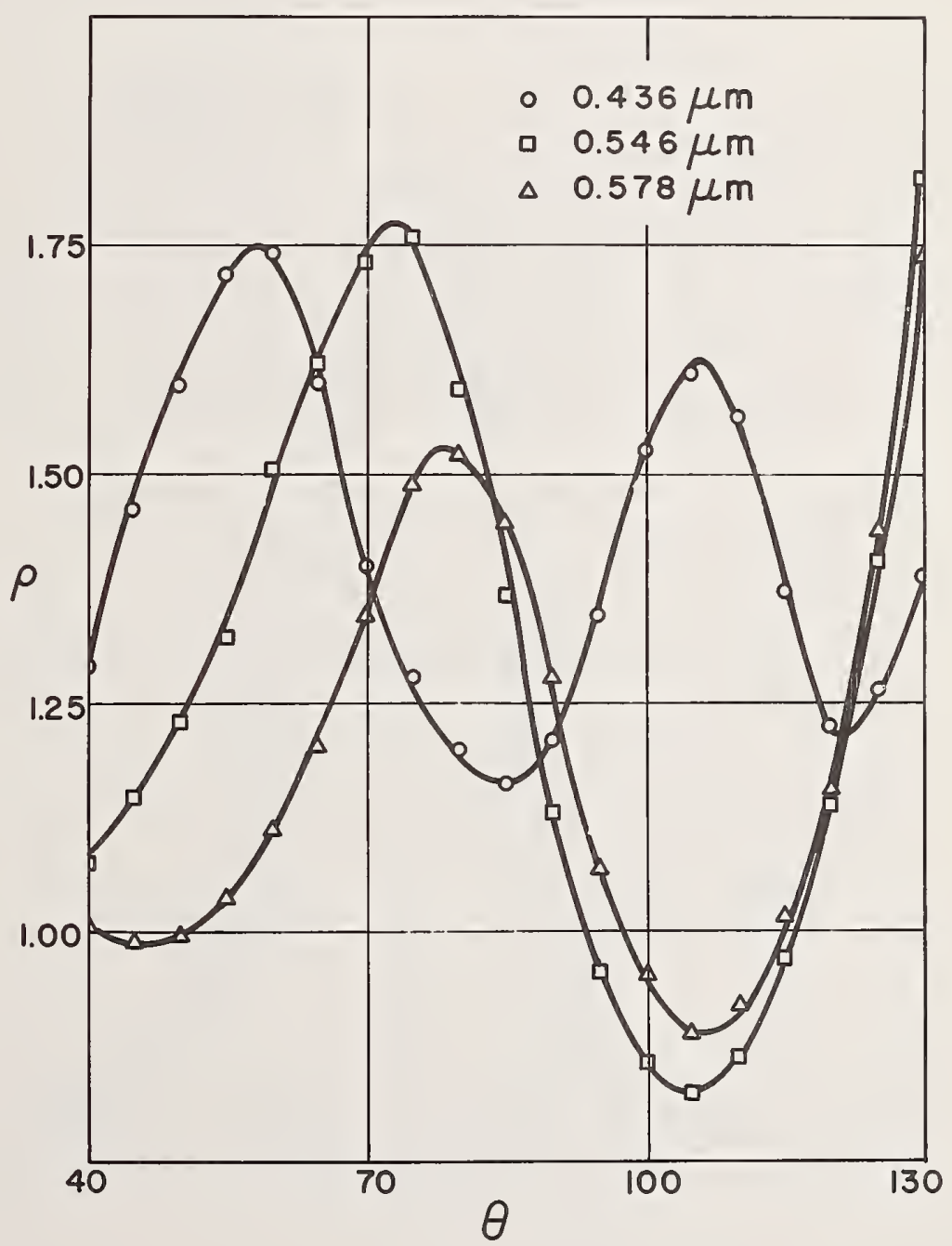

Figure 2. Experimental polarization ratio $\rho$ versus scattering angle $\theta$ obtained at three wavelengths, $\lambda=0.436 \mu \mathrm{m}, 0.546 \mu \mathrm{m}$, and $0.578 \mu \mathrm{m}$ for $\mathrm{a}$ dibutylphthatate aerosol. 
which gives experimental results for a dibutylphthalate aerosol obtained at three wavelengths, $\lambda=0.436,0.546$, and $0.578 \mu \mathrm{m}$. Because the size parameter $\alpha$ depends on wavelength, the light-scattering curves for the same system differ in the indicated manner at the different wavelengths. Yet, internal consistency requires that inversion of these data yield the same distribution of particle radii. The corresponding particle size distributions plotted in figure 3 show that whereas the scattering curves are quite different, the distributions calculated from these two sets of data are in excellent agreement.

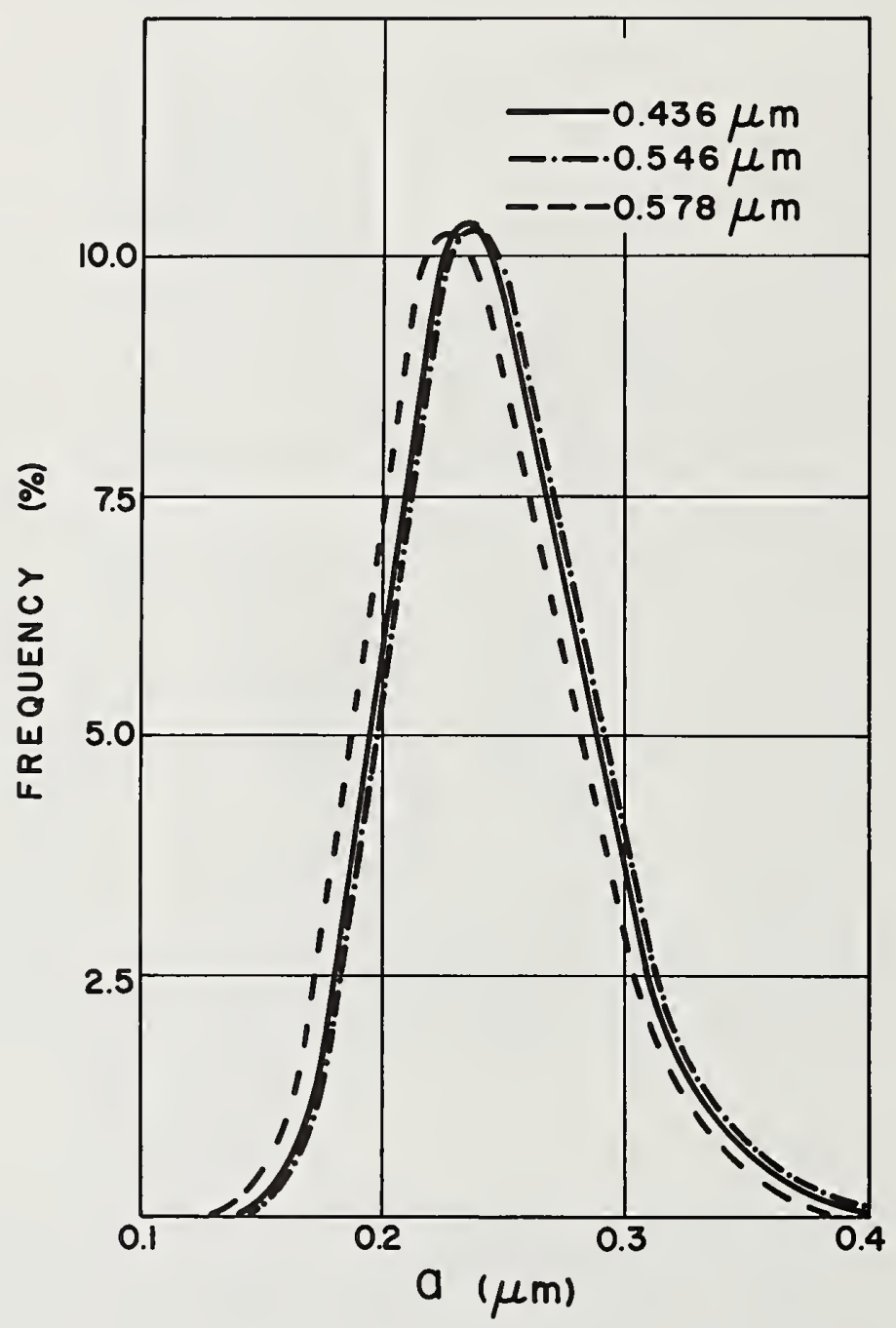

Figure 3. Particle size distributions corresponding to the data in figure 2. 


\section{RESULTS FOR SINGLE PARTICLES}

The potential of the light-scattering method can be illustrated by some results obtained with single fibers obtained a number of years ago $[1]$.

The physics of scattering by long circular cylinders is quite analogous to that by spheres, and so the potentialities can be appreciated if we consider the case illustrated in figure 4 . The ordinate is the polarization ratio and as in figure 2 this is plotted against the scattering angle. The circles show the experimental results for a particular silica fiber with unpolarized light of $546 \mathrm{~nm}$. These are compared with theoretical calculations shown as smooth curves for six combinations of the refractive index and the size parameter.

The results are striking. The best fit between the experiments and theory is for $m=1.46$ and $\alpha=4.00$ as depicted in the lower center panel of figure 4. When $\alpha=3.98$ or 4.02 , the theoretical results differ from the experiments as shown. Similarly, there are differences between theory and experiment for $\alpha=4.00$, but with $m=1.45$ or 1.47 . In effect, as these diagrams show, this represents a determination of the size of this fiber to an accuracy of about 0.25 percent. This is remarkably accurate. For example, size determination in this range by electron microscopy is hardly better than about 5 percent.
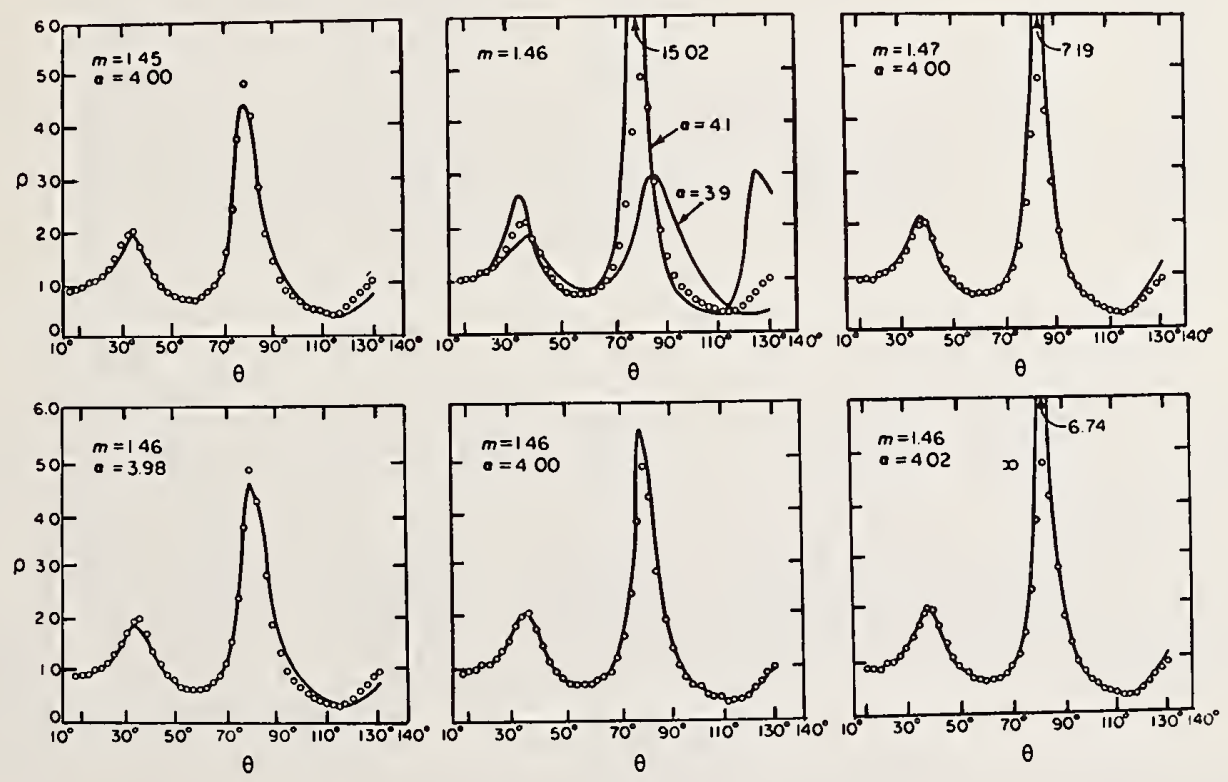

Figure 4. Polarization ratio $\rho$ plotted against scattering angle $\theta$ for a cylinder illuminated at perpendicular incidence. Comparison of experimental results (circles) and theory (smooth curves) for a silica fiber at $\lambda=$ $0.546 \mu \mathrm{m}$. 
With the availability of a commercial single particle light scattering photometer*, it has become possible to make measurements of dispersions, a single particle at a time. We have determined the particle size distribution of a sample of polystyrene latex as obtained particle by particle using this instrument.

The size distribution made up of 331 single particle runs is shown in figure 5 as a histogram of percent frequency versus size parameter $\alpha$. The maximum frequency is at $\alpha=4.79$ with 90 percent of the particles between 4.58 and 4.90 . The remaining particles are grouped discretely in the ranges $a=3.0$ to 3.3 and $a=3.72$ to 4.32 .

The two smaller populations have not been reported before. It is not surprising that they would not be detected by conventional light scattering. For example, we have calculated the light-scattering rad-

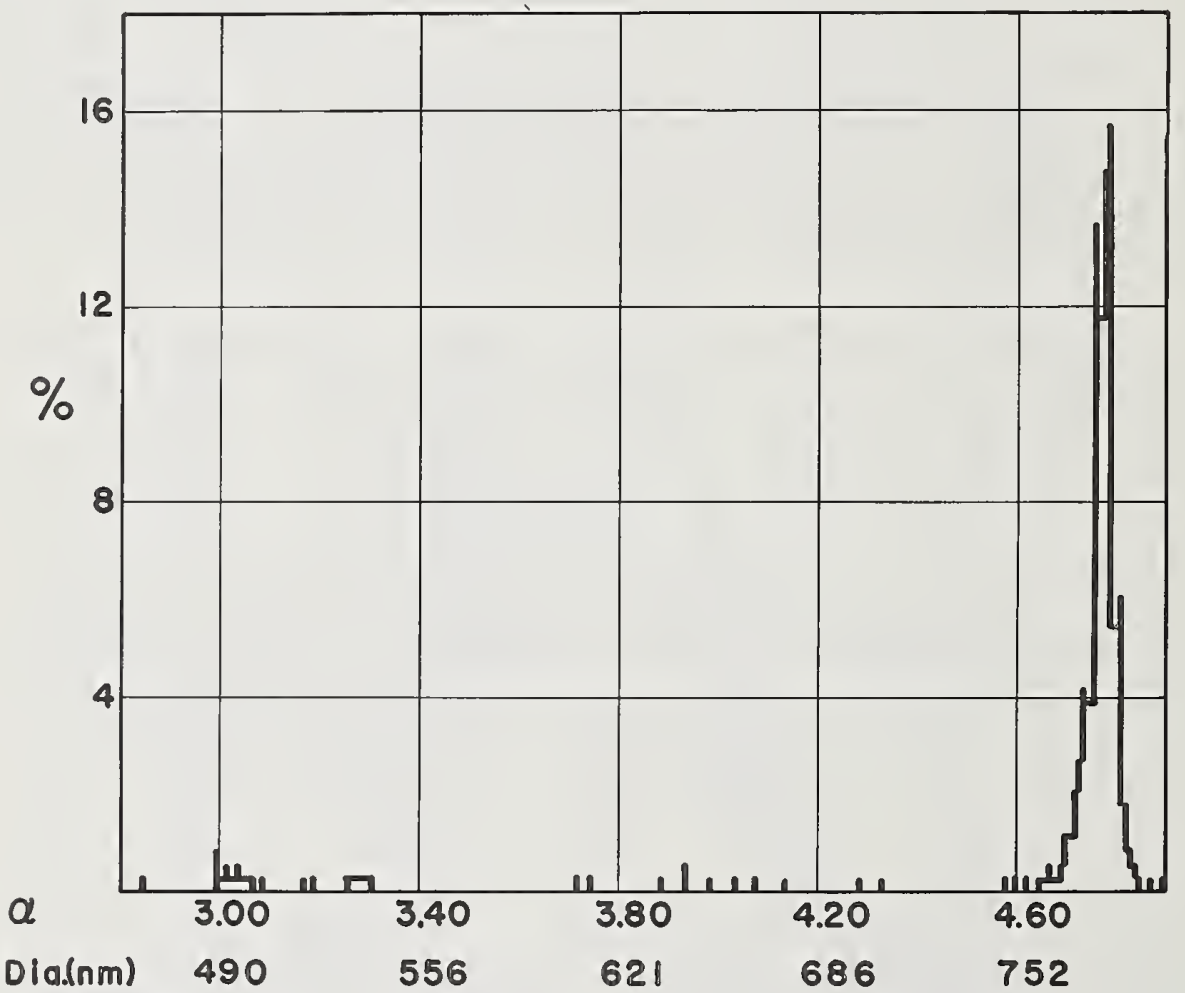

Figure 5. Histogram of particle sizes of a polystyrene latex obtained by light scattering from 331 single particles.

*Differential II Light Scattering Photometer manufactured by Science Spectrum, P. 0. Box 303, Santa Barbara, Calif. 
iances that would be obtained for the distribution depicted in figure 5 and then have inverted these results on the assumption that the system was a logarithmic distribution. This gave a modal size $\alpha_{M}=4.75$ instead of 4.90 and $\sigma_{0}=0.02$. Thus, the smaller class of particles affects the estimate only slightly, and if there were not some a priori information concerning them, there would be no reason, on the bas is of the light-scattering data, to suspect their presence. Accordingly, light scattering from the assembly of particles cannot be used to detect the presence of these smaller particles.

We also determined the size distribution of this latex in the conventional manner using the liquid suspension. The results for the average diameter are compared in table 1 with determinations in other laboratories.

Table 1. Comparison of average (or modal) diameters of Dow Polystyrene Latex.

Method

Diameter

$\mathrm{nm}$

Particle by particle light scattering (this work)

a) Major population group

b) All particles

784

764

Hydrosol light scattering (this work)

797

Hydrosol light scattering, Kratohvil and Wallace

773

Electron microscopy, Dow Chemical Company

796

Electron microscopy, Robbins and Jizmagian

777

Electron microscopy, Davidson et al.

765

The differences are probably within the experimental errors of the techniques. The principal advantage of the single particle techniques (both light scattering and electron microscopy) is that no a priori assumptions are required regarding the form of the distribution function. Indeed, the negative skew of the main population group in figure 5 and the presence of the two classes of smaller particles could not be predicted nor can their presence be confirmed using conventional lightscattering techniques.

The failure heretofore to observe the smaller particles calls for further investigation, using both light scattering and electron microscopy. Also, much work remains to be done to assure the lack of bias in the sampling procedure for the single particle light scattering; otherwise, the question of the skewness of the distribution cannot be resolved definitively. In any case, the prospect for applying light scattering to size distributions broader than any heretofore amenable to light-scattering analysis appears to be a reasonable prospect. 


\section{RESPONSE CALCULATIONS FOR LIGHT-SCATTERING}

PARTICLE COUNTERS

We have recently completed response calculations for five commercial light-scattering aerosol particle counters which take into account the emissive power of the light source, the spectral sensitivity of the phototube, and the specific geometrical factors for each instrument. Earlier calculations had been published for two of these instruments, but these earlier calculations had not considered each of these factors appropriately. The results will be published elsewhere [3]. Here we wish only to state that there is a strong dependence of the response upon both the real and the imaginary part of the refractive index and, for a given refractive index, a multivalued response in the submicrometer range for three of the five instruments. Certainly these results indicate that particle size determination with any of these instruments will be highly precarious whenever the refractive index of the particles is unknown. Furthermore, the hazards of venturing to extend the use of these instruments for particle size analysis of nonspherical particles are quite unpredictable.

\section{REFERENCES}

[1] Kerker, M., "The Scattering of Light and Other Electromagnetic Radiation," Academic Press, New York, N.Y., 1969.

[2] Cooke, D. D. and Kerker, M., J. Colloid Interface Sci. 42, 150 (1973).

[3] Cooke, D. D. and Kerker, M., Appl. Optics. Submitted for publication. 
NATIONAL BUREAU OF STANDARDS SPECIAL PUBLICATION 412

Aerasal Measurements, Praceedings af a Seminar an Aeras al Measurements

Held at NBS, Gaithersburg, MD, May 7, 1974 (Issued Octaber 1974).

\title{
LIGHT SCATTERING METHODS FOR THE CHARACTERIZATION OF PARTICULATE MATTER IN REAL TIME
}

\author{
C. C. Gravatt \\ National Bureau of Standards \\ Washington, D.C. 20234
}

\section{ABSTRACT}

This paper presents a brief overview of new light scattering methods for the rapid characterization of particulate matter in air. An instrument has been developed which determines the size distribution of particulate matter in air in essentially real time by a forward lobe light scattering method. The basic concept involves the simultaneous measurement of the intensity of light scattered by a single particle at two small scattering angles. The ratio of the two intensities is a direct measure of the size and is fairly independent of the index of refraction of the particle. Numerical solutions of the Mie equations for spheres have indicated that the sizing error by this method is not greater than $\pm 15 \%$ for the range of particle sizes from 0.2 to $4 \mu \mathrm{m}$ for essentially all possible indices of refraction. In addition, it appears feasible to extend the lower limit of size determination to $0.05 \mu \mathrm{m}$ by the measurement of a single forward lobe intensity. Also, a technique has been developed which permits some degree of chemical characterization of particles, and which has been employed in a smoke detector capable of distinguishing between fire-produced and nonfireproduced aerosols.

Key words: aerosol sizing; aerosol spectrometer; chemical characterization of particles; fire produced particles; laser light scattering by aerosols; particle size measurements; particulates refractive index; smoke detector.

\section{INTRODUCTION}

The National Bureau of Standards, as part of its Measures for Air Quality Program (MAQ), has been investigating rapid, light scattering methods for the characterization of particulate matter. These investigations have primarily dealt with particles in air with diameters in the respirable size range $(0.05 \mu \mathrm{m}$ to $5 \mu \mathrm{m})$ although the techniques described below can be modified to operate over other size ranges and in other media, such as water. A single particle light scattering instrument has been developed which is based on a forward-lobe-ratio technique and which covers the particle diameter range from $0.2 \mu \mathrm{m}$ to 4 
$\mu m$. Theoretical calculations for spheres have shown that the particle size error, due to variations in index of refraction, is approximately $\pm 15 \%$ for essentially all possible materials. This technique has been incorporated into a field compatible instrument constructed for and partially supported by the EPA, which will be part of the Regional. Air Pollution Study (RAPS) to get comparative data among various particle sizing instruments. In addition, a method has been developed which permits some degree of chemical characterization of particles, specifically, a determination of whether a particle is carbon like, metal like, or something else. This method has been incorporated into a smoke detector which can distinguish between fire-produced and nonfireproduced aerosols.

Light scattering methods provide a useful way of sizing particulate matter in air in essentially real time and without the necessity of collection of the sample. In addition, the size range of most interest for lung interaction, $0.05 \mu \mathrm{m}$ to $5 . \mu \mathrm{m}$, is relatively easily detected by light scattering if moderate power lasers are employed as sources. However, the scattered intensity is not only a function of the particle size but depends rather strongly on the particle index of refraction. As a result, in those instances where a wide and unknown range of indices of refraction may exist in the sample, the light scattering method may give erroneous size values. It is found that errors on the order of factors of 2 or greater are not uncommon when sizing particles which have indices of refraction differing from that of the calibration species [1]. It was first pointed out by Hodkinson [2] that the forward lobe region of the particle scattering spectra is least affected by the index of refraction and provides a convenient method of sizing particles to a moderately high degree of accuracy.

\section{PARTICLE SIZING}

An instrument has been developed which limits the error due to unknown particle index of refraction to approximately $\pm 15 \%$ for essentially all possible types of aerosols. The basic concept of this method involves the measurement of the intensity of light scattered by a single particle at two small scattering angles and the calculation of the ratio of these two intensities. The intensity ratio is directly related to the size of the particle and is essentially independent of the index of refraction of the particle. The intensity ratio method is also attractive since it eliminates or minimizes complications resulting from a number of experimental phenomena, such as source intensity variations and nonuniform particle velocities. Since this instrument and its theoretical evaluation has been discussed in several publications only a brief summary is given below $[1,3,4]$.

Figure 1 provides an introduction to the concepts used for both particle sizing and chemical characterization. This figure shows the scattered intensity, in arbitrary units, as a function of scattering angle, $0^{\circ}$ to $180^{\circ}$, for a particle diameter of $1.0 \mu \mathrm{m}$ and an incident wavelength, $\lambda$, of $514.5 \mathrm{~nm}$. The incident beam is assumed to be linearly polarized and the scattered intensity is measured in the plane of 


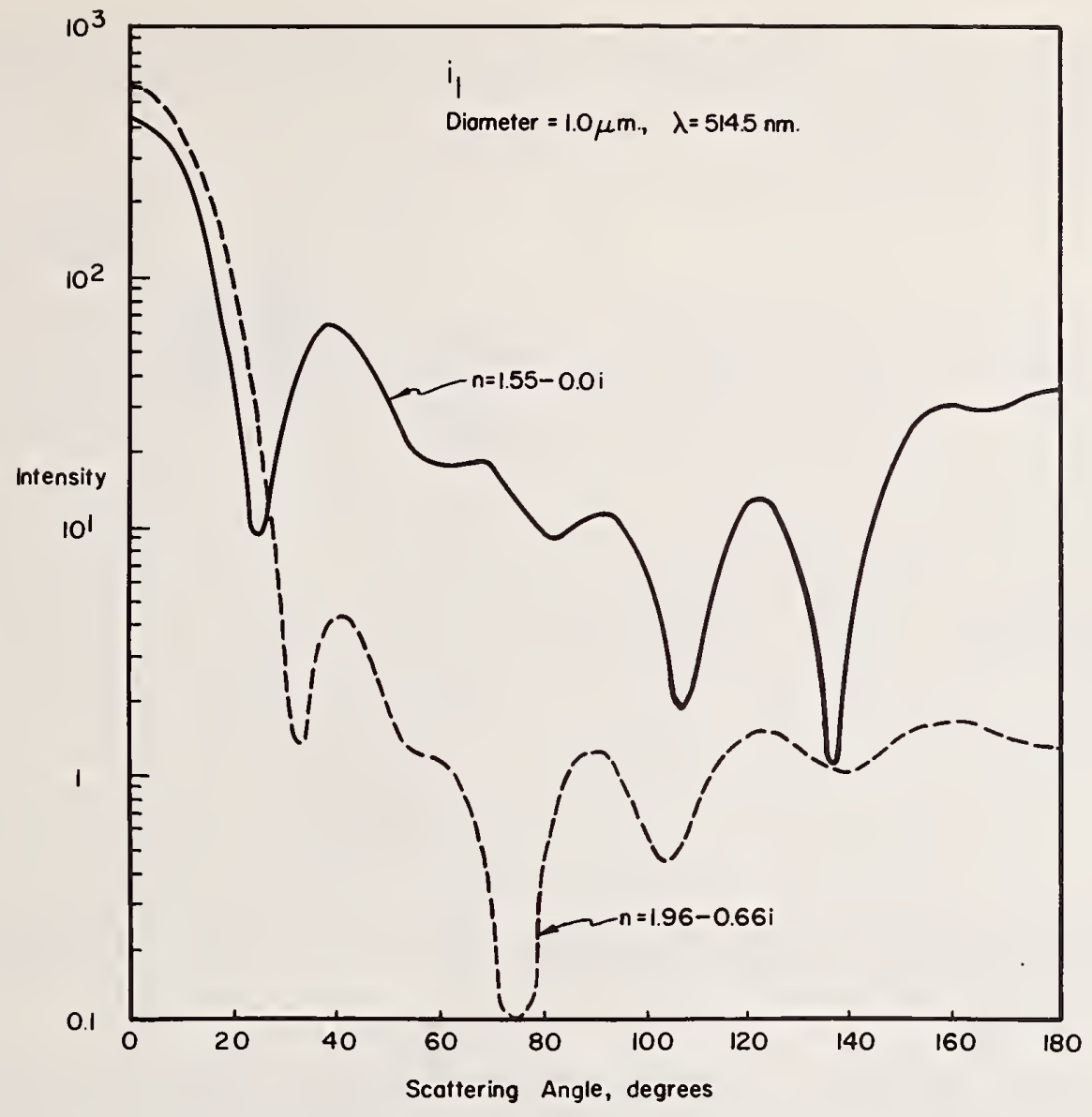

Figure 1. Scattered intensity as function of scattering angle in the plane of polarization. $n=$ index of refraction. $\lambda=$ wavelength of incident radiation.

polarization. The two particles are one with an index of refraction of $n=1.55-0.0 i$, representing a nonabsorbing material with an index similar to many earth dusts, and the second a carbon particle, with index of $1.96-0.66 \mathrm{i}$. It can be seen that the forward lobe for these two materials are very similar in shape even though the intensity differs by a factor of two or so. The similarity of shape of the forward lobe for widely varying indices of refraction is the phenomenon employed for particle sizing. Specifically, a ratio of two intensities in the forward lobe is measured. The angular ratio of $20^{\circ} / 10^{\circ}$ is effective over the particle range $0.1 \mu \mathrm{m}$ to $1.5 \mu \mathrm{m}$, the ratio $10^{\circ} / 5^{\circ}$ is effective over the range $0.2 \mu \mathrm{m}$ to $3.5 \mu \mathrm{m}$ and the ratio $5^{\circ} / 2.5^{\circ}$ is effective over the range $0.5 \mu \mathrm{m}$ to $10 \mu \mathrm{m}$ if the incident wavelength is approximately $500 \mathrm{~nm}$. Shorter and longer wavelengths decrease and increase these ranges respectively. Also, it can be seen that the region from 30 to $90^{\circ}$ differs by about one order of magnitude for these two materials. This effect is found to be generally true of absorbing and nonabsorbing materials and permits the partial chemical characterization of particles. 
Figure 2 is a block diagram of the optical and electrical sytem used in the original instrument. The EPA instrument differs slightly in ways which are given below.

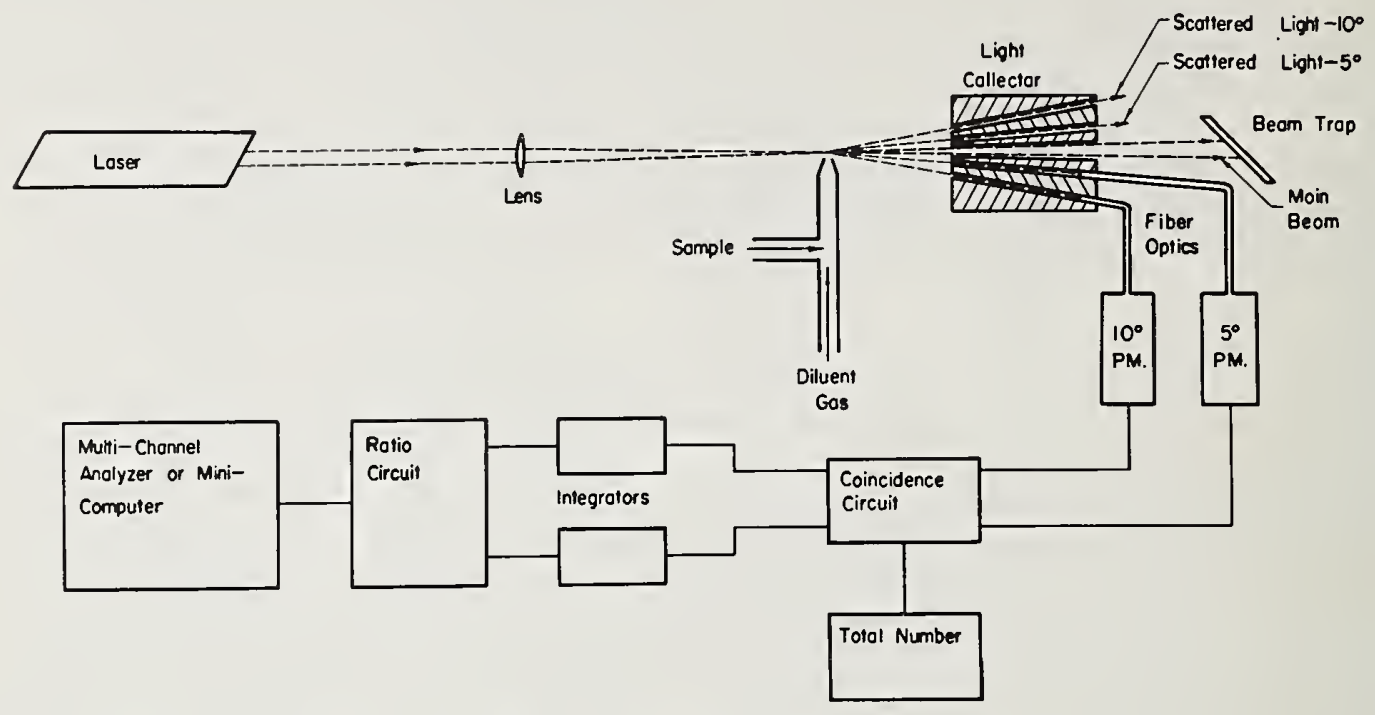

Figure 2. Block diagram of forward-lobe-ratio particle sizing instrument.

The light from a CW laser, either argon ion or helium-neon, is focused to a small point by a lens, and the sample blown through this point. A flow rate is chosen such that a particle takes approximately $10 \mu \mathrm{s}$ to pass through the beam. When a $2 \mathrm{~mW} \mathrm{He}-\mathrm{Ne}$ laser is used as the source, it is found that the smallest particle which can be sized effectively is approximately $0.4 \mu \mathrm{m}$ in diameter due to insufficient scattered intensity for smaller particles. However, when the source is an argon laser of $10 \mathrm{~mW}$ or higher power, particles can be sized to the limit of resolution of the ratio method, $0.2 \mu \mathrm{m}$ for the angles of $10^{\circ}$ and $5^{\circ}$. Smaller particles than this are "seen" and, therefore, counted by the system, but the ratio is relatively insensitive to particle sizes below $0.2 \mu \mathrm{m}$. The EPA instrument employs an air cooled $10 \mathrm{~mW}$ argon ion laser as source.

The light scattered by a particle is measured by the light collector consisting of three parts and operating as a fixed, two angle, annular iris detector. The innermost section has a hole in its center to allow the main beam to pass through and an outer conical shape at an angle of $5^{\circ}$ relative to the optical center. The second section has an inner conical shape of $5^{\circ}$ and an outer conical shape of $10^{\circ}$. The outer most section has an inner conical shape of $10^{\circ}$. Optical fibers are positioned in the rear of the collector system and these fibers are grouped in two bundles, one each for the scattering angles of $5^{\circ}$ and $10^{\circ}$. The output ends of the fiber optic bundles are placed close to the photocathodes of two photomultiplier tubes. When a particle passes 
through the light beam a pulse will be produced in the output of each photomultiplier. The height of these pulses is proportional to the scattered light intensity and the width is the time of flight of the particle through the beam. The optical system of the EPA instrument is similar except the fiber optics have been replaced by a series of lenses, mirrors, and baffles.

The active optical region is defined by the diameter of the laser beam, $0.14 \mathrm{~mm}$, and the diameter of the sample stream $0.1 \mathrm{~mm}$, and it has a volume of $1.5 \times 10^{-5} \mathrm{~cm}^{3}$. Therefore, concentrations as high as $2 \times$ $10^{4}$ particles $/ \mathrm{cm}^{3}$ can be measured without coincidence effects being larger than $2 \%$ of the total counts. At higher particle densities the sample stream can be diluted to reduce the concentration to an acceptable level and thus prevent coincidence losses caused by two or more particles being in the beam at the same time. The scattered light is detected by the two photomultipliers, and level detectors in a coincidence circuit are employed in each output channel to define that period of time during which a particle is "seen" by both detectors . During this time the scattered intensity at each angle is integrated in analog circuits (in the EPA instrument the peak intensity is detected). At the completion of the passage of the particle through the beam an output pulse is generated whose height is proportional to the ratio of the two integrated intensities. These pulses are counted either in a multichannel analyzer or a minicomputer to determine the particle size distribution.

It is important to note that by taking a ratio of the two scattered intensities, most experimental complications are eliminated. There is no uncertainty caused by particles taking different times to pass through the light beam, or by passing through different parts of the beam, or by variation in intensity of the laser. In addition, it is possible to design the logic and timing system so that in the event that two particles are in the beam at the same time, no data are taken.

The performance of this instrument has been tested on a number of particulate samples. The primary calibration has been performed using polystyrene latex sphere samples of accurately known diameter. In addition to the latex sphere samples, this system has been tested with a variety of materials having wide ranges of indices of refraction and nonspherical shapes. These samples include zinc cadmium sulfate (fluorescent), tungsten, iron oxide, carbon black, titanium dioxide, puff ball spores, kaolin, and silica. Since these materials have not been accurately characterized as to their size distribution by an independent method, it is impossible to reach quantitative conclusions, but from the reported data on mean size or size range it has been found that this ratio instrument is not making large errors due to index of refraction or shape. It is estimated that the total size error, assuming a calibration performed with polystyrene latex spheres, is no greater than $20 \%$. 
Numerical solutions of the Mie equations for spheres have been performed to evaluate theoretically the optimal parameters and the inherent error in the forward lobe ratio method $[4,5]$. These calculations were performed for essentially all possible indices of refraction that might be expected in ambient air and other special types of particulate samples such as fire-produced aerosols. Specifically, the real component of the index of refraction was investigated over the range 1.33 to 2.0 and the imaginary component investigated over the range 0.0 to 4.0 . This range of indices will cover most inorganic and organic materials, metal ores, earth dusts, carbons, and the metals. The results of these calculations have shown that the inherent error in the particle size, due to index of refraction variation in the sample, is no greater than $15 \%$ for the following combination of particle size ranges and angular ratios, assuming the particles are spherical. The angular ratio of $20^{\circ} / 10^{\circ}$ is effective over the particle range $0.1 \mu \mathrm{m}$ to $1.5 \mu \mathrm{m}$, the ratio of $10^{\circ} / 5^{\circ}$ over the range $0.2 \mu \mathrm{m}$ to $3 \mu \mathrm{m}$ and the ratio $5^{\circ} / 2.5^{\circ}$ over the range $0.5 \mu \mathrm{m}$ to $10 \mu \mathrm{m}$ if the incident wavelength is approximately $500 \mathrm{~nm}$. Shorter and longer wavelengths decrease and increase these ranges respectively. The effect of angular aperture was found to be smal1, and moderate apertures of approximately $0.5^{\circ}$ are advantageous in that they increase the scattered intensity and do not degrade the size sensitivity. Various ratios were investigated, and it was found that those with a value of 2 are as effective as any other ratio and lead to the most symetrical calibration curve.

Figure 3 shows the theoretical error envelope for the intensity ratio of $\mathrm{I}_{10} / \mathrm{I}_{5}$ for unpolarized incident radiation. Also shown is the response curve for particles with index of refraction of $1.50-0.0 i$. The error envelope incloses all response curves and can be used to determine the theoretical error of forward lobe ratio instruments. In general, the individual response curves are not monotonic functions, and therefore, in those situations in which the particle index of refraction is known, the forward lobe ratio technique may not be the most accurate method to employ. Also note that beyond a diameter of $3.0 \mu \mathrm{m}(\alpha=\Pi D / \lambda$ = 19) the ratio envelope increases. To prevent substantially higher errors due to this effect, an intensity cutoff is employed to prevent the recording of a ratio for particles larger than this cutoff.

We have investigated theoretically several ways of decreasing the size error. The most promising of these is to use a multiwavelength laser as a source, especially one that has two widely-spaced lines. For example, an argon-krypton laser, with blue, green, and red wavelengths, would have an error of approximately 3\% rather than the 15\% error for a single wavelength. It appears that it is important that the source contain two or more widely spaced lines rather than having a continuous wavelength distribution as in incandescent sources.

An extension of the theoretical studies described above $[4,5]$ has indicated that particles in the diameter range $0.05 \mu \mathrm{m}$ to $0.2 \mu \mathrm{m}$ which are below the resolution of the ratio method (although they can be detected by the system) can be sized by a single forward lobe intensity or by a difference of two forward lobe intensities. The theoretical 
Diameter $(\mu \mathrm{m})$ for $\lambda=514.5 \mathrm{~nm}$.

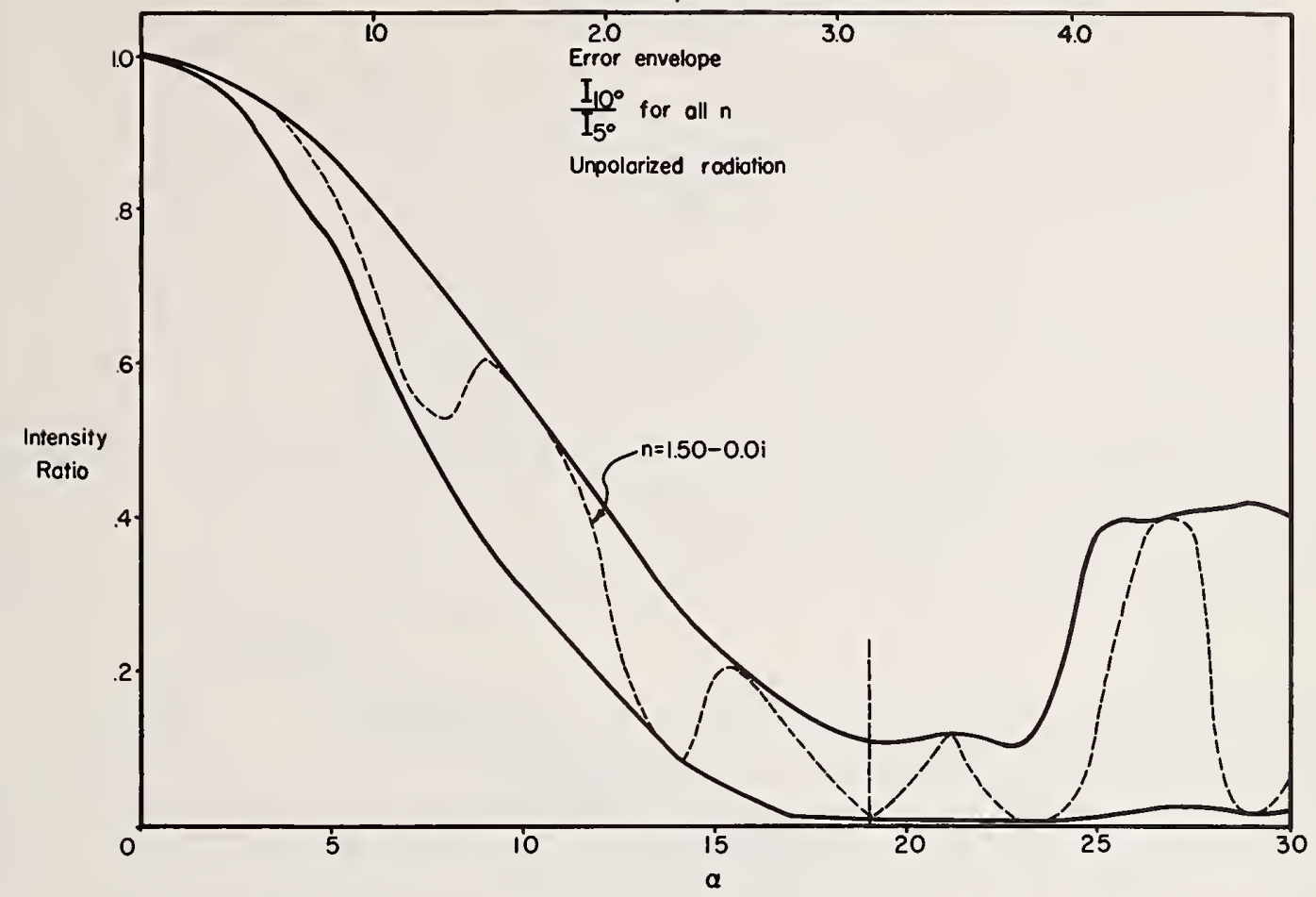

Figure 3. Error envelope for particle sizing instrument. Intensity ratio is for $I\left(10^{\circ}\right) / I\left(5^{\circ}\right)$, unpolarized incident beam and no detector aperture. $\alpha=\Pi($ diameter $) / \lambda$ cutoff by intensity at $\alpha=19$.

error of these techniques is approximately $25 \%$ for all possible indices of refraction. However, the use of a single intensity or a difference of intensities will produce experimental errors which are not of concern in the ratio method. One possible way of eliminating the majority of the experimental errors is to normalize the intensity data by the time of flight of the particle through the laser beam. The time of flight is relatively easily determined and serves as a measure of the main beam intensity incident on each particle.

\section{CHEMICAL CHARACTERIZATIONS}

In many situations some degree of chemical characterization of particulates would be advantageous, especially if it could be accomplished without collection and subsequent chemical analysis of a sample. As an outgrowth of the theoretical studies described above, which are concerned with a study of particle sizing methods, a technique was discovered which seems to offer a way of determining some information related to the chemical composition of each particle as it passes through the laser beam. The information that can be obtained is a function of the imaginary component of the particle's index of refraction. 
Figure 4 shows how chemical information can be deduced. The quantity I, represented as the integral over $40^{\circ}$ to $70^{\circ}$ of the scattered intensity in the plane of polarization is shown as a function of particle size for a series of absorbing and nonabsorbing materials. I can also be considered as the output of an intensity detector in the polarization plane with an aperture from $40^{\circ}$ to $70^{\circ}$. For particles smaller than $0.3 \mu \mathrm{m}$, it is found that $I$ is insensitive to chemical character but for larger particles the discrimination is quite good. The band represented by $k=0$ is the envelope region for all values of the real component of $n$ but with no absorption character.

A fairly complete analysis of this effect has shown that the angular region from $40^{\circ}$ to $70^{\circ}$ is the most effective region to employ for discrimination among materials [6].

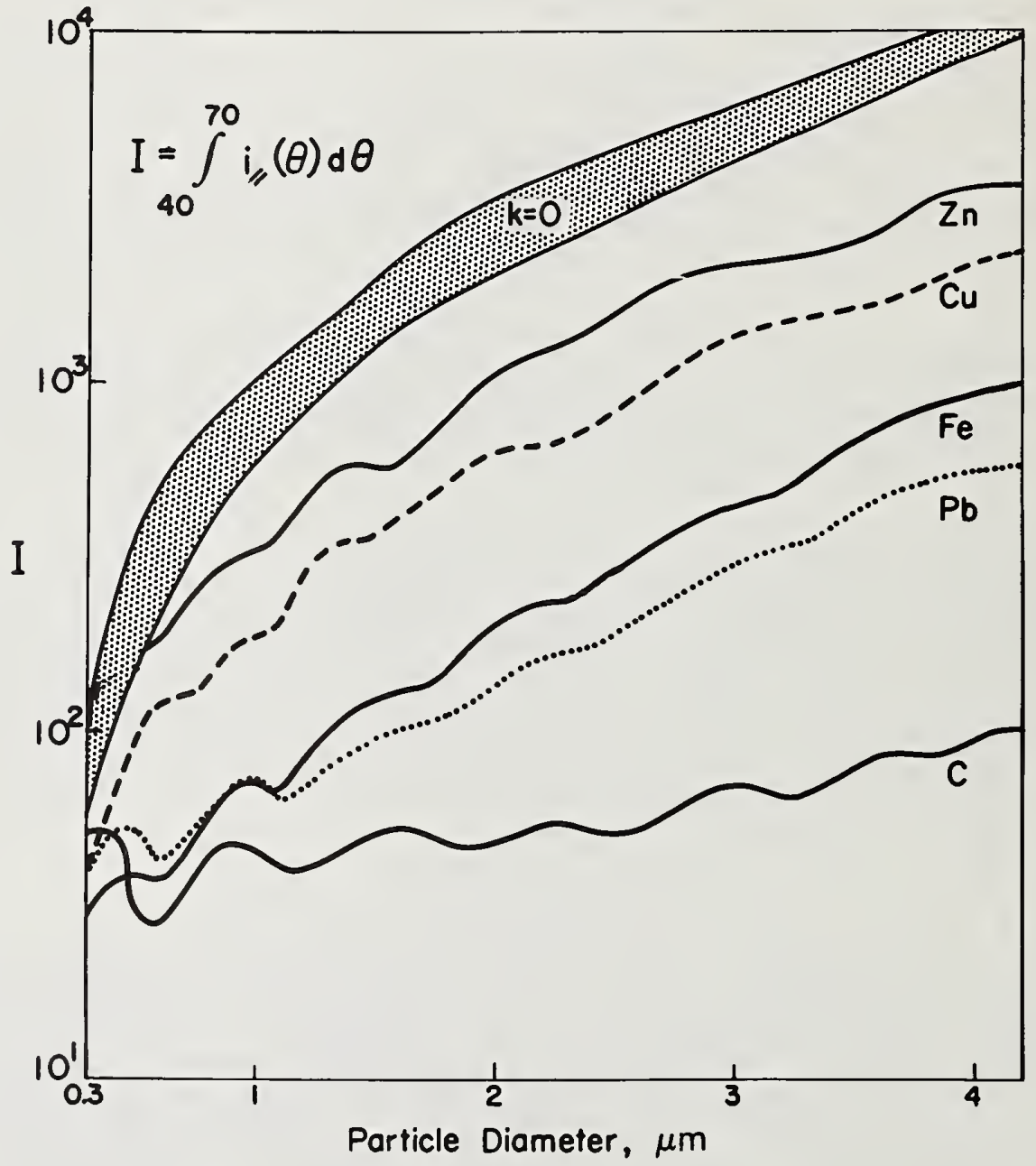

Figure 4. Detection of absorbing and nonabsorbing particles. $k=0$ region is for wide range of real components of index of refraction. 
By employing the particle sizing instrument described above and adding to it an optical system which collects the light scattered by each particle over the angular region of $40^{\circ}$ to $70^{\circ}$ in the plane of polarization it should be possible to classify those particles which are larger than $0.3 \mu \mathrm{m}$ as to whether they are nonabsorbing, carbon-1ike, or metallic. In somes cases, it also appears that some discrimination as to the type of metal might be possible.

In order to test the ability to distinguish between absorbing and nonabsorbing particles, a smoke detector based on a measurement of the intensity in the polarization plane from $40^{\circ}$ to $70^{\circ}$ was constructed. The primary method now employed for the detection of fire-produced aerosols involves monitoring a signal related to the total particle density. This technique has a disadvantage in that certain nonfire produced aerosols may have total particle densities high enough to cause confusion. These aerosols might be cigarette smoke, industrial dusts or fumes, or "hair spray" aerosols.

The smoke detector instrument contained a fairly straightforward optical system. Light from an incandescent source was polarized, collimated and impinged on an aerosol. Light scattered by the aerosol was monitored over two regions. One of these, $I_{11}$, was over the angular range from $40^{\circ}$ to $70^{\circ}$ in the plane of polarization. This quantity should be relatively large for nonabsorbing aerosols and should be less for aerosols containing absorbing materials. The second region was perpendicular to the polarization plane and over the angular range $80^{\circ}$ to $100^{\circ}$. This intensity, $I_{\perp}$, can be shown to be approximately proportional to the total number of particles in the beam regardless of whether they are absorbing or not. For display purposes, these two outputs have been applied to the vertical and horizontal axes of an oscilloscope, and the traces for a series of materials shown in figure 5. The length of the line is arbitrary and is related to how many particles are in the beam at any one time. As can be seen, $I_{11}$ is large relative to $I_{\perp}$ for nonabsorbing materials such as steam, cigarette smoke, and freon containing aerosols. However, for metal dusts and carbon containing materials $I_{\| 1}$ is small relative to $I_{\perp}$. Thus, a smoke detector which went into alarm on the slope of the $I_{11} / I_{\perp}$ curve rather than the total particle density could distinguish between fire-produced and nonfire-produced aerosols.

\section{REFERENCES}

[1] Gravatt, C. C., "Real time measurement of the size distribution of particulate matter in air by a light scattering method," J. Air Pollution Control Association, 23:12, 1035-1038 (Dec. 1973).

[2] Hodkinson, J. R., "Particle sizing by means of the forward scattering lobe," Appl. Opt.5:5, 839-844 (May 1966). 


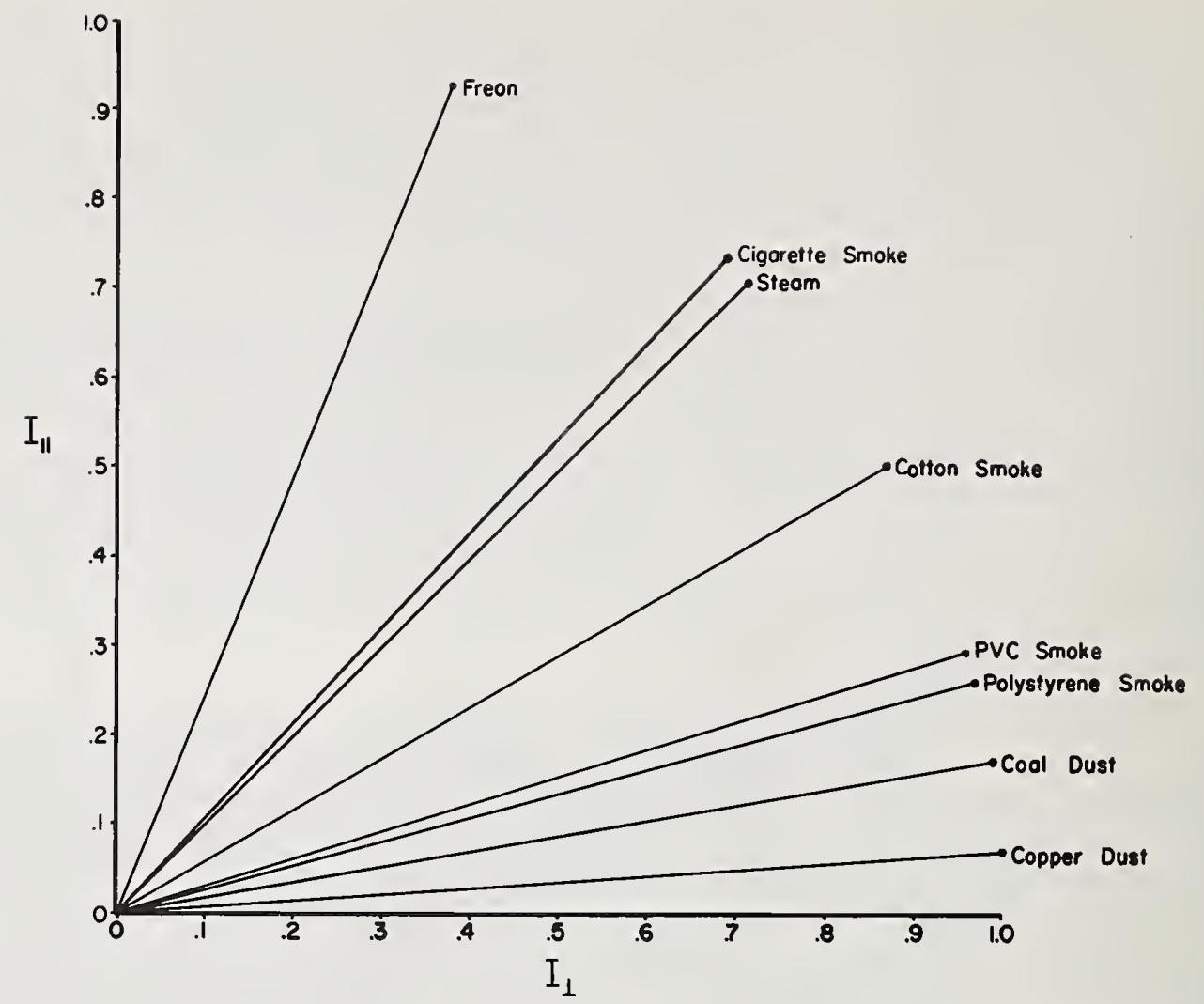

Figure 5. Smoke detector response. $I_{\| 1}$ and $I_{\perp}$ are defined in text. Length of line is arbitrary.

[3] Gravatt, C. C., and Allegrini, Ivo, A new Light Scattering Method for the Determination of the Size Distribution of Particulate Matter in Air, Proceedings of the 3rd International Clean Air Congress, p. C3 (1973).

[4] Greenough, M. L., and Gravatt, C. C., EAP Final Report on Particle Sizing Instrument (in preparation).

[5] Gravatt, C. C., and Allegrini, Ivo, Particle Sizing by Forward Lobe Techniques (in preparation).

[6] Gravatt, C. C., and Allegrini, Ivo, The Characterization of Particulates as to Their Optical Absorbing Properties (in preparation). 


\section{DISCUSSION}

GEORGE SINNOTT: In using two wavelengths to decrease the size do you mean that one would take the two wavelength scatterings separately?

GRAVATT: No. The laser is in the all-line mode so there will be some eight lines emitted by the laser--the three prominent ones are the $448.0,514.5$ and $640.0 \mathrm{~nm}$. These calculations are done weighing these intensities appropriately. It is assumed that all the light is collected and it results in a smcothing of the ripples of the response curve shown for a single wavelength.

RONALD NELSON: Going back to your calculation of the ratio as a function of the particle size in the envelope, my question is, when it gets down to the practical business of calibrating, how does one choose the center of that envelope for the real particle calibration?

GRAVATT: Let's go back to figure 3 and it will probably be easier to see what is involved. For instance, we have calculated a large number of these curves to make up the envelope. There is one for polystyrene latex, particles the index of those is 1.59. Using that curve and the instrument output for a number of polystyrene latex samples it is possible to transform the data from a relative scale to an absolute size scale. It is a problem to generate monodisperse aerosols, however. There is not a large number of well standardized or well calibrated latex or aerosol standards. So calibrating any particle sizing instrument is going to be somewhat difficult.

NELSON: I think that this ought to be considered as to how does one define this. I think that maybe we ought to advocate using the full width of this envelope as opposed to maybe half-width because if you are going to pick half it implies you know where the middle of it is and that is not necessarily the case.

GRAVATT: Well, as far as stating errors is concerned, one can report the full width or the half width. I do not feel that it is difficult to define where the middle of the curve is. It does not seem to me to be a problem that the particular calibration samples employed do not lie on the center line. However, since neither NBS or EPA have done an extensive calibration of the instrument I will have to withhold final comment until we get some experience and data. I personally feel that the most important problem in calibration is that of generating monodisperse aerosols.

ROBERT KNOLLENBERG: Did you say the mean particular size is 0.3 micrometers?

GRAVATT: The minimum resolvable particle size for a ratio if $10^{\circ}$ to $5^{\circ}$ is $.3 \mu \mathrm{m}$. You can detect particles down to about $0.1 \mu \mathrm{m}$. With 3 $1 / 3$ orders of magnitude gain in the electronics, if the large particle cutoff is set at approximately $3 \mu \mathrm{m}$. 
KNOLLENBERG: Do you know roughly what percentage of the total light scattering you collect?

GRAVATT: No, I don't know it exactly although I can calculate it. I would estimate that we collect several percent.

KNOLLENBERG: You are collecting a large percentage then.

GRAVATT: The EPA instrument has $1^{\circ}$ aperatures and this probably collects a few percent of the total scattered intensity.

NELSON: I guess it is about 1 percent.

GRAVATT: It may be 1 percent.

WILLIAM DORKO: Is this calculation dependent upon the structure of the particle such as spherical?

GRAVATT: All calculations are for spherical particles. It is stated that the forward lobe is least sensitive to particle shape. Dr. Kerker could probably make a better comment to that than I can. Hodgkinson also felt that way, but we have not done any calculations. We've tested nonspherical particles in the instrument, and they fit within the calibration curve. That's not really proof, it's only a suggestion. This is certainly an area where much more work is needed. 


\title{
FLOW APPARATUS FOR THE CHARACTERIZATION OF AEROSOLS
}

\author{
Madhav B. Ranade \\ Fine Particles Research \\ IIT Research Institute \\ 10 West 35 th Street \\ Chicago, Illinois 60616
}

\section{ABSTRACT}

A flow apparatus has been developed to enable the size measurement of liquid aerosols to be measured under defined conditions with the help of a light scattering technique. This apparatus was used to study the evaporative behavior of submicron aqueous aerosols during transportation in air. The apparatus is currently being used to characterize large ( $50 \mu \mathrm{m})$ aerosol particles under sedimentation. Portable versions of the flow apparatus are presently under development to characterize acid mist droplets, and to investigate the characteristics of therapeutic aerosols.

Key words: aerosol size measurements; aerosol spectrometer; aerosol sprays; condensation on aerosol droplets; evaporation of aerosol droplets; laser light scattering by aerosols; therapeutic aerosols.

\section{INTRODUCTION}

An aerosol system is characterized by the size distribution of the particles, concentration of the particles, and the physical nature of the particles. Physical properties of aerosols are strongly dependent on the particle size characteristics. Sampling and measurement techniques for liquid aerosol systems must be chosen with a regard to physical conditions. In order to obtain a representative sample from an aerosol system, changes in the temperature, pressure, and flow field during sampling must be minimized.

Volatile liquid aerosols pose a special problem, as the particle size may change significantly during sampling. Examples of such aerosols are the therapeutic aerosols and aerosol can sprays. In the therapeutic applications, the drug is dispersed in the form of aqueous solution. In the aerosol cans, the product is dispersed by spraying with a volatile solvent in the form of droplets up to $100 \mu \mathrm{m}$ in diameter. The particle size of the final residue may vary from submicronic to a few micrometers. 
The processes of evaporation and condensation are fast. As an example, the time for complete evaporation of a $1 \mu \mathrm{m}$ water droplet is less than one second.

Due to these unstable characteristics of volatile droplets, techniques for in situ monitoring of the aerosols are needed. These techniques must not disturb the aerosol. The choice of techniques to meet these requirements is limited. Optical visualization techniques such as in situ microscopy, photography, and holography are possible only for aerosols with particle size above 5 to $10 \mu \mathrm{m}$. Techniques based on angular light scattering and light extinction are useful for the submicron aerosols. The light extinction technique requires long path lengths which are not practical in the laboratory. Thus, techniques based on angular light scattering offer the only choice available for monitoring volatile liquid aerosols ranging from submicron to $50 \mu \mathrm{m}$ in particle size.

In the flow apparatus described here, the aerosol is monitored by light scattering in a controlled environment and can be used to study changing size characteristics of the aerosol.

\section{FLOW APPARATUS}

The volatile aerosol is injected isokinetically in a moving laminar uniform velocity air stream (fig. 1). The axial distance from the aerosol injection point is directly related to the age of the aerosol. If

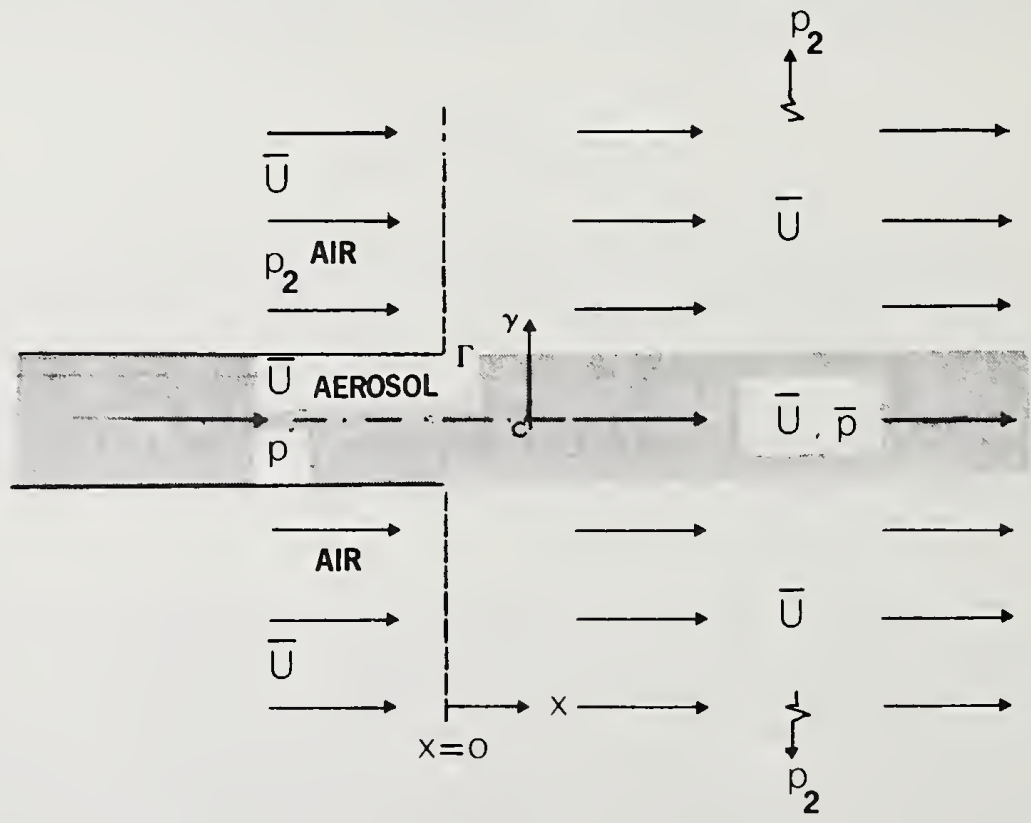

Figure 1. Aerosol jet injected isokinetically in a uniform laminar air stream. 
the vapor concentration of the volatile component is higher in the air stream than in the aerosol stream, the vapor will diffuse to the aerosol stream and the aerosol particles will grow by condensation. When the vapor concentration gradients are reversed, the aerosol particles will evaporate; and if the vapor concentrations are the same, the particles will be stable.

The flowing aerosol can be conveniently monitored by a light scattering technique at various axial positions, and since the axial position is directly related to the age of the aerosol, information about the rate of evaporation and condensation can be obtained.

An apparatus based on this principal was developed for studying the evaporation of $1 \mu \mathrm{m}$ sodium chloride solution aerosols under various humidities.* A three-inch diameter wind tunnel was constructed for this study (fig. 2). A specially designed entrance section was used to obtain a uniform laminar air flow in a three-inch diameter glass test

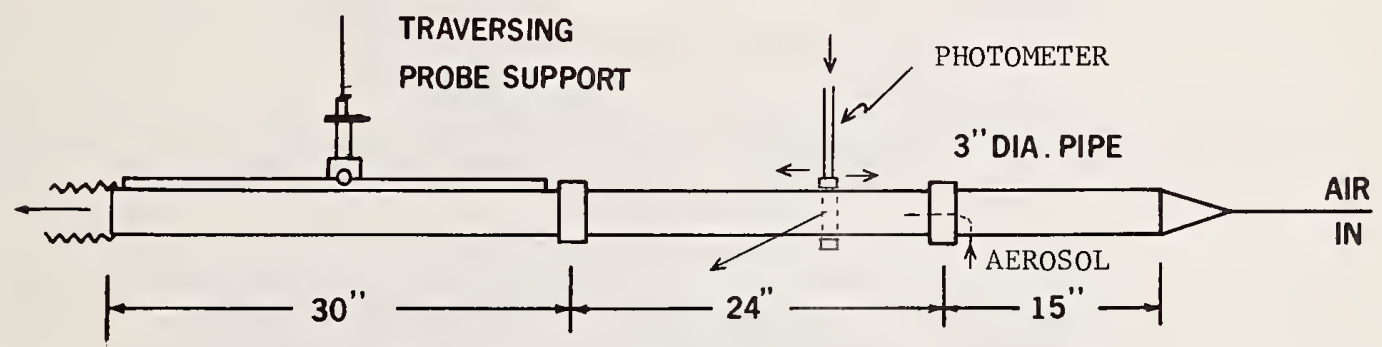

EXIT SECTION TEST SECTION ENTRANCE

SECTION

Figure 2. Wind tunnel for evaporation experiments.

section. The humidity of the air stream could be controlled by the mixing of a dry and a saturated air stream. The aerosol was generated from an aqueous sodium chloride solution ( $1 \% \mathrm{NaCl})$. The geometric number mean diameter of the aerosol particles was $1 \mu \mathrm{m}$ and a $\sigma_{\mathrm{g}}$ of 1.8 . A traversing $90^{\circ}$ light scattering photometer (fig. 3) was used to monitor the aerosol at various positions along the axis of the test section.

The aerosol stream at the injection point is saturated with water vapor. When the outer air stream was also saturated, no change in photometer response was observed. When the humidity in the outer stream was less than 100 percent, evaporation of particles was observed. The diffusion of the water vapor from the aerosol stream to the outer air stream was shown to control the size change of the aerosol. Using the

*Dynamic Properties of Submicron Aerosols, M. B. Ranade, Ph. D. Thesis, Illinois Institute of Technology, Chicago, 1974. 


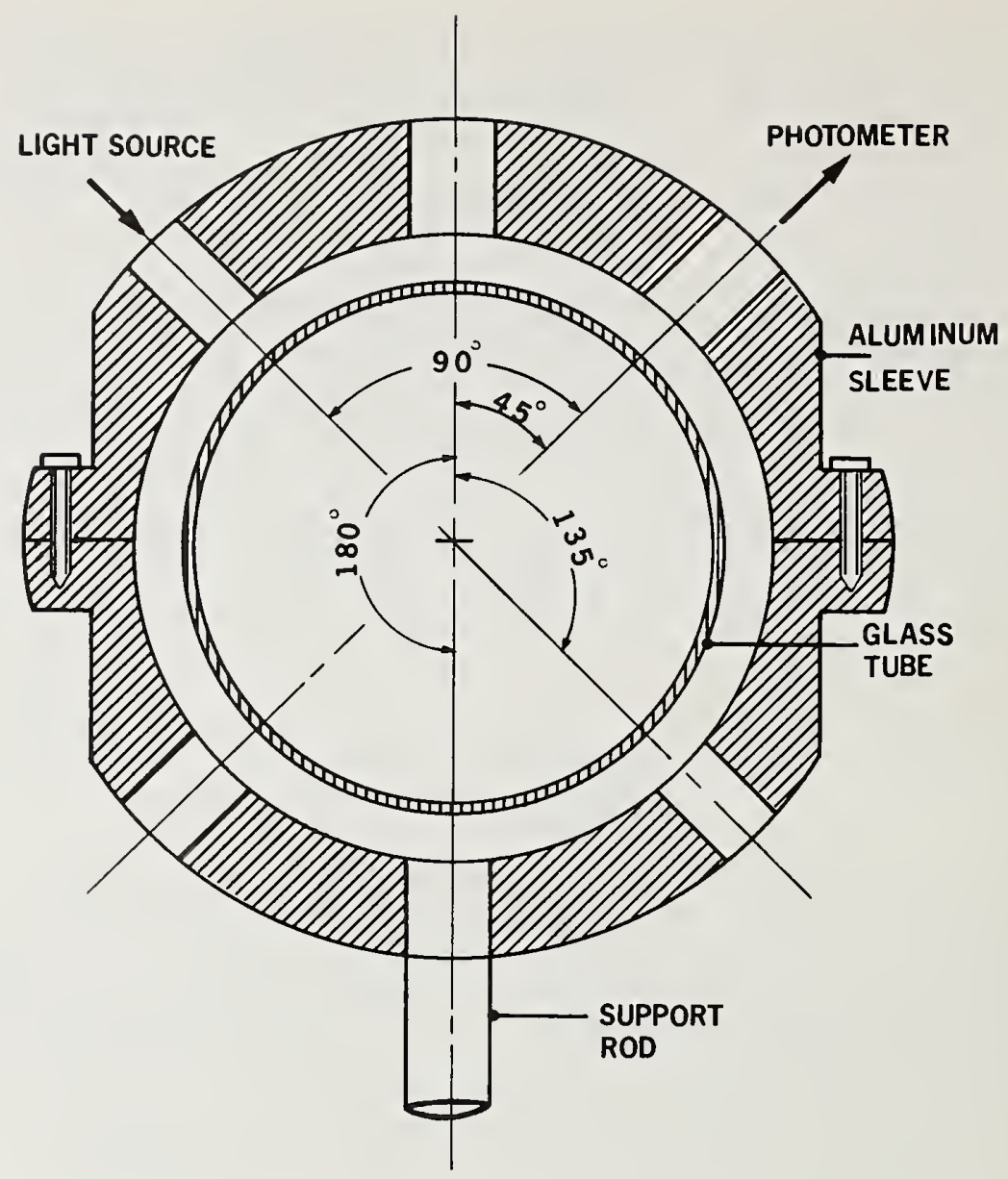

Figure 3. Traversing $90^{\circ}$ light scattering photometer.

diffusion theory and the Maxwell evaporation law, the size of the particles at various axial positions was predicted by computer calculations. The light scattering response at these axial positions was compared with the values calculated from the Mie Theory. As seen in figure 4, good agreement between the theory and the data was observed.

This apparatus has been modified for studying the evaporation of larger particles (up to $50 \mu \mathrm{m}$ ). The aerosol flows vertically downward and sedimentation of large particles out of the aerosol stream is avoided.

A portable version of the apparatus was constructed with a one-inch diameter glass tube (fig. 5). In this system, the aerosol can be pulled through the apparatus. If the air in the sample is used for sheath (outer air stream), the aerosol will be stable during observation. other light scattering geometries and techniques may be used for best sensitivity in various size ranges. Auxiliary apparatus for humidification and flow control are mounted on a trolley. 


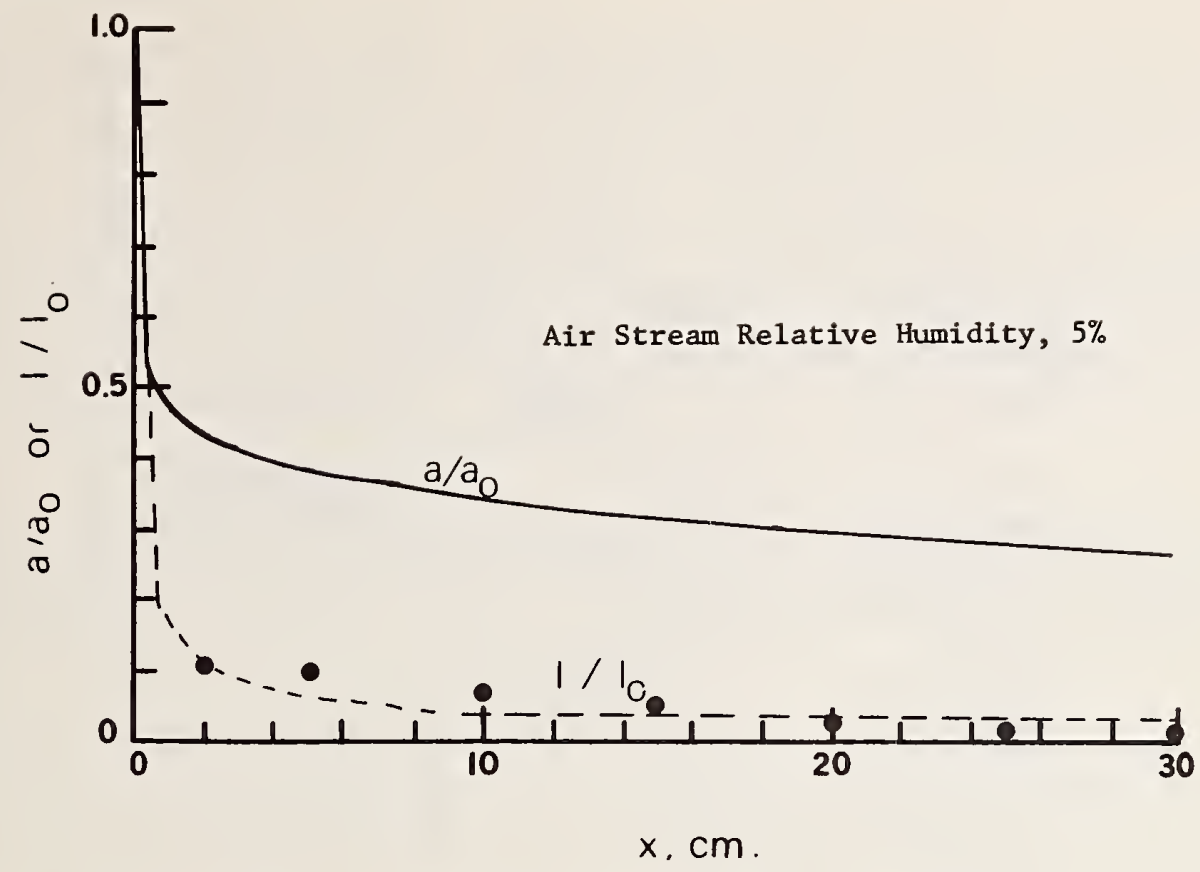

Figure 4. Evaporation of aqueous sodium chloride solution aerosol.

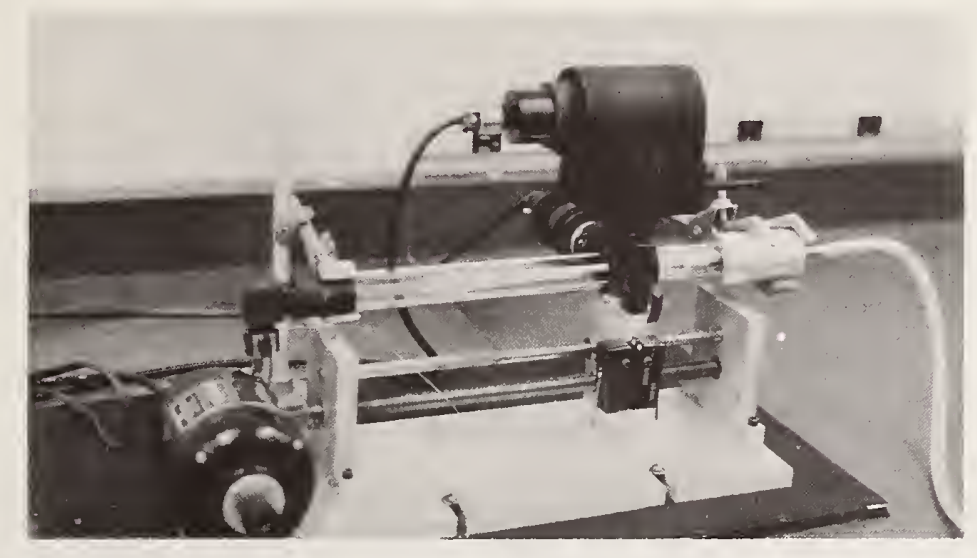

Figure 5. Portable flow apparatus.

At IITRI, we have set up a system for the monitoring of the liquid aerosols (fig. 6). The Cascade impactor is used with nonvolatile or volatile aerosols containing a solute. A differential mobility analyzer, developed by Dr. Earl Knutson, is used to obtain the size distribution of submicron residual aerosols too small for the impactor. A condensation nuclei counter is used to monitor the number concentration of the residual aerosol. The aerosol sample is pulled by a vacuum pump. A part of the sample air is filtered and used as a sheath air. The photometer is mounted on a motorized track utilizing a helical screw for traversing along the glass tube. 


\section{Liquid Aerosols}

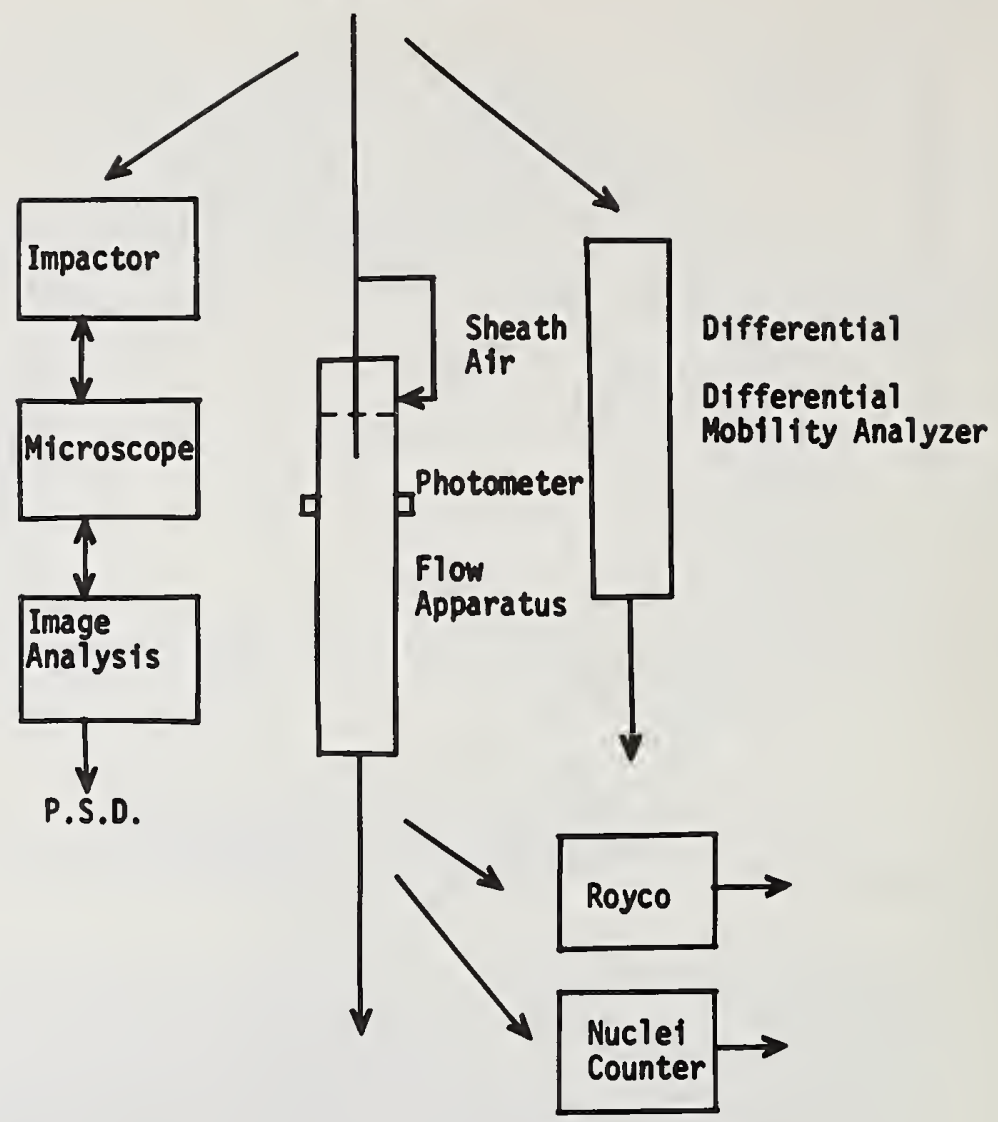

Figure 6. IITRI facility for characterization of volatile aerosols.

\section{SUMMARY}

A facility for the characterization of liquid volatile aerosols in the size range of 0.5 to $50 \mu \mathrm{m}$ has been established at IITRI. The heart of the facility is a flow apparatus. This apparatus was originally developed to monitor the evaporation of micron size aqueous aerosols.

We feel that the facility will be a convenient means for the characterization of volatile liquid aerosols such as the therapeutic aerosols and the aerosols from spray cans.

\section{SUMMARY OF THE MEASUREMENT TECHNIQUE}

The flow apparatus was originally constructed as a research apparatus to study the evaporation of micron size aqueous aerosols. The aerosol was injected isokinetically in the middle of a laminar uniform velocity air stream. The aerosol was monitored by traversing $90^{\circ}$ light scattering photometer at various axial positions, which were related to 
the age of the aerosol. The photometric data was reduced by the Mie scattering theory on a computer and showed good agreement with the theory for the evaporation of aerosols developed for this study.

The apparatus and a portable version of the same are being adapted to characterize volatile aerosols. The particle size measurement range is 0.5 to $50 \mu \mathrm{m}$, and the concentration range is 102 to 106 particles/ $\mathrm{cm}^{3}$. The aerosol is monitored in a controlled atmosphere without disturbance. The aerosol sampling rate is of the order of 1 to 5 liters/ minute.

The apparatus is currently under development and final specifications are yet unavailable. However, the cost of the apparatus is expected to be quite low, as most of the parts are simple and easily available.

\section{DISCUSSION}

ILAN CHABAY: Was most of the work you did with monodisperse systems?

RANADE: We worked with polydispersed systems which had a standard deviation of about 1.6 .

CHABAY: What sort of size range do you use?

RANADE: Essentially one micrometer.

GEORGE SINNOTT: Did you build your own mobility analyzer?

RANADE: Yes, Dr. Earl Knutsen built it. He has a differential mobility analyzer with a current measuring system.

MILTON KERKER: I have two questions. What is the holdup time and what is the concentration? And the second question, how do you handle the evaporation? Do you have to worry about the Kelvin effect and also I assume you consider the evaporation from a single particle. How do you handle a situation where you have a collection of particles?

RANADE: In response to the first question, the holdup time is the order of one to two seconds. That is a rather short time for coagulation to take place. The concentration is the order of 105 to $10^{6}$ per cubic centimeter which is not very high for micrometer size particles. Now for the second question, the Kelvin effect has been taken into account in the theoretical equation. One is essentially concerned with the vapor pressure of the water in equilibrium with the droplets. This gives rise to a solution effect and a Kelvin effect and both have been taken into account in solving the equations for the particle size. 
KERKER: There was an Aerosol Conference in Swan Sea last year, and the question of evaporation figured very centrally. As I recollect some people found that evaporation rates were much lower than they would have calculated from a simple hydrodynamic treatment of a single particle. Some people attributed this to impurities on the surface, but the thing that impressed me more than this was the fact that nobody seemed to come up with even a rough idea as to how the evaporation rate for a single particle would compare to that for a particle in an aerosol mixture. wonder if you have any thoughts on that.

RANADE: Yes, in an aqueous aerosol, evaporation is contributing water vapor to the system so the humidity is changing. For my aerosol at one micrometer size and at a concentration of $10^{6}$ the contribution to the humidity wasn't very large. I found that it could be treated the same as single particles. Now if you had a denser system, one would have to build something like a cell model. I don't know whether that is justified or not, but a cell model means that instead of assuming that the particle is surrounded by an infinite media, we consider an average interparticle distance and solve the equations accordingly. You can show for this concentration the correction for evaporation will be very low.

KERKER: There is one thing that always bothers me with water aerosols. You say the evaporation doesn't contribute very much to the humidity, conversely you need fantastic humidity control in order to define just what is happening with respect to evaporation.

RANADE: Yes, depending on which end of the scale you are working. For example, my calculations were based on $10^{6}$ particles per cc. Assuming complete evaporation of one micrometer particles, the contribution to the relative humidity was one percent. On the other hand when the relative humidity exceeds 85 percent the growth curve is very steep, and a small change can be quite important.

REG DAVIES: Dr. Morton Corn at Pittsburgh has been doing some work with sulphuric acid aerosols in the range of 95 percent relative humidity. Here he observes an enormous change in growth corresponding to a change of $1 / 2$ percent relative humidity, so this control of humidity is an important factor.

WENDELL ANDERSON: I think the same phenomenon was reported 20 years ago by LaMer.

DAVIES: We've done a literature search on this and there have been quite a number of reports about size growth versus humidity, especially for things like sodium chloride aerosols in lungs which have been used to investigate lung humidity.

CHABAY: We did some work on cloud droplet growth and we saw some rather strange results at very low levels of supersaturation. The exact values of supersaturation were extremely critical in determining growth rate of the droplets falling through a temperature gradient. 
Thomas R. Marsha11, Charles S. Parmenter, and Mark Seaver

Department of Chemistry

Indiana University

Bloomington, Indiana 47401

\section{ABSTRACT}

Nearly complete $360^{\circ}$ scattering diagrams have been obtained from each of a large number of individual aerosols which stream in an air sheath through a laser beam. A computer analys is of diagrams from a "monodisperse" aerosol (polystyrene spheres with mean diameter near 1.2 $\mu \mathrm{m})$ shows that both diameter and refractive index can be determined from each scattering diagram to compile a statistical characterization of the aerosol. Diameter precision is about 0.4 percent and the refractive index can be determined to within 0.7 percent.

The method is clearly a practical approach to routine characterization of aerosol sprays containing spherical particles whose diameters range from about $0.3 \mu \mathrm{m}$ to at least $20 \mu \mathrm{m}$. The refractive index determination will reveal differences between particle composition and bulk composition of the parent material as well as composition changes during aging of aerosol sprays.

Key words: aerosol light scattering; aerosol size measurements; aerosol spectrometer; aerosol sprays; laser light scattering by aerosols; refractive index; scattering diagrams; $360^{\circ}$ scattering by particles.

\section{INTRODUCTION}

Resolved $360^{\circ}$ light scattering diagrams from individual particles contain sufficient information to determine the size, the index of refraction, and, in principle, the shape of regular aerosol particulates. Such data offers an attractive route for characterization of aerosols, but unfortunately an experimental barrier exists. Most instruments capable of these measurements require levitation of a particle for periods of at least minutes while $360^{\circ}$ scans are made [1-5], so that

*Contribution No. 2389 from the Chemistry Department, Indiana University. This work has been supported by NSF Grant GP 38275 . 
acquisition of data on statistical numbers of particles in an aerosol is not routinely practical.

Professor Frank Gucker and his colleagues at Indiana University have developed an instrument that obviates this problem $[6,7]$. They have reduced data collection times by four orders of magnitude so that $360^{\circ}$ diagrams can be made while particles stream through a laser scattering beam.

In this report we describe the initial calibrations and trials of this instrument using spherical particles of about one $\mu \mathrm{m}$ diameter. Our first results show clearly that the instrument represents a practical approach for statistical characterization of an aerosol comprised of spherical particles of unknown diameter $(0.5$ to $20 \mu \mathrm{m})$ and unknown refractive index.

\section{The Instrument}

Details of the instrument's optical design and mechanical construction are given elsewhere [7]. A schematic of $i$ ts operating principle is given in figure 1. In short, an air stream containing aerosol particles intercepts a He-Ne laser beam at one of the focii of an elliptical mirror which directs a $360^{\circ}$ slice of scattered light to a photomultiplier at the other focus. Angular discrimination is introduced by a $5^{\circ}$ rotating aperture between the scattering plane and the photomultiplier. A nearly complete $360^{\circ}$ scattering diagram is obtained in about $20 \mathrm{msec}\left(7^{\circ}\right.$ are missing either side of $0^{\circ}$ and $\left.180^{\circ}\right)$. The particle is effective stationary in the laser beam during this time. Scattering diagrams are displayed on an oscilloscope and can also be stored on magnetic tape by an interface with an XDS Sigma II computer.

\section{EXPERIMENTAL SCATTERING DIAGRAMS}

Al1 of the data described in this report have been obtained from polystyrene spheres in Dow aerosol sample LS-1028-E. This is a "monodisperse" aerosol with mean particle size reported by the manufacturer to be $1.099 \pm .0059 \mu \mathrm{m}$ and with a refractive index of 1.59 .

Figure 2 shows an experimental scattering diagram obtained from a single particle as it streamed through the laser beam. The diagram is plotted from data in computer storage. The mirror symmetry of the scattering pattern is especially significant because it proves to be a sensitive indication of an accurate scattering diagram.

A test of the reproducibility of the data is offered in figure 3 where four scattering curves from the same particle are compared. These curves were obtained in successive scans as the particle traversed the $1.5 \mathrm{~mm}$ cross section of the laser beam. Such reproducibility is typical of the instrument. In a size analysis by computer (see below), these four curves were assigned to particles with diameters $1.180,1.180$, 


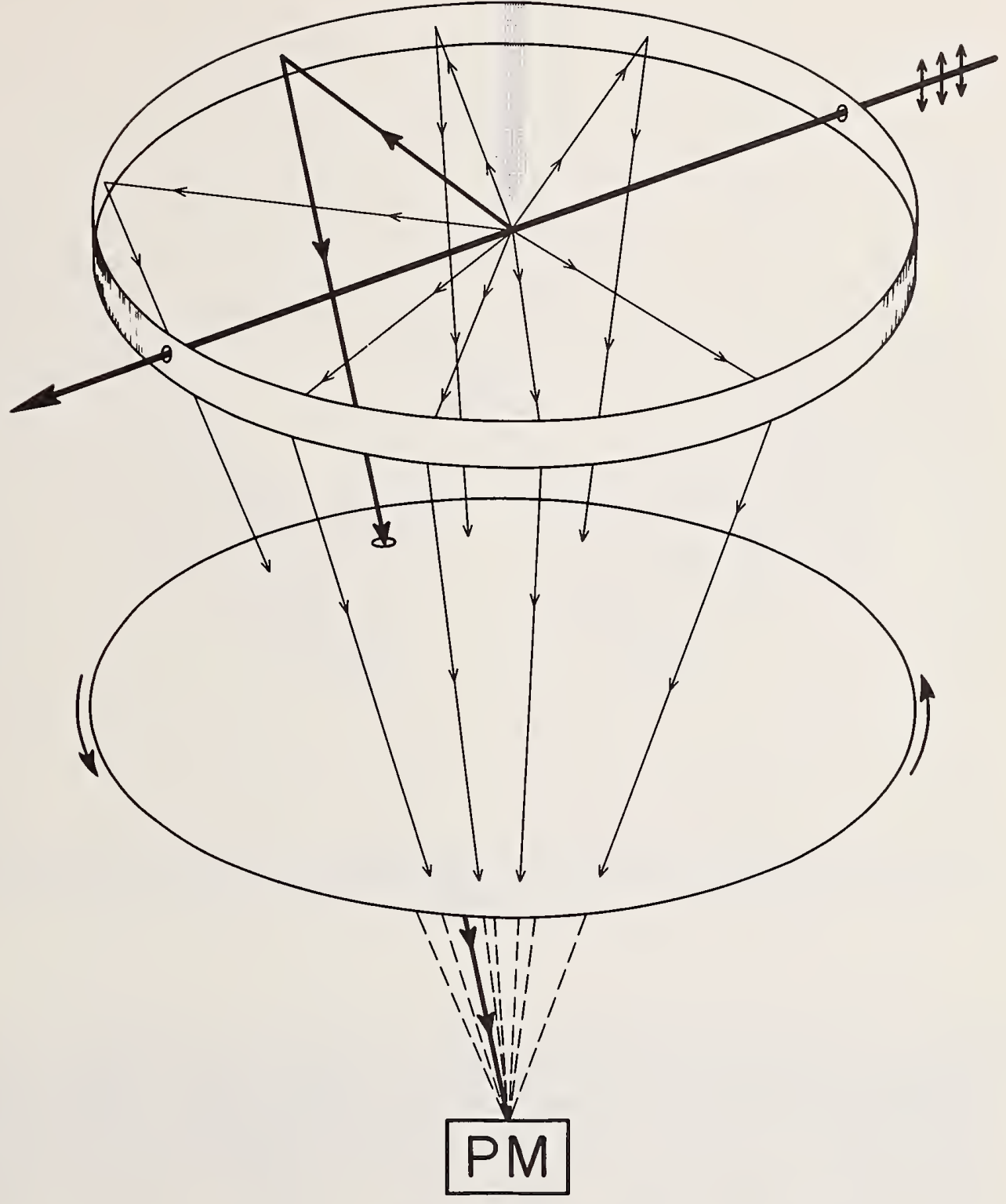

Figure 1. A schematic of the $360^{\circ}$ scattering instrument. An aerosol stream intercepts a He-Ne laser beam ("vertical" polarization) at one of two foci of an ellipsoidal mirror. Light scattered in the "horizontal" plane is intercepted by a segment of that mirror and directed to a photomultiplier at the second focus. A rotating aperature allows only a $5^{\prime \prime}$ segment of the scattered light to be detected at any given time by the photomultiplier. The aperature rotates at about $3000 \mathrm{rpm}$ so that a $360^{\circ}$ scan is completed in 20 msec. The photomultiplier signal comprising a $360^{\circ}$ scattering diagram if fed through a logarithmic amplifier to a computer for storage and processing. 


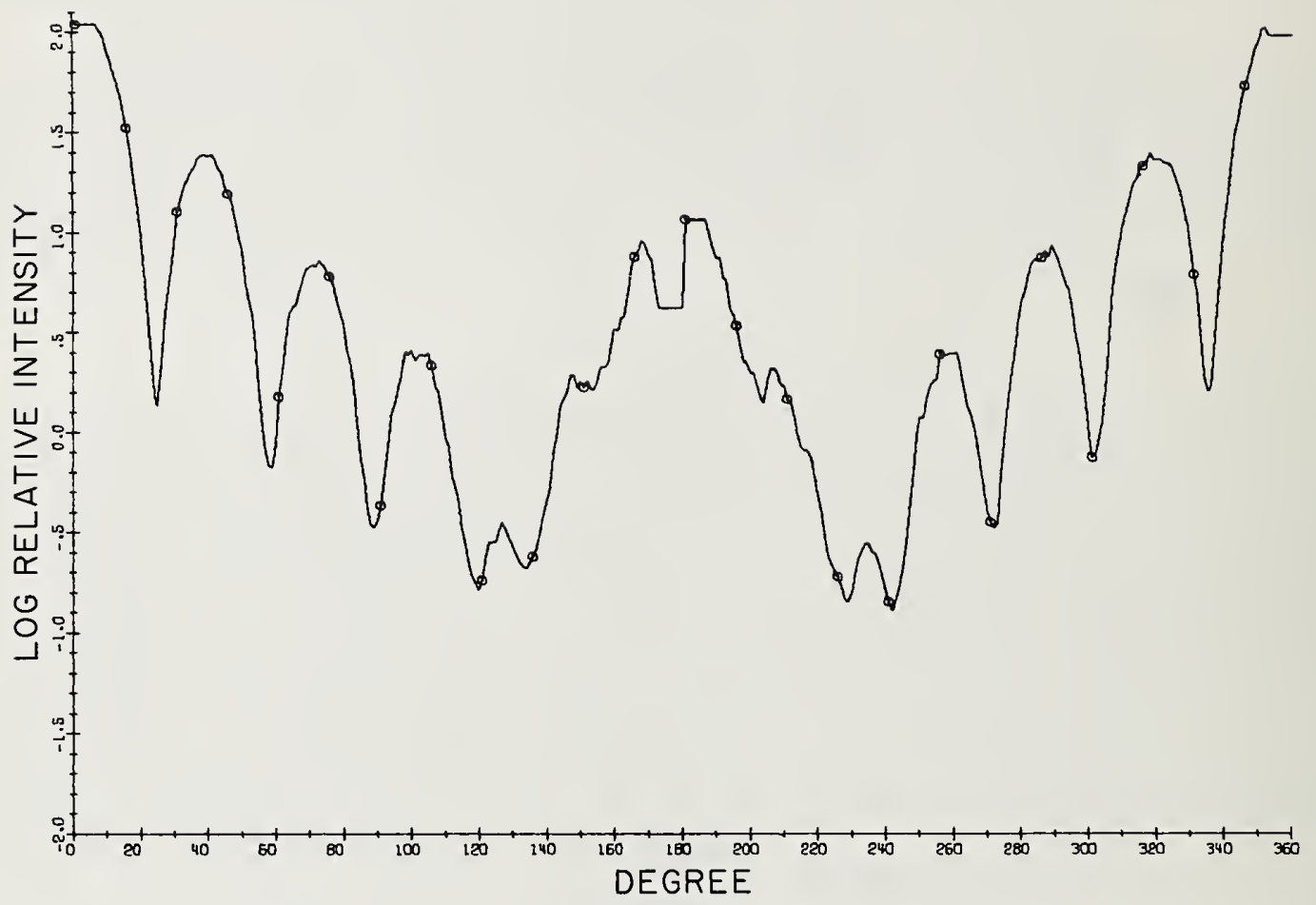

Figure 2. A scattering diagram obtained from the instrument described in figure 1 as plotted from computer storage. This diagram is from a polystyrene sphere about $1.190 \mu \mathrm{m}$ in diameter. The signal is blocked for $7^{\circ}$ either side of $0^{\circ}$ (forward) scattering and $7^{\circ}$ either side of $180^{\circ}$ scattering. The relative intensity scale is logarithmic and accurate at intensities $\simeq-0.2$. Below that value, limited bandwidth of the log amplifier has distorted the data. 


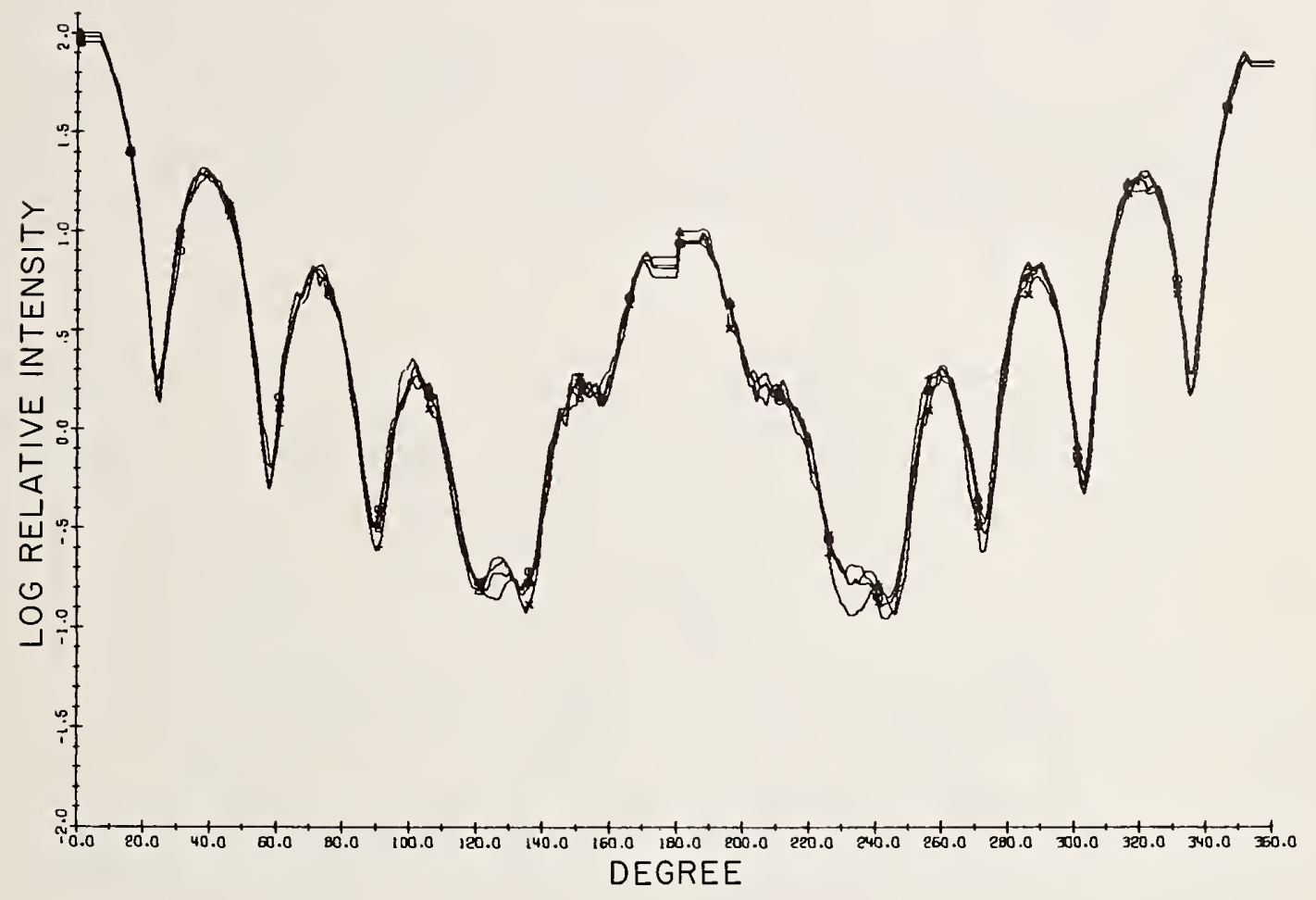

Figure 3. Four experimental scattering diagrams from the particle. These were obtained sequentially as the particle was in transit across the laser beam. 
1.180 and $1.175 \mu \mathrm{m}$. The noise in these diagrams is thus sufficiently low to allow size determinations to within about 0.5 percent.

\section{COMPARISON WITH THEORY}

A comparison of the experimental scattering diagram with a theoretical Mie scattering diagram is shown in figure 4 . The theoretical curve was computed for a particle of $1.190 \mu \mathrm{m}$ diameter using a program provided by Milton Kerker. The index of refraction was set at 1.59. The latter value is taken from the manufacturer's statement. The size was chosen by trial as the most satisfactory visual fit to the data. The mismatch between the curves at relative intensities below -0.2 is due to the restricted low-signal bandwidth of the log amplifiers servicing the photomultipler.

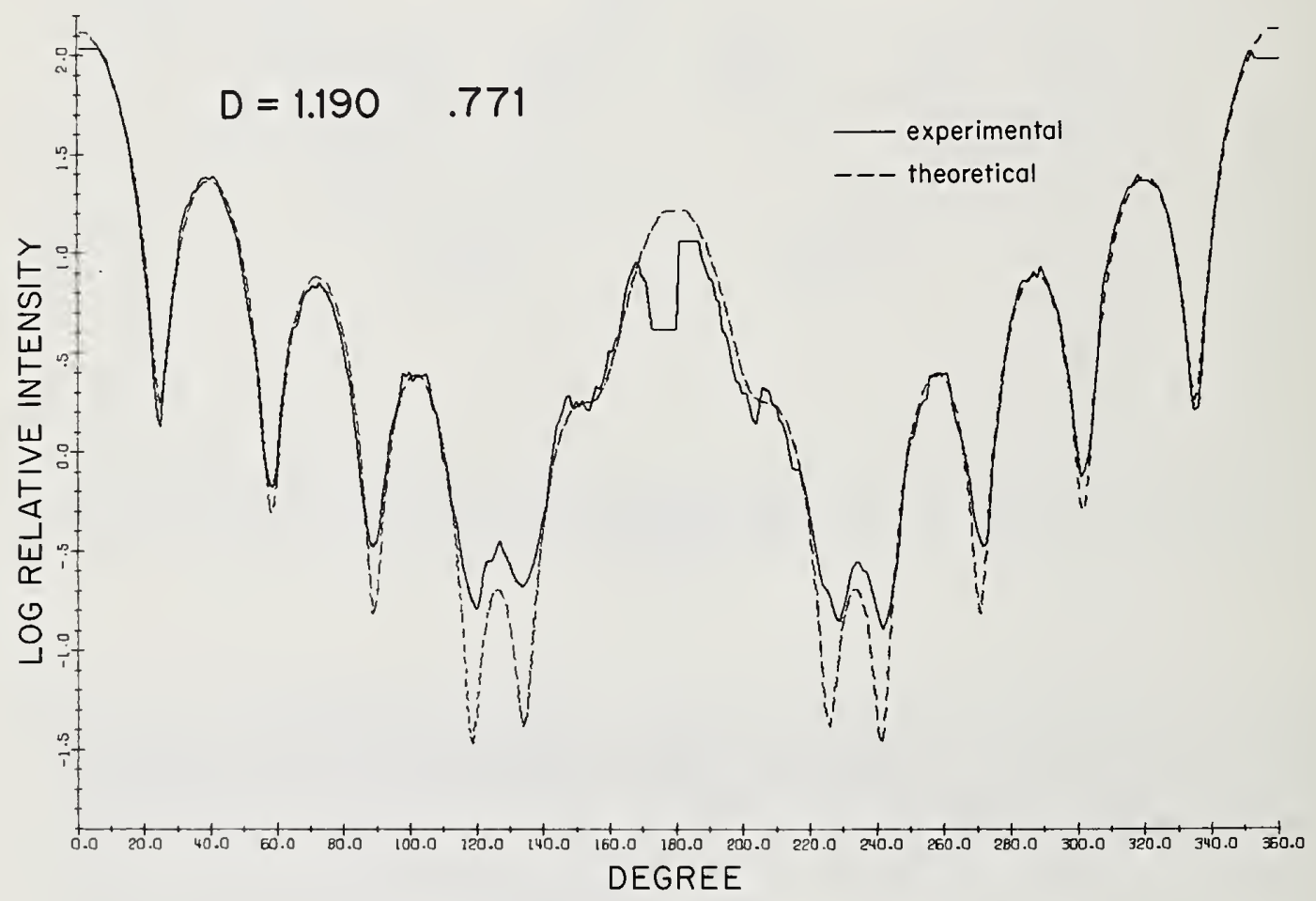

Figure 4. A comparison of theoretical and experimental scattering curves. The experimental curve is taken from figure 2 and the theoretical curve is computed for a particle with $1.190 \mathrm{~m}$ diameter and $m=1.59$. The theoretical curve is degraded to correspond to experimental data with the $5^{\circ}$ aperture and blanked-out at $0^{\circ} \pm 7^{\circ}$ and $180^{\circ} \pm 7^{\circ}$. Mismatch between the curves at relative intensities below -0.2 is due to distortion of the experimental curve during amplification of the photomultiplier signal. 
Such visual comparisons form one method of size determination. The fit in figure 4 is typical in quality to all comparisons we have made using particles from Dow aerosol LS-1028-E. Visually, one can sense distinct preferences for a "best" trial fit only when size increments used for the calculated curves become somewhat greater than $0.01 \mu \mathrm{m}$. Thus it is probably accurate to say that visual comparisons of theoretical with experimental diagrams in this size range can yield particle size to within about $0.015 \mu \mathrm{m}$ or 1.3 percent.

These points are illustrated in figure 5 where the experimental curve of figure 2 is compared with less optimal theoretical curves. By restricting attention to relative intensities greater than -0.2 (where the data are valid), one can see that in this size range the sensitivity to particle size appears predominantly in the $90^{\circ}$ sectors adjacent to backward scattering.
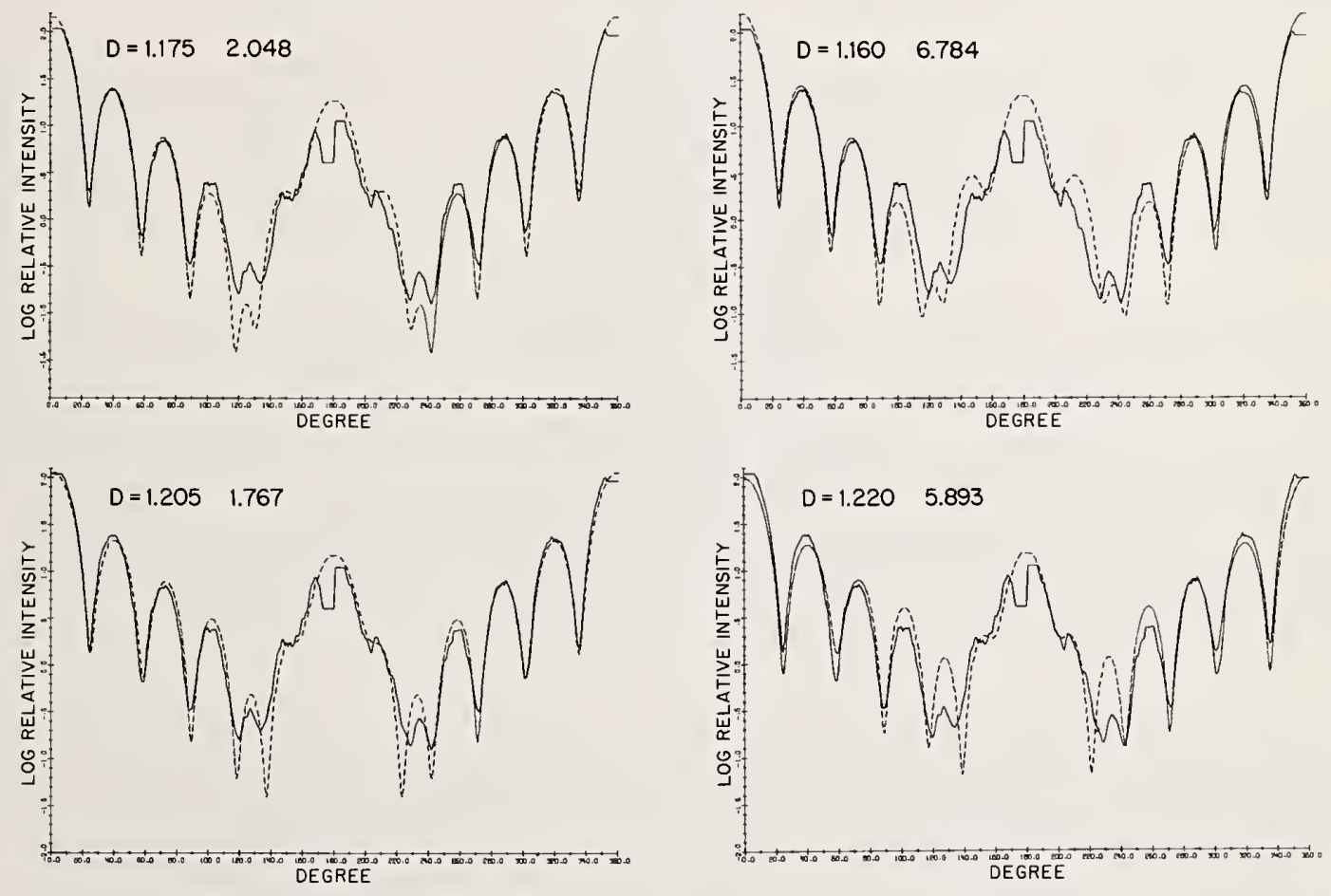

Figure 5. Comparisons of the experimental curve in figure 2 with theoretical curves calculated for particle sizes departing from the best fit $(D=1.190 \mu \mathrm{m})$ displayed in figure 4 . Theoretical curves are shown by dotted lines. The upper curves show decrements from optimal fit by analogous changes; $0.015 \mu \mathrm{m}$ (1eft) and $2 \times 0.015 \mu \mathrm{m}$. The assumed diameter $D$ is shown for each comparison, and the number to the right of the diameter describes the error of fit (least mean squares) for intensities $\geq-0.2$. A choice of particle size from the five comparisons in figures 4 and 5 is seen by this criterion as well as by visual fit to be $1.190 \mu \mathrm{m}$. 
Such comparisons as described above are impractical by dint of tedium for routine sizing of enough particles to yield a statistically significant analysis. A computer has been used to automate the analysis. The computer first checks the mirror symmetry of the experimental diagram. Nonspherical or improperly measured aerosols are revealed by asymmetric scattering. The symmetric diagrams are then compared to a library of theoretical curves representing various particle sizes. A least mean square analysis chooses the best fit and hence determines the particle size.

A check of the computer analysis has been made by comparison of visual and computer size determinations on a sample of 95 particles using $0.015 \mu \mathrm{m}$ size increments. The agreement was satisfactory.

Computer fitting also yields a more precise determination of size. Computer fitting can discriminate between alternate choices of theoretical curves differing by only $0.005 \mu \mathrm{m}$. This is about 0.4 percent of particle size and represents the practical resolution of our apparatus in this range.

A computer analysis of Dow aerosol sample LS-1028-E was made with data collected from 180 particles. Diagrams from 63 of these were computer rejected on the basis of asymmetric scattering. (The rejected data are diagrams of dust, multiple particles, excessive noise, particles exiting from the beam during recording of diagrams and so forth.) The remaining diagrams on 117 particles were explored by computer for best fit to theoretical diagrams with trial increments of $0.005 \mu \mathrm{m}$ and an index of refraction $m=1.59$. The subsequent listing of particle diameters is plotted in figure 6 .

This analysis yielded a mean diameter of $1.188 \pm 0.007 \mu \mathrm{m}$. The mean diameter is about ten percent larger than the value reported by the manufacturer, but it agrees well with the values reported in seven other explorations of this standard. The data are summarized in table 1.

As a side issue, we show in figure 7 a comparison of the experimental curve from a $1.190 \mu \mathrm{m}$ diameter particle (close to the mean diameter) with the theoretical scattering curve for the mean particle size given by the manufacturer $(1.099 \mu \mathrm{m})$. There is hardly a danger of confusing the issue with $360^{\circ}$ scattering curves.

\section{Particle Size and Index of Refraction}

The most intriguing promise of this method of aerosol characterization lies in its ultimate ability to define particle shape and index of refraction in addition to the size measurements described above. No steps have yet been taken to observe scattering from nonspherical parti- 


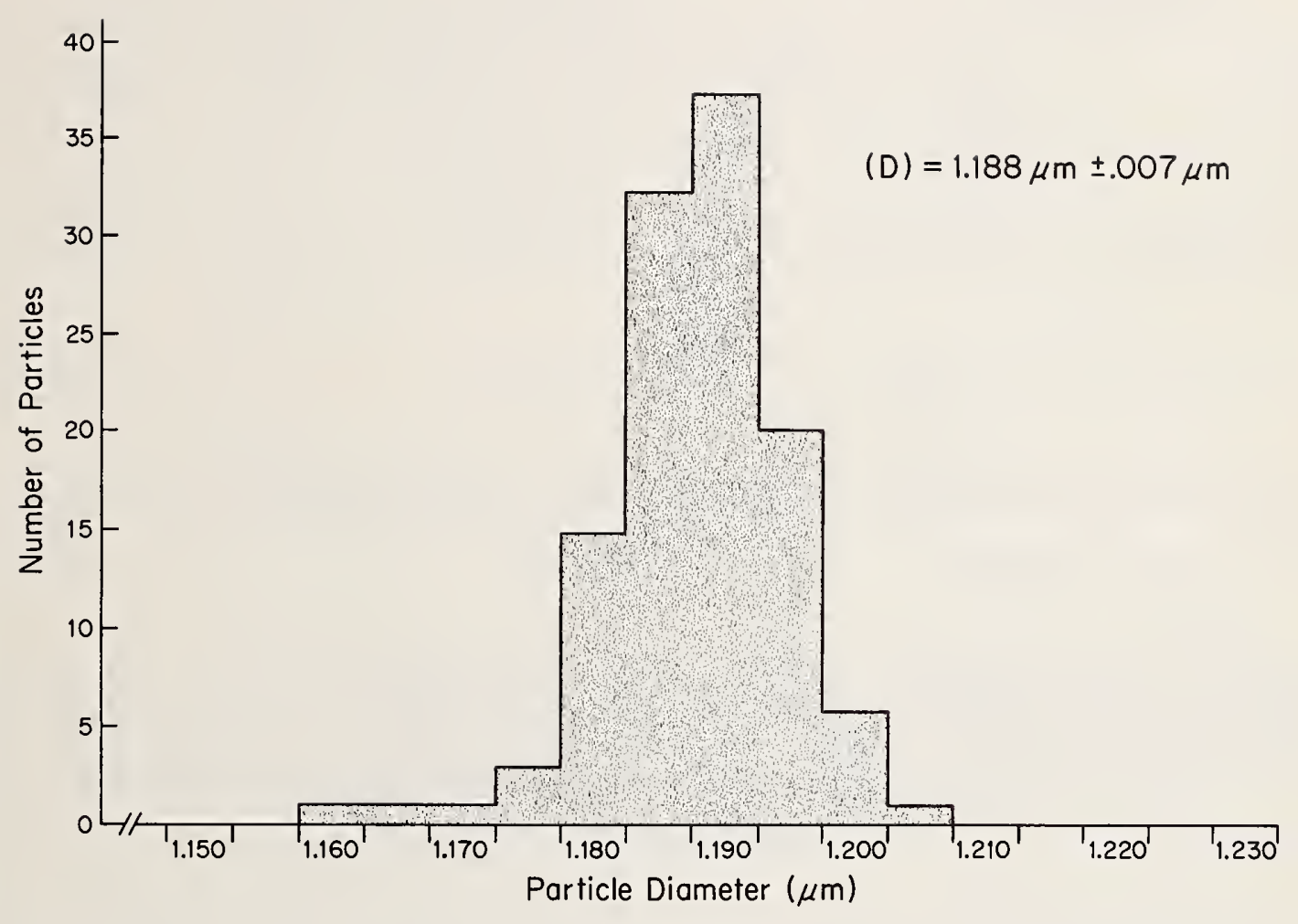

Figure 6. The number distribution of particles sizes in Dow aerosol sample LS-1028-E determined by computer comparison of experimental $360^{\circ}$ scattering diagrams with calculated Mie diagrams. The refractive index was assumed to be 1.59 and the assumed diameters for calculated curves were varied in $0.005 \mu \mathrm{m}$ increments. 
Table 1. Reported values of analysis of Dow aerosol standard sample LS-1028-E.

Reference

Method

m.d. \pm S.D. $(\mu \mathrm{m})$

Dow

Electron microscope

$1.099 \pm 0.0059$

Heard et at. $(1970)^{a}$

Electron microscope

$1.16 \pm 0.016$

Stober and Flachsbart

Electron microscope

$1.26 \pm 0.032$

$(1971)^{d}$

Portsendorfer and Heyder Electron microscope

$1.172 \pm 0.027$

$(1972)^{\mathrm{C}}$

Phillips et al. $(1970)^{b} \quad$ Scattering from single particle

$1.200 \pm 0.010$

Stober and Flachsbart

$(1971)^{d}$

Spiral centrifuge

$1.22 \pm 0.018$

Wims and Meyers (1972) $)^{e} \quad$ Scattering from solution

$1.20 \pm 0.012$

Gucker et a1. (1973) ${ }^{f}$

This work

Scattering from single particle $1.196 \pm 0.0065$

Scattering from single particle $1.188 \pm 0.007$

$a_{\text {M.J. Heard, A.C. Wells, and R.D. Wiffen, Atmospheric Environment }} 4$. 149 (1970).

D.T. Phillips, P.J. Wyatt, and R.M. Berkman, J. Colloid Interface Sci. 34, 159 (1970).

c J. Porstendorfer and J. Heyder, J. Aerosol Sci. 3, 141 (1972).

$d_{W}$. Stober and H. Flachsbart, J. Aerosol Sci. 2, 103 (1971).

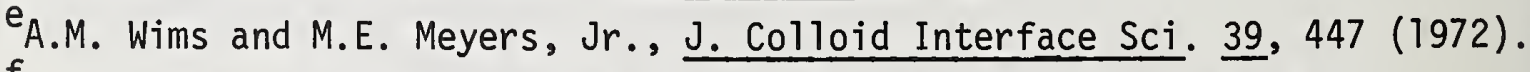

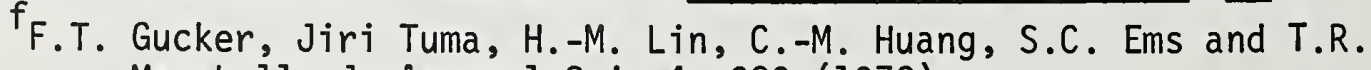
Marshal1, J.Aerosol Sci. 4, 389 (1972). 


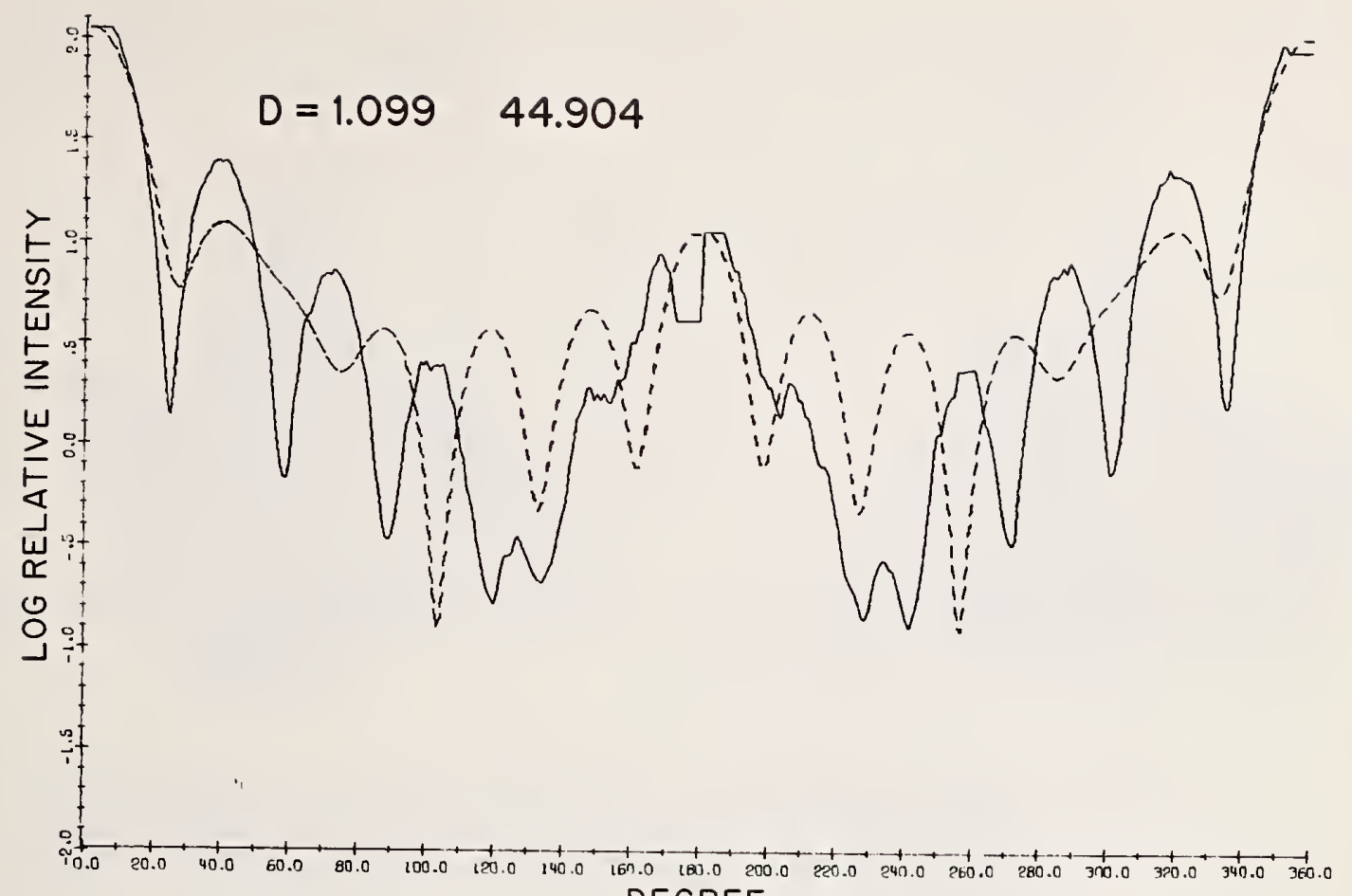

DEGREE

Figure 7. A comparison of the experimental scattering curve (solid line) for a $1.190 \mu \mathrm{m}$ particle (near the mean diameter of particles Dow aerosol LS-1028-E) with a curve calculated for diameter 1.099 $\mu \mathrm{m}$. The latter is the mean size reported by the manufacturer.

cles, but the initial explorations of simultaneous size and index of refraction determinations are at hand.

We have taken the experimental scattering diagrams of seven particles from Dow aerosol LS-1028-E and searched for the best computer fits to theoretical curves in which both $\mathrm{m}$ and $\mathrm{D}$ were varied parametrically. Diameters, $D$, were incremented by $0.005 \mu \mathrm{m}(0.4 \%)$ and the refractive index, $m$, was incremented by 0.01 (about $0.9 \%$ ).

Computer selections of $m$ and $D$ for these particles are summarized in table 2. The index of refraction was found to be constant for six of these particles and was chosen as an adjacent increment for the seventh. Thus the technique is successful in the recognition of an assembly of particles with uniform index of refraction but dispersion in size.

The fitting procedure is illustrated in table 3 which displays a matrix of least mean square deviations between theoretical trials and an experimental curve for particle No. 7 (which is also the curve in figure 2). Better fits are represented by smaller numbers. This is the only 
Table 2. Characterizations of particles in Dow aerosol LS-1028-E by computer fit of calculated Mie diagrams to $360^{\circ}$ experimental diagrams.

\begin{tabular}{|c|c|c|}
\hline Particle & Index of $_{m} \underset{m}{\text { refraction }}{ }^{\mathrm{a}}$ & Diameter $^{\mathrm{a}}$ \\
\hline $\begin{array}{l}1 \\
2 \\
3 \\
4 \\
5 \\
6 \\
7\end{array}$ & $\begin{array}{l}1.59 \\
1.59 \\
1.59 \\
1.59 \\
1.59 \\
1.59 \\
1.58\end{array}$ & $\begin{array}{l}1.190 \\
1.190 \\
1.190 \\
1.195 \\
1.195 \\
1.195 \\
1.195\end{array}$ \\
\hline
\end{tabular}

$a_{m}$ and $D$ were varied parametrically in trial Mie diagrams with increments of 0.01 and $0.005 \mu \mathrm{m}$ respectively.

Table 3. Least mean square deviations between the experimental scattering diagram of the particle in Figure 2 and calculated Mie diagrams for various values of $\mathrm{m}$ and $\mathrm{D}$. Best fit for this particle is seen to be $m=1.58$ and $D=1.95$ is in $\mu \mathrm{m}$.

\begin{tabular}{c|ccccccccc}
\hline m & 1.55 & 1.56 & 1.57 & 1.58 & 1.59 & 1.60 & 1.61 & 1.62 & 1.63 \\
\hline 1.170 & 17. & 13. & 8.3 & 5.1 & 3.1 & 2.3 & 2.4 & 3.0 & 4.0 \\
1.175 & 14. & 9.6 & 5.9 & 3.4 & 2.0 & 1.7 & 2.0 & 2.8 & 3.9 \\
1.180 & 11. & 7.0 & 4.0 & 2.2 & 1.3 & 1.3 & 1.8 & 2.7 & 4.0 \\
1.185 & 8.4 & 5.0 & 2.7 & 1.4 & .93 & 1.1 & 1.8 & 2.9 & 4.4 \\
1.190 & 6.3 & 3.5 & 1.8 & .93 & .77 & 1.2 & 2.1 & 3.4 & 5.2 \\
1.195 & 4.6 & 2.5 & 1.3 & .75 & .85 & 1.5 & 2.6 & 4.3 & 6.6 \\
1.200 & 3.5 & 1.9 & 1.02 & .81 & 1.2 & 2.1 & 3.6 & 5.7 & 8.7 \\
1.205 & 2.7 & 1.5 & 1.03 & 1.1 & 1.8 & 3.0 & 5.0 & 7.8 & 12. \\
1.210 & 2.2 & 1.4 & 1.3 & 1.7 & 2.7 & 4.5 & 7.1 & 11. & 16.
\end{tabular}

one of seven diagrams so far explored in which the refractive index was found to be other than 1.59. Even in this case, however, the choice between $m=1.58$ and 1.59 is ambiguous. Trial curves for each fit almost equally well. Deviations from the region of optimal $\mathrm{m}$ and $\mathrm{D}$ quickly become large, and our experience has been that a unique choice of $\mathrm{m}$ and $\mathrm{D}$ is generally derived.

Figure 8 shows the matrix of table 3 plotted as a contour map. 


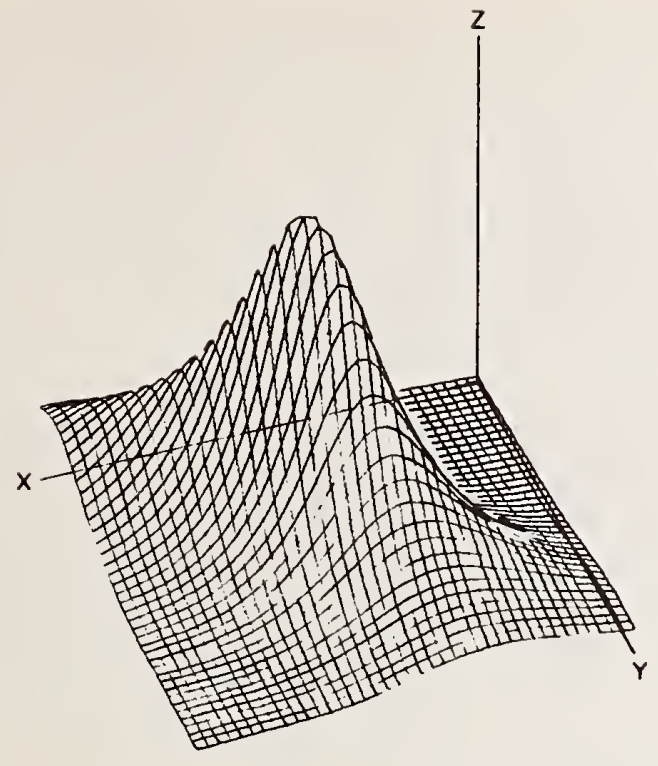

VIEWING ANGLE

THETA $=50.00$

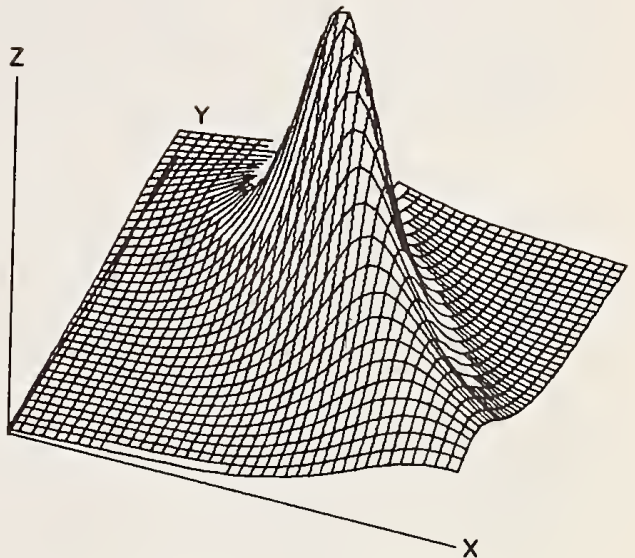

VIEWING ANGLE

THETA $=50.00$

$\mathrm{PHI}=290.00$

Figure 8. Three dimensional perspective views of the curve fits listed in table 3 . The curve deviations ( $Z$ coordinate) have been inverted so that the best fit appears as a contour peak. The coordinate origins are $x_{0}=1.140 \mu \mathrm{m}$ (diameter $D$ ) and $y=1.530$ (refractive index $\mathrm{m}$ ). The contour lines are incremented by $0.0025 \mu \mathrm{m}$ (D) and by $0.003(\mathrm{~m})$. $\quad D$ and $m$ have been expanded relative to table 3 so that each ranges to about four percent away from the optimum value. The viewing angle is described by THETA from the $Z$ axis and PHI from the $x$ axis.

\section{SUMMARY}

These trials using one micrometer polystyrene spheres demonstrate that the instrument provides a practical route towards aerosol characterization. $360^{\circ}$ scattering diagrams from individual aerosols in a flowing stream can be obtained at about $5^{\circ}$ resolution with noise sufficiently low so that both particle size and refractive index can be independently determined. Size can be established to within 0.5 percent and index of refraction to within about 0.7 percent. The data analys is by computer provides accurate statistical pictures of aerosols. Furthermore, particle sizing by $360^{\circ}$ scattering diagrams is not dependent on advance calibrations of the instrument as is required by many other methods.

The method offers some distinct advantages for aerosol spray characterization. Individual size measurements compile a statistical char- 
acterization of an aerosol with relatively high precision, and most importantly, without prior assumptions about size distributions. An accurate picture of real size distributions should emerge. This is particularly useful for measurements of aerosols which are rapidly aging. Refractive index measurements can be useful for studies of aerosols in which composition changes may occur during formation and aging. Solvent volatilization can be followed during aerosol aging, and component partitioning during aerosol formation can be recognized.

Extension to other size aerosols have not yet been completed, but initial efforts with $0.5 \mu \mathrm{m}$ particles show no barriers for that size range. Visual comparisons of calculated curves indicate that the present instrument will allow sizing of $0.3 \mu \mathrm{m}$ particles to within about two percent. On the other end of the scale, the apparatus can be set to size $20 \mu \mathrm{m}$ particles to within at least one percent, and it is probable that the range can be extended to somewhat larger particles without great difficulty.

\section{REFERENCES}

[1] Gucker, F. T. and Egan, J. J., J. Colloid Sci. 16, 68-84 (1961).

[2] Gucker, F. T. and Rowel1, R. L., Disc. Faraday Soc. 30, 185-191 (1960).

[3] Phillips, D. T., Wyatt, P. J., and Berkman, R. M., J. Colloid Interface Sci. 34, 159-162 (1970).

[4] Breuer, H., Gebhart, J., and Robock, K., Staub-Reinh. Luft 30, No. 10, pp. 25-31 (English Translation) (1970).

[5] Blau, H. H., McCleese, D. J., and Watson, D., Appl. Optics 9, 2522$2528(1970)$.

[6] Tuma, J. and Gucker, F. T., Proc. 2nd Int. Clean Air Congr., p. 463 (Academic Press, New York, N.Y., 1971).

[7] Gucker, F. T., Toma, J., Lin, H.-M., Huang, C.-M., Ems, S. C., and Marsha11, T. R., J. Aerosol Sci. 4, 389 (1973). 
MILTON KERKER: I noticed that your polystyrene distribution was negatively skewed. It was asymmetric with relatively more particles of the smaller size than you get from a Gaussian distribution. We found the same thing. This is opposite to what people normally accept as a natural distribution, i.e., a positively skewed logarithmic distribution. It will be interesting to see how that holds up under further work and whether the asymmetry has some statistical information to impart concerning the formation of these particles.

PARMENTER: We would, of course, feel much more secure had we measured as many particles as you did. However, these results are from about 100 particles, so I'm sure the trend is correct. I've no reason to believe that this distribution is in error.

KERKER: The other thing is that we also tried to fit the size with the refractive index, and I would have to go back and look at our data again. I think they were within the same ballpark. We did not consider this a successful way of getting the refractive index. That is, we found also that if you perturb the refractive index by about .01, you got a size that was different by an amount greater than you would normally be willing to accept. This is something, of course, that would be interesting to pursue.

PARMENTER: We are finding the opposite now. We feel that generally the computer can make a distinct choice of combinations.

KERKER: We were strictly using visual estimates. One could use a method of steepest descent or something to come into the well and find out where you are, but we really didn't do anything like that. We just kind of eyeballed these ....

PARMENTER: We were unable to do this by eye alone. The index of refraction is more difficult to see by eyeballing, so your experience is consistent with ours.

KERKER: It's worth looking at more precisely. Finally, what criterion did you use as a measure of fit between experimental and computer results? We just used the least squares type.

PARMENTER: That is exactly what we are using.

ROBERT KNOLLENBERG: It appears that once your particle size is as you say up to about 20 microns, you have several more points to fit in your set-up. Is this not correct? It appears that you have a problem in resolution if you are to acquire all the data counts in 20 milliseconds.

PARMENTER: We have $5^{\circ}$ resolution in the optics, and the resolution on the data acquisition is about $1^{\circ}$. 
KNOLLENBERG: It is true your number of data goes up as your particle size increases?

PARMENTER: It would have to go up as the particle size increases. That's clear.

EDGAR ETZ: When you do these computer fits, I believe you said you analyze the sum of the squares of the deviations of the experimental values from the calculated curves. You must have an estimate of the uncertainty in the experimental scattering intensities because you might come up with an equation that fits the experimental curve much better than is actually warranted.

PARMENTER: We fit the noise in other words?

ETZ: Yes. Consequently, you get so many different solutions--some are real and some are not real. You come up with more than one minimal value in this standard deviation.

PARMENTER: We haven't yet made a careful analysis of it. I might say that we have worried about it and made a preliminary exploration of it in the following way. You saw the four curves which came from the same particle. That represents very typical noise--at least in this particle range. We let the computer look at each one of those curves individually to tell us what the particle was. The computer decided that the particle was identical in three of those cases and in the fourth case the computer decided that the particle was different in size by $5 / 1000$ of a micron--about 0.4 percent which is a neighboring size increment. So this indicates that noise is not going to introduce a serious error in this analysis.

GREG ROSASCO: You said you used a logarithmic amplifier? Would pulse counting techniques and then a computer conversion to logarithmic output help you in your sensitivity?

PARMENTER: I think it probably would. We are in the process now of considering how to build a better instrument, and this is one of the things we have to consider. The problem that we would encounter with pulse counting techniques is pulse pile-up. I don't know if this is going to be severe or not. We haven't yet gone through it carefully. It would be great if it works because that would eliminate several other problems.

SINNOTT: For about $\$ 500$ you can buy a phototube that has a two microsecond pulse pair resolving time. 
NATIONAL BUREAU OF STANDARDS SPECIAL PUBLICATION 412

Aerosol Meosurements, Proceedings of o Seminar on Aerosol Measurements

Held at NBS, Goithersburg, MD, Moy 7, 1974 (Issued October 1974).

\title{
ACTIVE SCATTERING AEROSOL SPECTROMETRY
}

\author{
Robert G. Knollenberg \\ Particle Measuring Systems, Inc. \\ 5469 Western Avenue \\ Boulder, CO 80301
}

\section{ABSTRACT}

An active scattering aerosol spectrometer is one that uses the active open cavity of a laser as the source of particle illumination. The interferometric aspects of the oscillating radiation illuminating the particles produces both forward and backward scattered radiation at all collecting angles. This, coupled with the fact that the collecting optics solid angle can be considerably greater than one steradian, and an extremely intense source, results in a system with an extremely high sensitivity, fully capable of sizing particles several hundred Angstroms diameter using solid state silicon detectors.

Key words: aerosol light scattering; aerosol sizing; aerosol spectrometer; cloud droplet measurements; interferometer; laser imaging of particles; laser light scattering by aerosols; particle size measurements.

I've decided to change the content of my presentation considerably in light of the presence of the FDA and their interest in aerosol can products, but I will cover the active scattering spectrometry at the end of the presentation. What I'm going to do is outline several techniques that we use to design instruments which we build for various customers. These range from imaging systems to single particle extinction. With particle scattering we have two types of techniques: one we call classical scattering and the other we call active scattering.

Let me mention briefly that the type of imaging system we use essentially involves a collimated light field where we project the shadows of particles and onto a photodetector array (fig. 1). At one time we did use fiber optics with photomultiplier tubes; today we use photodiode arrays. This type of system is one that we designed for use in sizing droplets from aircraft and has a size range at aircraft speeds of about 5 to 5,000 micrometers. Not within one instrument mind you, the instrument itself typically has 32 elements so that we can resolve one part in 32. The system has been designed in two standard probes, one covering 10 to 320 micrometers and a larger probe that covers about 200 to 4500 micrometers. I'm going to pass around some photographs so that 


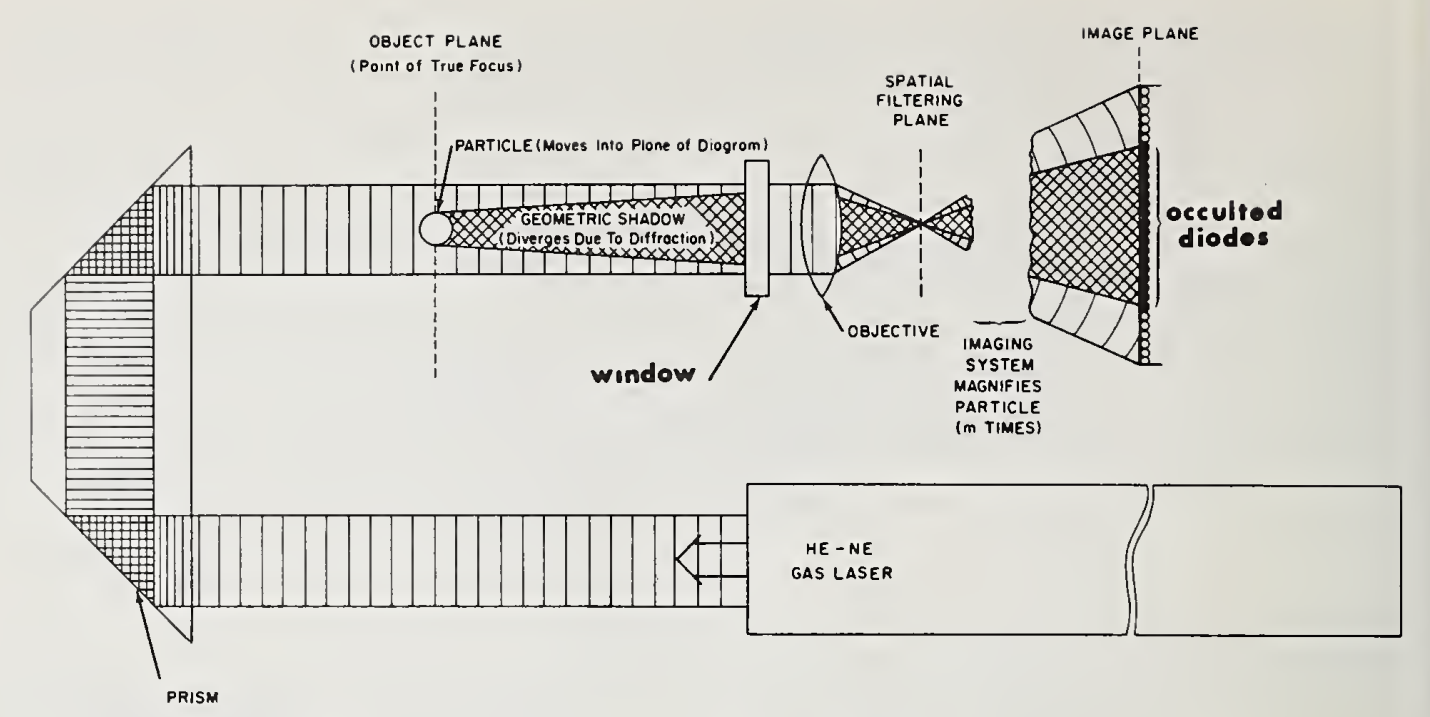

Figure 1. Schematic of imaging system designed to size droplets with airborne equipment.

you can see what the instrument looks like (fig. 2). These instruments have been mounted and used on 17 different aircraft.

I would say that even in the case of aerosol particles if we could use imaging techniques we would. In fact, one of the things we looked at for the Johnson Space Center recently was a possibility of extending

Figure 2. Photograph of imaging system instrument used on aircraft. 
an imaging system such as this down to submicrometer particles. The question is what we do about the fundamental limitations. What we chose to do was to accept working in a vacuum, and to use a CRT gun and focus it down to an electron beam on the order of one micrometer dimension. We can keep it collimated to a field of something like $5 \mathrm{~mm}$. We then diverge the beam much in the same way the lens refocuses the particles. This system is one that we have proposed, but has never been built. It would appear to have the merits of an imaging system and yet have the resolution on the order of 0.1 micrometer.

One might say that this would not have applications in the area of, let's say, atmospheric pressure. This really isn't true because you can operate the CRT gun in a scanning mode as you would with a scanning electron microscope. If you have ever used one you notice you can in fact light the thing up at subatmospheric pressure. If you $A C$ couple you can separate out the signal. The limitation actually comes through the bandwidth limitation produced by the scan of the particles. Now a tenth of a micrometer at one meter per second gives you a transit time of 0.1 microsecond, so right now the problem appears to be stymied because of the problem of scan times in the CRT. This is a technique that you could use to image down to around 0.1 of a micrometer if one had the capability of slowing down the particles. Given this, an electron beam equivalent technique that could be used for larger particles at an optical wavelength.

Below 5 micrometers it turns out that we have two types of systems to choose from; both extinction and scattering. In extinction system one typically focuses a laser beam down with an f/l00 lens to something around 100 micrometers in diameter. Actually, one can go smaller to 30 micrometers diameter. You can look at the cross section of the beam and match that with the extinction cross section. If you calculate what you have to have in signal-to-noise ratio to resolve it, it's not easy. If you want to resolve 1 micrometer with a 100 micrometer beam diameter your signal to noise requirement is 10,000:1. In addition to that being almost impossible, you have to have a large dynamic range. But we have set up extinction systems to resolve down to as low as 1 micrometer with a 30 micrometer diameter beam which lowers this requirement to $10^{3}$ or more. This can be used. The advantage of going to extinction is that extinction cross sections are a little better behaved, let's say, than what our scattering cross section is, and of course the difference is that we are not quite as refractive index sensitive. We do not commercially build this kind of instrument. We have built two but they have been for special applications where the source concentrations have been very high.

In terms of classical scattering systems, there is a technique using a focused laser beam. The beam is dumped on a spot on a lens and a light is collected by the detector. The scattering light from the particle will typically pass through in this direction. With this kind of a system we have operated at aircraft speeds with a resolution of 0.5 micrometers. This instrument is refractive index sensitive. It is designed for water droplets for which the refractive index is known, and 
we calibrate typically with glass beads and then shift a calibration curve. If you use it in the range of collecting angles from something like 7 to $20^{\circ}$ you can get a monotonic calibration curve.

The last technique I wanted to describe which was proposed as the subject of discussion here is the active scattering technique. This technique is one we have been working on for a couple of years. It involves the use of an open cavity laser (fig. 3). Initially, one might say, what is the advantage of going to an open cavity laser? It turns out there are several advantages. Our initial intent was simply to gather more light. We found that several things actually happened. One is, of course, that if you use highly reflective mirrors on both ends of the laser you can achieve an energy density of somewhere between 400 to 600 times that which we get from putting in a standard dump mirror in the laser. The reason for this is that it typically takes 99 percent coupling internally for a helium neon laser to even lase. So you are using a one percent dump mirror. That means that at any time internal to the cavity there is probably 200 times the energy density that exists outside. Instead of using a 1 percent dump mirror, we use a 99,990 percent reflectivity mirror on both ends.

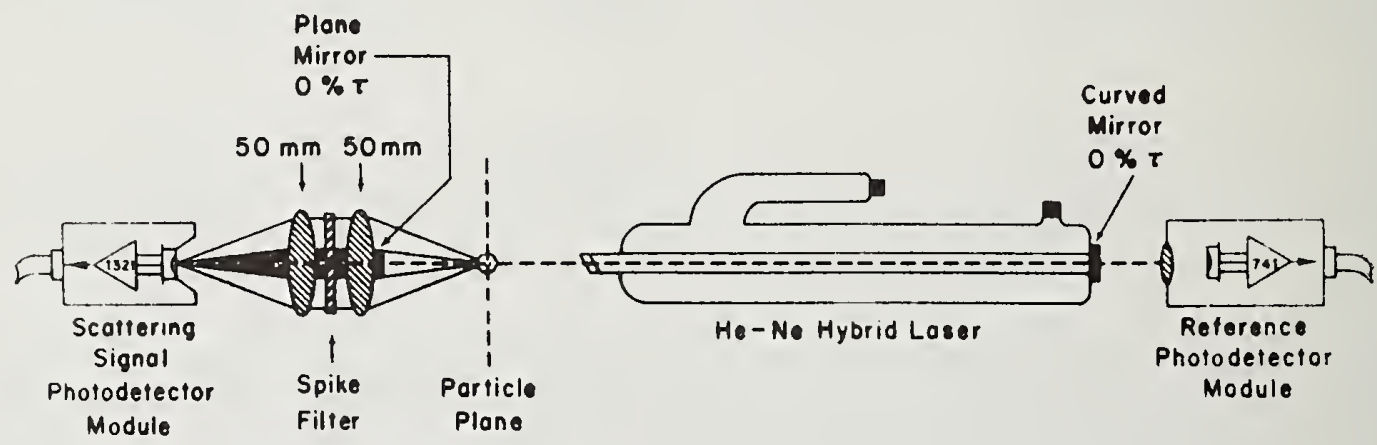

Note: Mirrors are reversed for large particle size ranges.

Figure 3. Schematic of active scattering technique system using an open cavity laser.

There are several things that happen here. We are trying in this case to collect light that has been scattered through angles from about 3 to $30^{\circ}$. It turns out that this is not a simple scattering case, but we are dealing with light that is actually traveling in both directions. So we also collect light that is being scattered from 177 to $150^{\circ}$. We have taken the classical Mie solutions over the collecting angles for the forward and backward components and superimposed these solutions. It turns out this is only the first approximation to the true solution which is solving the scatter equation in a standing wave which to my 
knowledge has not been done. We have set up our own program to solve the boundary conditions or changes in boundary conditions. We are about ready to work out new theoretical curves. We do know that it is not correct to simply superimpose the two solutions. I don't know why, but I suspect it's because the boundary conditions here are not quite the same. In any case in this kind of a system you typically collect 20 to 30 percent of the light scattering. Two other things I should say that are different. One is that we are dealing with about $10^{3}$ to $10^{4}$ watts per centimeter square. This is a very hot, very intense beam. Now you can reverse the cavity configuration. As I have it drawn here this is a flat mirror and this is a curved mirror. Now if I reverse them and put the flat here and the curved up here I can in turn change the dimensions of the beam, the size being typically something on the order of 200 to 300 micrometers at the flat mirror. I would like to point out two other things that happened. When we first started using this system, we noticed that the pulses coming out of our pulse height detector sometimes looked like this. Of course my engineers say it's oscillation, but what kind of oscillation is it? It turns out that what was happening was that particles would drift through at a slight angle. This is a built-in interferometer, so we get automatically out of the system a Doppler modulated pulse. We thought at first that this would be useful as a Doppler velocimeter as an intrinsic measure of velocity, so we could have a true measurement of flow rate through the beam. However, there is another point. The reason that the pulse is modulated, of course, is because the forward part of the Mie scatter in this region, here, is being heterodyned with the backscatter portion. As you know, once you get down to the Rayleigh particle, these two are equal in amplitude. Once the particle is very large, this one is very strong. So the modulation depth is also a measure of size. It also turns out that we have done some studies and the number of waves across the pulse is a function independent of the speed. It has to do with path length through the beam. Why is that important? Typically, unless we run a high order mode we are dealing with a Gaussian beam, we would like to know where particles go through the beam without having to put jets into the system. We've developed circuits that can do this on a pulse width rejection basis. However, it appears now that we can actually have this feature here as well. I would point out that simple logical steps remain.

We don't right now use the modulation depth as a size measurement, we simply use pulse height measurements. It is a near forward scatter system and it is also a near backward scatter system with a high collection efficiency. Our typical sensitivity in terms of converted photocurrent is one microamp of signal per micrometer squared of scattering cross section. Anyone knows that if you run that through a one megohm resistor you have a one volt pulse. Now what it means basically is that we do not use, in this kind of a system, photomultiplier tubes; we use solid state detectors. It gets around the familiar problem that we've always had with drift associated with photocathode sensitivity, particularly when it's hit hard, and this is a lot of current for any photocathode. So we have a system in which we collect scattered light using 
a photodiode. At the moment there are two limitations to this system. If you were to use an Argon laser you could go up another factor of 100 or even 1,000 in intensity. Calculations show that at 500 Angstroms, a particle with unit adsorbtivity and a heat of vaporization equal to that of water will evaporate in that beam, so you cannot arbitrarily increase the energy density at will. Another limitation, is due to the molecular scattering from a gas. This gives rise to a large background noise component with which you must compare your particle scattering intensity. So with these two limitations in mind, we usually cut off any system of this type around 500 Angstroms. It is a highly sensitive system, and gives us sensitivity simply by providing a very intense beam.

This system actually was conceived not for routine aerosol measurements, but for measurements in a vacuum. Of course it has certain advantages in a vacuum. One is that we have designed optical systems that can locate that particle both in terms of depth of field and where it passes through the beam radially. We can design an extremely open system. In fact, we have designed a system with an open area of some 6 square inches. This photograph here shows a system with two heads that was used in tests at the Marshall Space Flight Center. That system had a sensitivity of about 0.1 micrometer. Actually, it had two size ranges, 0.1 to 1.5 and 1 to 29 .

The other thing I would like to point out is that because this is a collimated system, you can, within the limitations of the optics, obtain a depth of field of something like $\pm 5 \mathrm{~mm}$ without an appreciable change within your scatter function. In an ordinary system where you have a highly focused illumination, you go through quite a change in intensity, so you have a very small path in which you allow particles to interact. It's not true in this case. You can accept information over quite a path length.

One other thing I wanted to point out is that we call the probes optical array spectrometers. If you take each one of those diode elements, connect it with its own data storage register, that is a shift register, you can achieve a 2-D imaging system. We've done that now and I'1] just pass around some images of particles that we've taken with a 2-D spectrometer hooked up in that fashion. Some of these you'll see have bright spots in the center and that's an indication of transparency. We have designed that system primarily to determine crystalline structure in clouds, again for aircraft applications, and its limitation there is on the order of about 250 knots, and about 20 micrometers resolution. Again, in any imaging system (it makes no difference what kind) it has somewhere a bandwidth limitation that's tied to resolution. With moving particles, you only have so much time to resolve a certain linewidth. 


\section{DISCUSSION}

GEORGE SINNOTT: I wonder if the problem you refer to in calculating forward and backward is not simply that in this case the forward and backward waves have a phase relation to each other, so you can't simply add up the intensities?

KNOLLENBERG: No, we add the complex waves. We've made some progress. We've taken a slide and put a particle on it and looked at the scattering functions and they are not the same, not what you would calculate.

SINNOTT: And is not the modulation from the particle coming simply passing through the standing wave of the laser?

KNOLLENBERG: Yes, that's Doppler shift. You have to have some angle into the axis of the beam. This would be hard to measure with small cosine angles.

GEOFFREY HOTHAM: You mentioned the very intense beam inside the cavity. Can you measure aerosols with it without them vaporizing?

KNOLLENBERG: Oh yes. The reason the 500 Angstroms is a cut-off is because your surface to mass ratio goes as $1 / r$ so the problem becomes more severe the smaller the particle.

HOTHAM: How small can you go?

KNOLLENBERG: We would predict by this that you would get into trouble somewhere below 500 Angstroms. This turns out to be a limitation you would place on any kind of system that was that intense.

ILAN CHABAY: Is there any chance of you getting any radiation pressure in terms of motion on a particle beamed at that kind of energy density?

KNOLLENBERG: That's possible.

CHABAY: This question is related in some sense. I wasn't quite clear that I understand your statement about the heterodyning. Are you expecting to get information on particle size from the heterodyning?

KNOLLENBERG: It would appear that for some submicrometer particles, that you can select the near forward scatter and let's say back where the intensity peaks out at $180^{\circ}$. If you would ratio these two then you would have a measurement of some function of size. The modulation depth of the beam is exactly this ratio. In other words, for small particles this proportion rises if you have a higher modulation index. 
CHABAY: But you don't get that actually. That arises simply from the amplitude of the ratio of the forward and backward Mie amplitudes rather than from the size of the Doppler shift itself, that is not from the motion of the particle.

KNOLLENBERG: That's right. The amplitude is a function only of size. I'm sorry the modulation index is a function only of size. going across.

BILL YANTA: The spacing there is just due to velocity as it's

CHABAY: That was what I was trying to find out. Whether the spacing or the depth itself is useful in getting the information.

KNOLLENBERG: The frequency, let's say, gives you velocity. If you have particles that are very fast, you'l1 have a higher frequency. The only thing I said was that the number of waves across the beam is independent of velocity. So you can in a sense measure the beam width for each particle. 
NATIONAL BUREAU OF STANDARDS SPECIAL PUBLICATION 412

Aerosol Measurements, Proceedings of a Seminar on Aerosol Measurements

Held at NBS, Gaithersburg, MD, May 7, 1974 (Issued October 1974).

\title{
RAPID MEASUREMENT OF DROPLET SIZE DISTRIBUTIONS \\ BY OPTICAL HETERODYNE SPECTROSCOPY
}

\author{
Ilan Chabay \\ Department of Chemistry \\ University of Illinois \\ Urbana, IL 61801
}

\section{ABSTRACT}

A new technique which allows rapid, direct determination of particle size distributions by measurement of the Doppler shift of laser light scattered by falling particles will be discussed. The technique has been successfully applied to the measurement of parameters associated with cloud droplet growth (from 1 to 10 micrometer radius) in a diffusion cloud chamber. Applications to other types of particles in the size range 0.5-50 micrometers radius will be pointed out.

Key words: aerosol cloud chamber; aerosol light scattering; aerosol size measurements; aerosol spectrometer; cloud droplet measurements; Doppler measurements of particle size; laser heterodyne; laser scattering by aerosol particles; particle sizing.

I will describe first the general technique of determining particle size distributions by optical heterodyne spectroscopy and outline the method of measuring the spectrum. Then I'll briefly mention some results obtained on cloud droplets which will serve as an illustrative example. Finally, I'll comment on the limitations of this method and what extensions to a broader range of problems in particle measurement are possible.

The experiment is performed by allowing the particles to fall through a laser beam which passes horizontally across a chamber containing the particles. The falling particles scatter light from the scattering volume which is defined and observed by apertures and a PMT. Scattering is observed at a small forward angle in the vertical plane. The frequency of the light scattered by the falling particles is Doppler shifted from the frequency of the incident light. The magnitude of this Doppler shift gives the instantaneous vertical velocity of the particles. These velocities in turn can be related to the particle sizes by means of the Stokes relation. Thus, the radij of the droplets passing through the beam is given by the Doppler shift frequencies, without regard to intensity.

The calculated Mie scattering intensity for a specific scattering angle and beam polarization provides the relative intensity value for 
each particle size. One can obtain the particle size distribution simply by dividing the Doppler shift spectrum point by point by the relative Mie scattering intensities. The particular power of this technique is that the size distribution can be obtained unambiguously without any a priori assumptions of its form by using simultaneously the information available on scattered intensity and on particle velocity at a single angle of observation.

Actual Doppler shifts that must be measured can be as small as a few parts in 1014. In order to achieve such high resolution, a stable laser with a minimum of low-frequency noise should be used. In the initial experiments on this technique, an $\mathrm{Ar}^{+}$ion laser was used. It was operated at $5145 \AA$ with about $100 \mathrm{~mW}$ power.

A part of the incident beam served as a local oscillator or reference signal after elastic scattering from walls and windows of the chamber into the collection apertures. The surface of the PMT acts as a mixer for the Doppler shifted radiation field and the unshifted reference field. Beat notes occur from mixing of the fields with different frequencies and these beat notes appear as amplitude fluctuations in the photocurrent. The photocurrent is the input to a real-time spectrum analyzer which is followed by a digital spectrum averager. The net result is that the information comes out as a plot of the power spectrum of the photocurrent.

The information term in the power spectrum is given by

$$
P_{i}^{\prime}(\omega)=i_{L O}<i_{S}\left(r_{\omega}\right)>N\left(r_{\omega}\right) r_{\omega}^{-1}
$$

The first term on the right hand side is the local oscillator photocurrent. Angular brackets around the second term, $i_{s}\left(r_{\omega}\right)$, indicate a timeaveraged quantity, which in this case is the photocurrent due to Mie scattering of a particle of radius $r$ (and which has an associated Doppler shift $\omega)$. The size distribution is given by the function, $N\left(r_{\omega}\right)$.

As an illustration of the technique, I would like to discuss very briefly some key results of our studies of cloud droplets $[1,2]$. This work was done in the laboratory of Professor W. H. Flygare at the University of Illinois and was done in collaboration with Professor Jerry Gollub of Haverford College.

A steady state, diffusion cloud chamber was used to produce the droplets under desired conditions. The scattering due to droplets falling through the horizontal laser beam was measured at $7^{\circ}$ downward in the forward direction. An example of the spectra obtained is shown in figure 1. These three spectra were taken about $0.5 \mathrm{~mm}$ apart in the $4 \mathrm{~cm}$ high chamber. Each spectrum is the averaged result of 128 scans which required about 20 seconds total. The dip in the spectra at $530 \mathrm{~Hz}$ is due to a minimum in the Mie scattering intensity which occurs for 


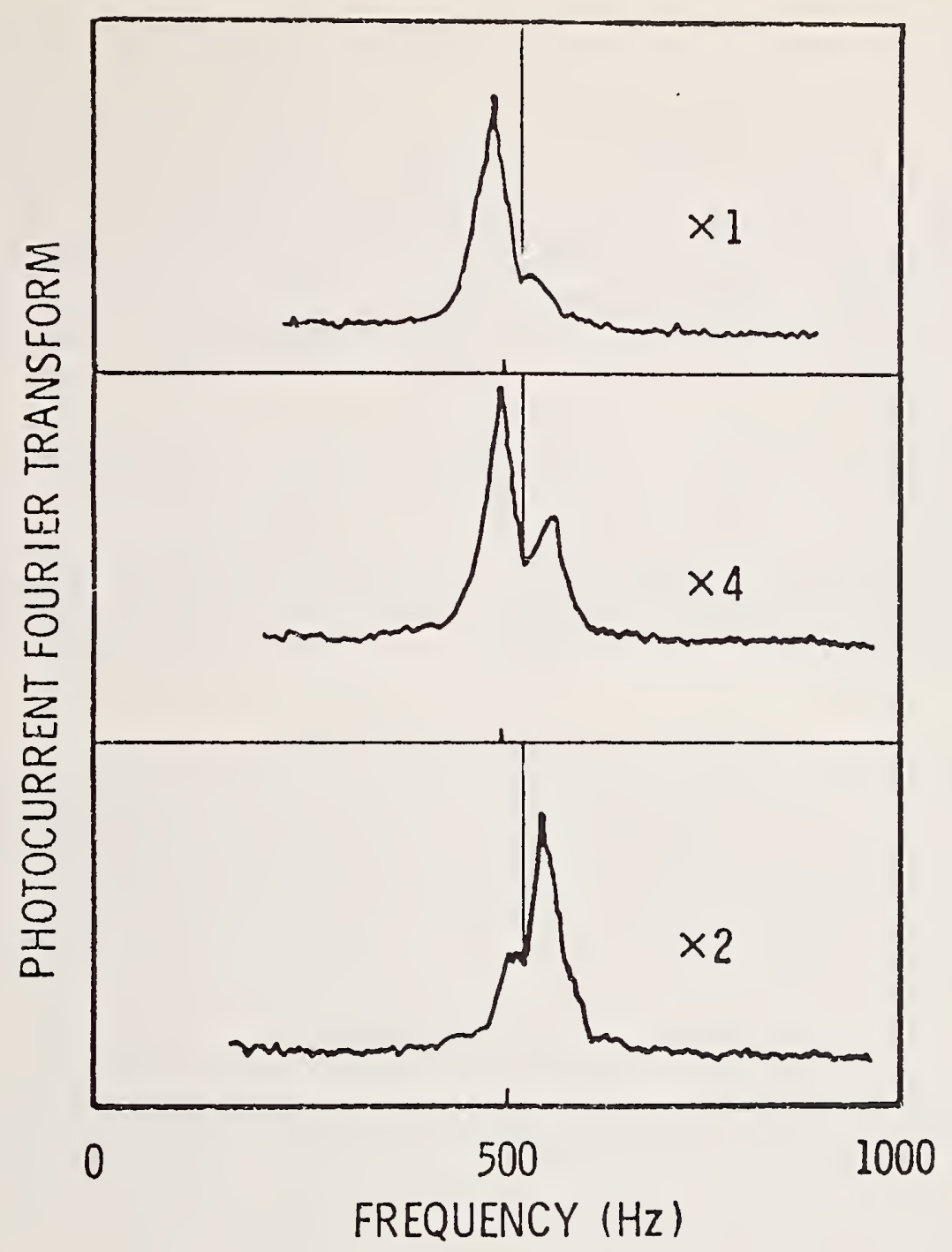

Figure 1. Aerosol spectra collected at three different heights in the cloud chamber.

particles of $4.24 \mu \mathrm{m}$ radius. The size distribution changed as different portions of the chamber were observed, but in the set of heights represented by the spectra shown, $4.24 \mu \mathrm{m}$ particles were present at each height. Therefore, the dip is present throughout and, of course, remained at the frequency characteristic of a particle of that size.

From spectra such as were just shown, the size distribution was easily found at any height in the chamber. The mean size as a function of height was then used to determine growth curves for the droplets as a function of conditions in the chamber. These growth curves then were compared to theories of droplet growth. 
The technique I've outlined here is directly applicable to particles with real or complex indices of refraction. The application is completely straightforward for particles which are spherical and range from 0.5 to $50 \mu \mathrm{m}$ in radius. This range can be extended to smaller particles by more complex (and less unambiguous) data analys is. The lower limit on size is due to the fact that the velocity due to gravity and that due to Browning motion become equal for particles of about 0.5 $\mu \mathrm{m}$ radius. Nonspherical particles can be treated by more complex calculation of the falling velocity as a function of size parameters and by calculation of the Mie scattering intensities for more complex geometries.

As I've indicated, this technique can be extended to a very broad range of particle sizes and materials. Information on particle size, size distribution, growth rates, and aggregation as a function of chemical and physical conditions can be readily obtained virtually in real time by this powerful technique.

\section{ACKNOWLEDGMENT}

Supported in part by the National Oceanic and Atmospheric Administrations Grant No. 04-4-022-10 to Haverford College and National Science Foundation Grant NSF-GH-33634 to the Materials Research Laboratory at the University of Illinois.

\section{REFERENCES}

[1] J. P. Gollub, I. Chabay, and W. H. Flygare, Applied Optics 12, 2838 (1973).

[2] J. P. Gollub, I. Chabay, and W. H. Flygare, submitted to J. Chem. Phys. 


\section{DISCUSSION}

MADHAV RANADE: Is that 20 seconds your measurement time or total?

CHABAY: Twenty seconds is the total time to accumulate 128 averages. A single scan and display of a spectrum requires $150 \mathrm{millisec-}$ onds. The minimum period of observation of the photocurrent depends on the frequency range, i.e., on the bandwidth of the spectrum analyzer.

RANADE: Isn't it true that within the time of observation you will have evaporation or growth of the water droplets in your cloud chamber?

CHABAY: Yes, but the point is that this is a steady state chamber. As the particles fall through the chamber, you have a steady state condition at each height. That is, if growth occurs through the chamber at known conditions of supersaturation as a function of height, the droplet size distribution is constant at each height.

MILTON KERKER: You pointed to the limitation that can be caused by the Brownian motion. What about convection? Isn't this a problem?

CHABAY: It's very definitely a problem. It's something that we struggled with in our first set of experiments. I think that it can be eliminated, certainly for particles of over a $1 / 2$ micrometer. I haven't tried to work with particles smaller than that. My guess is that it can be sufficiently minimized to allow work with even smaller particles, where one would determine a net diffusion constant, rather than the size distribution itself. One can measure the diffusion constant as an average quantity when Brownian motion is predominant over settling velocity. I think convection can be controlled well enough to do that by proper insulation of the chamber.

RONALD NELSON: How would you adapt this method to measurements of aerosol in aerosol cans?

CHABAY: The system is essentially the same. Basically one needs to have a very slow air flow into which the aerosol particles are introduced. A very simplified version would have a large scattering chamber with aerosol can on top. You push the button of the can and aerosol particles enter the chamber, fall through it, and are removed at the bottom. Let me show you the present apparatus (fig. 2). Water saturated air flows in through an annulus at the top next to the heated upper block, then down through the chamber to the exhaust annulus at the bottom. The wire screens help make the air and particle flow more uniform. If the entire chamber were kept at constant temperature and aerosol were sprayed in at the top, the same kind of data could be collected for aerosols as has been for water droplets. 


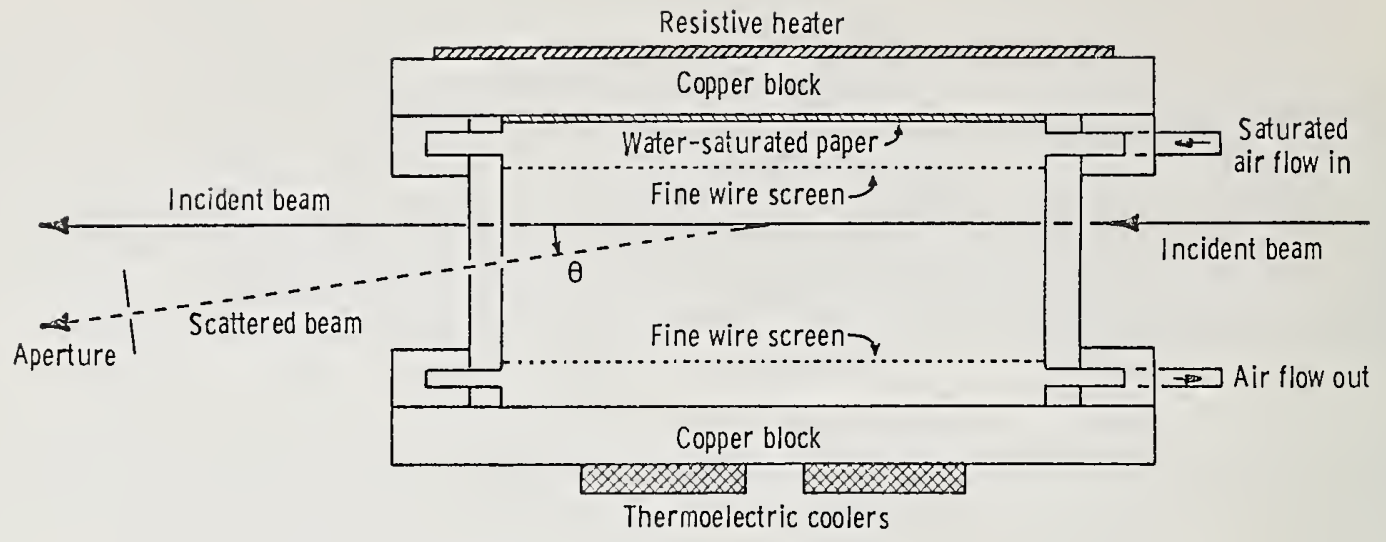

Figure 2. Schematic of cloud chamber for producing aerosols and proving scattering volume.

NELSON: Can you get absolute concentrations this way, with the essentially unknown flow-rate?

CHABAY: The flow-rate is something that one can determine independently but the absolute concentration is not something that we were trying to measure. We were really looking at the relative distribution of sizes of particles rather than absolute numbers.

KERKER: Are you familiar with Joe Katz's chamber?

CHABAY: Yes.

KERKER: Could this technique be used to get a finer handle on the rate of nucleation?

CHABAY: That was included in our original plans, but as yet we haven't had time to pursue it. One could change the chemical nature of the nuclei introduced with the inlet air, then observe the growth process as a function of the type of nuclei. This could complement the work described in a recent paper by Mirabel and Katz on binary homogeneous nucleation and aerosol formation.

KERKER: We are getting a little off the topic, of course, but are these people actually counting drops?

CHABAY: Right.

KERKER: But are you actually able to follow the rate of growth?

CHABAY: That's correct. 
KERKER: Howard Reiss of UCLA found that by using his cloud chamber and using the theory of nucleation by Byer and Greissers he could study kinetics of formation of droplets. What I'm thinking is that this heterodyne technique could give you a handle on growth rates.

CHABAY: I think so. We are just now publishing some growth curves from the data that we have collected, and I think that this could be extended to a very wide range of systems. It gives you very clearly the growth characteristics of the material.

KNOLLENBERG: In studying the nucleation of particles down to the order of 0.02 micrometer, you are talking about a nucleation technique with particles that would be limited to the order of one micrometer.

CHABAY: The point is that with this technique one cannot study directly the nucleation process for particles less than 1 micrometer, let's say. However, what one can see is the effect of growth as a function of the much smaller nuclei.

KERKER: Reiss is counting the total number of grown particles, and he is getting a rate of nucleation from that. He (Chabay) can go further back into the growth stage and actually follow the growth--not all the way back, no.

CHABAY: No, I can only start at about $1 / 2$ micrometer size particle and work from there up to very large particles.

RANADE: They will have grown to a few micrometers in a matter of seconds.

CHABAY: You see the point is that in this kind of chamber the time scale for that actually to occur is irrelevant. I do not have to resolve the time scale, I only have to resolve the height. How well I can resolve a given time difference is a question of the size of the droplets, how fast they are falling, and how well I can manipulate the beam in terms of height--the resolution in height of this arrangement. I should mention in regard to this, that at the moment we were using a beam of about $2 \mathrm{~mm}$ diameter as a matter of convenience. We just used the output beam directly. One could focus that down to a much smaller beam, and get a higher resolution of the distribution and also greater detail in terms of the growth process.

CARY GRAVATT: Do you get instant readout on the particle distribution and how long does it take?

CHABAY: What you get is the spectra such as I showed you, which are power spectra. In order to get a size distribution out of that, you have to divide by the Mie scattering curve which is a fairly trivial arithmetic operation. A better way to do it, which we didn't do because we didn't have facilities to do it directly, but I hope to do it while I'm here at the Bureau, is to do processing directly on-line with the 
computer. That is, one could do this whole thing on the computer and immediately process the data so it would be a matter of fractions of a second after you have taken the number of scans desired that the size distribution would appear.

SINNOTT: Another technique very similar to this is to set up an interference pattern in the scattering region and then watch the particle scattered light fluctuations. that.

CHABAY: Right. I think Dr. Yanta is going to be talking about

SINNOTT: Do you have any remarks on the relationship of these two techniques?

CHABAY: Maybe it's appropriate to wait until Dr. Yanta's talk. I think generally the techniques are very similar. I know of no other study which has put together both the intensity and the falling velocity information to get the size distribution. You can certainly get the velocity of the droplet by the laser Doppler velocimeter measurements. The basic physics of the technique is the same. In our case, we do not image a real fringe field in space and look at the particle moving through the light and dark regions to determine the rate at which it goes through the fringes. The process of heterodyning gives the same information on velocity, though. 


\title{
MEASUREMENTS OF AEROSOL SIZE DISTRIBUTIONS WITH A
} LASER DOPPLER VELOCIMETER (LDV)*

\author{
William J. Yanta \\ Naval Ordnance Laboratory \\ Silver Spring, MD 20910
}

\section{ABSTRACT}

A miniature wind tunnel has been built which together with the Laser Doppler Velocimeter (LDV) has been used to determine aerosol size distributions. In principle the LDV was used to measure the particle lag of individual aerosol particles as they were accelerated through a small supersonic nozzle. The measured velocity lag was then used in conjunction with numerical predictions to determine the particle size. An optical owl was used to determine the mean of the size distributions. The LDV measurements were in good agreement with the owl measurements.

Key words: aerosol sizing; aerosol spectrometer; aerosol sprays; Doppler measurements of particle size; droplet sizing; interferometer; laser light scattering by aerosols; particle size measurements; particle velocity measurements.

\section{INTRODUCTION}

The Laser Doppler Velocimeter has been used for many years in measuring flow velocities. The primary requirement for the LDV is that micrometer size particles be present in the flow. The LDV then measures the velocity of these particles. If one assumes the particles are moving at the same velocity as the fluid (either gas or liquid), then the fluid velocity is inferred directly from the particle velocity. However, in air, particles greater than one micrometer may not follow the flow precisely, that is, the particle may lag the flow. This is especially true in supersonic flow [3]. It is demonstrated in reference 3 that the particle lag can be predicted if the particle size is known. Conversely, one can predict particle size if the particle lag is known. This particle lag can be determined from velocimeter results (particle speed) in a calibrated or known flow field from which the particle size can be deduced. The particle lag can be induced by placing a particle in a rapidly accelerating or decelerating flow field. A convenient method of generating this type of flow field is with a supersonic nozzle. Using the computational procedure described in reference 3 , one can readily predict a particle's velocity in a supersonic nozzle.

*This paper is a unification of references [1] and [2]. 


\section{THEORY}

A particle placed in an airstream will experience a force

$$
F=\frac{1}{2} \rho_{g}\left(U_{g}-V_{p}\right)^{2} A_{p} C_{D}
$$

where $\rho_{g}$ is the gas density; $A_{p}$, the cross-sectional area of the particle; ${ }^{V_{p}}$, the velocity of the particle; $U_{g}$, the velocity of the airstream; and $C_{D}$ the drag coefficient. If we assume: one-dimensional motion, a spherical particle and ignore potential force fields, then the force is given by the mass times the acceleration

$$
F=m \frac{d V_{p}}{d t}=\frac{1}{2} \rho_{g} A_{p}\left(U_{g}-V_{p}\right)^{2} C_{D}
$$

or

$$
v_{p} \frac{d V_{p}}{d x}=\frac{3}{4} \frac{\rho_{g}}{\rho_{p}} \frac{C_{D}}{D_{p}}\left(U_{g}-V_{p}\right)^{2}
$$

Thus, one can completely predict the particle behavior in a flow field by simply integrating the above ordinary differential equation. The ease of integration will depend on the complexity of the drag coefficient.

If one assumes the classical Stokes drag coefficient where

$$
C_{D}=\frac{24}{R_{S}}
$$

then a closed-form solution may be obtained. However, in order to take into account the effects of Mach number and Knudsen number, the drag coefficient given by the following equations [4] was used:

For $M \leq 0.5$

$$
C_{D}=\frac{C_{D_{0}}+\frac{51.1 M}{R_{S}}}{1+0.256 M\left(C_{D_{0}}+\frac{51.1 M}{R e_{S}}\right)}
$$


where

$$
\begin{aligned}
C_{D_{0}} & =\frac{24}{R e_{s}}+0.4+1.6 e^{-0.028 R e_{s}^{0.82}} \\
V & =\left|U_{g}-U_{g}\right| \\
M & =\frac{V}{\sqrt{\gamma R T}} \\
\operatorname{Re}_{s} & =\frac{V D_{p} \rho_{g}}{\mu_{g}}
\end{aligned}
$$

for $M>0.5$

$$
C_{D}=C_{D, C}+\left(C_{D, F M}-C_{D, C}\right) e^{-A R e_{S}^{n}}
$$

Where $C_{D}, C, C_{D}, F M, A$ and $n$ are given functions of $M$ in table 1 . The use of the above drag equation in eq (3) necessitates a numerical integration of eq (3). Thus, given the flow distribution through a particular nozzle configuration, one can calculate the particle's velocity along the nozzle. This can be done for a range of particle sizes. 
Table 1. Parameters in the drag coefficient expression.

$\begin{array}{ccccc}M & C_{D, C} & C_{D, F M} & A & n \\ 0.5 & 0.520 & 7.80 & 0.315 & 0.410 \\ 0.6 & 0.551 & 6.50 & 0.240 & 0.460 \\ 0.7 & 0.586 & 5.57 & 0.182 & 0.500 \\ 0.8 & 0.625 & 4.92 & 0.141 & 0.545 \\ 0.9 & 0.666 & 4.45 & 0.110 & 0.590 \\ 1.0 & 0.712 & 4.10 & 0.090 & 0.620 \\ 1.2 & 0.801 & 3.60 & 0.065 & 0.670 \\ 1.4 & 0.880 & 3.23 & 0.055 & 0.690 \\ 1.6 & 0.929 & 2.98 & 0.049 & 0.710 \\ 1.8 & 0.955 & 2.80 & 0.047 & 0.715 \\ 2.0 & 0.971 & 2.68 & 0.046 & 0.720 \\ 2.4 & 0.981 & 2.48 & 0.0455 & 0.725 \\ 2.8 & 0.969 & 2.36 & 0.0455 & 0.725 \\ 3.2 & 0.949 & 2.28 & 0.0453 & 0.730 \\ 4.0 & 0.919 & 2.17 & 0.0452 & 0.730 \\ 5.0 & 0.910 & 2.10 & 0.0451 & 0.735 \\ 6.0 & 0.910 & 2.05 & 0.0449 & 0.735 \\ 8.0 & 0.910 & 2.02 & 0.0448 & 0.740 \\ 10.0 & 0.910 & 2.00 & 0.0447 & 0.745 \\ 12.0 & 0.910 & 2.00 & 0.0447 & 0.745\end{array}$

\section{EXPERIMENT}

In order to determine the size distributions of aerosols an LDV electronic system was used in conjunction with a Mach 5 wind tunnel.

The optical arrangement used in this study is shown schematically in figure 1. This system is commonly referred to as the dual scatter or fringe system. The laser beam is split into two beams of equal intensity and then focused to a common volume (the measurement volume). At the location where the two beams intersect constructive and destructive interference takes place thus forming about 50 to 100 fringes (fig. 2). As particles pass through these fringes, the particles will alternately scatter and not scatter light depending on 


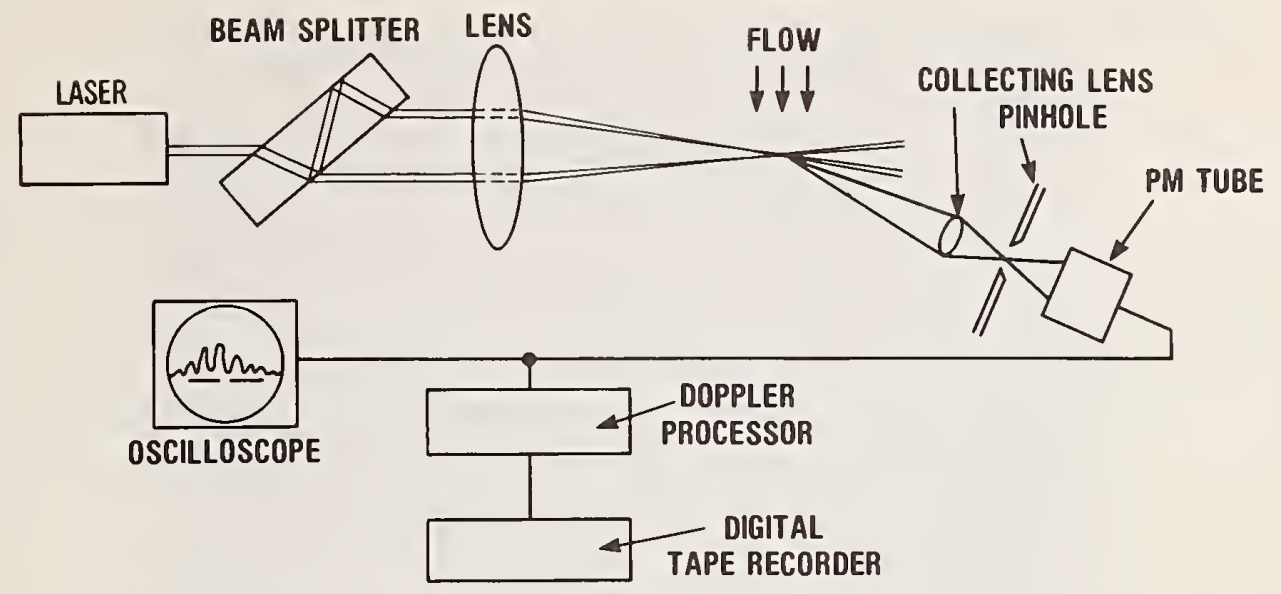

Figure 1. Schematic of laser Doppler velocimeter.

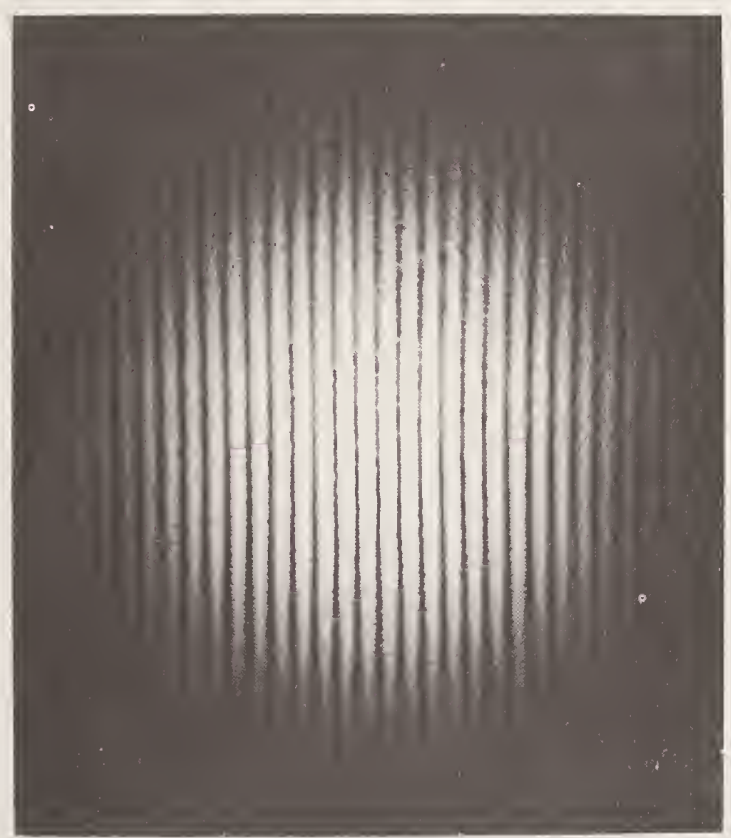

Figure 2. Optical fringe pattern.

whether the particle is in a constructive or destructive fringe. This scattered light is then detected by a photodetector. The output of the detector (as shown in fig. 3) is then electronically processed to determine the Doppler frequency. 


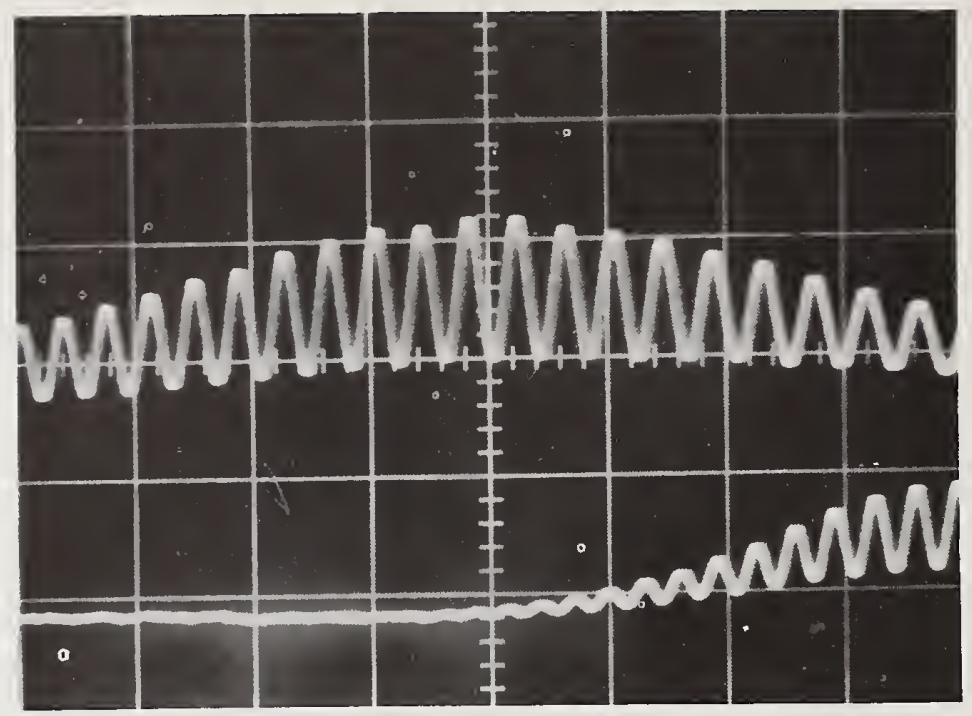

Figure 3. PM signal of particles crossing fringes.

The velocity $V$ can then be calculated with the usual Doppler equation

$$
V=\frac{\lambda f_{D}}{2 n_{0} \sin \left(\frac{\theta}{2}\right)}
$$

Thus, the velocity of an individual particle has been determined. Then by sampling a large number of velocities (on the order of 4000 samples) the size distribution may be determined.

The wind tunnel system is shown in figure 4 . The tunnel consisted of a gas supply (nitrogen), an aerosol source, nozzle, diffuser, dump tank and a vacuum pump. The supply pressure was maintained at a nominal one-atmosphere pressure. The general operation of the tunnel was as follows. The aerosol to be measured was placed in a storage tank. The ball valve was shut off, the vacuum pump started, and the rest of the system was evacuated to a pressure less than $1 \mathrm{mmHg}$. The valve was then opened, drawing the gas containing the aerosol through the tunnel. Measurements with the LDV were made 0.1 inch downstream of the nozzle exit.

With the system shown, the flow would "breakdown" after approximately 45 seconds or when the pressure in the dump tank was approximately $30 \mathrm{mmHg}$. This corresponds to a diffuser efficiency of about 50 percent. After a sufficient number of data points had been recorded, the ball valve was shut off and the system evacuated again. 


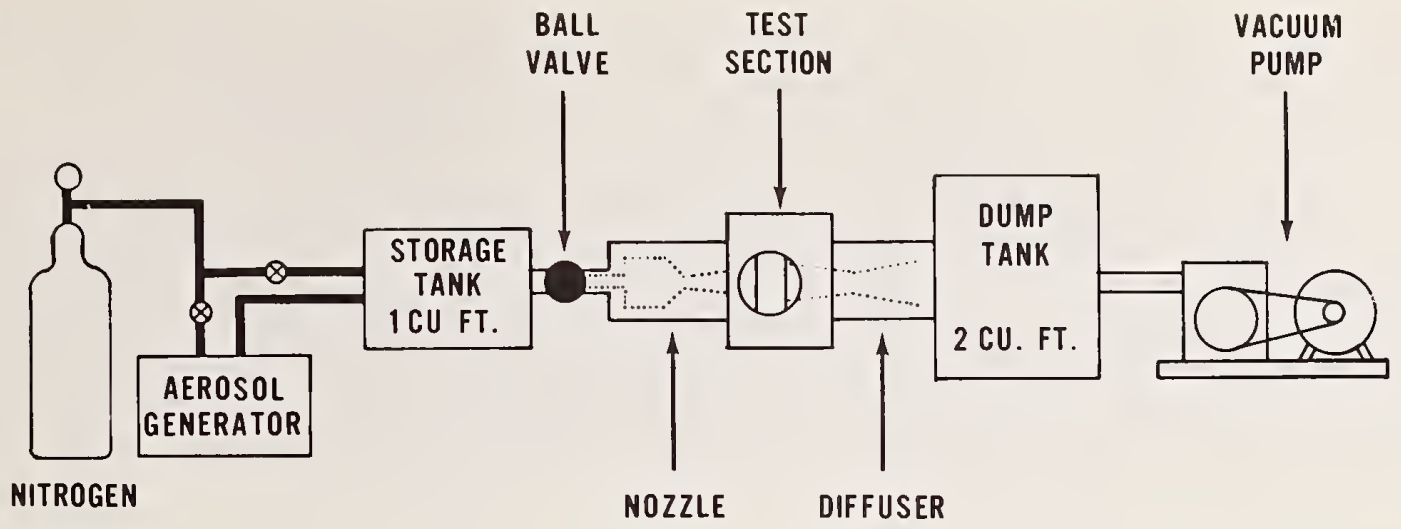

Figure 4. Schematic of miniature wind tunnel.

0il-pumped nitrogen was used as the working gas in place of room air for two discovered reasons. First, natural particulates were present in the room air and thus influenced the measured size distributions. The second reason was the presence of water vapor in the air. When the water vapor was expanded in the nozzle, heterogeneous nucleation takes place [5], thus forming ice crystals which also influenced the measured particle size distributions. Thus, in order to avoid these problems, oil-pumped nitrogen was used from a pressurized bottle. Since the mass flow through the tunnel was 0.0442 pounds/minute, no problem is encountered in maintaining this amount of flow from a single nitrogen bottle.

In figure 5 are seen the details of the conical nozzle and diffuser. The entrance angle of the nozzle was $45^{\circ}$ (half angle). The

\section{$-0.375=$}
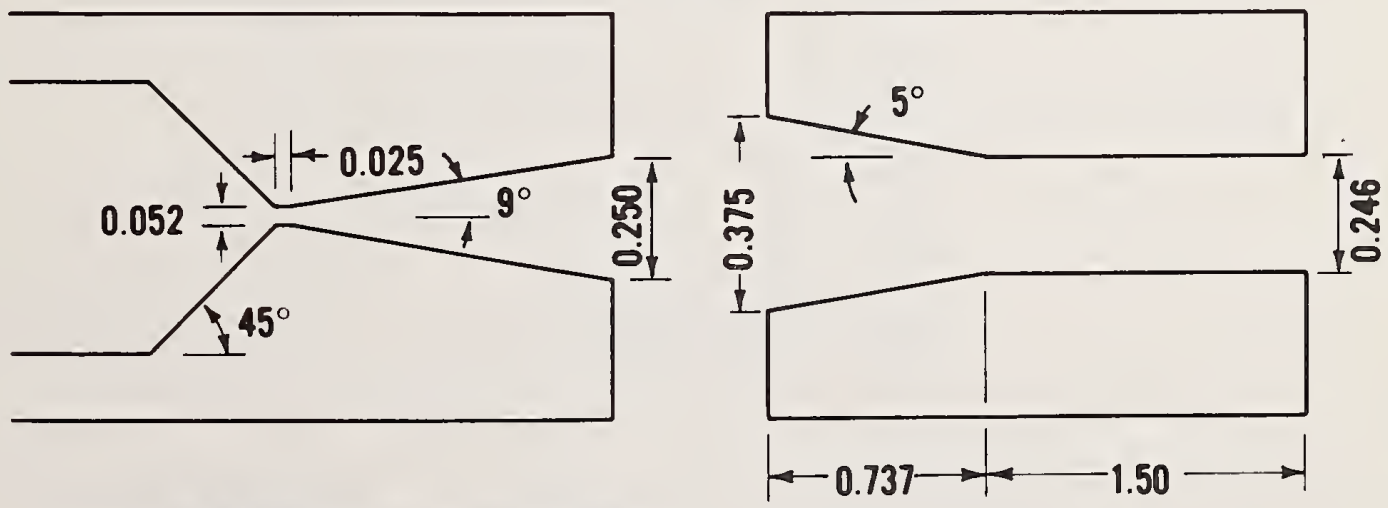

ALL DIMENSIONS IN INCHES

(NOT TO SCALE)

Figure 5. Details of nozzle and diffuser. 
throat was 0.052 inch diameter with a cylindrical throat length of 0.025 inch. The expansion half angle was $9^{\circ}$. The flow was expanded to Mach 5 at the exit of the nozzle where the area ratio, $A / A^{*}$, was 25 .

Following the principles outlined in reference 1 , the velocities for a range of particle sizes were computed at 0.100 inch downstream of the nozzle exit and these are shown in figure 6 . The computations were made for nitrogen having a supply temperature and supply pressure of $530^{\circ} \mathrm{K}$ and $15 \mathrm{psia}$, respectively. The aerosol material was dioctyl phthalate (DOP) having a specific gravity of 1.0 .

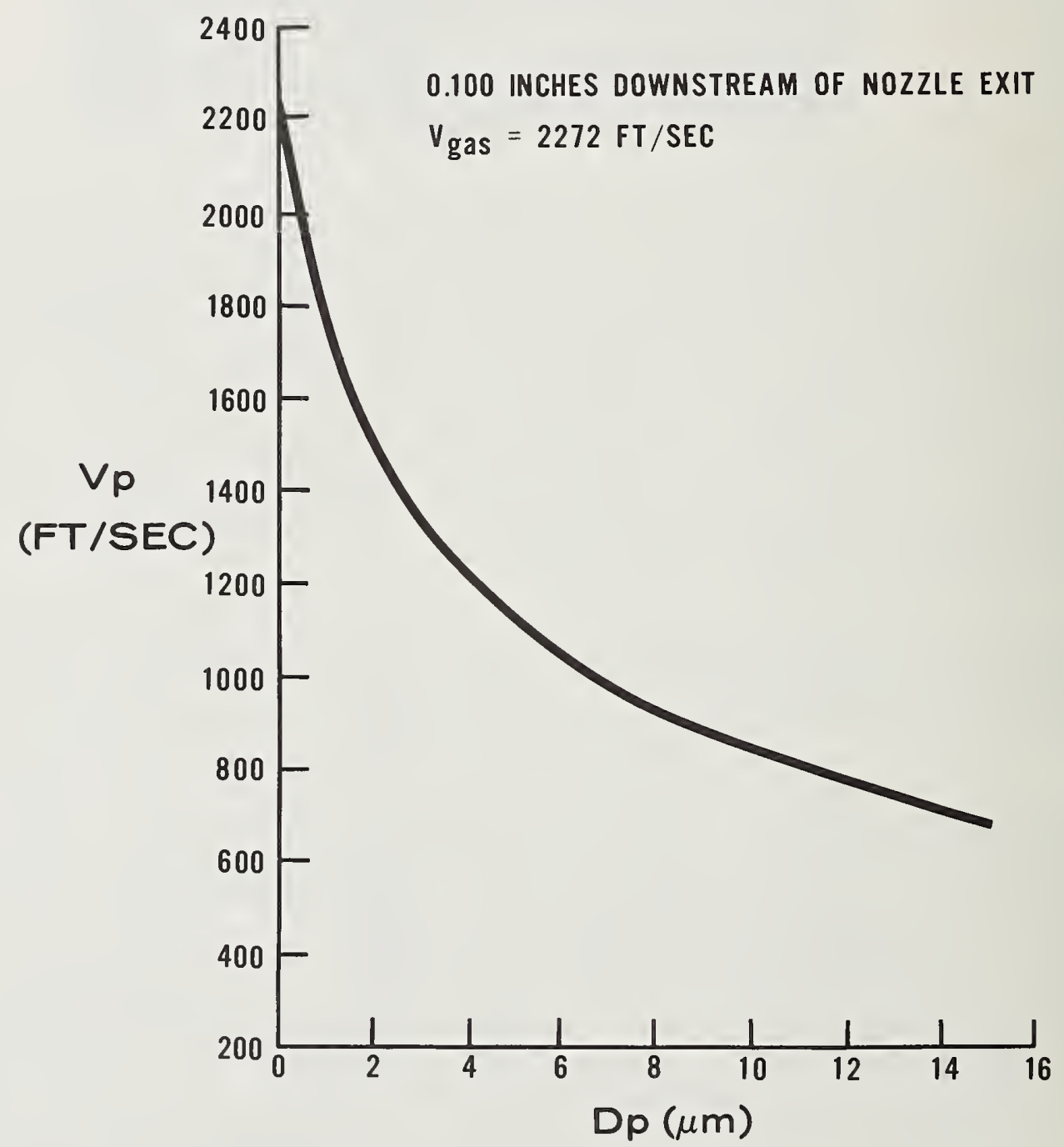

Figure 6. Particle lag at 0.100 inches downstream of nozzle. 
In order to generate particles having a suitable range of the mean diameter, the aerosol generator described in reference 6 and shown in figure 7 was used. The aerosol material is first atomized and the resulting polydispersed aerosol is vaporized by heating in an externally heated tube. The vapor upon condensation forms an aerosol which has a relatively narrow size distribution. The geometrical standard deviation, $\sigma_{\mathrm{g}}$, typically varies from 1.1 to 1.3 depending upon the mean particle size. This type of generator has an advantage in that the mean size can be decreased by using a solution of DOP diluted with a volatile

DRY COMPRESSED

AIR, 35 PSI

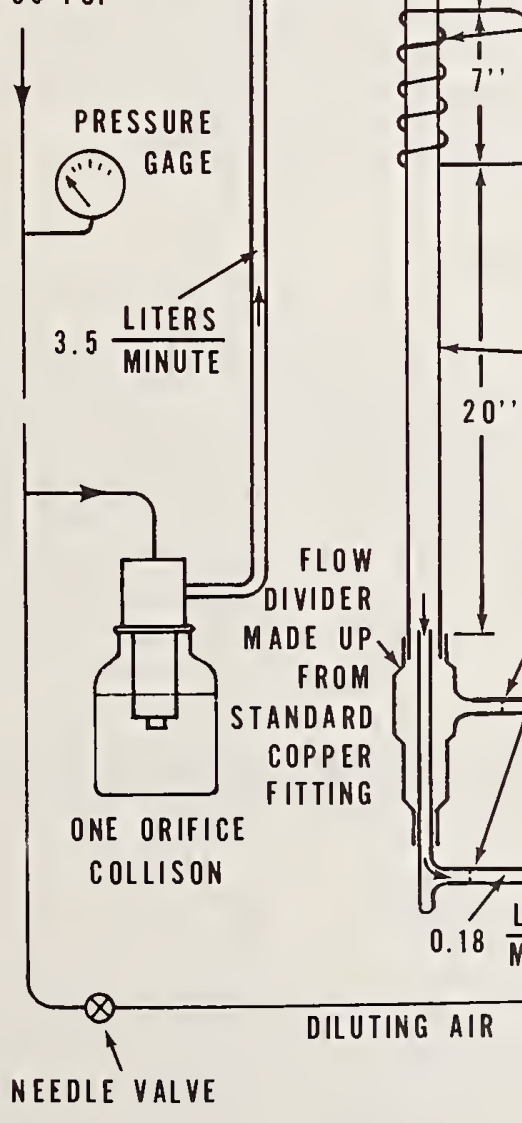

MADE UP F.ROM STANDARD COPPER FITTING

40 MESH SCREEN

HEATING TAPE, 1"'×2FT.

[192 WATTS WITH LINE VOLTAGE]
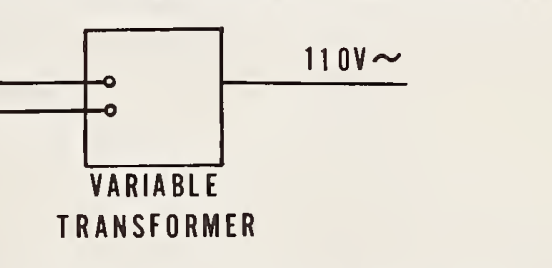

2 CM I.D. PYREX GLASS TUBE

NO. 47 DRILL HOLE

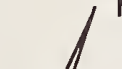

$3.32 \frac{\text { LITERS }}{\text { MINUTE }}$
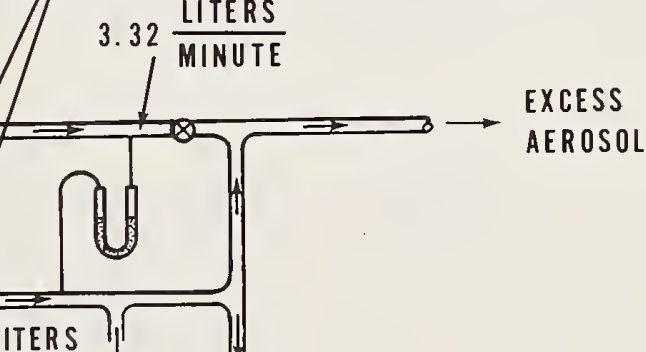

AEROSOL

Figure 7. Aerosol generator (Lui, Whitby, Yu). 
solvent. In the present tests, methyl alcohol was used. The diameter of the aerosol generated from the diluted solution is given by the relation

$$
D_{P}=D_{P_{0}}(C)^{1 / 3}
$$

where DPo is the diameter of the aerosol generated from 100 percent DOP and $C$ is the concentration of DOP in the diluted solution. This has been verified experimentaliy. For these tests four particle sizes were chosen, $\mathrm{D}_{\mathrm{Po}_{0}}, 0.75 \mathrm{D}_{\mathrm{Po}_{0}}, 0.50 \mathrm{D}_{\mathrm{Po}_{0}}$ and $0.25 \mathrm{D}_{\mathrm{Po}_{0}}$.

The optics is the same as used in reference 1. An argon 1aser with 1 watt of power at $5145 \AA$ was used. The optical volume was 0.015 inch in diameter and 0.085 inch long. The data was processed by a Doppler Data Processor fabricated by Arnold Research Organization, Inc. [7]. This type of processor is a burst-type counting system. The data from the processor was recorded on a high-speed incremental tape recorder and then processed on the Navy Ordinance Laboratory CDC 6400 computer. The recorder could record about 200 data points per second. About 4500 data points were used to determine each distribution. In order to obtain an independent calibration of the aerosol size, an optical "owT" described in reference [8] was used. The ow] is capable of determining only the mean diameter of an aerosol.

\section{RESULTS}

The results for the four velocity and aerosol size distributions in histogram form are shown in figure 8 . In order to obtain an accurate mean and geometrical standard deviation, the data is replotted in figure 9. The mean diameters obtained with the LDV and ow 7 are shown along with the standard deviation in table 2. It is seen that there is excellent agreement between the owl and the LDV. No diameter was determined with the owl for $0.25 \mathrm{D}_{\mathrm{Po}_{\mathrm{O}}}$.

Table 2. Comparison of LDV and OWL mean diameters.

$\begin{array}{cccc} & \text { LDV } & \text { OWL } & \\ & \text { MEAN } & \text { MEAN } & \sigma g \\ \mathrm{D}_{\text {Po }} & 1.30 \mu \mathrm{m} & 1.30 \mu \mathrm{m} & 1.15 \\ 0.75 \mathrm{D}_{\mathrm{Po}} & 1.10 \mu \mathrm{m} & 1.09 \mu \mathrm{m} & 1.23 \\ 0.50 \mathrm{D}_{\mathrm{Po}} & 0.71 \mu \mathrm{m} & 0.765 \mu \mathrm{m} & 1.14 \\ 0.25 \mathrm{D}_{\text {Po }} & 0.45 \mu \mathrm{m} & - & 1.22\end{array}$




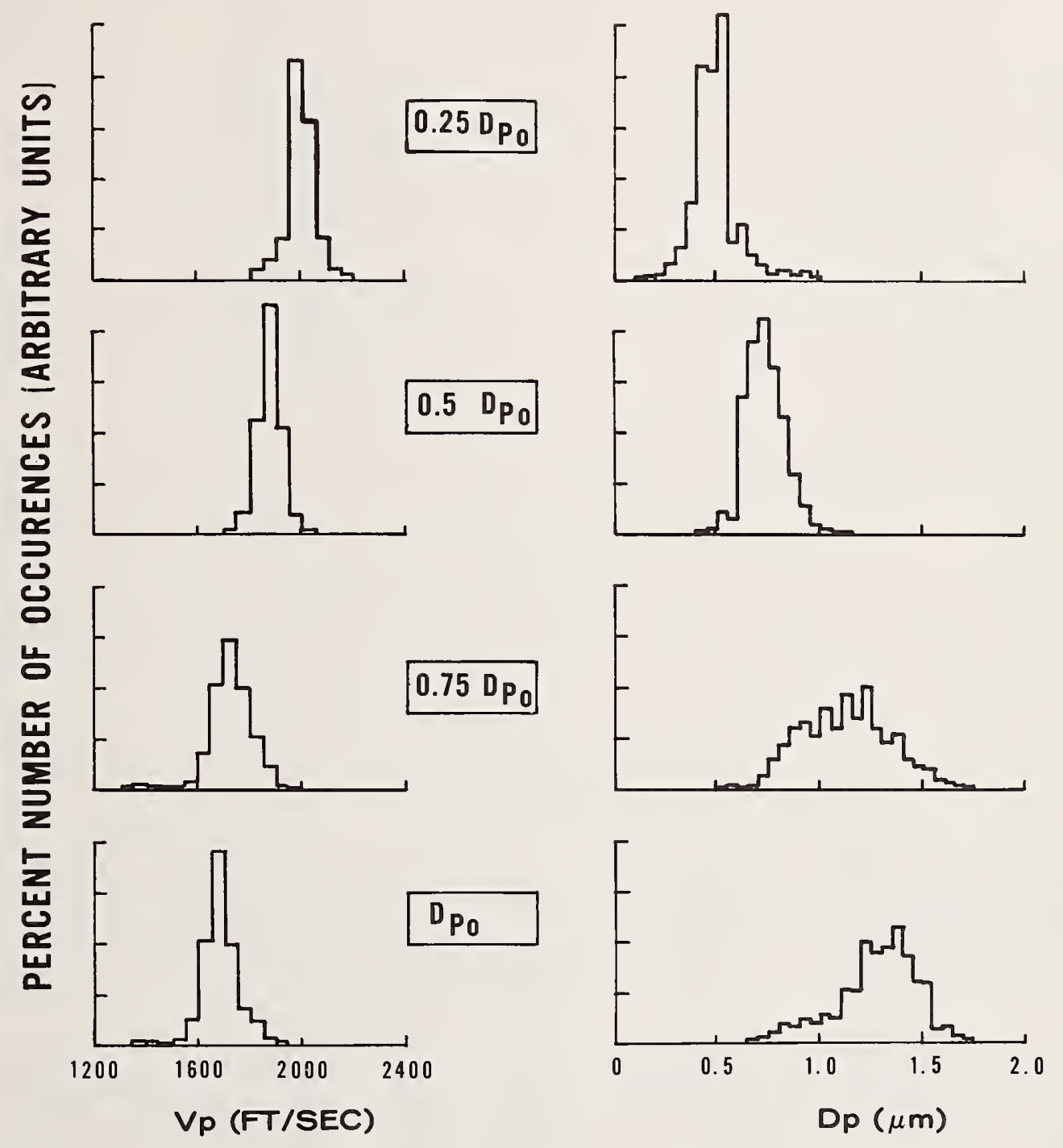

Figure 8. Particle velocity and particle size histograms. 


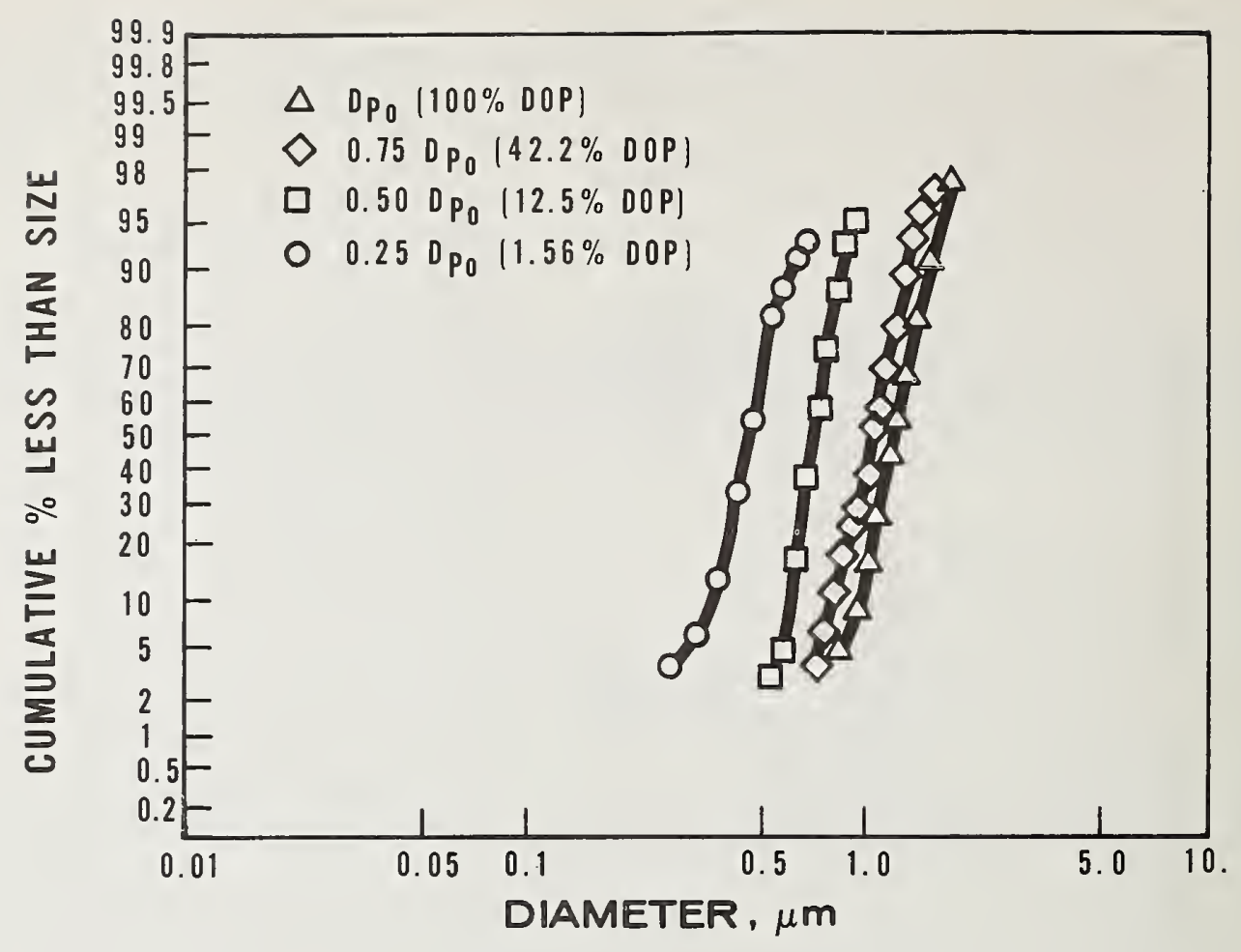

Figure 9. Aerosol size distributions.

\section{CONCLUSION}

A bench-type supersonic wind tunnel was designed, built and calibrated for use along with an LDV in determining aerosol size distributions. Excellent results were obtained between the LDV and the optical owl.

\section{NOMENCLATURE}

\begin{tabular}{|c|c|c|c|}
\hline A & constant in eq (6) & $n_{0}$ & index of refraction \\
\hline$A_{p}$ & $\begin{array}{l}\text { cross-sectional area of } \\
\text { particle }\end{array}$ & $\operatorname{Re}_{S}$ & $\begin{array}{l}\text { Reynolds number based on } \\
\text { particle diameter }\end{array}$ \\
\hline$C$ & concentration & $U_{g}$ & gas velocity \\
\hline & $\begin{array}{l}\text { drag coefficient of } \\
\text { particle }\end{array}$ & $v_{p}^{y}$ & particle velocity \\
\hline${ }^{C} D, C$ & $\begin{array}{l}\text { continuum drag } \\
\text { coefficient }\end{array}$ & $X$ & $\begin{array}{c}\text { distance downstream of } \\
\text { nozzle throat }\end{array}$ \\
\hline$C_{D, F M}$ & $\begin{array}{l}\text { free molecule drag } \\
\text { coefficient }\end{array}$ & $\theta$ & $\begin{array}{l}\text { angle between focused } \\
\text { laser beams }\end{array}$ \\
\hline & particle diameter & $\lambda_{0}$ & $\begin{array}{l}\text { wavelength of laser } \\
\text { radiation in a } \\
\text { vacuum }\end{array}$ \\
\hline $\begin{array}{l}D_{P o} \\
f_{D}\end{array}$ & $\begin{array}{l}\text { undiluted particle } \\
\text { Doppler frequency } \\
\text { Mach number }\end{array}$ & $\begin{array}{l}\mu g \\
\rho_{g}^{g} \\
\rho_{p}\end{array}$ & $\begin{array}{l}\text { dynamic viscosity of gas } \\
\text { density of gas } \\
\text { density of particle }\end{array}$ \\
\hline
\end{tabular}




\section{REFERENCES}

[1] Yanta, W. J., "Measurements of Aerosol Size Distributions With a Laser Doppler Velocimeter (LDV)," AIAA Paper 73-705, AIAA 6th Fluid and Plasma Dynamics Conference, Palm Springs, California, 16-18 July 1973.

[2] Yanta, W. J., "A Miniature Wind Tunnel For Use With the Laser Doppler Velocimeter in Determining Aerosol Size Distributions," Presented at International Short Course and Workshop on Laser Velocimetry, Purdue University, 25-29 March 1974.

[3] Yanta, W. J., Gates, D. F., Brown, F. W., "The Use of a Laser Doppler Velocimeter in Supersonic Flow," AIAA Paper 71-287, AIAA 6th Aerodynamic Testing Conference, Albuquerque, New Mexico, 10-12 March 1971.

[4] Cuddihy, W. F., Beckwith, I. E., Schroeder, L. C., "A Solution to the Problem of Communications Blackout of Hypersonic Re-entry Vehicles," Presented at the Anti-Missile Research Advisory Counci 1 Meeting, Annapolis, Marylanc, 22-24 October 1965.

[5] Stein, G. D., "Condensation of Ice Clusters by Homogeneous Nucleation from the Vapor Phase," Ph.D. Dissertation, Yale University, 1967.

[6] Liu, Y. H., Whitby, K. T., Yu, H. H. S., "A Condensation Aerosol Generator for Producing Monodispersed Aerosols in the Size Range, $0.036 \mu \mathrm{m}$ to $1.3 \mu \mathrm{m}$, "Journal De Recherches Atmospheriques, pp. 397406, 1966.

[7] Lennert, A. E., Smith, F. H., Kalb, H. T., "Application of Dual Scatter, Laser, Doppler Velocimeters for Wind Tunnel Measurements," International Congress on Instrumentation in Aerospace Simulation Facilities, IEEE-71-C-33 AES157, June 1971.

[8] Liu, Y. H., Marple, V. A., Yazdani, H., "Comparative Size Measurements of Monodisperse Liquid Aerosols by Electrical and Optical Methods," Environmental Science and Technology, Volume 3, Number 4, Apri1 1969, pp. 381-386.

\section{DISCUSSION}

ILAN CHABAY: By way of comparison to the laser doppler and the heterodyne techniques, the index of refraction does matter in the case that I'm talking about simply because you have to do the Mie scattering calculations for the distribution, but in your case you do not.

YANTA: It enters in indirectly because I could bias some particles if the index was such that they would not scatter much. 
CHABAY: But what I mean is that what you measure as the particles pass through is essentially the number of pulses that give you a signal rather than the intensity so you are not dependent on the Mie scatter.

YANTA: That's right. Once you reach a certain threshold level to make your electronics work, it doesn't make any difference if it's a half micrometer or a five micrometer particle as long as that signal is above a certain threshold for your electronics.

GEORGE SINNOTT: That's the difference between doing single particle work and scattering pattern analysis basically?

MILTON KERKER: He is using in place of a Mie calculation, a drag calculation.

YANTA: That's right. It's the drag equation, that's how I have determined the size of the particles. In other words, I've got to solve the equation of motion for the lag.

SINNOTT: I understand that, but take the particle in the interference region. Let's say you have 5 particles going through there at the same time at different velocities. How does your electronics handle that?

YANTA: There are several different ways. One is that when any particle enters--basically you take eight cycles of the data information and compute the frequency of the velocity. Now what happens if a particle either enters or leaves during that processing time, you get a phase shift in the signals and the electronics compares the first four cycles against the second four cycles. If those two times do not agree within a specific error usually like one and a half percent in the Doppler period, either we reject that data point or take the last one.

CHABAY: In our case the density is so high that there is a coincidence of particles where we get multiple scattering. Only if you get multiple scattering does it make a difference. If there are 4 particles falling at different rates, you see essentially 4 different Doppler shift frequencies and that's independent of ...

SINNOTT: You are taking a power structure. You see the difference. If you tried to do a Fourier transform of both particles at the same time going through there, by looking at both frequencies, then you have a problem of having a comparative intensity.

MADHAV RANADE: If you have particle sizes in the range of about 10 to 20 micrometers, the drag is very high. Does that effect the droplet chemistry and humidity?

YANTA: That's a good point. We are looking at that problem now. In the use of dioctyl-phthalate the problem did not seem to occur there. Now when we go into more volatile liquids with less viscosity and higher density, it could be a problem. 
KERKER: Is your illuminated region small enough so that you have to worry about velocity profiles in the gas stream itself?

YANTA: That's why I go off-axis with my collecting optics. Generally, my cross-beam volume is about an inch long, and my nozzle is only a quarter of an inch wide. By going off-axis I only look at a very small portion of that focal volume and the scan volume is 0.080 inch. There is no velocity profile, it is essentially flat across the exit.

RONALD NELSON: Do you have any trouble with condensation of particles within this extension, do you pick up water?

YANTA: A good point. When I ran this last week, we were just using room air and it was no problem. Last week a cold front went through the Washington area and the air was quite dry, but in the dead of summer when the water vapor is quite high it is a nuisance. I had to use a nitrogen source to make sure my gas was completely dried out.

NELSON: What is your sample flow-rate through this device? The Cc's of air or whatever?

YANTA: It's about $13 \quad 1 / 2$ cubic inches per second, but I'm only looking at a portion of that also. I'm not sampling the entire exit. There are about 4,000 samples in each one of those histograms. It takes about 20 to 30 seconds to obtain that. It is relatively slow because the slow part of the whole system was my tape recorder where I recorded my velocity data points. That's the slow part. The electronics is capable of around 5,000 per second, and we are thinking about buying a new set of electronics which is capable of a million data points per second.

ROBERT KNOLLENBERG: It's nice to be able to eliminate the necessity for something like that because one would like to operate at a reasonably high count rate, with lots of applications.

YANTA: I see no problem in going to something like at least 500,000 data points per second, and doing it in real time. Right now it goes through the NOL computer. I put the data on tape and process it on the computer, and I have some ideas of how to do it on-7ine. particle?

KNOLLENBERG: What is the dependence upon the density of the

YANTA: It is a direct linear dependence. The equations for the drag coefficients are very general in these calculations. What I've done - is to take a very elaborate set of drag coefficients, if you use the Stokes Law you can solve these equations in a closed form. However, inside the nozzle the static pressure is about $2 \mathrm{mmHg}$ and the Knudsen number is about 5, so we are starting to get into the problem of free molecular flow. The drag goes down considerably. 
JOHN WEINNEGER: How did you sample the room air freshener? Did you exit the spray into your holding tank? How much time after you put the spray into the holding tank if that's the way you did it, did you sample...

YANTA: The kind that we were using was about three or four inches in diameter, about 2 feet long with a volume such that we could effectively sample the entire tank in ten seconds. We pulsed the aerosol spray into the tank, so we made sure that there were some particles in the tank at all times. The maximum would be ten seconds in the inside of the tank.

WEINNEGER: How much time did it take you to get the histograms?

YANTA: About 30 seconds.

WEINNEGER: Total instrument time?

YANTA: Right. Total instrument time.

CHABAY: The LDV technique is similar to the optical heterodyne method. An important distinction is that the heterodyne method uses intensity and Doppler shift information (and requires knowledge of the index of refraction) to obtain a size distribution when any number of particles are present in the scattering volume. The only limitation is that multiple scattering be negligible. The LDV arrangement is independent of the index of refraction, but cannot deconvolute the intensity information if more than one particle is scattering at one time. 


\title{
AN OPTICAL TRANSFORM TECHNIQUE FOR MEASURING THE SIZE DISTRIBUTION OF PARTICLES IN FLUIDS*
}

\author{
Albert McSweeney \\ Experiment Station \\ George Institute of Technology \\ Atlanta, GA 30332
}

\section{ABSTRACT}

Real-time inversion of the data obtained from the total diffraction pattern due to particles in a relatively large volume yields a measure of the size distribution of the particles. A significant advantage of this technique is that the sample volume may be large, removing the requirement for piping the sample through small tubes. This technique may be applied to particles in gases, in liquids, or on microscope slides.

Key words: aerosol light scattering; aerosol sizing; aerosol spectrometer; laser light scattering by aerosols; optical transform; particle size measurements.

\section{INTRODUCTION}

The word "fluids" was used in the title of this paper to indicate that the technique to be described is applicable to the measurement of particle size distributions either in air or in liquids. Our first work in this area was centered on measuring the concentration of sediment suspended in water [1], and our present effort is directed toward the measurement of large particles in the atmosphere. The size range of interest is from 5 to 100 micrometers diameter.

\section{I . TRANSFORM TECHNIQUE}

The optical transform technique is based on the use of a coherent optical processing system [2] to measure the light intensity in the diffraction pattern due to a random group of particles. The light intensity as a function of radius is then inverted to yield a measure of the size distribution of the particles [3].

The optical system (fig. 1) consists of a laser to serve as an intense source of coherent radiation. Lens $L_{1}$ and Aperture $A_{1}$ constitute a pinhole spatial filter for removing undesired modes from the laser radiation pattern. Lens $L_{2}$ recollimates the filtered radiation, forming 

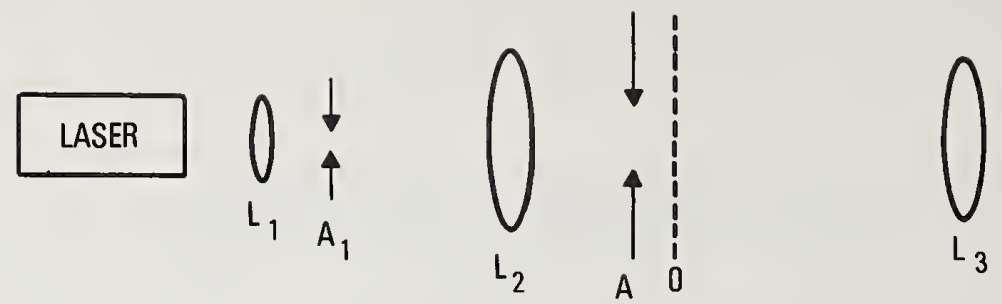

OPTICAL FOURIER TRANSFORM SYSTEM.

Figure 1. Schematic of the optical transform technique system.

a beam whose diameter may be several times larger than that of the original. Aperture $A_{2}$ limits the beam diameter and transmits the uniformly intense center section. The Fourier transform lens, $L_{3}$, has been corrected to yield diffraction patterns that are independent of the position of objects in the input region. Each particle in a group located in the laser beam will yield a diffraction pattern centered on the optic axis. The resultant intensity pattern is the linear superposition of the intensity patterns from all the particles provided the number of particles is large and their positions are random. Photographs of the optical system are shown in figure 2 .

For particles of circular cross section, the resultant diffraction pattern will be circularly symmetric. If the particles are of noncircular cross section, the diffraction pattern will still display circular symmetry provided there is a large number of particles and they are randomly oriented.

In either case the optimum detector for measuring the radial distribution of the light intensity is a detector that shows circular symmetry. Two types of circularly symmetric detector arrays have been used by the author. One is a commercially available photodiode array (fig. 3) and the other a meticulously arranged fiber-optic array [4] (fig. 4) which can be used with either photodiodes or photomultiplier tubes. A third arrangement utilizing a rotating mask with slits and a photomultiplier tube has been described in Applied Optics [5].

After the radial distribution of light intensity has been measured, it must be inverted to yield a measure of the particle size distribution. The inversion process has been reduced to a matrix multiplication in which a column matrix representing a set of light intensity data is multiplied by a rectangular inversion matrix to yield a column representing the particle size distribution. The inversion matrix may be derived from sets of light intensity measurements made on known monodisperse samples covering the size range of interest [3]. A comparison of the eigenvalues associated with an inversion matrix and 

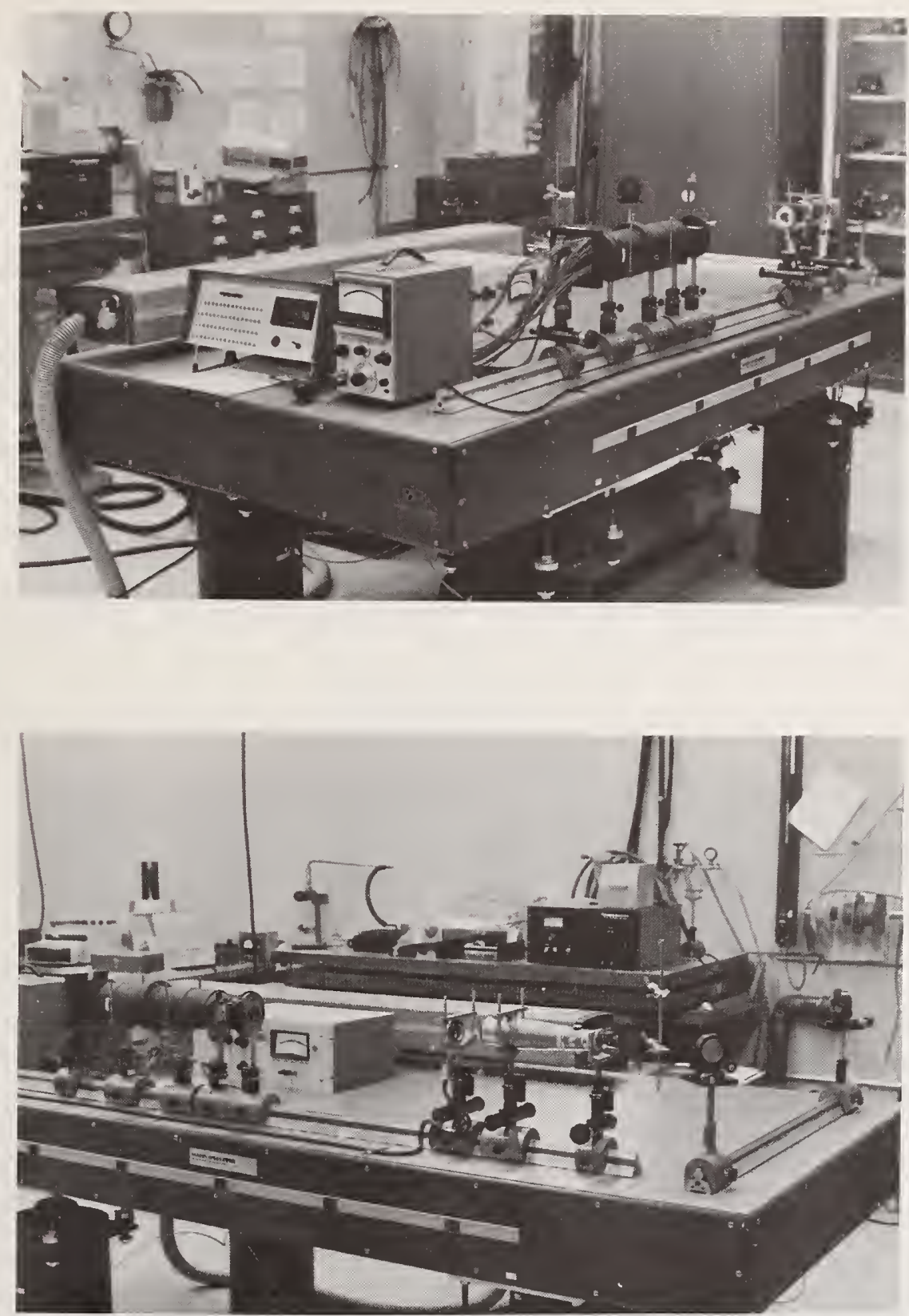

Figure 2. Two views of the optical transform technique setup in the laboratory. 

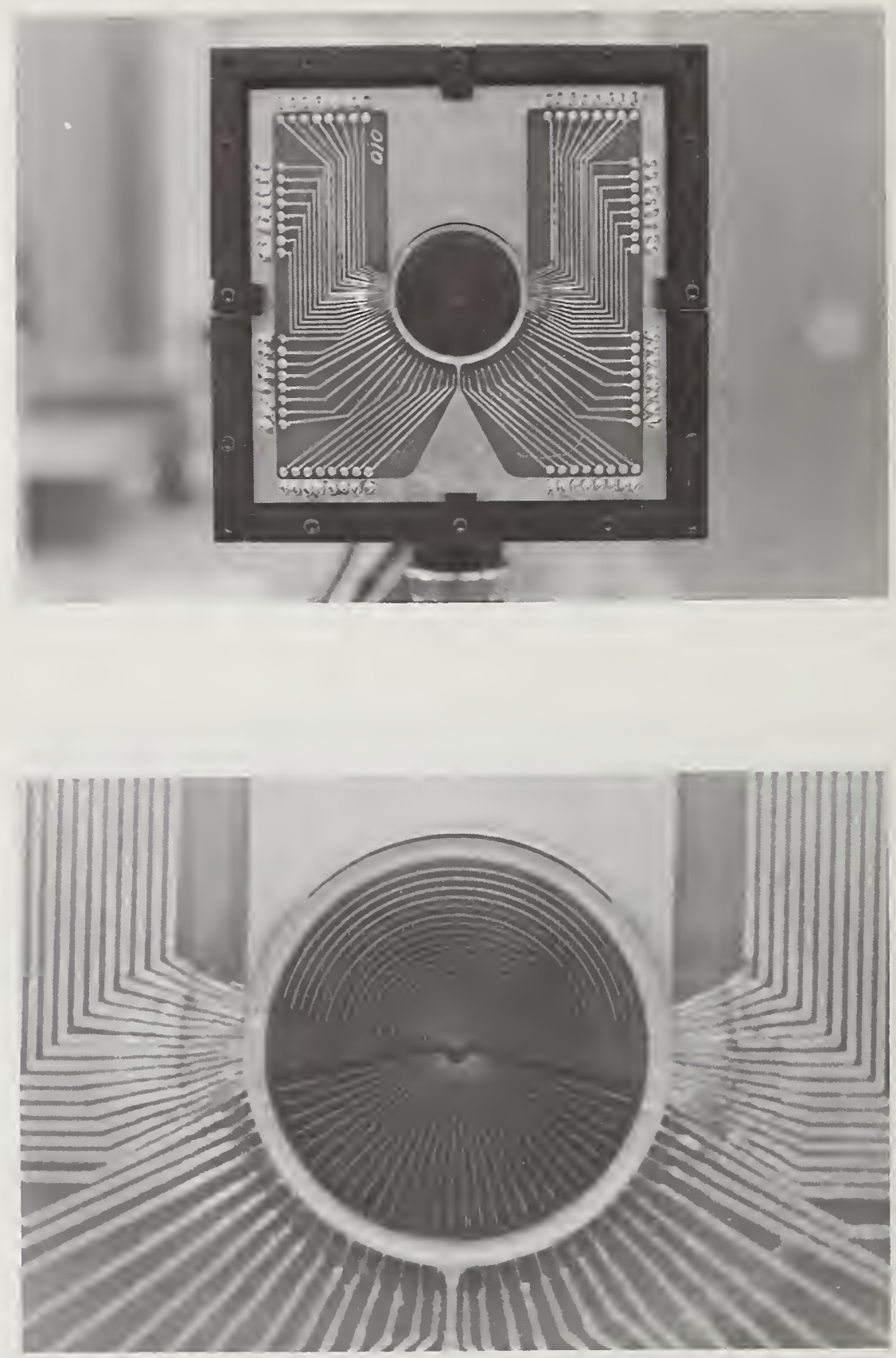

Figure 3. A commercially available photodiode array is shown in the top photograph; the circular symmetry of the array is shown in the enlargement (bottom). 

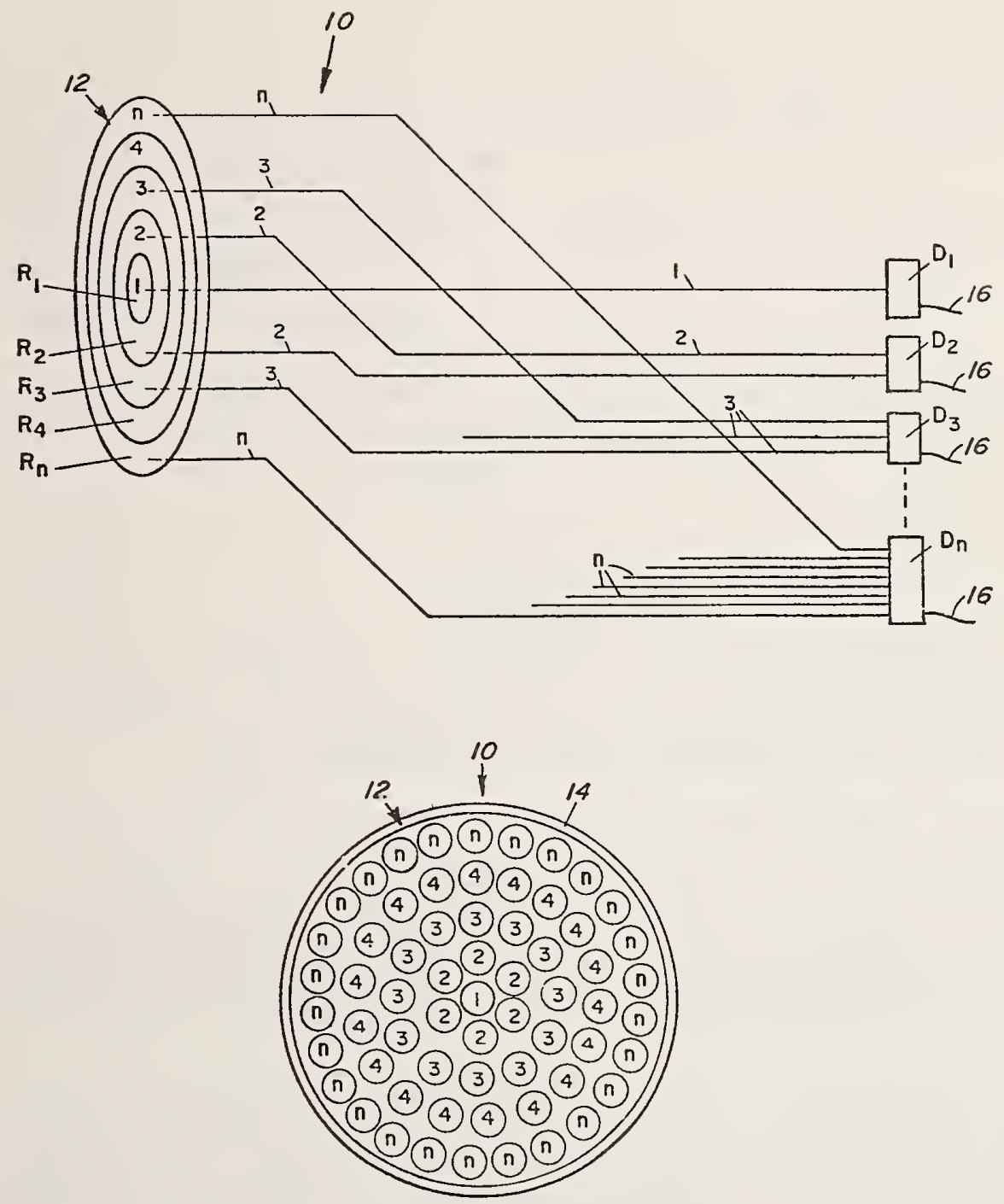

Figure 4. Diagram of a fiber-optic array circularly symmetric detector used with either photodiodes or photomultiplier tubes.

the noise in the measured data yields an estimate of the number of independent pieces of information in the inversion process [6].

After an inversion matrix has been derived, it may be applied to measured data essentially in real-time with the electronic arrangement indicated in figure 5. The output of each detector is preamplified, sampled, and held while a multiplexer feeds each signal in sequence to an analog-to-digital converter. The digitized data are then read into a computer where they are multiplied by the inversion matrix stored in the computer. 


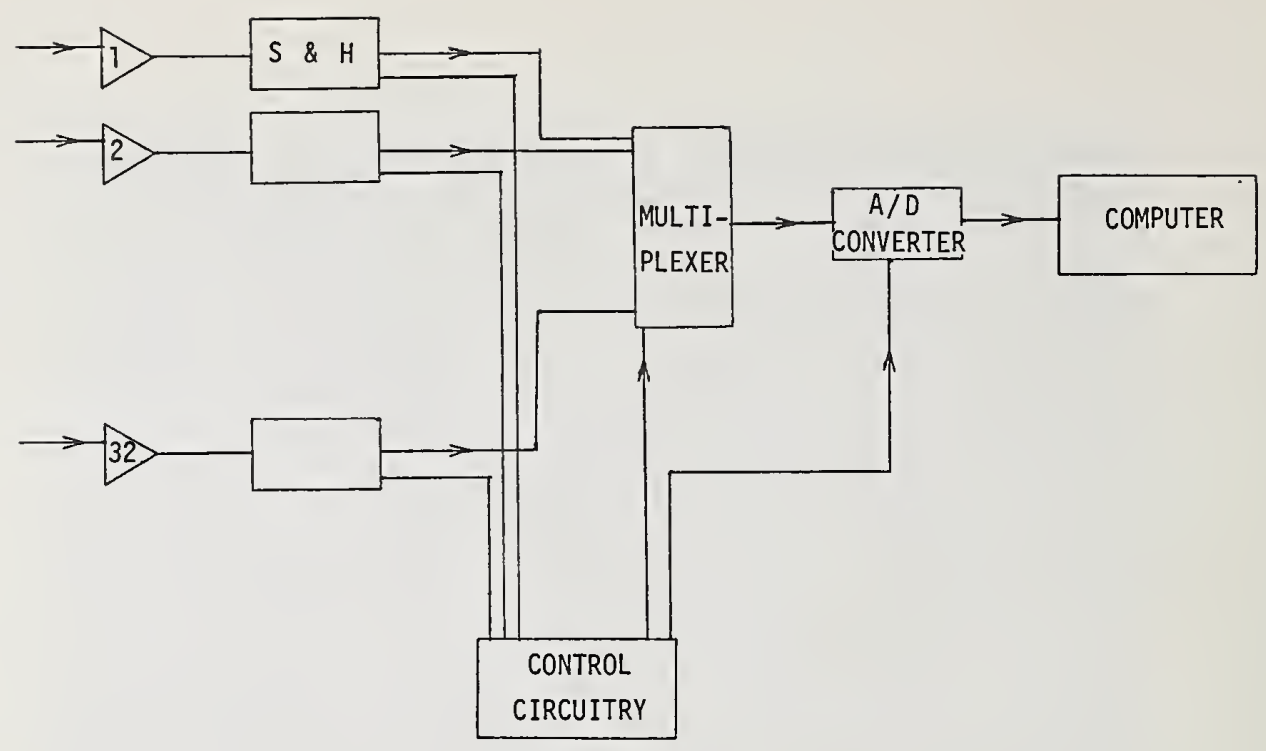

Figure 5. Schematic of electronic arrangement used for applying the derived inversion matrix to measured data.

\section{RESEARCH EFFORT}

Our present work, sponsored by EPA, is directed toward demonstrating that the technique described here can be successfully applied to the measurement of large particles in the atmosphere.

The technique is being tested with both theoretical calculations and experimental data. On the theoretical side, we are trying to determine the best size-resolution to be expected over a given range of particle sizes. Experimentally, we have measured the diffraction patterns produced by pinhole appertures in order to compare the theoretical values with experimental measurements under ideal conditions.

In the next phase of the program, an inversion matrix will be derived from measured data. In order to accomplish this, a set of monodisperse samples of known properties is required. The first set of samples will consist of photographic transparencies with opaque circular spots representing spherical particles. Samples with "particle" sizes covering the range from 17 to 104 micrometers diameter are being produced. This size range is compatible with the Recognition Systems photodiode array dimensions, the focal length of the highly corrected Fourier transform lens, and the wavelength of the laser radiation. After the technique has been successfully demonstrated in this size range, a shorter focal length lens will be substituted for the highly corrected lens to change the particle size interval to 5 to 25 micrometers diameter. At that point the technique will be tested with samples of particles in liquids and in air. 
[1] McSweeney, A., Turbidity Instrumentation: A Fiber-Optic System for Measuring Sediment Concentration by Optical Fourier Transformation, Final Report, OWRR Project No. A-027-GA, July 1971.

[2] Goodman, J. W., Introduction to Fourier Optics, McGraw-Hi11, New York (1968). [3] Anderson, W. L. and Beissner, R. E., Counting and Classifying Small Objects by Far-Field Light Scattering, Applied Optics 10, 1503, July 1971.

[4] McSweeney, A. and Rivers, W., Optical-Fiber Array for Measuring Radial Distributions of Light Intensity for Particle Size Analysis, Applied Optics 11, 2101, Sept. 1972.

[5] Cornillault, J., Particle Size Analyzer, Applied Optics 11, 265, Feb. 1972.

[6] Twomey, S. and Howe 11, H. B., Some Aspects of the Optical Estimation of Microstructure in Fog and Cloud, Applied Optics 6 , 2125, Dec. 1967.

*This project has been financed in part with federal funds from the Environmental Protection Agency under Grant Number R802214-01. The contents do not necessarily reflect the views and policies of the Environmental Protection Agency, nor does mention of trade names or commercial products constitute endorsement or recommendation for use.

\section{DISCUSSION}

MILTON KERKER: This presupposes that the intensities are going to be monotonic in size, which is okay except where you have a one-to-one relationship when you convert your intensity to size.

MCSWEENEY: No. This is analogous to Fourier transform spectroscopy where--I'm not sure how to explain the answer to your question. Perhaps I don't understand the question.

KERKER: You're measuring the distribution of light intensity.

MCSWEENEY: I think the answer is that we are not limiting ourselves to the central part of the diffraction pattern. The system should work when we are measuring particles of the sizes that give more than one lobe in the diffraction pattern. So what's happening is that the shape of the diffraction pattern is changing across the detector area as a function of size. So it's not really a monotonic function of size, and what it's analogous to is resolving the total diffraction pattern into the number of patterns similar to this as is done in Fourier transform spectroscopy. The question of resolution comes in 
even though we are making 32 light intensity measurements with this commercially available detector array. Of course you don' $t$ end up with 32 size intervals, and one of the things we are trying right now to use is the eigenvector analysis to determine the number of subintervals that we can resolve, and the upper and lower limits of each subinterval in a given size range. Now we've built a fiber optic array that consists of 168 annular rings concentric with the center fiber. Now this would give us about 5 times the spatial resolution in the light intensity measurements that we could make and, analogous to Fourier transform spectroscopy, by sampling at a much finer interval in.the diffraction pattern we could measure size distributions that consisted of larger particles. As we improve the rate of sampling in this plane we increase the size range we can measure. By increasing the diameter of the bundles, we could go down lower in size.

ILAN CHABAY: How is this detector array related to the width distribution that you are able to deconvolute from your data? What limits the width of the polydispersion in the sample?

MCSWEENEY: It's both the widths of the annular rings in the detector and the diameter of the detector array, as well as, of course, the focal length of the transform lens and the wavelength of light used.

CHABAY: Within the particle size range that you can measure, let's say you are looking at a polydisperse system, can you resolve the fine changes in distribution?

MCSWEENEY: I hope so. This is, of course, one of the tests we will have to use on the systems. Right now we are getting to the point of taking the data to derive an inversion matrix from experimental data, so we are still working partially on the eigenvector analys is to determine the maximum resolution that we can achieve with the equipment that is available now.

CHABAY (added comment): This Fourier Transform (FT) technique offers a faster method of doing classical light scattering measurements due to its multiplex advantage. The information obtained is intensity as a function of spatial position rather than intensity as a function of angle (for each angle studied one at a time) as in classical scattering. Thus, FT data contain the same amount of information as do classical methods and the same problems of data inversion are involved. 


\title{
SIZING AEROSOLS IN REAL TIME \\ BY PULSING UV LASER MACHINE
}

\author{
Geoffrey A. Hotham \\ Laser Holography Inc. \\ Santa Barbara CA 93108
}

\section{ABSTRACT}

A new laser machine will be described which utilizes a pulsing UV laser to produce "instantaneous" images of aerosol droplets on a TV screen in real time. With this instrument it is possible to measure from 0.3 to 1000 micrometers.

Key words: aerosol impact studies; aerosol sizing aerosol spectrometer; aerosol sprays; laser holography; laser imaging of particles.

\section{LASER MACHINE}

What you will see will be a video tape that depicts a new laser machine that presents spray-drop pictures in real time. Figure 1 shows the pulsing laser machine on the left interfaced with automatic scanner on right. Instantaneous pictures of spray drops are presented while they are happening $[1,2]$.

The spray is simply left on and a high speed laser is continuously pulsed through the spray producing instantaneous spray-drop pictures on the TV screen.

The new system uses a continuously pulsing laser, requires no film or wet developing or any reconstruction. This laser machine produces spray-drop pictures in real time. They are recorded in an instant replay recorder. The pictures are replayed and sized by a reticule on the face of the monitor.

This machine is useful for sizing the aerosols of hairsprays, oven cleaners, deodorants, air fresheners, insecticides and all household goods. The quantity of respirable particles in aerosol sprays may be readily determined. Inhalation toxicity may be predicted. Such things as size distribution, agglomeration, coalescence, evaporation, air pollutants may be obtained and studied in real time.

The size range capability of this machine is from .3 micrometers to 10,000 micrometers. Size distribution is plotted in steps as follows: 
MONITOR

LASER

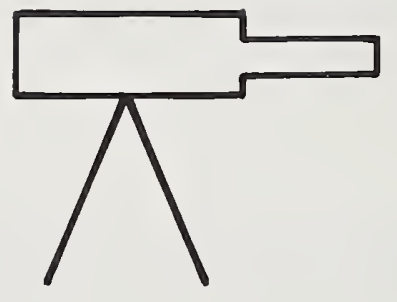

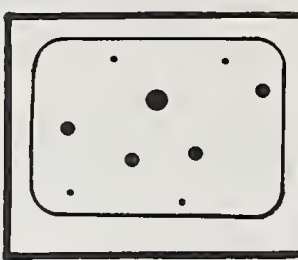

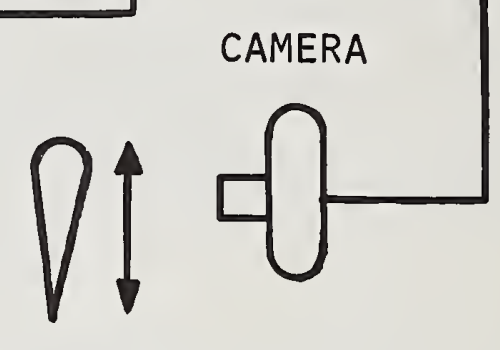

SPRAY

Figure 1. Pulsing laser machine and automatic scanner.

.3 micrometers to 1 micrometer; 1 micrometer to 5 micrometers; 5 micrometers to 10 micrometers; 10 micrometers to 20 micrometers; and so on.

The velocity of measurable particles is stationary to 1,000 feet (300 meters) per second. The types of particles measurable include all aerosols, liquid droplets, powders, particulates, tubular structures, crystals, touching particles, doughnut constructions, hollow spheres and balloons.

\section{LASER SET-UP}

This is the laser set-up (fig. 2). I will identify the various parts; the laser, the spray, the lens, the camera, and the monitor. Next, I will turn the spray on. The can may be moved in and out to study drop sizes at different positions from the nozzle (figs. 3 and 4).

We also offer an automatic scanner that interfaces with this laser machine providing size distribution while the spray is going on; i.e., in real time. Counts may be made at rates of 5,000 particles per second to as high as 10,000 particle counts per second (fig. 1).

Size distribution is shown in real time by way of nixie tubes displaying accumulated totals in each size range.

\section{VIDEO PRESENTATION}

Most of my presentation today will be made via video tape. You will see video pictures of aerosol particles in flight. Also, I will 


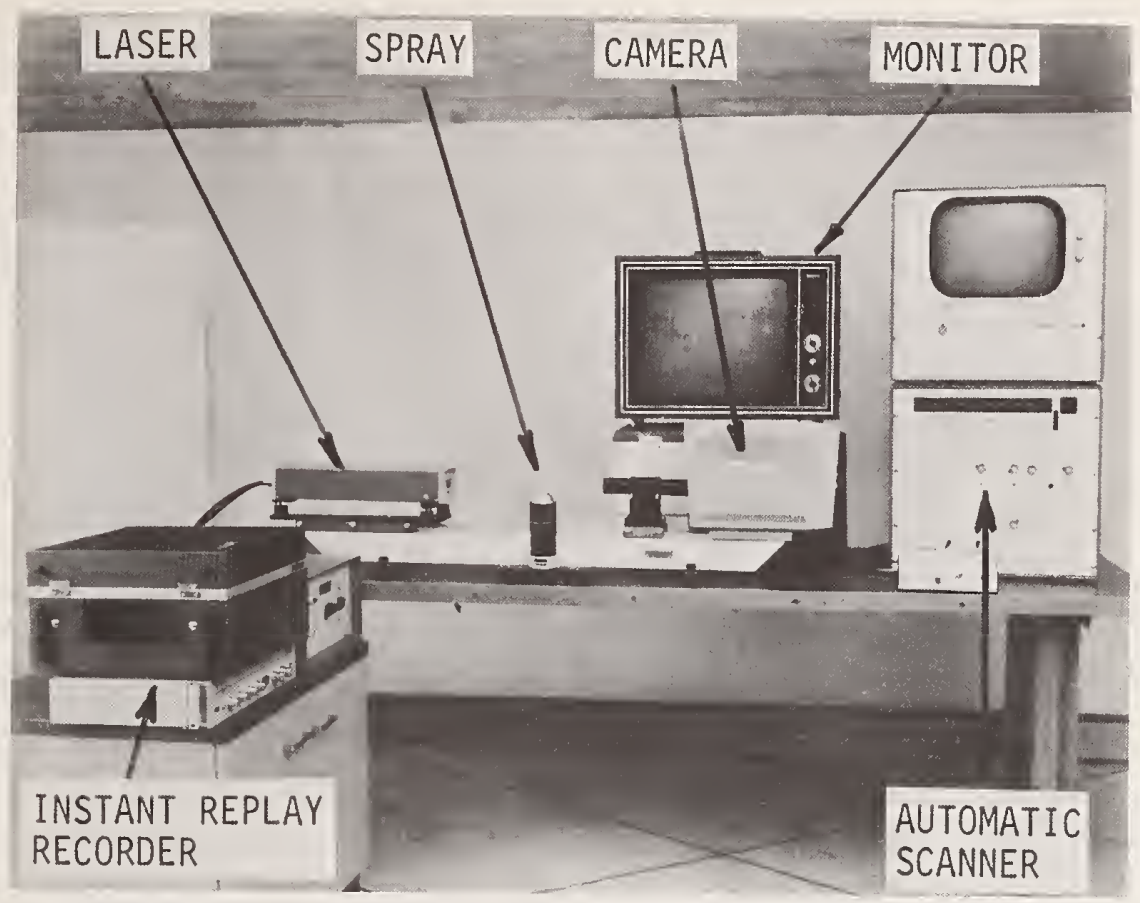

Figure 2. Schematic of laser setup.

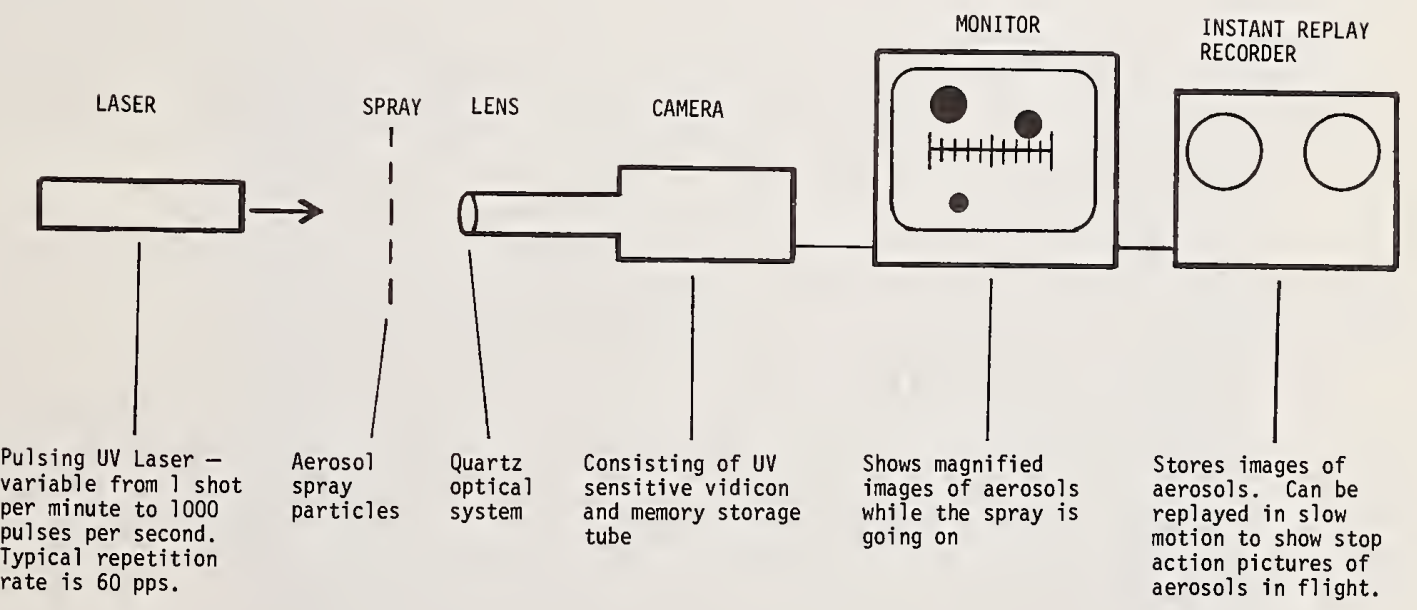

Figure 3. Pulsing laser machine showing laser, spray, camera, monitor and instant replay recorder. Instantaneous particle pictures are generated on the monitor while the spray is going on. These pictures are stored in an instant replay recorder and may be played back in slow motion. Size of the particles is determined by eye by the reticle on the face of the monitor. Size range measurable 0.3 to 10,000 micrometer. 


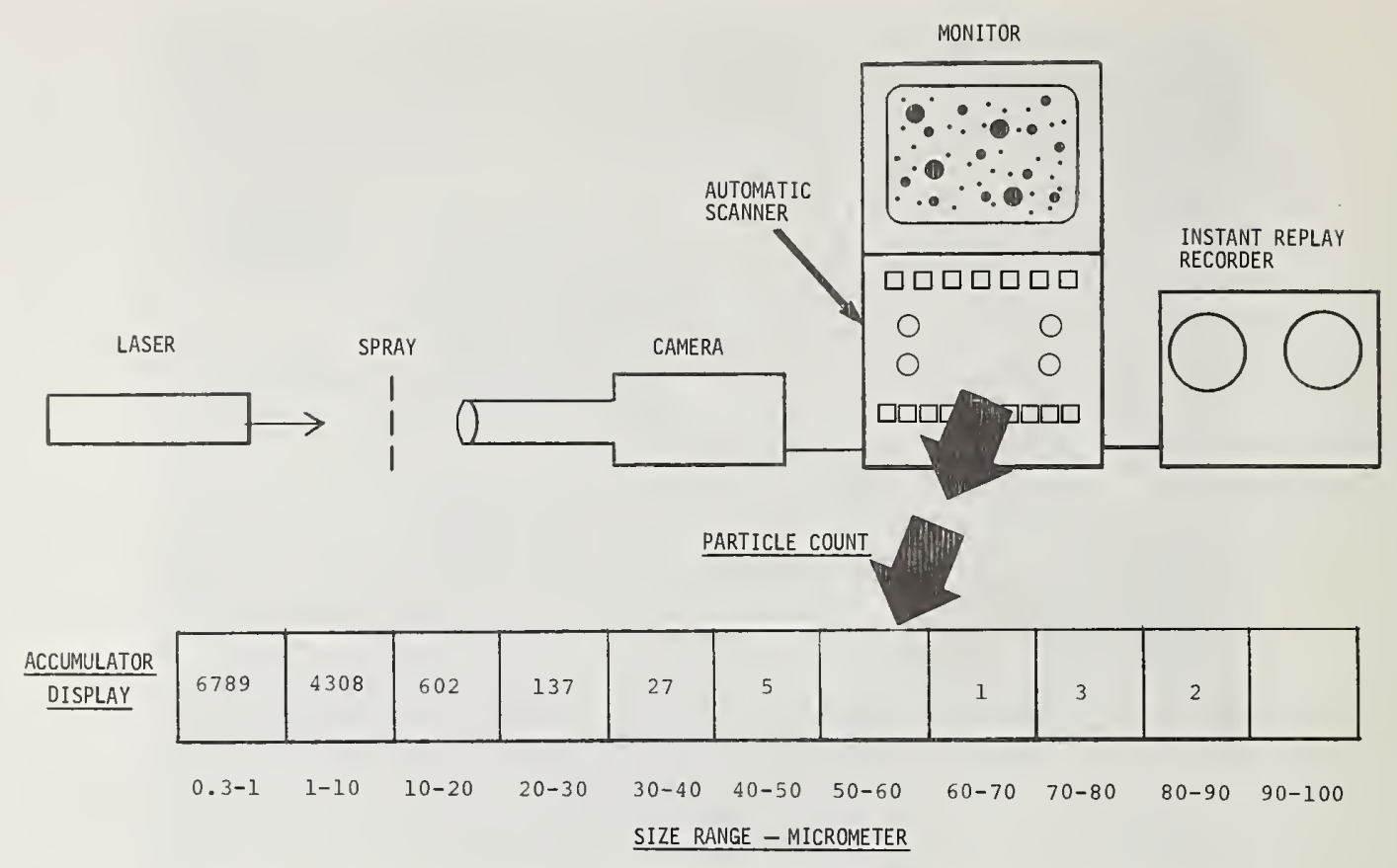

Figure 4. Pulsing laser machine and automatic scanner snowing enlarged view of accumulator display of particle counts. Size distribution in real time scans images of particles and automatically sizes them in real time while the spray is going on. Counts of particles in various size ranges are registered in the accumulator display. Capable of sizing particles and counting them at rates of 10,000 particles per second. Accumulated counts are shown in the accumulator display while the spray is going on.

show excerpts from various contracts that I have performed with this equipment.

To start with I will show some large drops recorded by this machine. These pictures will show their size, shape, and outline their breakup (fig. 5).

\section{WATER SPRAY DROPLETS}

What you see now are some water drops being sprayed from a pneumatic nozzle. We are looking at the spray pattern 4 inches $(\sim 10 \mathrm{~cm})$ downstream or 4 inches $(\sim 10 \mathrm{~cm})$ from the nozzle. Those particles are quite 1 arge-- 1 inch $(\sim 2.5 \mathrm{~cm})$ on the monitor represents 200 micrometers.

We're looking at the general spray pattern at 4 inches downstream. The laser is fired through the spray, the camera is generating those spray-drop pictures, and they are being recorded in the instant replay recorder. 


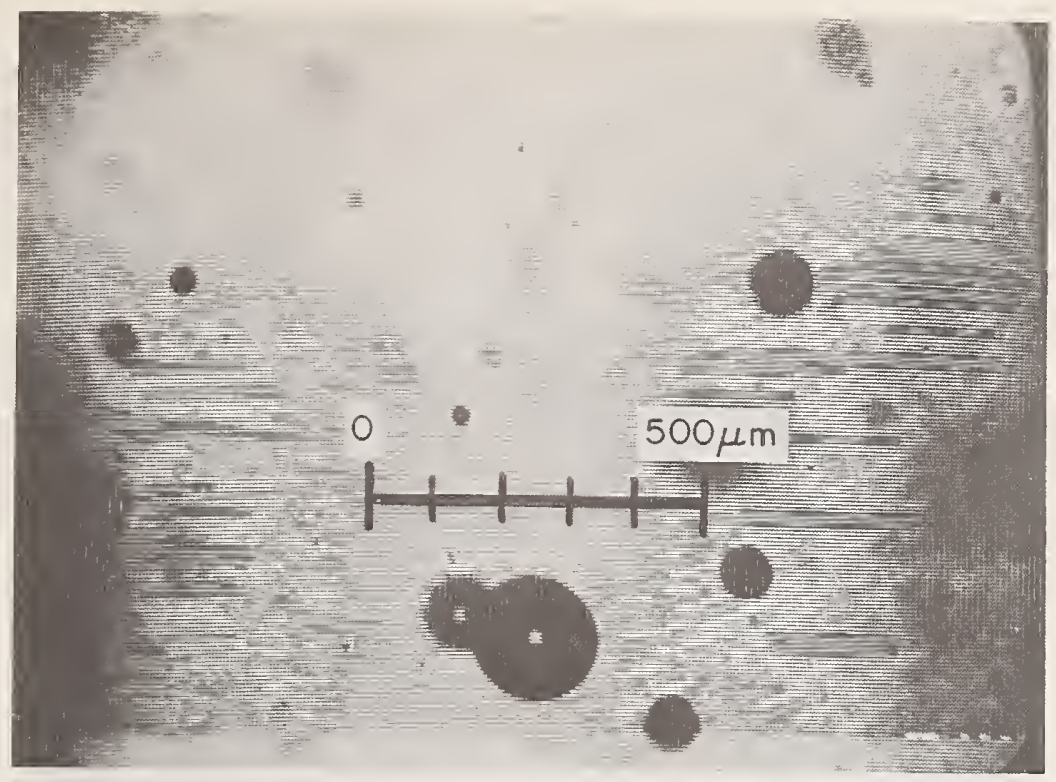

Figure 5. Water drops sprayed from a pneumatic nozzle.

These droplets are fairly round; most of them are round, some of them are odd-shapped--elliptical and dumbbell shaped (fig. 6).

We'll move the camera nearer to the nozzle and see the actual breakup of the water jet into discrete particles.

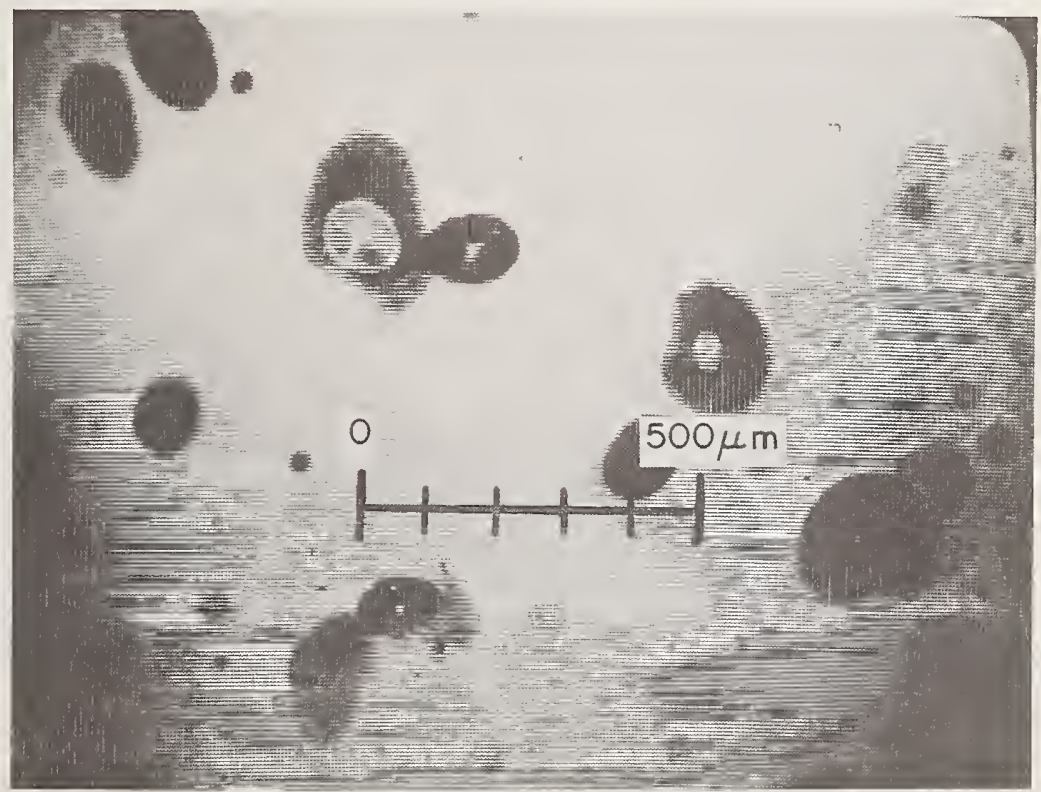

Figure 6. Water droplets are mostly round in shape-some are elliptical and dumbbell shaped. 
The camera and laser have been moved to look at that point where the liquid jet breaks up into discrete particles; it is at the rupture point of the liquid (fig. 7).

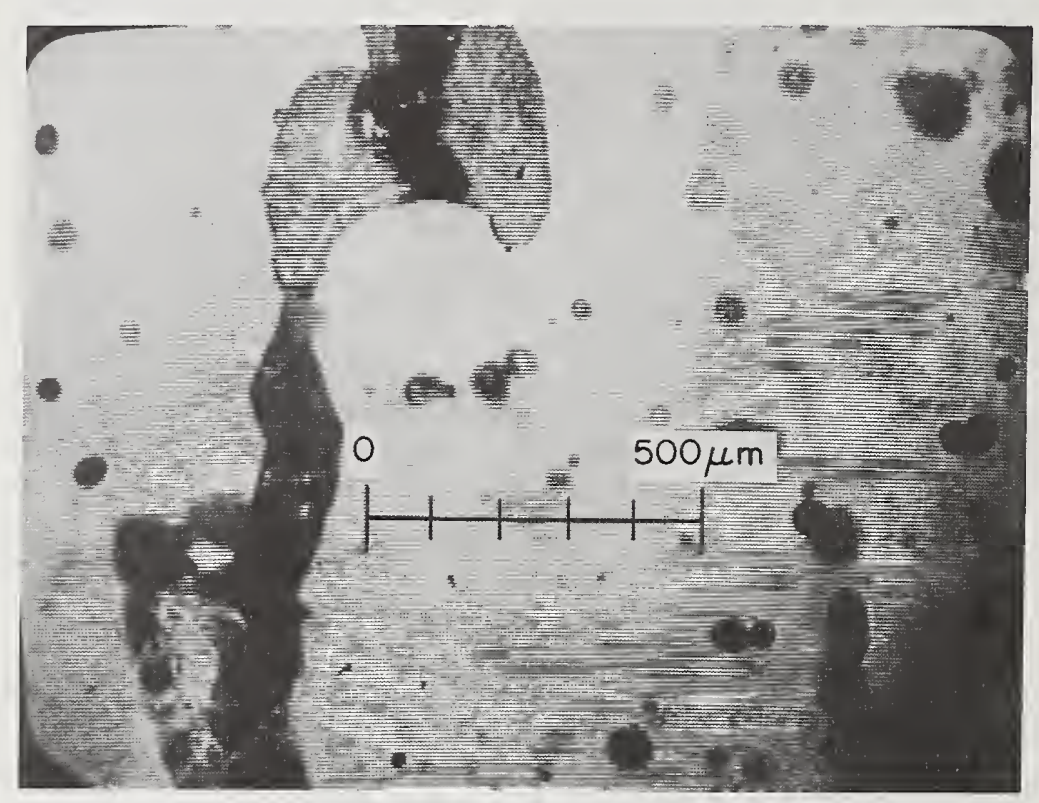

Figure 7. Rupture point of the liquid--the liquid jet breaks up into discrete particles. to breakup.

We are looking at the filaments and ligaments of liquid just prior

The laser is being fired at 10 pulses per second, and each one of those pulses is capturing a picture of the breakup of the particle.

The width of the laser pulse is 10 nanoseconds so it, for all intents and purposes, makes a stop-action picture of the particles at that instant (fig. 8).

You can see that the jet is broken up into discrete particles at this point. This is about 1 inch $(\sim 2.5 \mathrm{~cm})$ downstream. Those are the discrete particles now in the stream.

We are moved downstream and we are looking for the actual rupture point and this is it, and this is where the jet is breaking up into discrete particles (fig. 9). (I'll play that in slow motion.) That is frame by frame and that is the liquid right at its final point before it breaks up into discrete particles. 


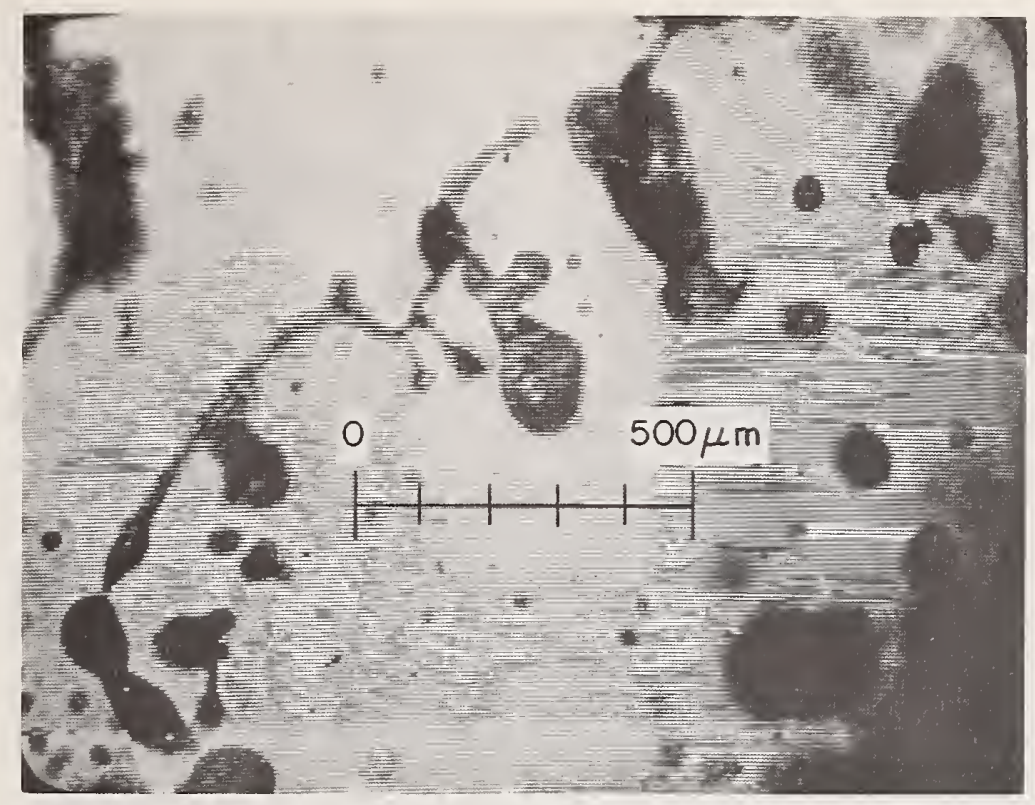

Figure 8. Stop action picture of the particles at the instant the laser pulse width is 10 nanoseconds.

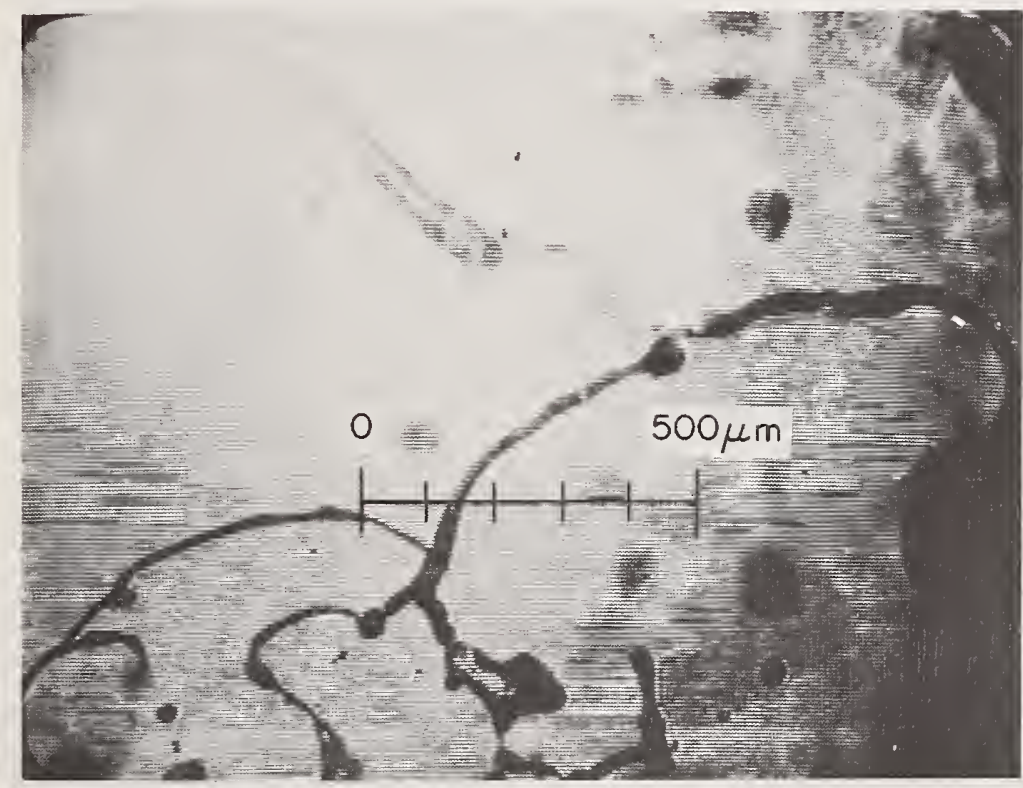

Figure 9. Actual rupture point of the liquid. 
Those are the filaments of the liquid just prior to breakup (fig. 10). The jet is moving from left to right and a few milliseconds later each one of those filaments will be broken into discrete particles.

The jet is going from left to right and the laser is fired normal through the axis of the spray and we're looking at the end of the liquid jet (fig. 11). This is the actual mechanics of the breakup of a liquid jet into discrete particles.

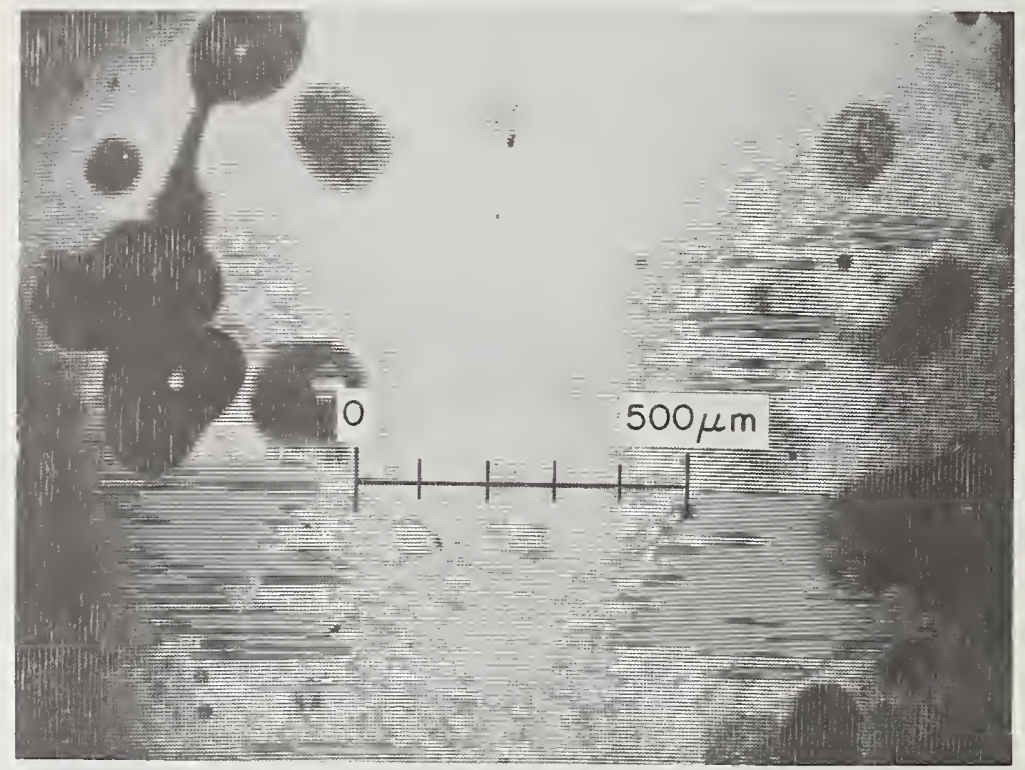

'Figure 10. Filaments of the liquid just prior to breakup.

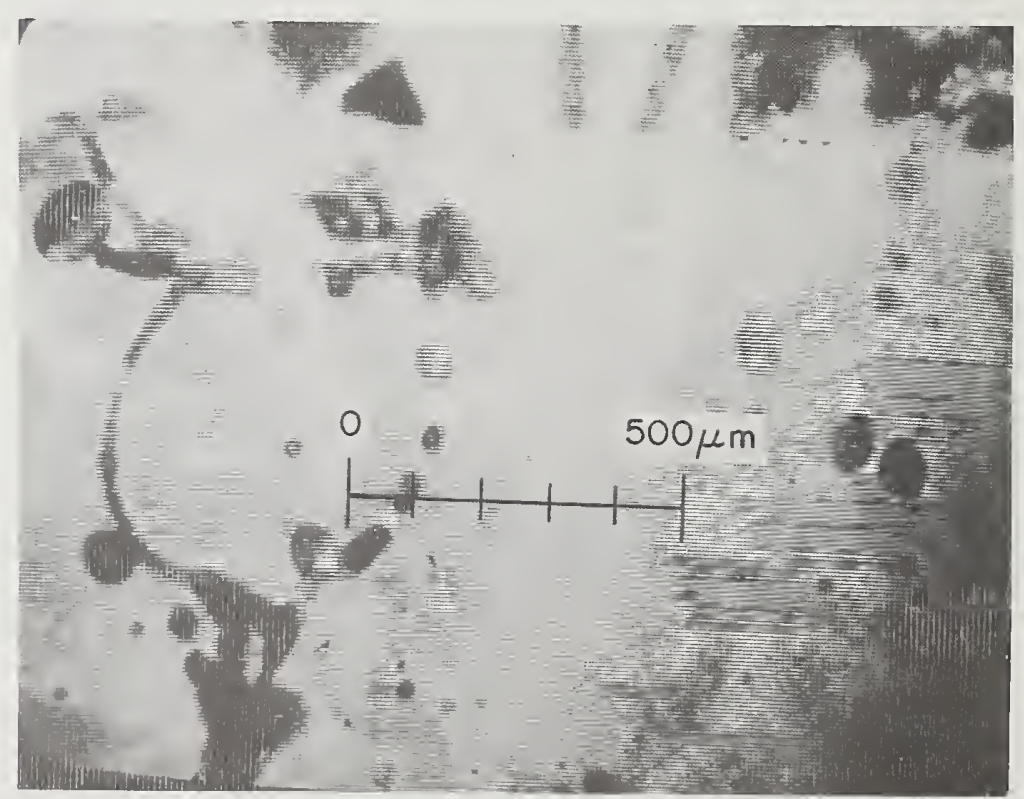

Figure 11. Looking at the end of the liquid jet. 
Calibration is performed with a microscope reticule placed in the plane of the spray prior to spraying. It is recorded in the instant replay recorder. It is then removed from the optical bench. Its image may be superimposed over the spray particles during spraying.

\section{TIME GENERATOR}

A time generator is added to the system to provide a record of the date of the test for legal reasons: month, day and year (fig. 12).

It also provides time: hours, minutes and seconds (and tenths of seconds if necessary) for studying such things as evaporation, decay, agglomeration, etc.

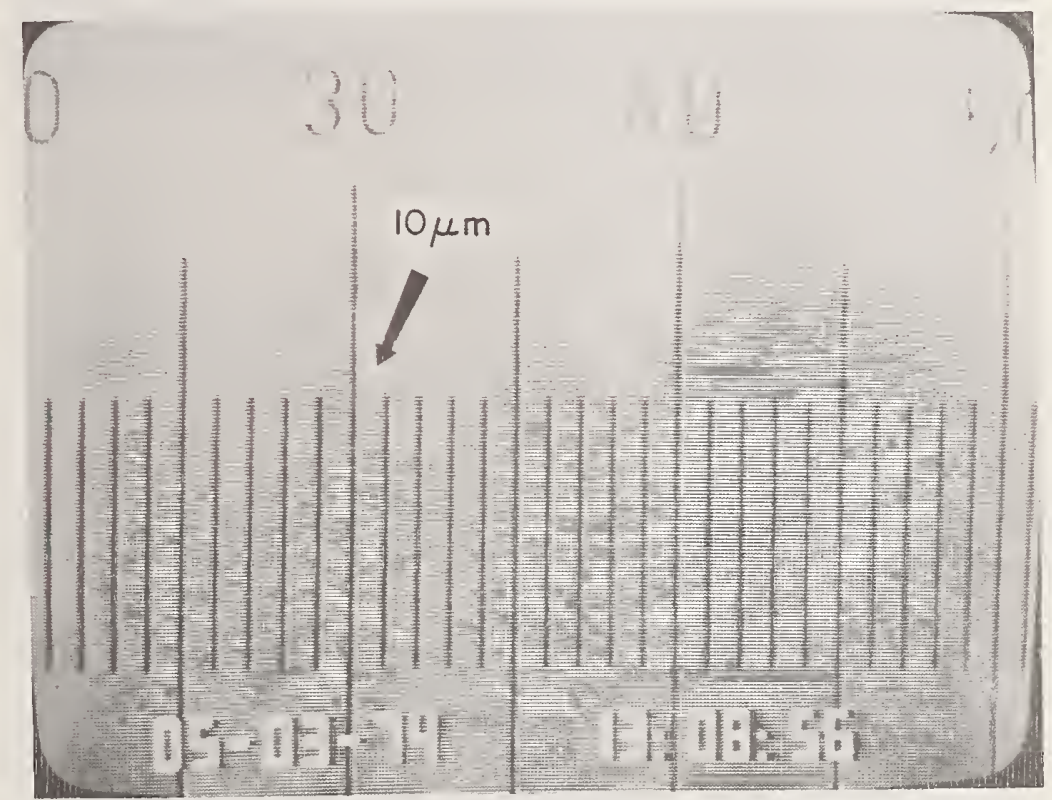

Figure 12. Time generator added to system.

ANTIPERSPIRANT. IMPACTION.

This next frame (fig. 13) will show an antiperspirant sprayed onto a 5-micrometer target strand. That strand is 5 micrometers in diameter and you' 17 see particles impacting onto that strand. That is the first impaction. The spray was directed at that strand from 10 inches ( 25 $\mathrm{cm})$ away. There' 17 be another spraying in a few seconds. This is the replay recorder replaying those scenes that we just saw. In a few seconds you'll see the first hitting of the particles and you'll see particles flying around those impaction strands. In a few seconds you' 71 see the first hitting of the antiperspirant. Target strand is 5 micrometers. 


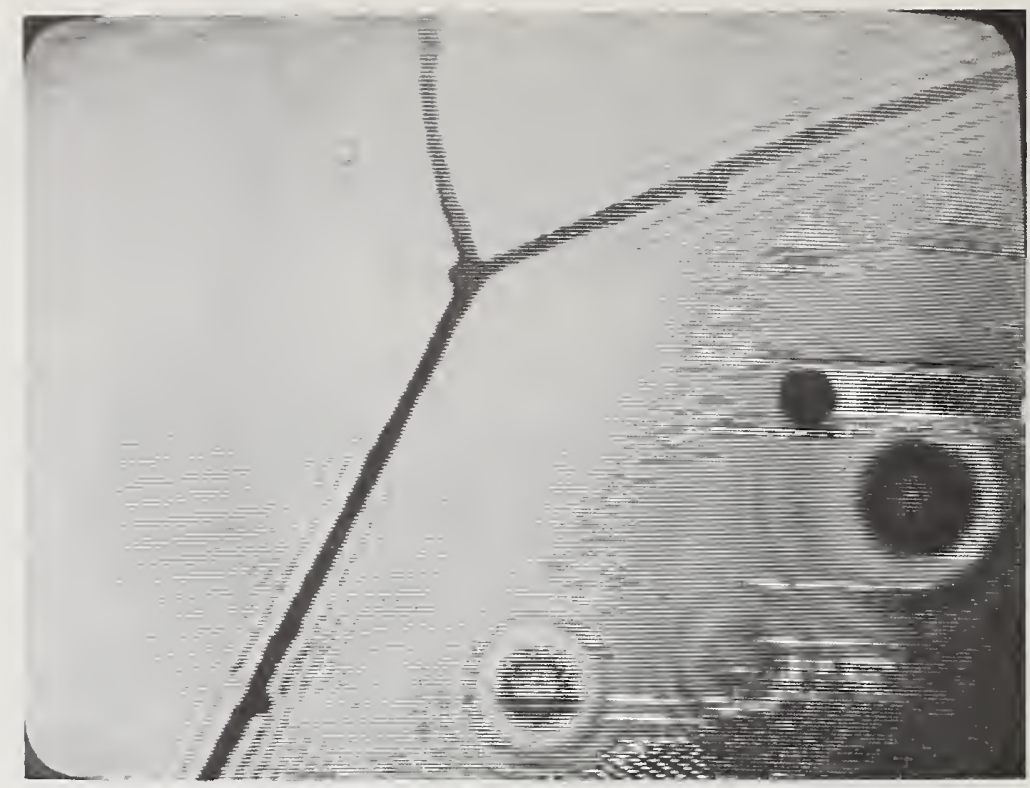

Figure 13. Antiperspirant sprayed onto a 5 micrometer target strand.

Those are the particles and that is the first impaction. On playback we see the first hitting of the particles. Rolling the recorder reel back we are looking at the first arrival of the antiperspirant.

The next scenes are all taken from the instant replay recorder. The laser is being fired through that scene and as the particles impact you will see them coming on the screen (fig. 14). That's an antiperspirant.

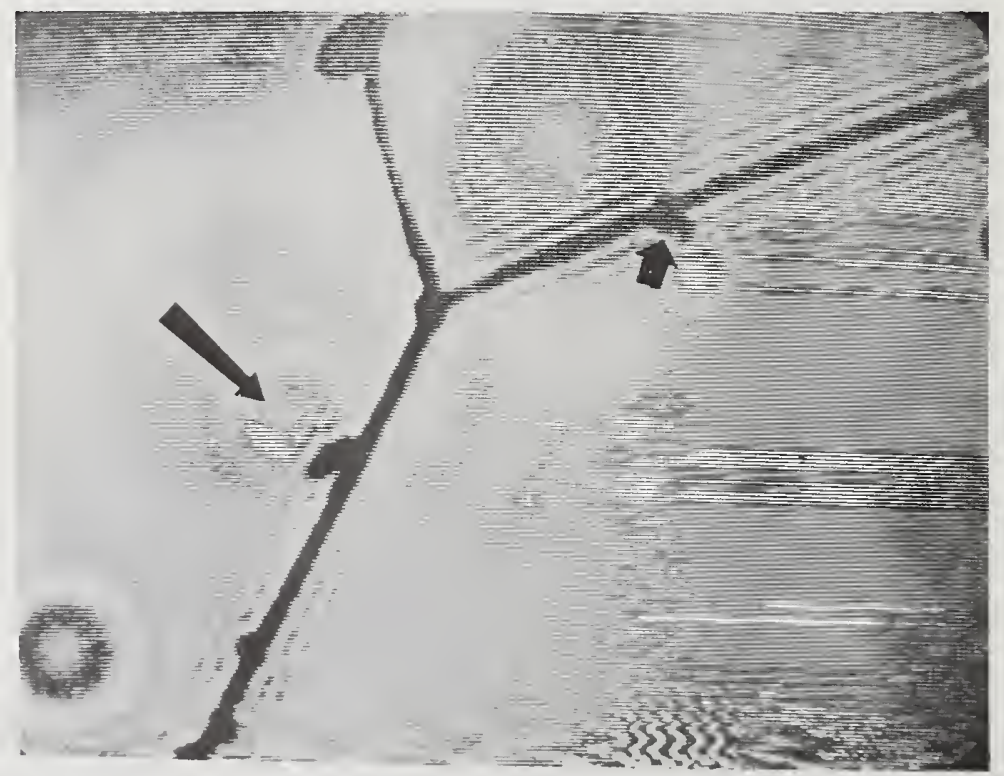

Figure 14. Frame showing particles coming into screen as they impact with laser pulse. 


\section{ANTIPERSPIRANT. SIZE.}

The strand is 5 micrometers and if you remember from the calibration, the smallest division in the calibration reticule was 10 micrometers, so that gives you an idea of the size of the flying particles. The magnification is $2400 \mathrm{x}$.

That is a second spraying and you can see the buildup and agglomeration onto other particles (fig. 15). That shows the buildup and impaction of an antiperspirant.

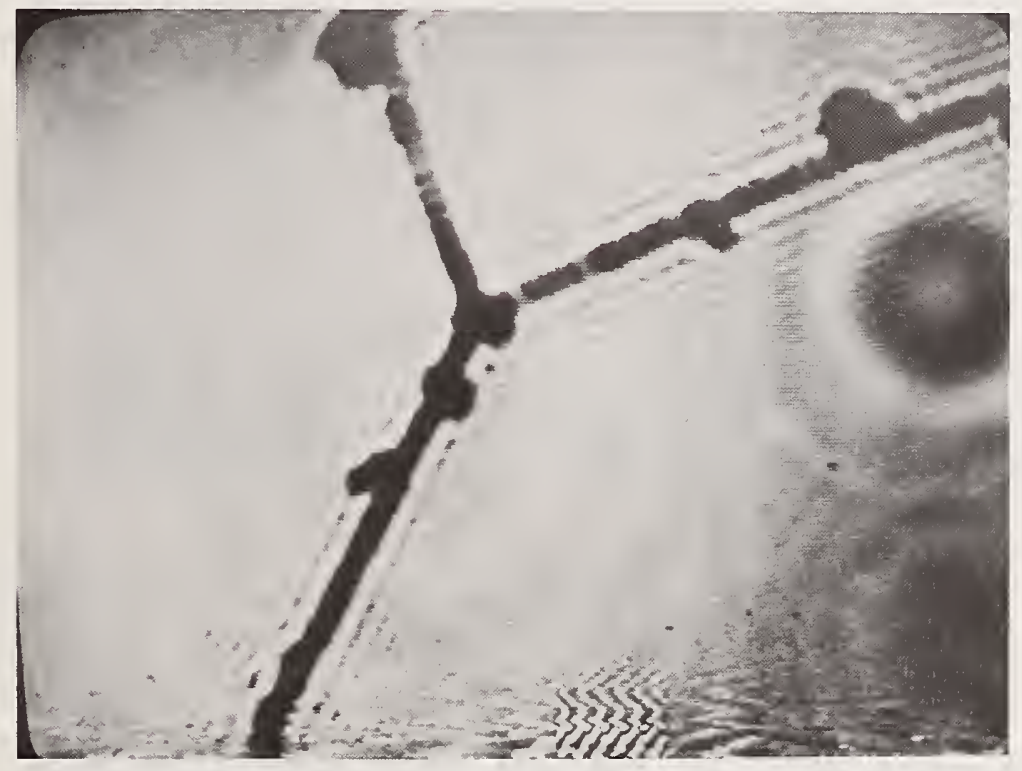

Figure 15. Buildup and agglomeration onto other particles (second spraying).

\section{DEODORANT. AT THE NOZZLE}

The next spraying is a deodorant. The photo shows the spray at the nozzle (fig. 16). 


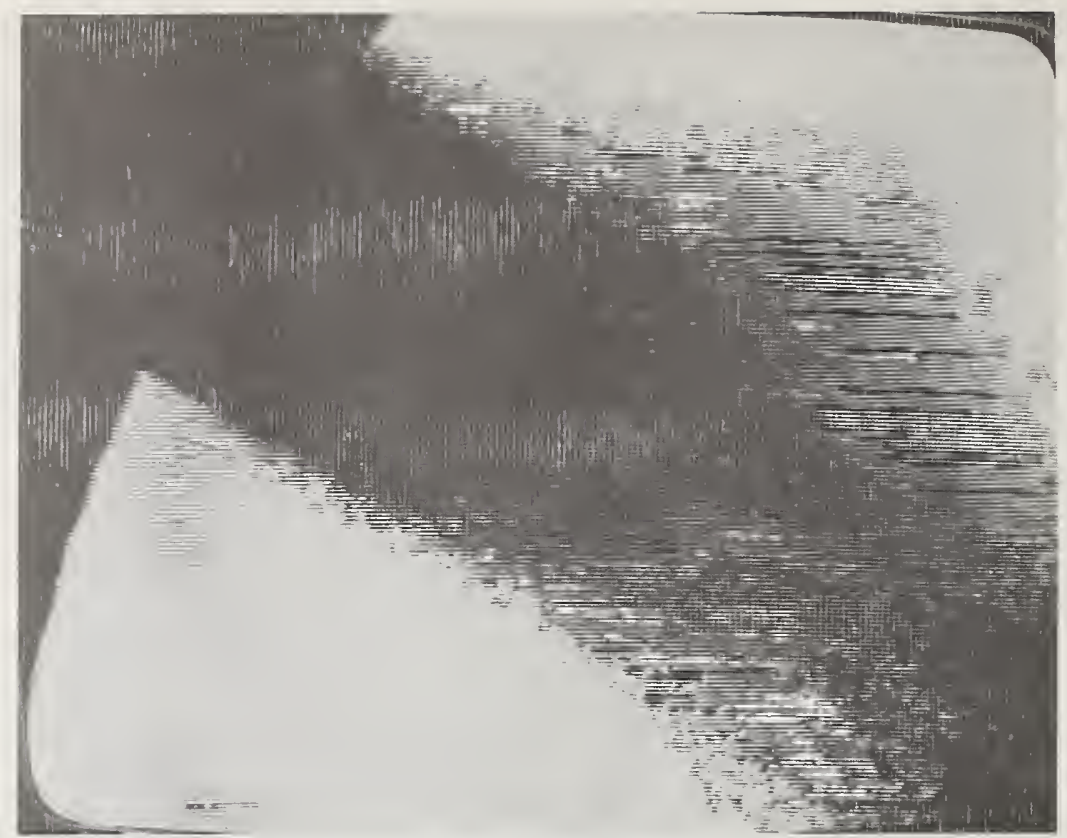

Figure 16. Frame showing spray at the nozzle.

DEODORANT. FALL OUT

These are some large drops that fall out along the bottom of the spray (fig. 17). They, of course, reduce the efficacy of the formulation.

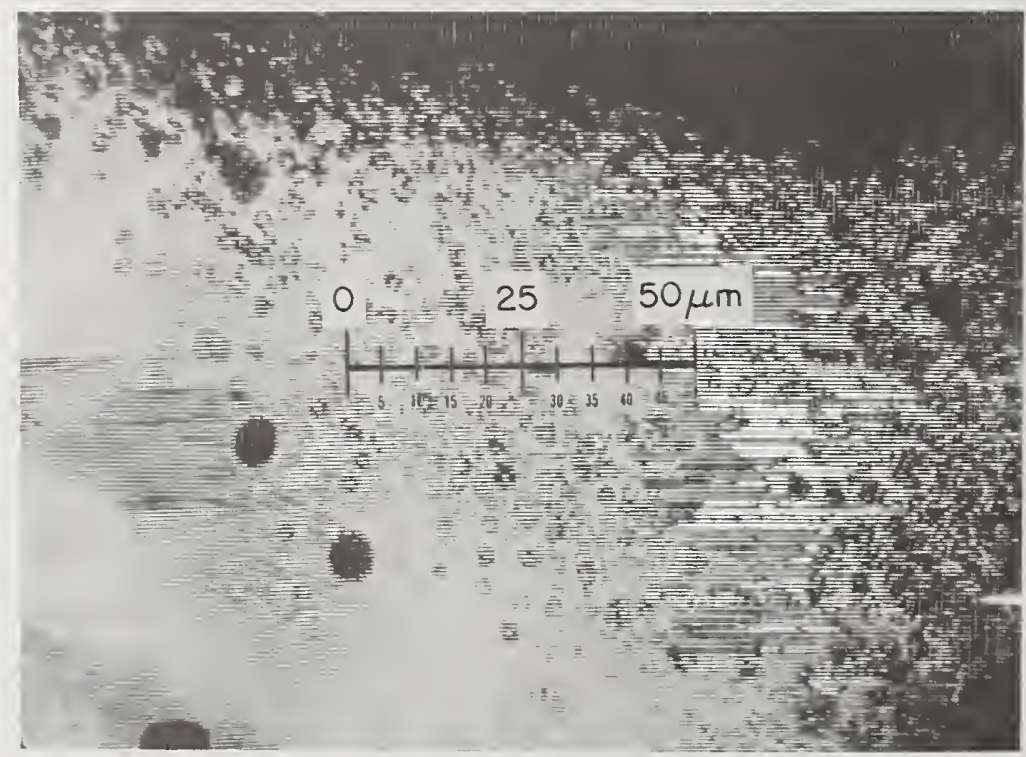

Figure 17. Large drops fall out along the bottom of the spray. 
This scene is taken 8 inches $(20 \mathrm{~cm})$ downstream from the nozzle (fig. 18). It shows the particles in free flight. It can be seen from the reticule that all the particles are quite small--all below 10 micrometers in size.

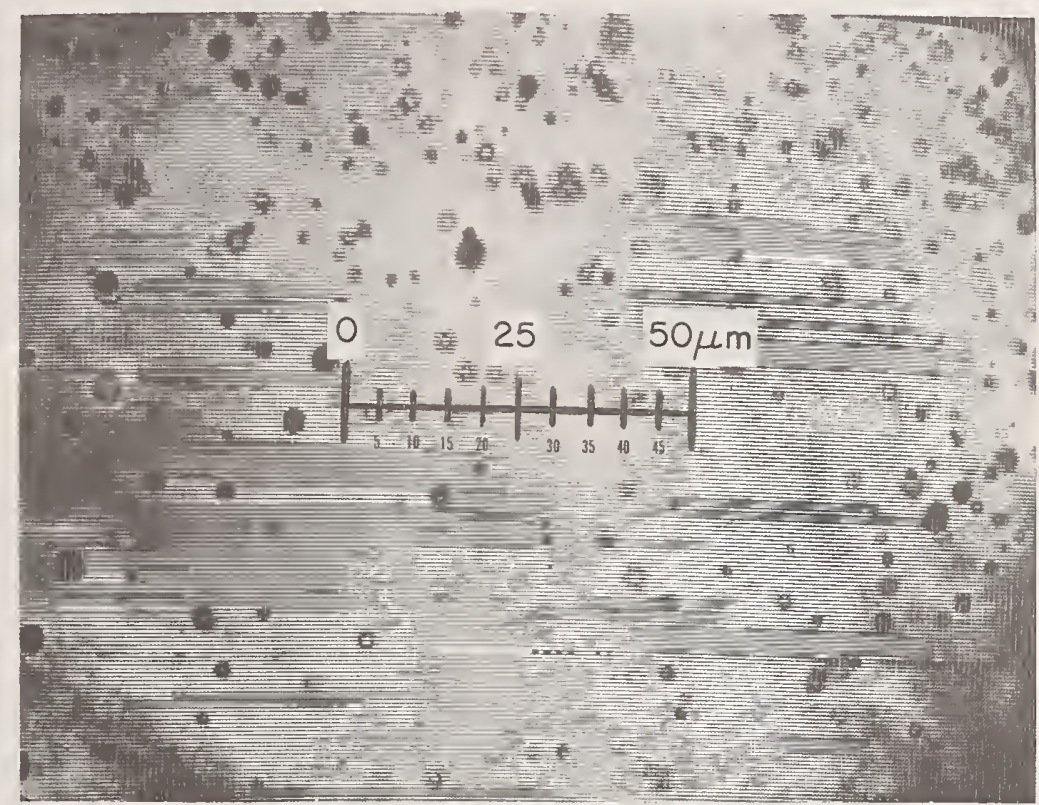

Figure 18. Frame taken $20 \mathrm{~cm}$ downstream from nozzle shows particles in free flight.

DEODORANT. REAL TIME SIZE DISTRIBUTION.

This information may be sent directly to the Automatic Scanner, and size distribution is obtained immediately. Counts of particle size are made at rates of 5,000 counts per second. The accumulated totals are shown in real time on the Accumulator Display. Hence, size distribution is obtained in real time while the spray is going on.

Accumulated total counts of particles are displayed in size ranges as follows: $0.3-1$ micrometer, $1-5,5-10,10-20,20-30,30-40$, up to 100 micrometers or higher. See the drawing outlining the Automatic Scanner and accumulated display of particle counts for real time size distribution (fig. 3 ) $[3,4]$.

\section{AUTOMATIC SCANNER}

This scanner automatically sizes only the in-focus particles. Outof-focus particles appear grey and have diffraction patterns around them. The scanning circuitry senses diffraction rings and does not count them. Similarly the sensitivity of the scanner is set so that it misses the grey out-of-focus particles. It only sizes and counts the solid black dots or images of particles in focus. 
Since the scanner is looking at typically 60 frames per second where there are usually about 100 particles per frame in focus, the statistical sampling is indeed reliable and representative.

The question is often asked, "What is the depth of focus of this system?" Careful measurements have been made on this. The depth of focus for a 100 micrometer particle has been determined to be $\pm 2 \mathrm{~mm}$.

\section{IN SITU}

This laser machine records aerosol spray patterns in situ--in place while they are happening. It records a 11 the particles in the scene-all of the small particles in the 0.3 to 10 micrometer range and all of the large particles, 10-20-30 micrometer size range, up to as high as 10,000 micrometers.

Most light scattering particle sizing machines use isokinetic sampling. This is, they suck in or draw in by vacuum flow only the small fine particles that are left suspended in the air. These machines measure only the airborne particles drawn into them by vacuum. They usually do not "catch" droplets above 30 or 40 micrometers. Certainly the 80 and 90 micrometer sized particles of a hair spray which go as high as 130 or 180 micrometer jam up the small entrance orifice of these isokinetic-sampling light-scattering instruments that sample particles one at a time. There is also the problem in these airborne sampling machines of impaction at the orifice as mentioned above, together with the probability of coalescence and agglomeration of particles such as an oven cleaner as they are drawn together down the entrance orifice. These orifices have to be small to try to sample particles one at a time and they are usually on the order of a few millimeter in inside diameter.

This laser machine photographs the flying particles in flight--in situ--and records all the particles in the spray, large and small.

Thus, it does not rely on sampling particles one at a time--it records all particles, not just the fine airborne particles; it does not deposit, impinge, impact or contact any of the particles during a measurement.

\section{EVAPORATION}

The next sequence shows a large drop of pure alcohol evaporating (fig. 19). It acts in a similar way to a hairspray; except that in a hairspray after the alcohol has evaporated, there is a residue left on the hair. This residue acts as a binder and supports the human hair. 


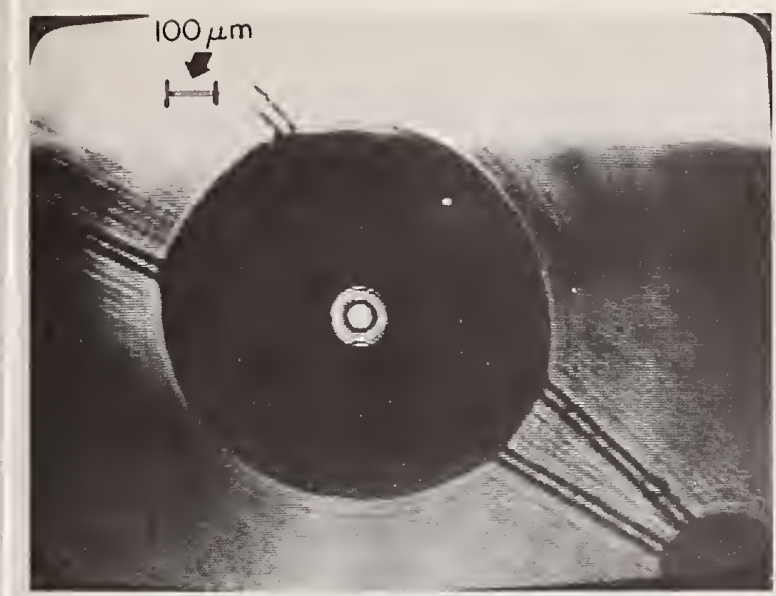

a. large drop of pure alcohol

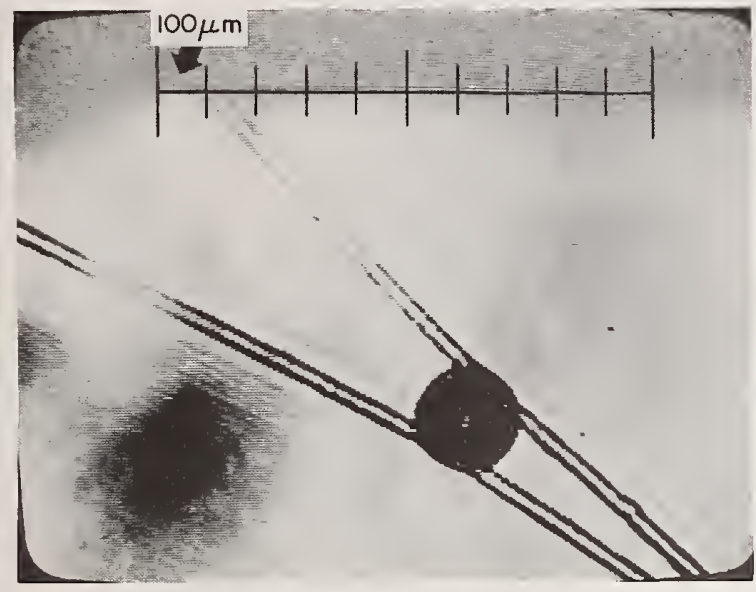

c. same drop, three seconds later

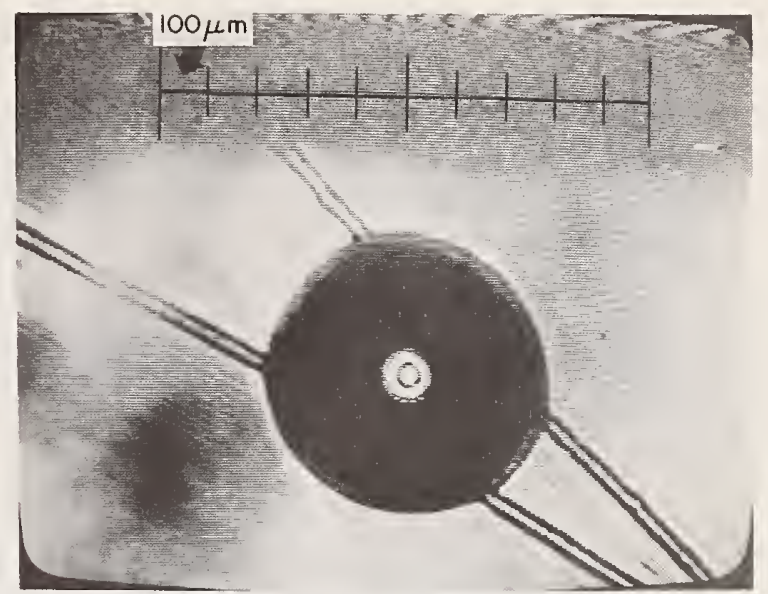

b. four seconds later

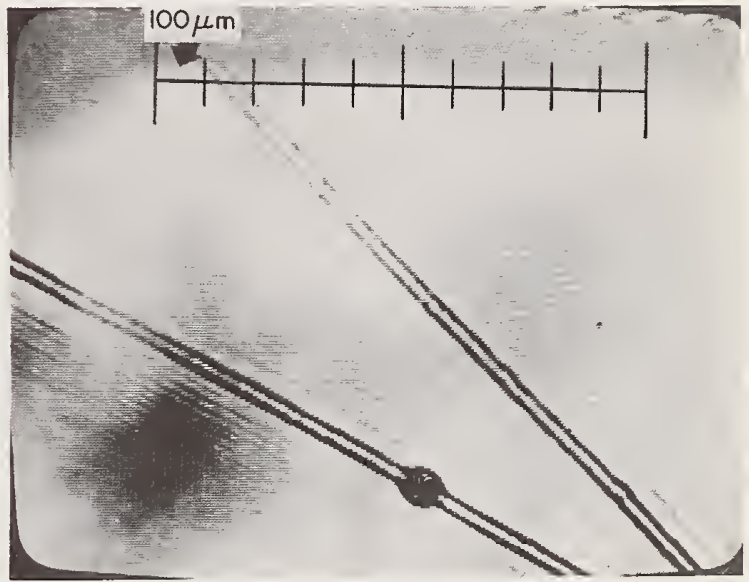

d. two seconds later

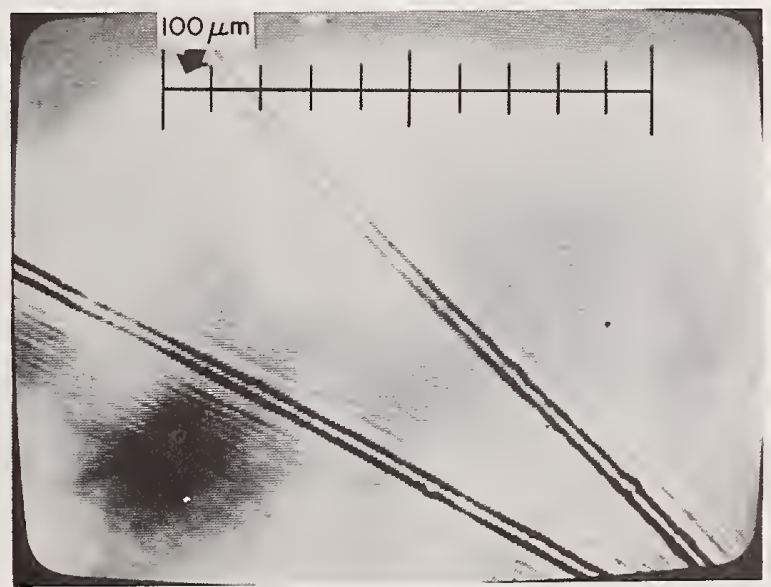

e. one second later, complete evaporation

Figure 19. A large drop of pure alcohol evaporating. 
We're going to another lens system and I want to show the calibration--1 division equals 100 micrometers. We'11 be spraying with a household oven cleaner and you' 11 see a particle impacting at $30^{\prime}$ clock (fig. 20a).

There it is there--it's expanding slowly, absorbing moisture from the atmosphere and it'11 collapse in a few seconds due to this water absorption and the residual effect of the expanding freon inside the sphere (fig. 20b).

That's the collapsed particle. It's a liquid droplet. It started as a hollow balloon (fig. 20c).

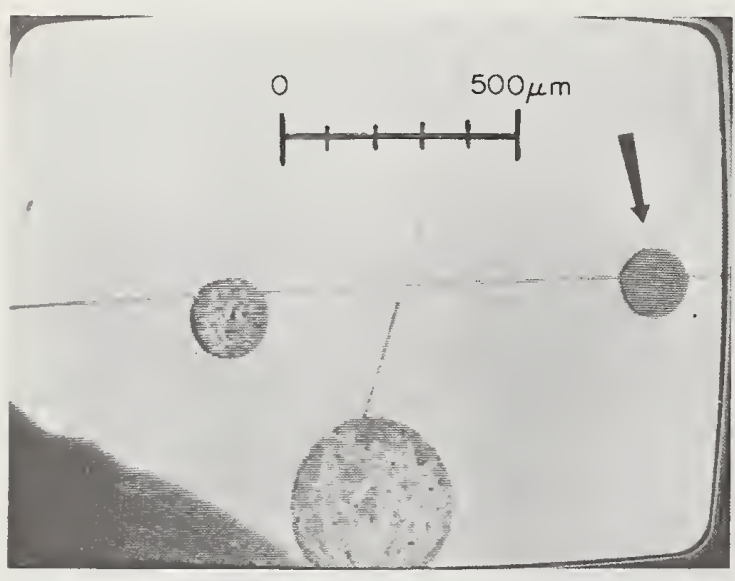

a. particle at 3 o'clock $^{\prime}$

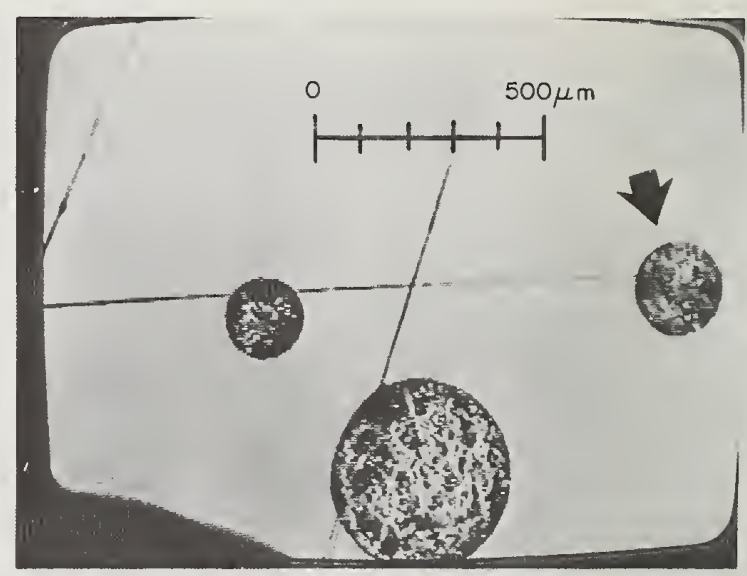

b. expanding slowly

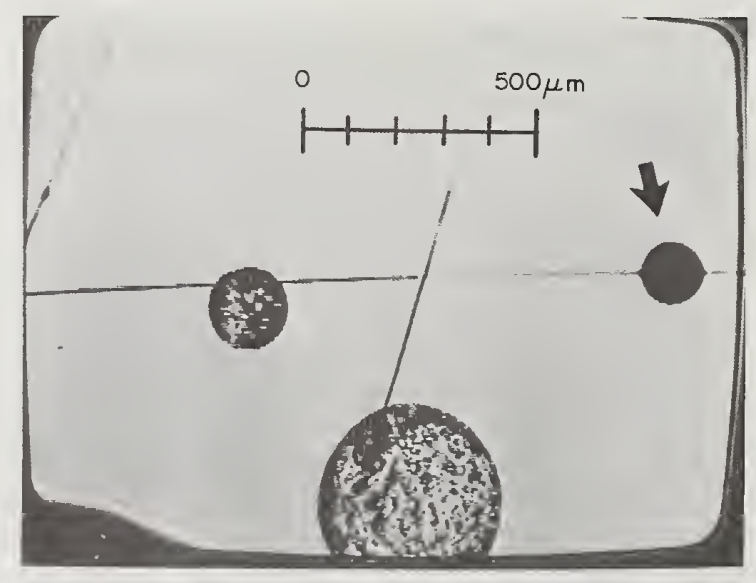

c. collapsed particle

Figure 20. Series of frames showing particle of household oven cleaner spray impacting at 3 o'clock. 
Most oven cleaners shi, dp as large particles--hollow spheres or balloons 100 to 200 micromete; ... size and larger (fig. 21). However, together with these large balloons there is always an abundance of small fine particles in the respirable range as shown impacted on the 2 micrometer strand below the large balloon. They are all very fine--all in the 1-2-3-4-5 micrometer size range. Using the 2 micrometer strand as a reference you can see that they are a 11 certainly under 10 micrometer. They are in the respirable range [5-8].

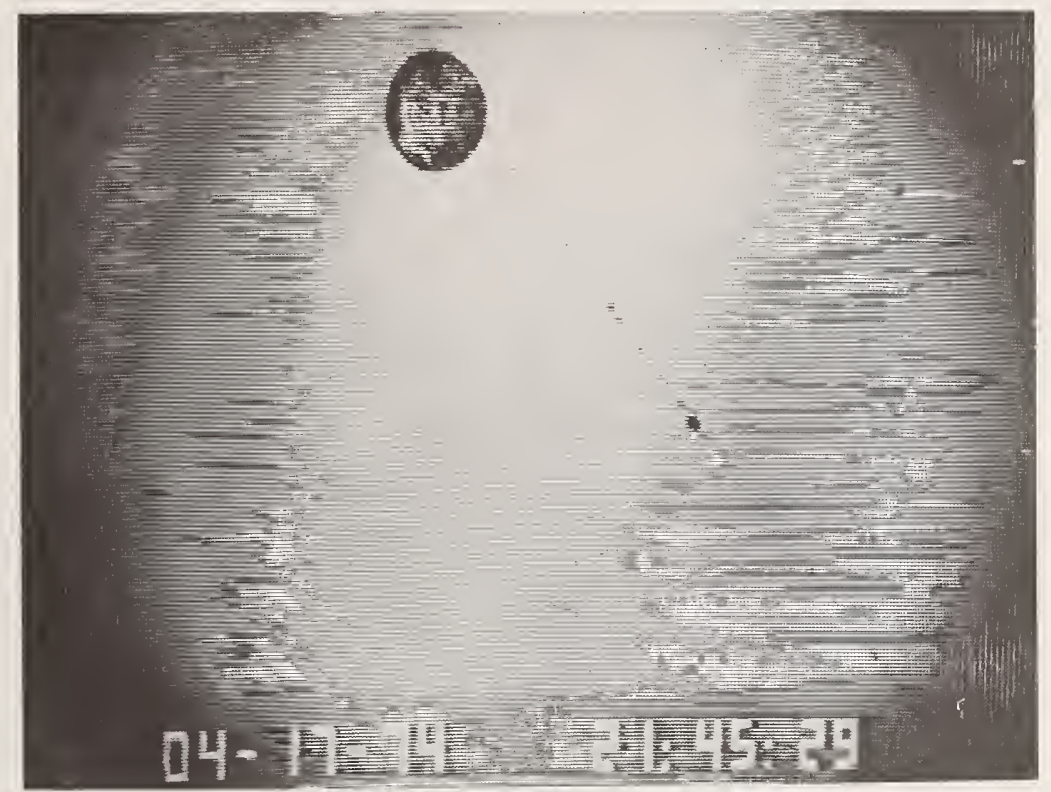

Figure 21. Large particles (oven cleaner).

\section{RESPIRABLE PARTICLES}

Considerable information is available on micro-organisms and their relation to respiratory infections. The amount of particulate material and bacteria retained in the respiratory system is largely dependent upon the size of the inhaled particles. Particles larger than 10 micrometer are efficiently trapped in the upper respiratory tract and are removed by ciliary action or some other means. Particles in the 1 to 10 micrometer range which reach the alveoli are usually retained [5$8]$.

This has been substantiated by a variety of methods and by examination with the Electron Microscope of the lung of humans after death [9$11]$. 
There has been a great deal of work published on this subject. A brief description of some of these diseases follows with references.

- Asbestosis is given as an illustration [12-14].

- The results of breathing coal dust have long been known [15].

- Many aerosols are toxic [11].

- Silicosis is well known [14-16].

- Beryllium has become a problem [17].

- Farmers are now known to suffer from respiratory ailments--farmer's lung [18].

- Those working with sugarcone have been known to develop bagassosis or bagasse worker's lung [19].

- Inhaled cadmium has toxic effects [20-22].

- There are many synergistic effects of inhaled particles [23].

- Various chemicals, if inhaled, have disastrous effects $[24,25]$.

- Cotton workers and those working in cotton textile mi11s [26-28].

- Dust and aerosol inhalation $[29,30]$.

- Certainly cigarette smoking is damaging to the respiratory tract and has been so stated by The Surgeon General [31,32].

- There is an interesting relation between human shock from stress and inhalation [33].

- Finally, the toxic effects of aerosol propellants on the heart is carried in the Archives of Internal Medicine [34].

A further list is included of some other lung diseases that are new to the author. They were discussed in some detail at the Postgraduate Course on Lung Disease, American College of Chest Physicians, Chicago, I11inois, which the author attended, March 19-21, 1973.

- Inhalation of particles of bagasse, the moldy residue of sugarcane found at sugarcane processing plants.

- Farmer's lung and thresher's lung caused from the inhalation of particles formed in moldy hay.

- Mushroom grower's disease.

- Lung damage amongst paprika workers, coffee bean dust, particles from roof thatch, cheese washing processing plants, flour mills, redwood sawdust, cork dust and maple bark.

- Pidgeon breeder's disease caused by breathing airborne particles of dried pidgeon excrement. 


\section{SUSPENDED PARTICLES}

This machine is $270^{\circ}$ useful for studying the trajectory of suspended particles floating in the air. You'll see particles in this sequence falling and reversing their direction (fig. 22). We just saw one at $10^{\prime}$ 'clock--there's another at 12. This sequence is shown on the monotor from the instant replay video recorder. You can see the particles falling and then reversing and going upward again. This shows the trajectory of suspended particles in the air.

Reduction in size due to evaporation may be observed during flight. Also, the actual path of flight is shown and recorded. Hence the actual impaction of insecticide particles on insects may be studied frame by frame.

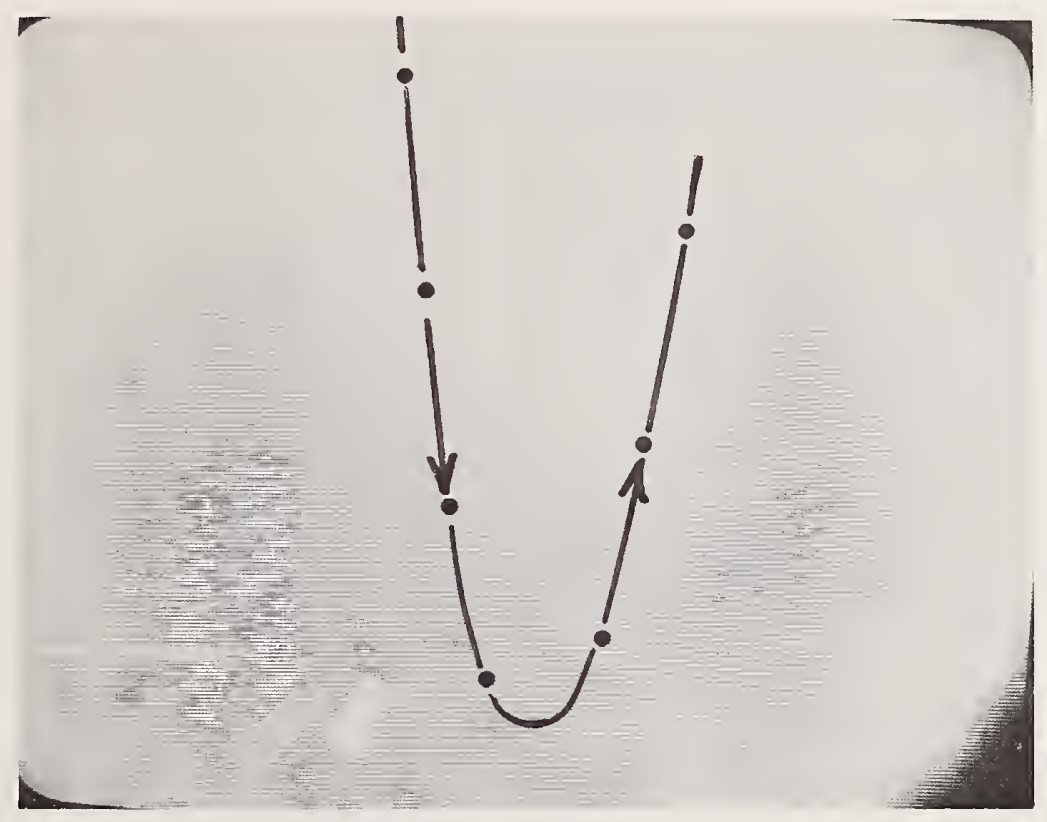

Figure 22. Trajectory of suspended particles in the air.

\section{AGGLOMERATION}

This next sequence will show an agglomeration (fig. 23). If you look at the particles on the right side of the screen at approximately 3 $0^{\prime}$ clock, you'll see them collide with flying particles agglomerating and coming together to form one large blob as shown in the last frame. These frames are 17 milliseconds apart. They were rolled back in slow motion from the Instant Replay Recorder.

This scene shows the three large particles on the right. They are the initial particles and will be impacted as shown in the following sequence. 


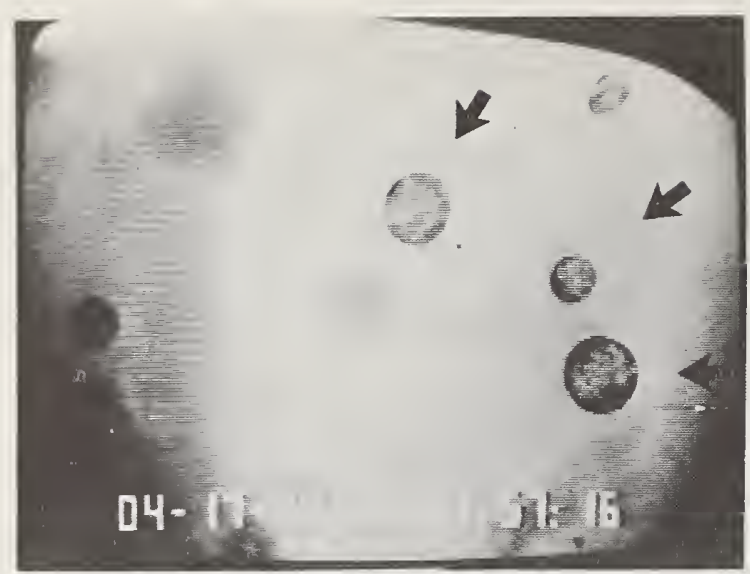

a.

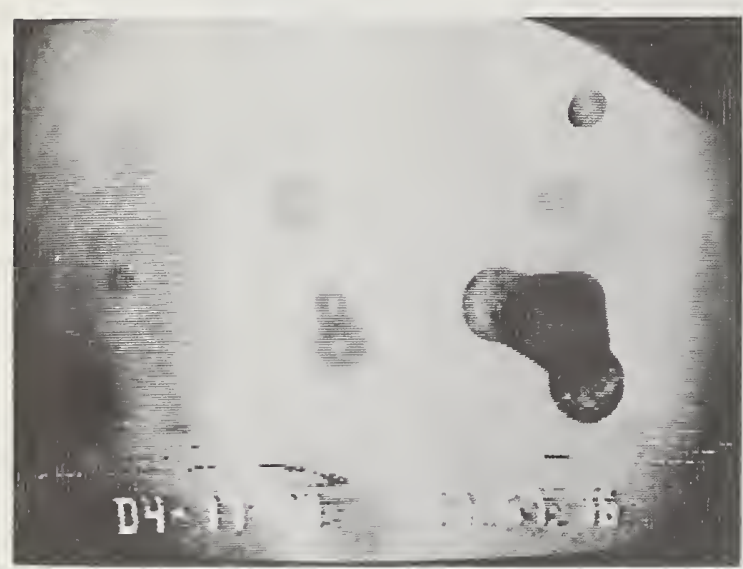

C.

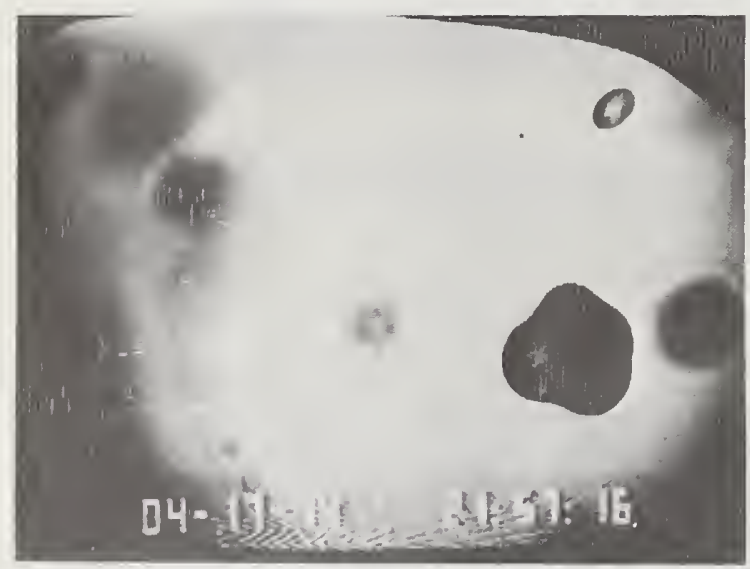

e.

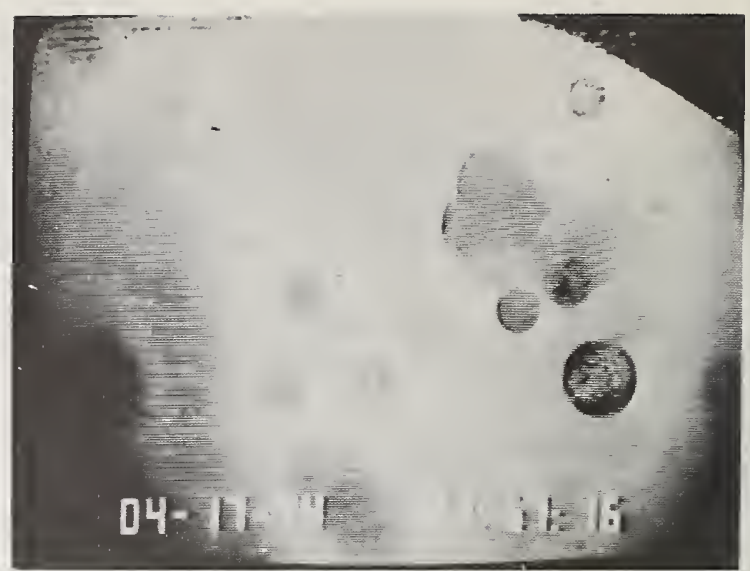

b.

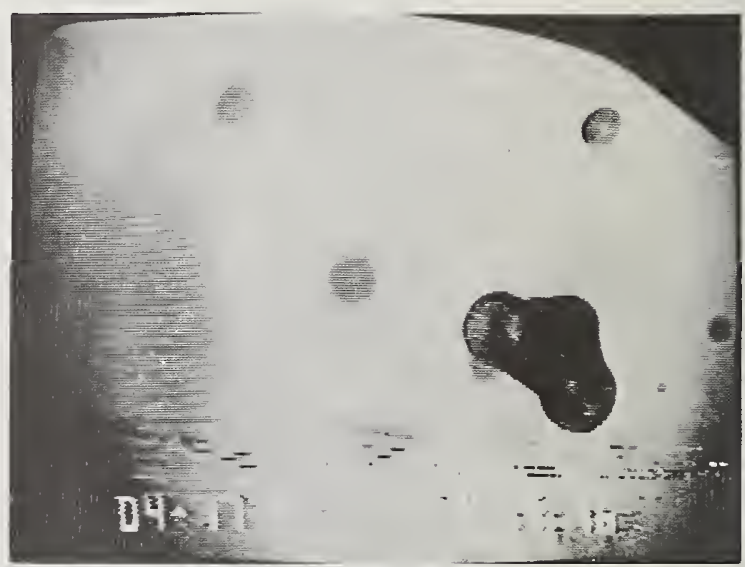

d.

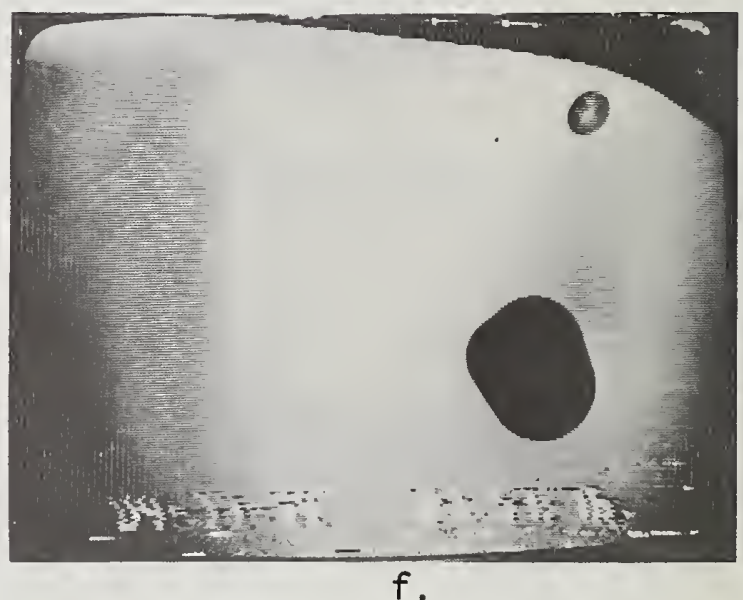

Figure 23. Sequence showing an agglomeration of three large particles (time between frames is 17 militisecond). 
Two additional particles have collided with the top particle. A second particle has appeared inbetween.

They are all solidifying into one conglomerate.

They have pulled together.

And now form one mass.

It is now in the final form. A blob.

\section{INSECTICIDE IMPACTION ON LIVE INSECT.}

The next sequence is a live insect and we're looking at the setal hair protruding from the integument. The insect is on the right and it is alive. The spray is coming from the left and you will see the agglomeration of the small particles of insecticide hitting the tip of that setal hair (fig. 24).

The setal hair is approximately 20 micrometers diameter at its base and 2 micrometers at the very end or at the tip. Now, there are the particles impacting at the tip of the setal hair. The uppermost setal hair you'll see has very small fine particles impacting at the very end of the setal hair. Those particles are impacting at the very tip and are very smal1. They are 1,2 and 3 micrometers in size. The tip of the setal hair is 2 micrometers. You can use that as a reference and

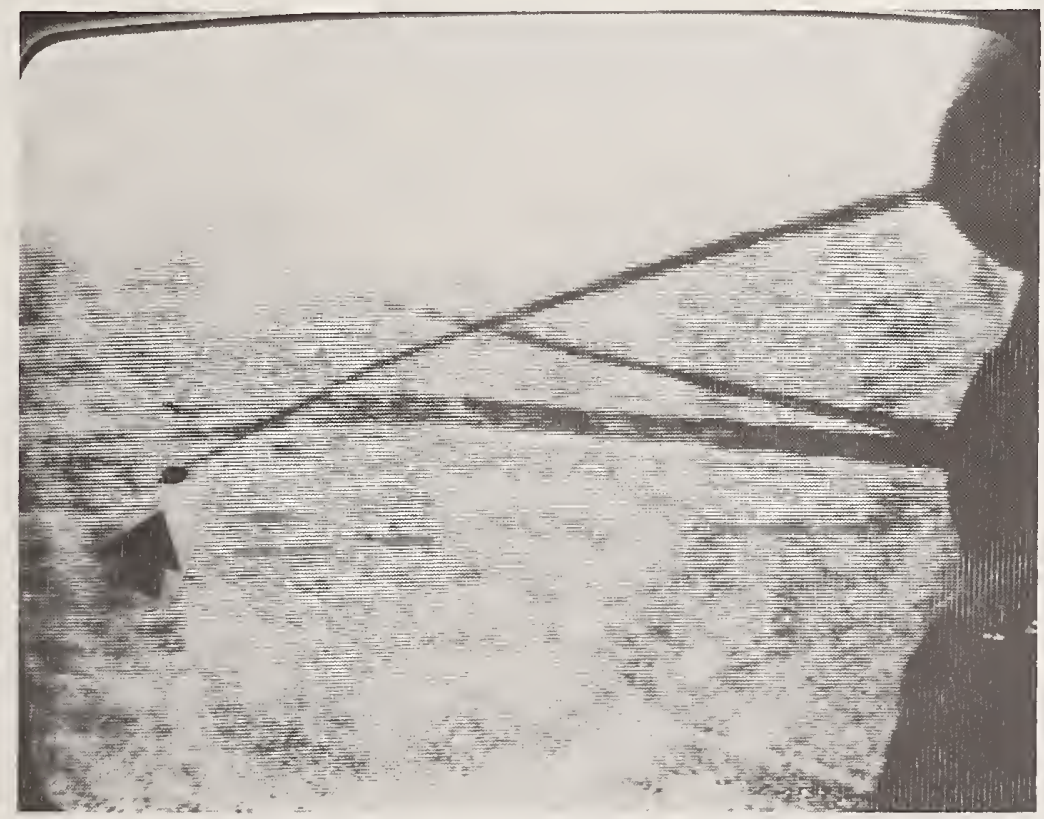

Figure 24. Frame from sequence showing insecticide impaction on live insect. 
get an idea of the size of the particles impacting at the tip. These particles only hit at the tip of the setal hair. They agglomerate at the tip and flow down the setal hair to the skin of integument of the insect. That is the mechanics of an insecticide spray hitting an insect.

\section{CONCLUSION}

This presentation was made via video tape and shown on a TV monitor. In most every case moving particles in flight were displayed and their dynamic activities were shown.

A11 of the photographs were taken from "stills" from the Instant Replay Recorder used in this presentation. The recorder was stopped and the picture was held still on the TV monitor. Polaroids were taken of the held scene. These stills are depicted in this technical report.

SUMMARY

I have shown excerpts from various contracts that I have performed with this machine. The machine presents spray drop pictures in real time. These pictures are stored in an Instant Replay Recorder and may be played back in slow motion frame by frame to study impaction, evaporation, agglomeration, droplet breakup, shape and size distribution.

Size distribution may be obtained in real time by adding the automatic Scanner. Very fast particle counts are realized, i.e., 10,000 counts per second.

The machine is useful for evaluating the size of all aerosols, such as hair sprays, antiperspirants, deodorants, air fresheners, insecticides, asbestos particles, coal cust, atmospheric pollution and the like.

\section{REFERENCES}

[1] Hotham, G. A., "Sizing Aerosols by Laser Holography," Aerosol Division Technical Seminar, Chemical Specialties Manufacturers Association Conference, Washington, D.C., December 4, 1973.

[2] Hotham, G. A., "Particle Sizing by Laser Holography," Society of Experimental Stress Analysis, Los Angeles, Calif., May 14, 1973.

[3] Irani, R. R., and Callis, C. F., "Particle Size: Measurement, Interpretation, and Application," John Wiley \& Sons, Mar. 1963.

[4] Dalla Valle, J. M., "Micrometrics, The Technology of Fine Particles," Pitman \& Sons.

[5] Lourenco, R. V., "Inhaled Aerosols", Archives Internal Medicine, Vol. 131, Jan. 1973. 
[6] Goldberg, I. W., and Lourenco, R. V., "Deposition of Aerosols in Pulmonary Disease," Archives Internal Medicine, Vol. 131, Jan. 1973.

[7] Lourenco, R. V., Klimek, M. F., and Borowski, C. J., "Deposition and Clearance of $2 u$ Particles in the Tracheobronical Tree of Normal Subjects--Smokers and Nonsmokers," Journal of Clinical Investigation, Vol. 50, 1971.

[8] Pierce, A. K., and Sanford, J. P., "Bacterial Contamination of Aerosols," Archives Internal Medicine, Vol. 131, Jan. 1973.

[9] Davis, M. J. G., "Electron-Microscope Studies of Asbestosis in Man and Animals," Annals New York Academy Science, Vol. 132, pp. 98$111,1965$.

[10] United States Department of Health, Education, and Welfare: Occupational Characteristics of Disabled Workers, by Disabling Condition: Disability insurance benefit awards made in 1959-1962 to men under age 65, Washington, D.C. Government Printing Office, 1967 (PHS Publication No. 1531).

[11] Allison, A. C., Harington, J. S., Birbeck, M., et a1., Observations on the cytotoxic action of silica on macrophages, Inhaled Particles and Varpous II. Edited by C. N. Davies, Oxford, Pergamon Press, 1967, pp. 121-131.

[12] Knox, J. E., Holmes, S., Do11, R., and Hil1, I.D., "Mortality from Lung Cancer and Other Causes Among Workers in an Asbestos Textile Factory," British Journal Industrial Medicine, Vol. 25, pp. 293303, 1968.

[13] Biological Effects of Asbestos, Ann. New York Academy of Science, Vol. 132, 1-765, 1965.

[14] Murphy, R. L. H., Asbestosis in pipe coverers engaged in new ship construction. Thesis, Harvard University School of Public Health, Boston, Mass., 1968.

[15] Morgan, W. K. C., "The prevalence of coal workers' pneumoconiosis," Amer. Rev. Resp. Dis., 98, 306-310, 1968.

[16] Trasko, V. M., "Silicosis, a Continuing Problem," Public Health Rep. 73, 839-846, 1958.

[17] Tepper, L. B., Hardy, H. L., Chamberlin, R. I., "Toxicity of Beryllium Compounds," Ams terdam, Elsevier Publishing Company, 1961.

[18] Barbee, R. A., Callies, Q., Dickie, H. A., et al., "The Long-Term Prognosis in Farmer's Lung," Amer. Rev. Resp. Dis., 97, 223-231, 1968. 
[19] Nicholson, D. P., "Bagasse Worker's Lung," Amer. Rev. Resp. Dis., $97,546-560,1968$.

[20] Paterson, J. D., "Studies on the Toxicity of Inhaled Cadmium. III. The Pathology of Cadmium Smoke Poisoning in Man and in Experimental Animals," J. Industr. Hyg. Toxicol., 29, 294-301, 1947.

[21] Spolyar, I. W., Keppler, J. F., Potter, H. G., "Cadmium Poisoning in Industry: Report of Five Cases, Including One Death," J. Industr. Hyg. Toxicol., 26, 232-240, 1944.

[22] Buxton, R. St. J., "Respiratory Function in Man Casting Cadmium Alloys. Part II. The Estimation of the Total Lung Volume, its Subdivisions, and the Mixing Coefficient," British Journal Industrial Medicine, Vol. 13, pp. 36-40, 1956.

[23] Dalhamn, T., Strandberg, L., "Synergism Between Sulphur Dioxide and Carbon Particles: Studies on Adsorption and on Ciliary Movements in the Rabbit Trachea in Vivo," Air Water Pollut., 7, 517-529, 1963.

[24] Peters, J. M., Murphy, R. L. H., Pagnotto, L. D., et a1., "Acute Respiratory Effects in Workers Exposed to Low Levels of Toluene Diisocyanate (TDI)," Archives Environmental Health (Chicago), 16, 642-647, 1968.

[25] Gandevia, G., "Studies of Ventilatory Capacity and Histamine Response During Exposure to Isocyanate Vapour in Polyurethane Foam Manufacture," British Journal Industrial Medicine, 20, 204-209, 1963.

[26] Schrag, P. E., Gullett, A. D., "Byssinosis in Cotton Textile Mi11s," Amer. Rev. Resp. Dis., 101, 497-503, 1970.

[27] Bouhuys, A., Linde11, S. E., "Release of Histamine by Cotton Dust Extracts from Human Lung Tissue in vitro," Experientia, 17, 211$212,1961$.

[28] Bouhuys, A., van de Woestijne, K. P., "Respiratory Mechanics and Dust Exposure in Byssinosis," J. C1in. Invest., 49, 106-118, 1970.

[29] Widdicombe, J. G., Kent, D. C., Nadel, J. A., "Mechanism of Bronchoconstriction During Inhalation of Dust," J. Appl. Physiol., 17, 613-616, 1962.

[30] DuBois, A. B., Dautrebande, L., "Acute Effects of Breathing Inert Dust Particles and of Carbachol Aerosol on the Mechanical Characteristics of the Lungs in Man: Changes in Response after Inhaling Sympathomimetic Aerosols," J. Clin. Invest., 37, 17461755, 1958. 
[31] Bouhuys, A., Schilling, R. S. F., van de Woestijne, K. P., "Cigarette Smoking, Occupational Dust Exposure, and Ventilatory Capacity," Archives Environmental Health (Chicago), 19, 793-797, 1969.

[32] Dieh1, H. S., M.D., "The Surgeon General Reports on the Health Consequences of Smoking," Tobacco and Your Health, 1965, 1967, 1969, and 1972 (Washington, D.C.).

[33] Schayer, R. W., "Relationship of Induced Histidine Decarboxylase Activity and Histamine Synthesis to Shock from Stress and from Endotoxin," American Journal Physiology, 198, 1187-1192, 1960.

[34] Harris, W. S., M.D., "Toxic Effects of Aerosol Propellants on the Heart," Archives Internal Medicine, American Medical Association, (Chicago), Jan. 1973.

\section{DISCUSSION}

ROBERT KNOLLENBERG: I have a question as to your depth of field on a very small particle? One that becomes increasingly small?

HOTHAM: Typically the depth of field for a 100 micrometer particle is plus or minus $2 \mathrm{~mm}$. The optical system has been designed to have a sharp cut-off after this depth of field has been exceeded. So that particles do not gradually drop out of focus but are cut off abruptly; they are either in-focus and appear as black dots or they are out-offocus and are very grey and are not sensed by the computer. Since this is programmed in by contrast setting in the memory holding tube, it abandons all greys and retains only the in-focus black images. This system works on sizing only the in-focus particles.

KNOLLENBERG: How do you make that discrimination?

HOTHAM: The computer logic rejects all particles that show up with diffraction patterns around them, i.e., particles out-of-focus. Hence, in summary, the Automatic Scanner sees only in-focus particles.

Many frames are being stored in the Automatic Scanner. Typically 60 frames or 100 to 1000 frames per second are being stored and scanned so that the system is scanning many, many frames taken within the spray and statistically the in-focus particles counted, several thousand per second, represent a true count and size distribution of the particles in the spray.

LAURENCE DOEMENY: What is the resolution of the instrument? Have you looked at any fibers like asbestos?

HOTHAM: The smallest particles that I have looked at were some polystyrene spheres that were 980 Angstrom in diameter; they were fuzzy but I could detect them. 
We are quoting a minimum resolution of 3000 Angstrom which is 0.3 micrometers.

DOEMENY: Have you looked at asbestos?

HOTHAM: No.

VIRGIL MARPLE: What is the cost of this instrument?

HOTHAM: The laser system is $\$ 25,000$ and the computer that does the automatic scanning is $\$ 60,000$.

RONALD NELSON: How long does it take to scan an image?

HOTHAM: Less than a millisecond. The laser machine is generating video signals which are fed to the computer; each frame containing many hundreds of particle images, is stored. It takes less than a millisecond to scan that stored frame and rack up in the registers the counts in the different size ranges. Realistically you are talking about counts of 10,000 particles per second.

JOHN WEINNEGAR: Have you ever looked at the breath that someone exhales after inhaling an aerosol, perhaps one that's been humidified?

HOTHAM: Yes, tobacco smoke. The smoke was put into a rubber bulb (dry) and then squeezed and put into the path of the laser and the picture showed dry tobacco smoke particles. Secondly, the tobacco smoke was inhaled and blown from the mouth into the laser, and you could see the difference in the size of the particles exhaled. The exhaled particles from the human lung were larger than those expelled from the dry rubber bulb. Hence, the exhaled particles had been humidified while they were in the lung.

REG DAVIES: With your particular system, and where you focus this onto the camera and then onto the screen, can this just be focused, say for example in our case where we have a Quantimet 720 image analyzer with a TV camera, can this be focused through our system?

HOTHAM: I believe your question is, can the Laser Machine be made to work with the Quantimet 720? The answer is "yes." We sell both the Laser Machine and Image Analysis Computers together. They do interface. Thus you accomplish real time size distribution.

DAVIES: When you interface, this computer scanner, which particular scanner do you use? Your own?

HOTHAM: Right now I'm using the Bausch \& Lomb Image Analyzer.

DAVIES: In other words you focus onto the TV scanner to find space, and then use that to size on the screen. 
HOTHAM: Yes, you focus into the spray or select a region of the spray you wish to measure. Those spray images are held in the memory tube and scanned by Image Analyzer or Automatic Scanner as we call it and you get size distribution directly.

ILAN CHABAY: What is the amount of energy you are hitting these particles with per pulse:

HOTHAM: It is 200 microjoules, peak power 2-50 KW, pulse width 10 nanoseconds, 1 to 1000 pulses per second.

I have studied the heart beat of small micro-organisms. The Daphnia is one illustration. It is about a millimeter long. The heart beat was measured under the influence of various narcotics, both stimulants and depressants. The Daphnia was live and continuously receiving the laser. The pulse width is so small, 10 nanoseconds, 10 billionths of a second, that it did no harm and the Daphnia biological specimen swam away quite lively after the exposure.

CHABAY: You said something at some point about trajectory of suspended particles in the air, and I couldn't see the particles.

HOTHAM: Yes, the particles came out quite small on the TV screen, about $1 / 4$ of an inch in diameter and were hard to see.

CHABAY: If I saw it correctly they were merely descending and then rising again.

HOTHAM: This test was performed in an 8-ft high tower or settling chamber. The particles were introduced at the top. Monodispersed particles of 100 micrometers were used.

They fall with gravity until the counter flow of air from the bottom balanced their flight. What you saw on the screen was the floating point for the particles. At this point change in size and shape during evaporation could be studied. It was a study in evaporation to determine the final shape.

These particles were composed of an experimental insecticide. There was some concern by the customer that the insecticide particles would wind up as crystalline particulates in the shape of frisbies. Frisbie particles drift inordinately far, drifting from the intended field to a neighboring crop belonging to another farmer.

In this test it was clearly shown that the particles did not go to frisbies but remained as liquid drops.

Also, in reference to your question about the amount of energy absorbed by aerosols from the laser and the possible effect it might have on them, I would like to illustrate some observations I have made. 
In looking at impacted aerosol liquid drops on those 5 micrometer strands, I have found that if you select one drop and fire the laser slowly, at say, once a minute and look at the drop you will see that it does not change in size. But most important, when you speed up the firing rate of the laser to say 100 pulses per second, also, the liquid drop did not show any change in shape or size.

You can probably reason through this observation by remembering that the laser beam cross-section is quite large, several millimeters when it reaches the small particle, which in this case was 10 micrometers, so that the beam is spread out sufficiently not to cause damage to the aerosol.

CHARLES PARMENTER: Have you tried doping the liquid particles with fluorescent dyes and looking at it by fluorescence?

HOTHAM: Yes, we have done that. The fluorescent particles change the way the insecticide droplet acts. If you mix the fluorescent particles with the insecticide, it will change the density and character of the droplet. These insecticide droplets with added fluorescent particles do not impact on setal hairs with the same efficiency as do unmixed particles. I am only familiar with droplets of insecticide in the 1 to 20 micrometer size range that had added to them fluorescent particles in the 1 to 5 micrometer range.

You are probably suggesting the use of the fluorescent dyes because I am using a UV laser of considerable power hoping to use the fluorescent effect to detect the size of the particles. Unfortunately the Laser Machine as it is now constructed forms images of the particles only; it does not sense light level or the fluorescent effect. The machine could be made to sense light level by simply using a photodetector.

EDGAR ETZ: At this point do you prefer to size the particles in flight or after they have impacted on a target strand?

HOTHAM: You can do it both ways. The short pulse of the laser, 10 nanoseconds, makes a stop action picture of the flying aerosol particle. This frozen picture of the particle is sized by eye by the reticule on the face of the TV monitor. Or the frame may be stored in the memory tube and sized automatically by the Automatic Scanner.

ETZ: How do they compare? What happens to the particle once it makes contact with a surface? And depending on the nature of the surface, doesn't that surface change the characteristics of the aerosol? Is it reliable to size a particle of a liquid aerosol when it is on a surface?

HOTHAM: If you wish to obtain a true size distribution of the spray in air as generated from a dispenser, then you should record the particles in free flight. The Laser Machine is designed to operate this 
way. It photographs the particles in free flight. The images are sized to provide size distribution, either manually or by the Automatic Scanner.

The particles in this case are in free flight and have not been impacted.

In the case of the hair spray, I showed you particles hitting target strands to simulate the hitting of human hair. Those pictures showed the actual mechanics of impaction. This was also shown for a deodorant (liquid) and an antiperspirant (particulate). I wanted to show the actual mechanics of impaction.

Certainly, to answer your question, a particle hitting a flat surface is spread out and alters the shape of the aerosol. This Laser Machine technique does not use impaction on flat surfaces to determine size distribution.

Going back to the hair spray and the measurement in free flight-just as a reminder, the laser is fired through the spray perpendicular to the axis of the spray. If you fire the laser through the axis of the spray at say 8 inches downstream, you will get a different size distribution from a measurement taken at say 12 inches downstream. Also, measurements made on the outer edges of a spray will be entirely different from those taken along the center line or axis of the spray.

In the case of a hair spray, you want to produce fairly large drops in the 150 to 300 micrometer size range or larger. Once the hair has been styled by a beautician, the object of the spray is to hold the hair in that position. You do not want the spray to bring the hair fibers together and wet them into a mat. The droplet ideally will strike two hairs simultaneously, straddle them, evaporate and leave a supporting residue to space the hairs apart. The other effect is for the droplet to spread along the length of the hair fiber and around it, and evaporate, giving it a strong sleeve support to maintain its form. Again, it is the "shellac-like" residue that gives hair its support.

Unfortunately, accompanying these large drops in a hair spray are a great abundance of fine, small respirable particles in the 1 to 10 micrometer size range which are formed as satellites to the larger drops which are formed, and which if inhaled remain in the lung for long periods of time.

Ideally, then, you wish to produce a spray with many large drops for a hair spray without the generation of a lot of respirable particles.

PETER PELLA: Is there any relationship between these and antiperspirants? I'm thinking of aluminum chloride that is generally put in antiperspirants. Do you see a residue of that? 
Hotham: In a hair spray you want the liquid drop to hit the hair and leave a bonding or supporting residue.

In an antiperspirant you want most of the liquid to have evaporated just before hitting the skin so that it won't feel cold, i.e., from evaporation on the skin after impacting. But you want the particle to be sufficiently wet to give some sticking qualities to the skin.

For best efficacy you would want the greatest surface area on the drops per gram of antiperspirant expelled from the dispenser. Hence the antiperspirant is usually generated as very small fine particles. This produces the greatest antiperspirant effect and has the greatest efficacy.

Unfortunately, these small particles, if they get down into the lower lung, into the alveoli, they usually remain.

To answer your question about seeing aluminum chloride in an antiperspirant. Yes, you can see the residue at impaction. That is what I showed in the film on antiperspirants. You were shown the residue on the 5 micrometer strands. You were also shown flying particles of antiperspirant that were round, liquid drops. At impaction, or shortly after (microseconds or milliseconds later), the impacted particles completed their evaporation and hung onto the strand as residues. You can see this from their jagged and irregular shape. That was the residue of aluminum chloride. This has been substantiated by chemical analysis of the residue.

On the other hand, a deodorant flies as a liquid drop, impacts as a liquid drop and remains in the liquid form to spread over the skin. Particles of deodorant in the 1 to 10 micrometer size range were shown to you today just after the series on antiperspirants. 


\section{RAPID RESPIRABLE MASS MEASUREMENT}

Lawrence Doemeny, George Carson and Bruce Almich U.S. Department of Health, Education, and Welfare Public Health Service Center for Disease Control National Institute for Occupational Safety and Health Engineering Branch

Division of Laboratories and Criteria Development 1014 Broadway

Cincinnati, Ohio 45202

\section{ABSTRACT}

The respirable mass concept of dust sampling will be used to introduce the GCA Corporation, beta absorption particulate aerosol sampler. NIOSH has extensively evaluated the GCA instrument as a coal dust monitor and is beginning to apply it to the sampling of other aerosols. The evaluation included mass loading, accuracy and environmental simulations. Other sampling methods which look promising will be discussed.

Key words: aerosol size measurements; beta absorption; cascade impactor; coal dust monitor; dust inhalation hazards; environmental sampler; respirable dust sampler.

\section{RESPIRABLE MASS CONCEPT}

Airborne particles having aerodynamic diameters less than $100 \mu \mathrm{m}$ present a potential inhalation hazard to the worker. The nature and magnitude of the hazard depends upon the number of particles within a size range and primary mode of entry to critical organs. The fraction of inhaled articles with aerodynamic diameters greater than $10 \mu \mathrm{m}$ impact or settle on the nasal and oral passages and are eliminated through the gastrointestinal tract. The hazard from these and other particles depend upon their physical, chemical and biological properties. Generally, the fraction of particles less than $10 \mu \mathrm{m}$ present the greater potential health risk and this fraction has a probability of depositing on the nonciliated passages of the respiratory system.

The deposition of inhaled particles has been studied theoretically and experimentally. The currently accepted lung regional deposition model is that arrived at by a special Task Group on Lung Dynamics to the International Commission of Radiological Protection (ICRP) Committee 2 [1]. The ICRP model is based on a theoretical treatment using log- 
normal dust distributions, and an anatomical model at several ventilatory capacities.

Today several criteria and definitions exist for respirable dust. Common to these definitions is the concept that the particles be sized according to their aerodynamic diameter. These organizations mentioned below have different criteria for respirable dust sampling. The criteria and size selective samples used to collect representative respirable dust levels are based on the lung depositions model. Figure 1 shows the criteria used by each organization and also the ICRP lung deposition model. The British Medical Research Council (BMRC) curve is the particle penetration resulting when a stacked parallel plate horizontal-elutraitor type sampler is used. This curve is theoretically derived and is based on the gravimetric settling of particles. The BMRC is the respirable mass fraction criteria used by England and the United States when sampling coal dust in mines.

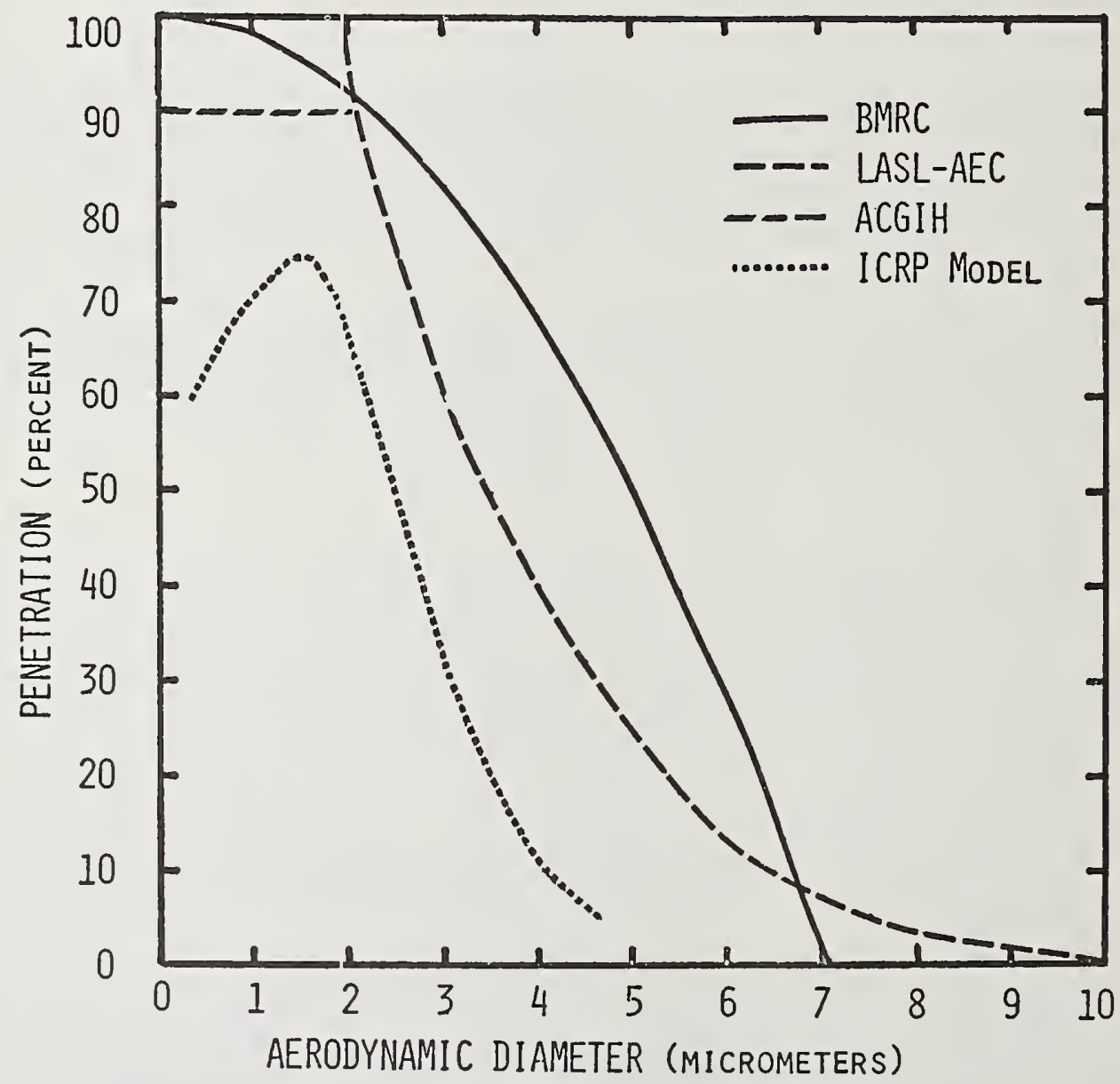

Figure 1. Lung deposition model and respirable mass definitions. 
The Los Alamos Scientific Laboratory (LASL-AEC) and the American Conference of Governmental Industrial Hygienists (ACGIH) curves are identical except for particles with aerodynamic diameters less than 2 $\mu \mathrm{m}$. Although the curves were developed from lung depositions studies and not devised with size selective dust samplers in mind, small cyclone inertial collectros closely approximate the curves. In the United States the ACGIH curve is used as the respirable dust definition for all mineral dusts (coal dust excepted) and would presumably apply to organic particulate aerosols.

NIOSH and OSHA have favored evaluating dust exposure using portable sampling equipment worn by the individual to obtain an eight-hour timeweighted average (TWA) breathing zone sample. These samplers include a battery operated pump and a sampling head consisting of a particulate classifier and preweighed membrane filter. The most commonly used particulate classifier is a $10-\mathrm{mm}$ nylon cyclone which separates particles aerodynamically according to the ACGIH criteria when operated at $1.7 \mathrm{lpm}$. Particles passing through the cyclone are collected on a filter and weighed or could be chemically analyzed as the respirable fraction.

NIOSH is interested in occupational aerosol spray exposures in occupations where they are manufactured or used such as in beauty schools and salons, spray painting, cleaning services, etc. A specific pulmonary disease, thesaurosis, has been reported in the hair dressor occupation and NIOSH has conducted some investigation of this problem.

These aerosol sprays present a unique problem to aerosol sampling because particulate concentrations are extremely high and their physical and chemical properties are unstable. The aerosol is a multicomponent system consisting in part of a propellent, a volatile solvent, a fragrance and an active ingredient. Because of the volatility of some components the aerodynamic diameter of the aerosol and its composition is changing with time. For OSHA compliance this is a staggering problem because an eight hour TWA exposure is the hazard evaluation criteria but an instrument capable of short rapid sampling is required to collect and evaluate the sample with time dependent characteristics. Frequent short-term samples might then be representative of an eight-hour TWA exposure.

\section{AN INSTRUMENT WHICH MAY BE CAPABLE OF MEASURING AEROSOL SPRAY EXPOSURE}

NIOSH has recognized the need for instruments which, al though not necessarily able to measure occupational compliance, or noncompliance can quickly identify areas of greatest hazards concentration so compliance monitoring can be efficiently initiated. Under a NIOSH contract the GCA Corporation has developed a portable, short-term respirable dust monitor for application in "walk-thru" surveys in coal mines. Although developed for coal dust sampling its use is not limited to coal dust. 
GCA (fig. 2) now has available one instrument which can be obtained in one of four configurations (table 1). They are called respirable dust monitors, RDM-101. Depending on the model the RDM's can evaluate respirable dust concentrations ranging from 0.06 to $200 \mathrm{mg} / \mathrm{m}^{3}$. The range change is accomplished by altering the sampling times from 12 seconds up to 8 minutes. The RDM-101 is designed for short time, on the spot measurements of mass concentrations of the respirable fraction of the dust or, with the cyclone preclassifier removed, the total mass concentrations using the principle of beta-particle absorption. With the cyclone removed the impactor is limited to the collection of particulates with aerodynamic diameters less than $50 \mu \mathrm{m}$.

The cyclone and impactor combination comprise a two stage collection system. The first stage is the cyclone. The second stage consists of the inlet nozzle impactor beta absorption assembly and mylar impaction disk. The beta particle source is housed in the impactor nozzle. As material impacts on the mylar disk, radiation is increasingly absorbed, decreasing the radiation reaching the Geiger tube beneath the disk. The transmission of beta particles depends almost entirely on the mass of material per unit area of absorber and the maximum

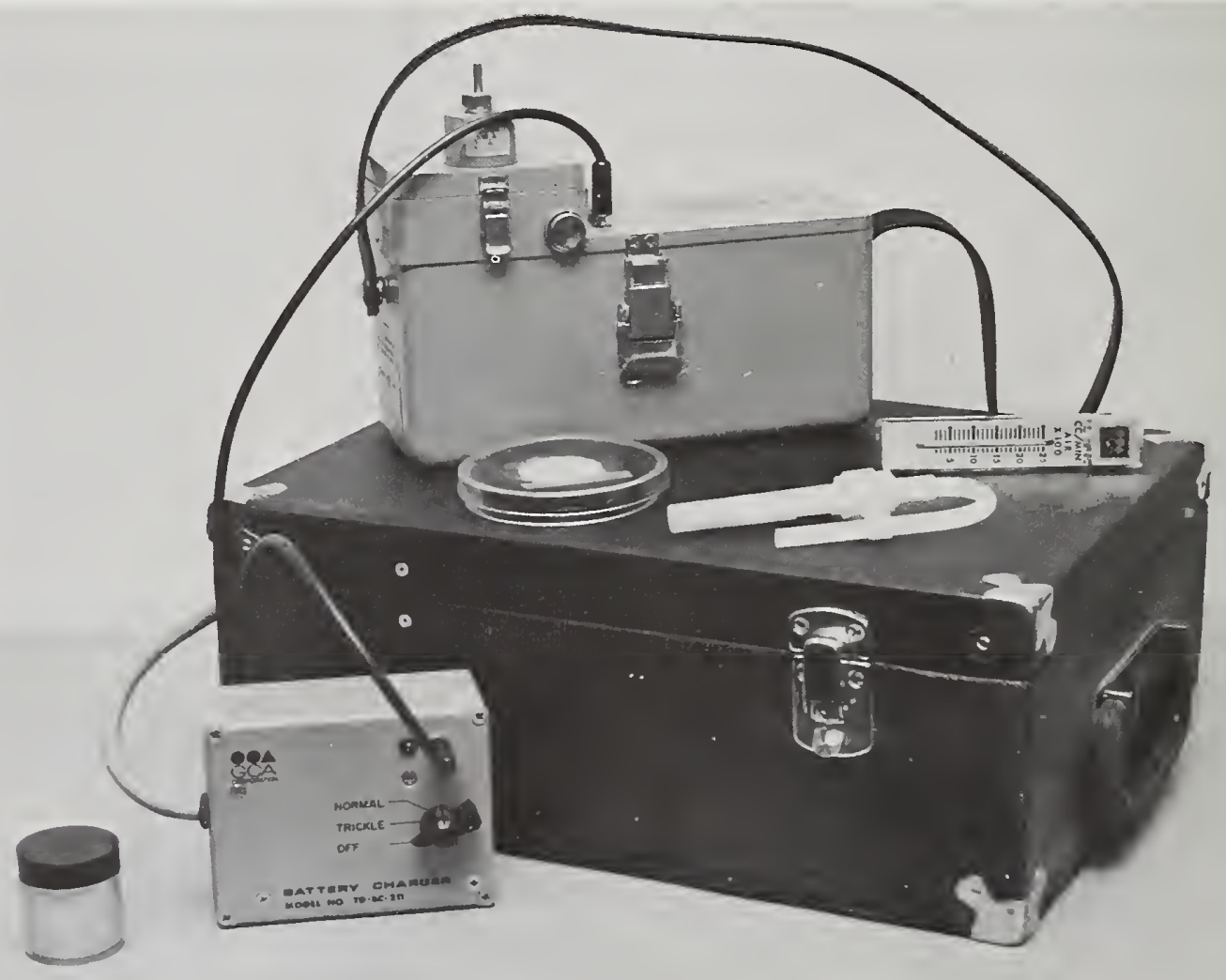

Figure 2. Respirable dust monitor with 10-minute nylon cyclone, battery, charger, air flow calibration ratementer and carrying case. 
Table 1. Respirable dust monitors.

\begin{tabular}{|c|c|c|}
\hline Model & Mass Range & Sampling Time \\
\hline RDM-101-0.2 & 10 to $200 \mathrm{mg} / \mathrm{m}^{3}$ & $12 \mathrm{~s}$ \\
\hline RDM-101-1 & 1 to $50 \mathrm{mg} / \mathrm{m}^{3}$ & $1 \mathrm{~min}$ \\
\hline RDM-101-4 & $8 \mathrm{mg} / \mathrm{m}^{3}$ & $4 \mathrm{~min}$ \\
\hline RDM-101-8 & 0.06 to & $8 \mathrm{~min}$ \\
\hline
\end{tabular}

beta energy. For coal dust, composition has little effect because the beta particle absorption cross section is proportional to the ratio of atomic number $(Z)$ to gram molecular weight $(A)$. For large organic molecules containing mostly carbon, hydrogen, nitrogen, oxygen and some sulfur and phosphorus the error should be negligible. For methane particles (if they were to exist) the instrument would read 25 percent high when calibrated for coal. Similarly, for hexane the RDM would read 16 percent high and as the ratio of carbon mass to hydrogen mass decreases, the error decreases. tion:

Beta radiation is attenuated exponentially according to the equa-

$$
\mathrm{N} / \mathrm{No}_{0}=\exp (-\mu \mathrm{s})
$$

where:

$\mathrm{N}$ and No are the initial and final beta counts produced by the Geiger tube respectively.

$\mu$ is the beta absorption coefficient.

$s$ is average mass of material per unit area.

The mass concentration $C$ is equal to:

$$
C=\frac{A}{\mu Q t}(\ln \text { No }-\ln N)
$$

where:

$A$ is the dust collection area.

$Q$ is the air flow.

$t$ is the effective sampling time. 
The term $\mathrm{A} / \mu \mathrm{Qt}$ is constant set during calibration. The RDM's are completely automatic. By starting the sampling cycle the instrument concurrently begins sampling the atmosphere and takes an initial beta count. During the last few seconds of the cycle the final beta intensity is measured. The difference in the log of the two beta counts is multiplied by the instrument calibration constant and displayed on a three digit LED in $\mathrm{mg} / \mathrm{m}^{3}$ for ten seconds. The value can be recalled any time before the next cycle. The instrument is completely solid state and battery operated. Our tests [2] have confirmed the measurement accuracy to better than \pm 25 percent ( $95 \%$ coefficient of variation) for coal dust. For the RDM-101 the beta counts are taken during the initial and final 20 seconds of the sample period. This value was selected by minimizing the variance in the concentration with respect to the counting time and varies accordingly for each instrument model.

The beta absorption principle has been shown to be useful as an aerosol mass monitor by GCA's instrument and as a stack emission monitor in an instrument sold by Lear-Seigler. The principle can be applied to existing particulate samplers like the cascade impactors which classify particulates into several aerodynamic diameter intervals. Such a unit could then be calibrated to directly display total collected mass, aerodynamic mass median diameter and geometric standard deviation, for an assumed log-normal distribution.

\section{FEW EXPERIMENTS WITH AEROSOL HAIR SPRAY}

Hair spray was directed at an RDM-101 from a distance of $60 \mathrm{~cm}$ for the entire period of the cycle. Masses of approximately $10 \mathrm{mg} / \mathrm{m}^{3}$ and 5 $\mathrm{mg} / \mathrm{m}^{3}$ were obtained for "total" and respirable fractions respectively. As mentioned above, the "total" sample is for aerosols with aerodynamic diameters less than $50 \mu \mathrm{m}$. Literature values show respirable fractions ranging from 0.5 percent up to 65 percent. Associated with the 65 percent respirable mass fraction were observed particles ranging from 1 to $3 \mu \mathrm{m}$ [3] while the 0.5 percent value observed 50 percent of the particles with diameters greater than $35 \mu \mathrm{m}$ [4]. It is difficult to assess the conflicts in this data for the size distribution can vary between products tested. However, the data does show a need for instrumentation which is capable of rapid aerosol measurement to eliminate any ambiguity or doubt in aerosol sizing.

\section{CONCLUSION}

The principle of beta absorption as applied to aerosol mass measurements is viable for sampling high concentrations of aerosols. The detection principle can be applied to existing aerosol sampling equipment like Lundgren, Andersen and May type impactors for rapid size determination by positioning a beta source and detector at each stage of separation. 
Another technique worth mentioning is a new concept in aerosol sizing described by Dr. Lawrence Hrubesh [5]. Dr. Hrubesh passes particles through a tuned microwave cavity. The resonant frequency of the cavity is changed by the presence of the particle. The change in frequency as measured on a spectrum analyzer is proportional to the volume of the particle (eq 3)

$$
\Delta f=\frac{6 f_{0} v\left(\varepsilon^{\prime}-1\right)}{a b d\left(\varepsilon^{\prime}+2\right)}
$$

where:

$f_{0}$ is the resonant frequency of the cavity

$V$ is the volume of the sphere

$\varepsilon^{\prime}$ is the complex permittivity of the dielectric (assumed lossless)

$a, b$ and $d$ are the cavity dimensions

$\Delta f$ is the change in resonant frequency of the cavity by the presence of the sphere.

The instrument in its present form is limited to particles greater than $50 \mu \mathrm{m}$. Dr. Hrubesh and I will meet this summer to discuss methods to extend the usefulness of the device into the respirable range.

\section{REFERENCES}

[1] Task Group on Lung Dynamics to the ICRP, Committee 2. Deposition and Retention Models for Internal Dosimetry of the Human Respiratory Tract, Health Phys. 12, 173 (1966).

[2] Almich, B. P., Solomon, M., and Carson, G. A., "A Theoretical and Laboratory Evaluation of a Portable Direct Reading Particulate Mass Concentration Instrument." In final review before submission to the American Industrial Hygiene Association Journal for publication. Presented at the May 1974 meeting of the American Industrial Hygiene Association, Miami Beach, Fla.

[3] Draize, G. H., Nelson, A. A., Newburger, S. H., and Kelley, E. A., "Inhalation Toxicity Studies of Six Types of Aerosol Hair Sprays." Proceedings of the Scientific Section of the Toilet Goods Association, Number 31, May 1959. 
[4] Brunner, M. J., Giovacchini, R. P., Wyatt, J. P., Dunlap, F. E., and Calandra, J. C. Pulmonary Disease and Hair Spray Polymers: A Disputed Relationship. The Journal of the American Medical Association, 184, 851-857 (June 15, 1963).

[5] Hrubesh, L. W., A Simplified Microwave Instrument for Measuring Particle Volumes and Densities, Rev. Sci. Inst. 45, 125-126 (Jan. 1974).

*Mention of company or product names is not to be considered as an endorsement by NIOSH.

\section{DISCUSSION}

REG DAVIES: When you mentioned that you do this by measuring the respirable percentage of hair sprays or consumer products, are you relating the mass to the residue or to the droplet itself?

DOEMENY: The study was an introductory effort, therefore the results should not be considered conclusive. We were attempting to get an idea as to what percentage of total aerosol was respirable. At the time it would be difficult to say how much solvent evaporated from the sample collected on the impaction disk.

DAVIES: So this figure of one-half percent to sixty-five percent where you say you agree with the literature, is it based purely on the residue or would it have had a little liquid in it?

DOEMENY: As in the previous answer, it is difficult to say what fraction of solvent had evaporated. The presence of solvent in the droplet does influence the droplet aerodynamic diameter. As solvent evaporates the aerodynamic diameter changes. Because the aerosol piles up on the impaction disk, evaporation would be slowed down. The instrument reading would then include the solvent fraction if it were present.

DAVIES: Therefore, you can be over estimating what is respirable or inhalative because you are looking at something that is smaller?

DOEMENY: That is possible, but the literature value of 0.5 percent seems too low. Remember, also, our samples were collected some distance from the aerosol source so that the large particles may never have reached the meter.

GEOFFREY HOTHAM: The sampling in that machine for the hair spray, that's just airborne particles that you are sucking into the machine?

DOEMENY: Correct. 
HOTHAM: What about the big particles that you get in the hair spray, the 200 to 300 micrometer range?

DOEMENY: The instrument samples for particles in the range of 0.4 to $50 \mathrm{\mu m}$. The larger particles are not collected.

HOTHAM: Hair spray contains particles of 150 to 300 micrometers?

DOEMENY: As far as we know that is correct. However we are ultimately interested in the respirable mass fraction which would generally be those less than $10 \mu \mathrm{m}$ in diameter.

HOTHAM: When you make the measurements though, using the fraction by mass, it's quite different than what you quoted.

DOEMENY: Yes. Under this setup particles larger than $50 \mu \mathrm{m}$ are neglected and would then make my earlier statement on respirable mass fraction appear high. machine.

HOTHAM: Because of the large particles that don't get into that

DOEMENY: That is correct.

EDGAR ETZ: How did you get the respirable fraction? Did you run it with and without the cyclone?

DOEMENY: Yes.

ETZ: I'm trying to visualize your sampling situation. Whatever concentration of airborne particles that you have in mind, you are going to force a known volume of this atmosphere into an intake of your sampling pump. Is there anything that goes on in the process of sampling or channeling particles into the intake of your sampling device that causes particles to agglomerate?

DOEMENY: I do not know of any definitive studies but it would seem unlikely that agglomeration occurs in the cyclone sampler for the concentrations, sizes and distributions frequently encountered.

ETZ: Does anyone else in the audience have any feeling for what happens to solid airborne particulates as they are channeled into a small orifice?

DOEMENY: We have done concentration studies of cyclones with aerosol concentration in the $2 \mathrm{mg} / \mathrm{m}^{3}$ and found no effect on the concentration measurement.

ETZ: With varying concentrations at the orifice, what effect does this have? 
HOTHAM: I would like to answer your question from the work I've done with the pulsing laser system. Sampling devices that carry in the airborn particulates, suck in only the small particles. The small orifice pulls in only the small particles and misses the large ones. The size distributions that you obtain from such a machine are therefore distorted.

DOEMENY: With this instrument that is indeed possible. However in most cases, especially for dry, stable aerosols, the fraction is obtained from the cyclone classified sample and a total aerosol sample that has been collected isokinetically on an open face filter.

HOTHAM: Didn't you say the size range only went up to 50 micrometers?

DOEMENY: I was speaking to other methods to obtain respirable mass fractions, like the personal cyclone sampler a miner might wear.

HOTHAM: I'm saying the sampling leaves a little bit to be desired in that the sampling method only takes in the small particles.

DOEMENY (added in proof): This is not correct. The cyclone was designed to sample respirable aerosols which it collects very well. The larger particles are collected on the open face filter.

ETZ: I would think that depending on the dryness of the atmosphere, these particles are charged and when they increase their concentration you increase the chances of agglomeration.

DOEMENY: You don't increase their concentration by passing them through the cyclone. 


\title{
PARTICULATE MASS MEASUREMENT BY PIEZOELECTRIC CRYSTAL
}

\author{
Raymond L. Chuan \\ Celesco Industries, Inc. \\ Costa Mesa, CA 92626
}

\section{ABSTRACT}

An adhesive-coated quartz crystal working as the impaction surface capturing particulates from decelerating jets measures mass by frequency change, to a sensitivity of $10^{9} \mathrm{hz} \mathrm{gm}^{-1}$. The scheme is applied to a number of working instruments which measure continuously and directly particulate mass concentration, and particulate mass distribution (by using the devices in a cascade).

Key words: aerosol sizing; cascade impactor; particle detection by impaction; particle size measurements, piezoelectric crystal; quartz crystal microscope.

\section{DESCRIPTION OF THE TECHNIQUE}

The Quartz Crystal Microbalance (QCM) is a piezoelectric device which senses the mass of particulate matter impacted aerodynamically on an adhesive-coated quartz crystal through a number of small jets and creates a decrease in the resonant frequency of the crystal. Two crystals are placed close together in the Sensing Module, one acting as the impactor surface (The Sensing Crystal) and the other acting as the Reference Crystal (and temperature compensator). See figure 1. The reference oscillator is set at a slightly higher frequency than that of the Sensing 0scillator (both operating near $10^{7} \mathrm{~Hz}$ ) so that a beat frequency of about $10^{3} \mathrm{~Hz}$ is produced in the mixer. A decrease in the sensing frequency (due to a mass increase) then results in an increase in the beat frequency.

The fundamental relationship between mass change and frequency change for a nominal frequency of $10^{7} \mathrm{~Hz}$ is (from ref. 1):

$$
\Delta f=2.2 \times 10^{8} \frac{\Delta m}{A}
$$

in which $\Delta f$ is the frequency change in $\mathrm{Hz}, \Delta \mathrm{m}$ the mass change in gram and $A$ the active area in $\mathrm{cm}^{2}$ on the crystal where mass is added. 


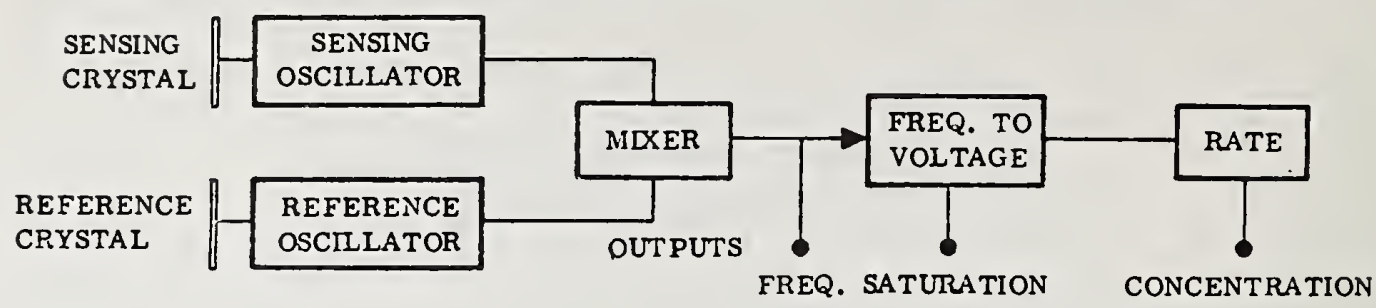

ELECTRONIC SCHEMATIC

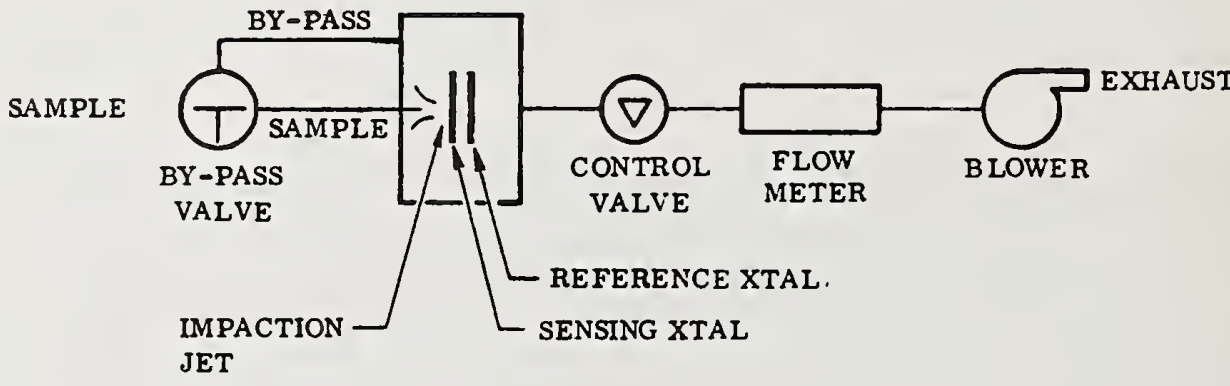

$73-220$ JET

Figure 1. Sample air flow and electrical schematic.

Typically, the active area is about $0.4 \mathrm{~cm}^{2}$, resulting then in

$$
\frac{\Delta f}{\Delta m}=5.5 \times 10^{8} \mathrm{~Hz} / \mathrm{g}
$$

Thus, a nanogram of mass addition will give rise to a frequency change of about $0.5 \mathrm{~Hz}$. The beat-frequency from the mixer is amplified and converted to a voltage in the frequency-voltage-converter whose output is proportional to cumulated mass. The rate of change of the beat-frequency, for a fixed volume flow rate of sampling, is proportional to the particulate mass concentration. The output of the frequency-voltage converter is, therefore, differentiated in the rate converter whose voltage output is then the mass concentration.

\section{I . DISCRETE MASS MEASUREMENT}

An early form of the instrument based on the QCM technique was reported in references 2 and 3 in which the ability of the device to measure the mass of discrete particulates was discussed. If the number density of particles in suspension in air is small enough so that the impactions of particles against the crystal are not coincident, the individual masses can be measured. 
The arrival of a particulate mass on the crystal results in a stepwise rise in the beat frequency. If the beat frequency is converted to an analog voltage and then differentiated, the step-wise frequency rise becomes a pulse whose heights is proportional to the mass of the particulate.

With conventional circuitry it is possible to resolve a frequency change, over a short time interval, of the order of $10^{-2} \mathrm{~Hz}$, equivalent to a mass of about $2 \times 10^{11} \mathrm{~g}$. Actual test results to date have reached a mass resolution of about $1 \times 10^{-11} \mathrm{~g}$. This is the mass of an equivalent sphere of diameter 2 microns with a mass density of $2 \mathrm{~g} / \mathrm{cm}^{3}$.

Figure 2 shows an actual recording trace of a differentiated analog signal from the beat frequency change. The five readily discernible pulses represent discrete masses of from $5 \times 10^{-11}$ to $6 \times 10^{-10} \mathrm{~g}$. These are produced by particulates of mass density $3 \mathrm{~g} / \mathrm{cm}^{3}$; thus the largest pulse represents a single particulate of diameter 7.5 micrometers.

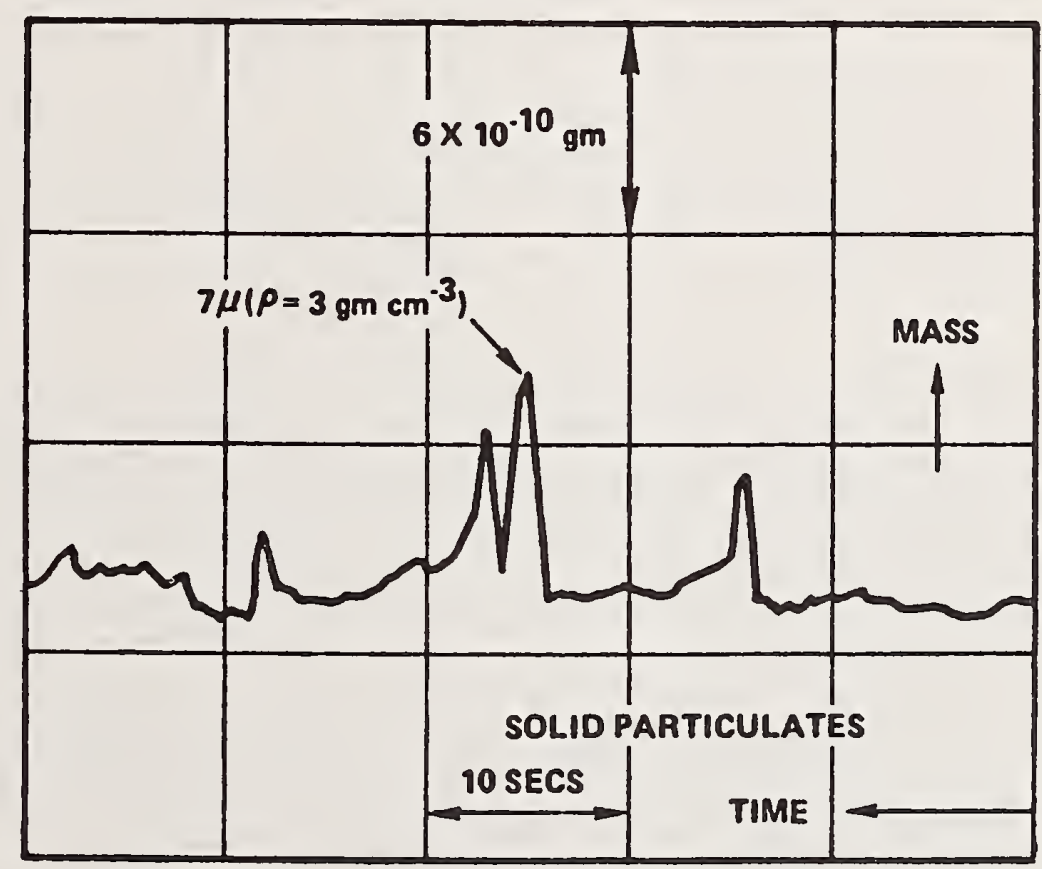

Figure 2. Differential spectra for solid particulates.

If the particulate matter incident on the crystal is of volatile material, some of the mass initially captured may be lost due to evaporation, which is also accompanied by some cooling of the crystal surface. The resulting response in the differentiated analog signal is a pulse followed by a negative pulse--the former due to the arrival of the mass, and the latter due in part to mass loss and in part to cooling. An example of this is shown in figure 3 . The pulses here are produced 


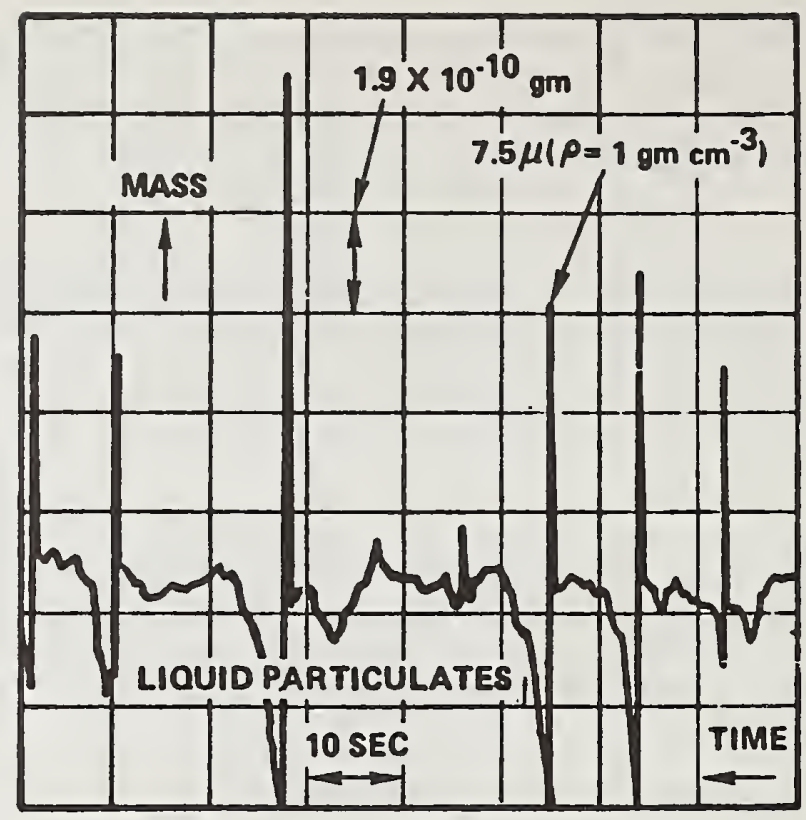

Figure 3. Differential spectra for volatile particulates.

by water droplets--the difference from the signals in figure 3 are quite apparent. The largest pulse here represents a water droplet of diameter 13 micrometers.

By analyzing a train of pulses it is possible to determine the distribution of mass with respect to size, as seen in figure 4 (from ref. 2). The particulate material used is a lapping grit made of a luminum oxide with mass density of $3.89 \mathrm{~g} / \mathrm{cm}^{3}$. (A mass of $10-10 \mathrm{~g}$ corresponds to a particle of $3.7 \mu$ diameter.) The distribution supplied by the manufacturer is shown for comparison with that determined by the QCM.

\section{PARTICULATE MASS CONCENTRATION MEASUREMENT}

With coincident arrivals of particles, some of which may also be too small to be resolved discretely, the total particulate mass concentration can be determined by measuring the cumulative mass change on the crystal with respect to time. The mass rate and the volume flow rate then yield the mass concentration. Figure 5 shows the temporal variation in the particulate mass concentration in a ventilated room when an aerosol hair-spray can is released for about 1 second, 2 meters from the instrument. In a smal1, unventilated room the concentration is seen in figure 6 to remain quite high for some time after the hair-spray application. 


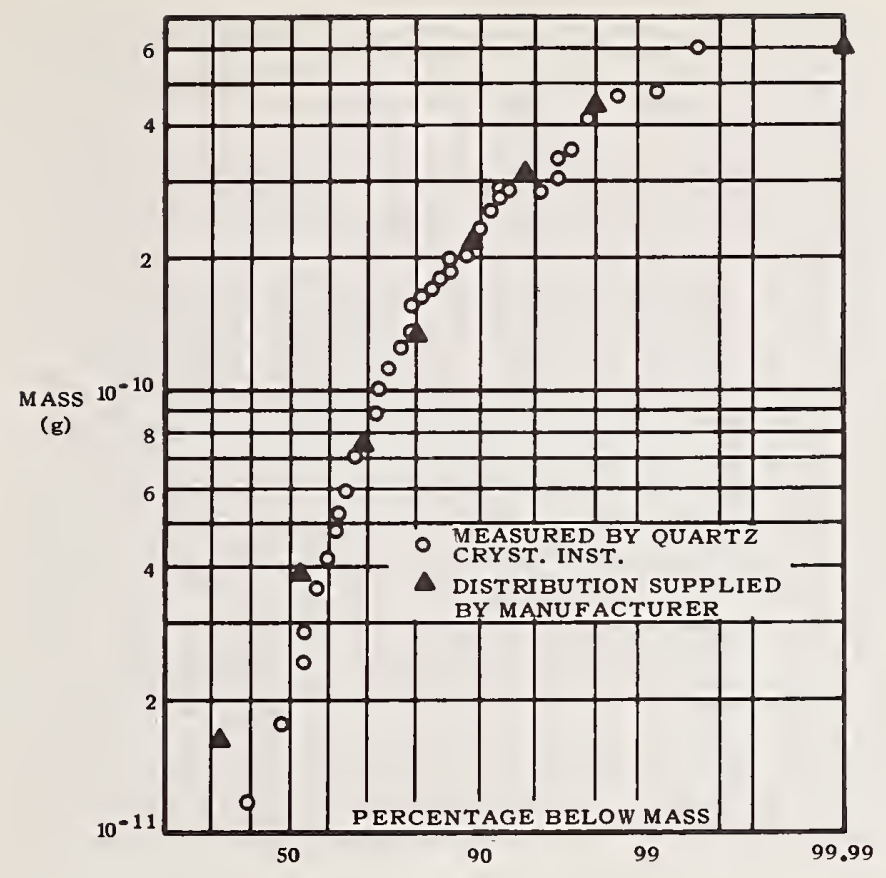

Figure 4. Particulate mass distribution curve obtained from a pulse height analysis of a train of pulses.

\section{POST SAMPLING ANALYSIS}

Since the adhesive-coated crystal retains the impacted material, post sampling analys is can be performed; and such analys is can be performed nondestructively and without disturbing the material by the use of scanning-electron-microscope (SEM), because the undisturbed crystal can be placed directly in a SEM. Figure 7 (from ref. 5) shows a $100 \mathrm{X}$ SEM of an entire impaction area (taken at normal incidence), measuring about 500 micrometers across, produced by exposing the crystal through a 400-micrometer impaction nozzle to smoggy atmosphere for about 10 minutes. The measured concentration was $250 \mu \mathrm{g} \mathrm{m}^{-3}$. At an impaction flow rate of $150 \mathrm{ml}$ min-1, this means a total mass accumulation on the crystal of about $0.38 \mathrm{\mu g}$. The symmetrical distribution of material, with smaller particles spread to the outer regions, is quite apparent. Figure 8 is a $10,000 X$ SEM (at 10 degrees tilt) of a part of the impaction area shown in figure 7 .

Figure 9 is a 200X SEM of the material collected from the plume of a solid-fueled rocket; while figures 70 and 11 show enlargements of two of the particles marked in figure 9. Particle 3 appears to be one of several cubical shapes, presumably salt crystals; while particle 2, along with many similar ones around it, appears to be spherical combus tion product from the rocket motor. Particle 1 is quite complicated in shape, and does not suggest anything readily identifiable. Results of $x$-ray analysis of these particles and of a reference table salt crystal are shown in table 1. 

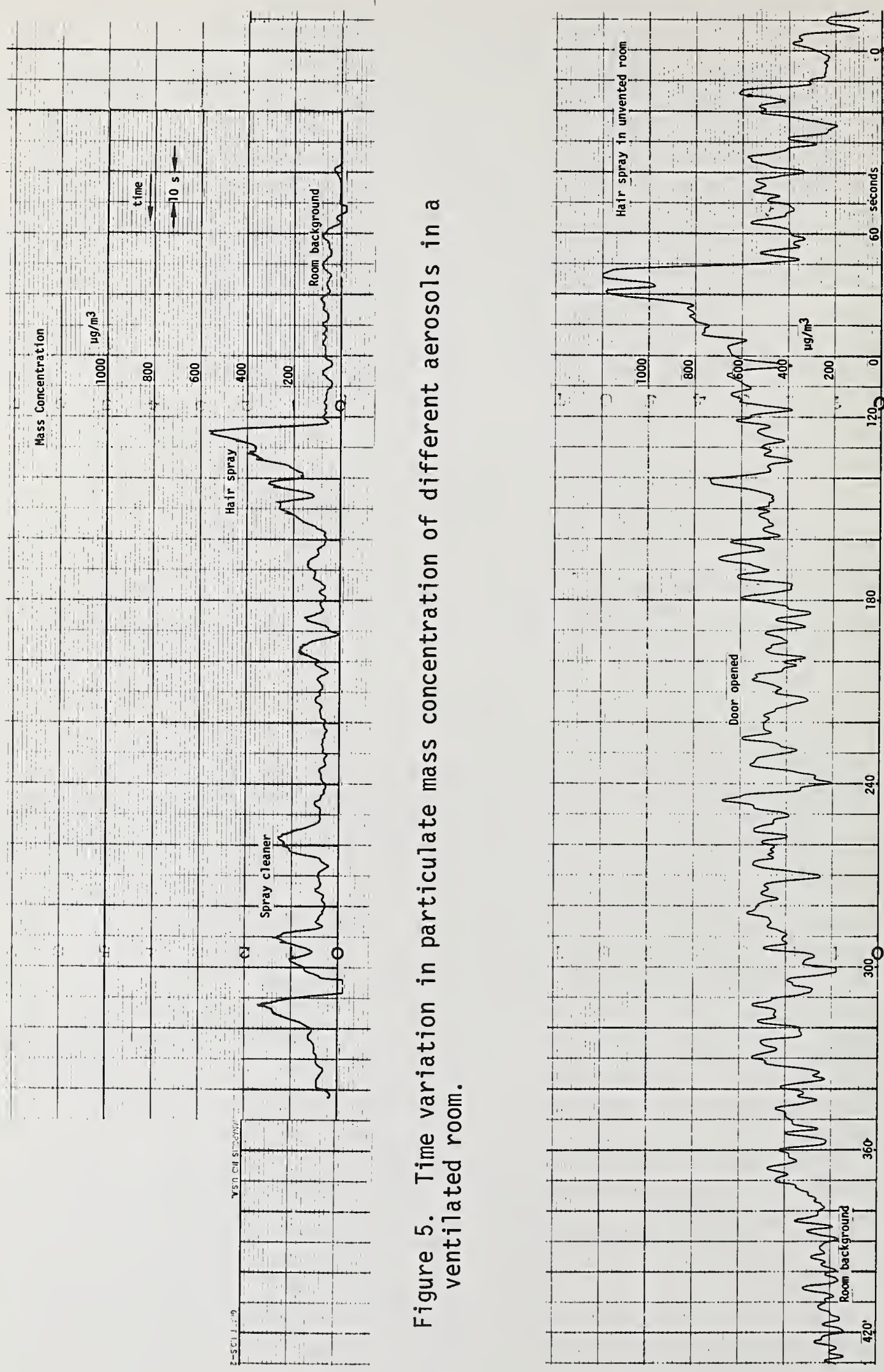

E் 


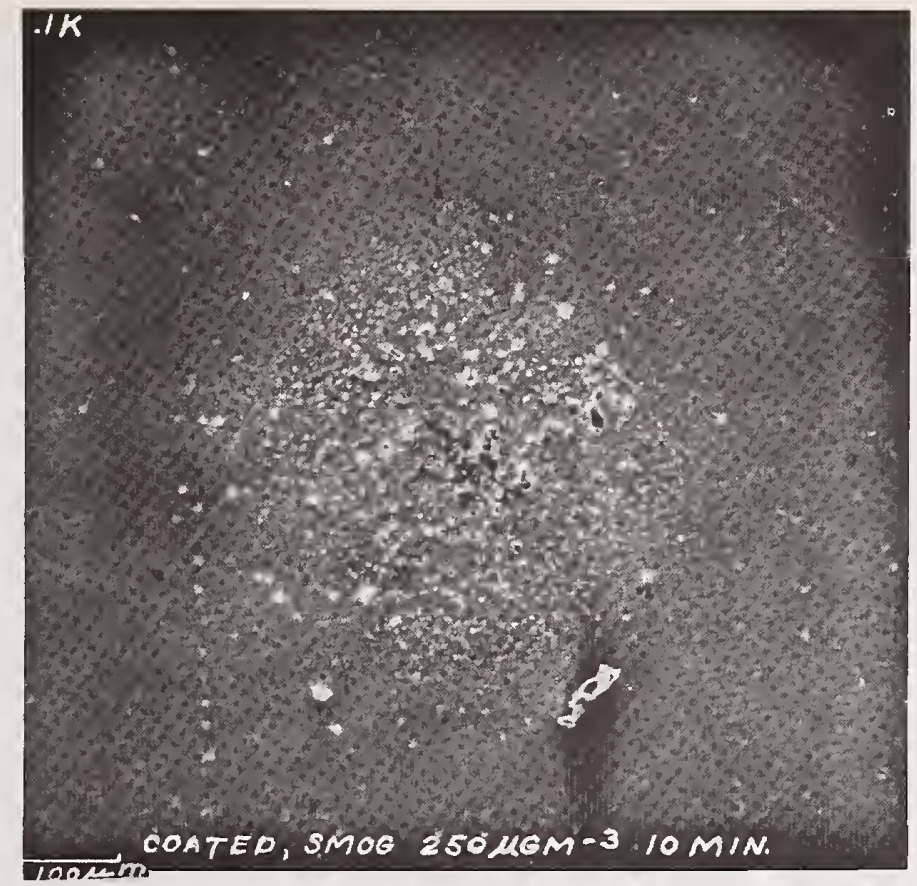

Figure 7. 100X SEM of impaction area of smog sample.

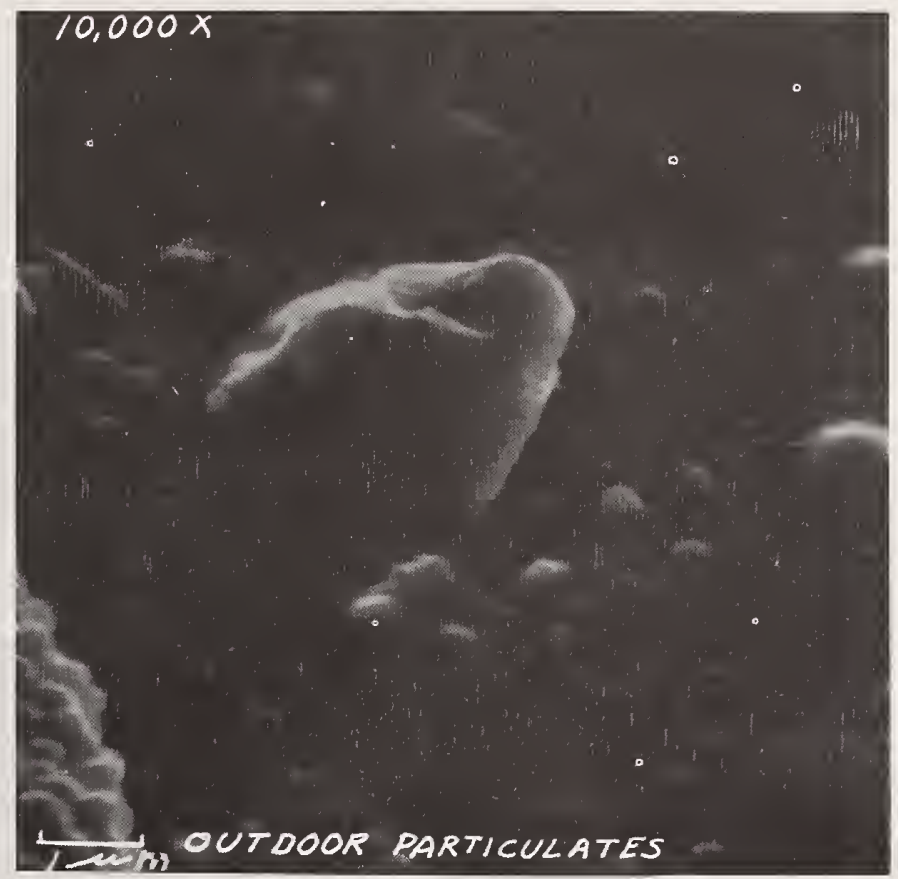

Figure 8. 10,000X SEM of impaction area of smog sample (same as in fig. 7). 


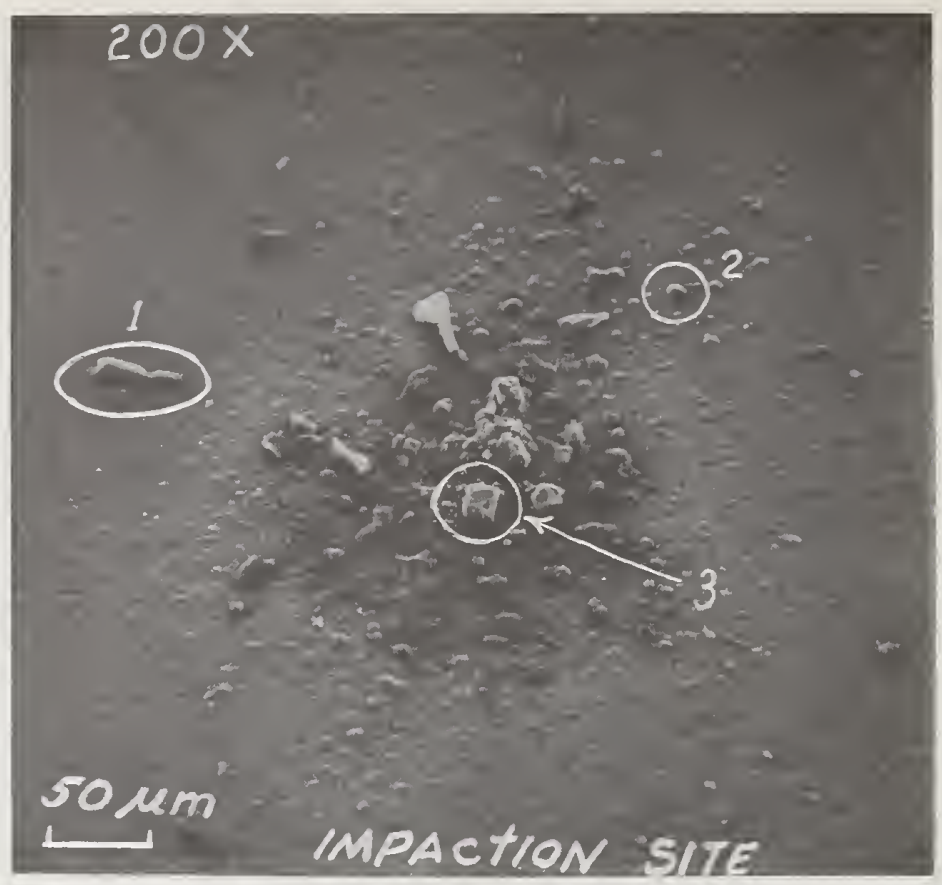

Figure 9. Impaction region from rocket plume $200 \times$ SEM.

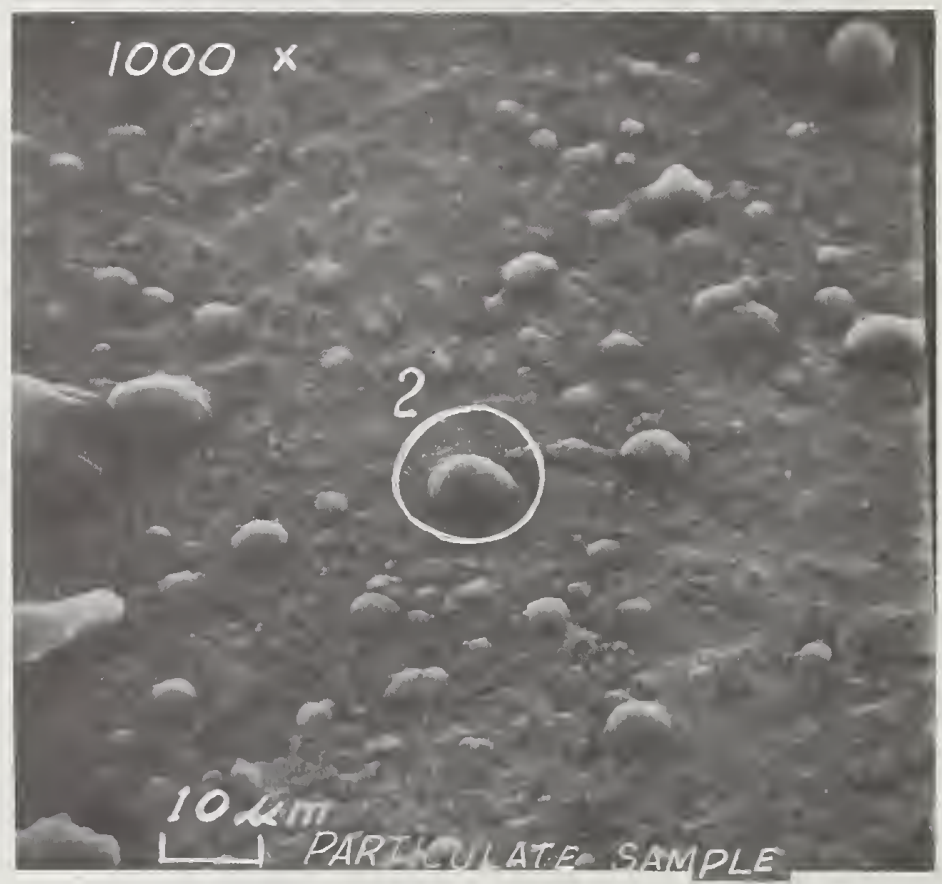

Figure 10. Impaction region from rocket plume $1,000 \times$ SEM. 


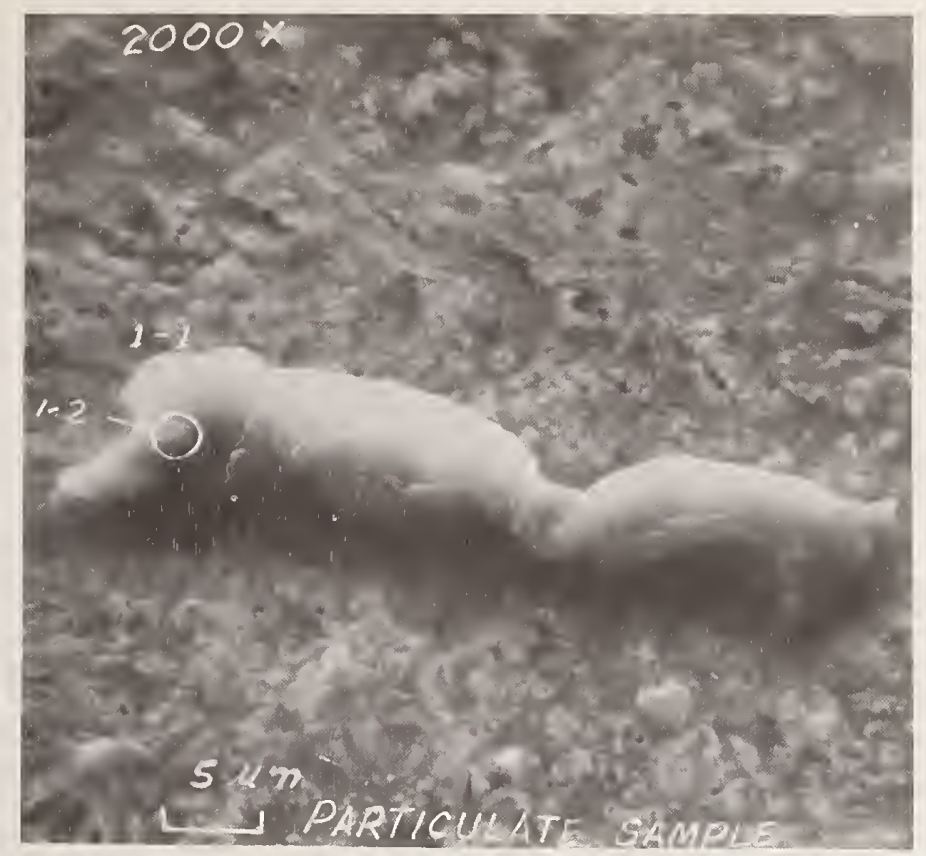

Figure 11. Impaction region from rocket plume $2,000 \times$ SEM.

Table 1. Molal fractions of particle samples.

\begin{tabular}{|c|c|c|c|c|c|}
\hline Element & $\begin{array}{c}\text { Particle } \\
1-1\end{array}$ & $1-2$ & 2 & 3 & Table Salt \\
\hline $\mathrm{Na}$ & 34.0 & 23.6 & & 50.3 & 41.0 \\
\hline Al & 9.1 & 14.8 & 70.7 & 1.2 & 11.4 \\
\hline Si & 11.0 & 18.0 & 2.9 & 0.8 & 6.5 \\
\hline $\begin{array}{l}\mathrm{An} \\
\mathrm{C} 1 \\
\mathrm{~K} \\
\mathrm{Ca} \\
\mathrm{Ti}\end{array}$ & $\begin{array}{r}38.3 \\
4.7 \\
2.8\end{array}$ & $\begin{array}{r}15.9 \\
5.6 \\
8.6 \\
6.8 \\
6.7\end{array}$ & 26.4 & 47.8 & 41.0 \\
\hline
\end{tabular}

It is seen that particle 2, with a preponderant percentage of aluminum, is probably a piece of aluminum oxide from the rocket motor. The presence of chlorine without, however, ariy accompanying sodium (as exhibited in other particles) suggests that the adsorption of hydrogen chloride (part of the rocket combustion product) by the aluminum oxide. From its shape and composition it is evident that particle 3 is salt-and a purer salt, in fact, than the reference table salt. Particle 1-1 is apparently made up of 68 percent salt and a few other materials. Particle 1-2, on the other hand, appears to be a different and much smaller particle adhering to the larger particle, and has a fairly complex makeup. 


\section{REFERENCES}

[1] Sauerbray, G. Z., Verwandung von Schwingquarzen zur Wägung Dunner Schlicht en und zur Mikrowägung, Z. Physik 155, 206-222 (1959).

[2] Chuan, R. L., An Instrument for the Direct Measurement of Particulate Mass, Journal of Aerosol Science, Vol. $\overline{1}$, No. 2, 111-114 (May 1970).

[3] Chuan, R. L., Particulate Contaminant Measurement by Quartz Crystal Microbalance, ASTM/IES/AIAA Space Simulation Conference, Sept. 1970.

[4] Chuan, R. L., Measurement of Particulate Pollutants in the Atmosphere, AIAA Journal, Vol. 10, No. 2, 1701-1704 (Dec. 1972).

[5] Chuan, R. L., Application of an Oscillating Quartz Crystal to Measure the Mass of Suspended Particulate Matter, 165th National Meeting of the American Chemical Society, Apr. 1973.

\section{DISCUSSION}

GEORGE SINNOTT: Is the negative overshoot a temperature effect?

CHUAN: It is a combination of negative mass rate and cooling. The concentration trace is a rate of mass of the crystal, so with the evaporation going on you actually get a negative. At the same time the cooling has the same effect.

ILAN CHABAY: Is there any effect of build-up of matter on the crystal surface?

CHUAN: Very definitely. You have to move your impactor site after a certain amount of accumulation because we have found empirically that is, for say particles of about one micrometer and down, when about 20 percent of the area that is available impaction area--about 20 percent of that area is occupied then the probability of a late arriving particle hitting on top of an already occupied site increases so that your response becomes nonlinear after that. Of course, with larger particles, you can put on more before that happens. So empirically this can be established, and for most of these things it's used mainly for diagnostic purposes of quick sampling where you can get information very quickly and then in between runs you can move your impaction site which can be done by rotating the nozzle or changing the crystal--a number of ways one can do that, and then be ready for the next run.

GEOFFREY HOTHAM: You mentioned that the device can measure spatially the hair spray, etc. Aren't you just measuring the impacted particles? 
CHUAN: When I say spatially, I only mean that in the sense you can move a cloud over the instrument or you can move the instrument through a cloud, and look at the spatial distribution of the concentration. I ' $m$ not talking about size distribution at any given point in time or space. I didn't make that clear.

AL GOLDING: But your probability of coincidence impaction would be pretty high and that would slow up your particle sizing when you are running with, say, an aerosol can.

CHUAN: I don't think it would be practical at all to use one device like this in the discrete mode to try to do pulse height analys is. It would be all mixed up. One obvious extension of this would be to cascade the device. We are in fact in the process of putting together one ten-stage and one six-stage cascade where each stage records mass continuously so that you can get a continuous distribution. It will work out to about a total mass concentration if you had a total mass concentration of the order of 100 micrograms per cubic meter then a reasonable central time to give you a good ten-step distribution would be about two minutes. Higher concentrations then you can do almost continuously.

EUGENE SCHEIDE: How does this instrument compare to the one built by Thermal Systems that uses an electrostatic precipitator?

CHUAN: Well, they are basically the same thing. The reason we went the impactor route is because as an aerodynamisist that's the only thing I can understand, and is tractable. any idea?

SCHEIDE: How does the collection efficiency compare? Do you have

CHUAN: We have run a number of comparisons and calibrations. For instance, we have done just direct optical comparisons. That is taking the crystal out and photomicrographically examined it. We periodically run it in parallel with the $\mathrm{Hi}-\mathrm{Vol}$ for instance. It is one of the reference methods and a comparison is usually quite good.

SCHEIDE: Do you achieve 100 percent collection?

CHUAN: That depends, of course, entirely on your impaction geometry. With the instrument which produces data that you see here it has a 50 percent cutoff at 0.2 microns. In the cascade the theoretical lowest cutoff is 0.05 micrometers at 50 percent. This is for mass density, not unit density.

MADHAV RANADE: These 0.05 micrometers, do they have to be operated at reduced pressure? stage.

CHUAN: Yes, it's about a quarter of an atmosphere in the tenth 
VIRGIL MARPLE: Have you done any study on particle bounce? Problems you have?

CHUAN: Yes, that is why we have an adhesive on it to make sure we don't have bounce. bounce?

MARPLE: Do you check the adhesive to make sure you don't have

CHUAN: Yes. We find that for particles, say generally--this is very rough--5 micrometers are larger and there is a tendency to bounce. Not only that, but beyond about 5 or 10 micrometers, they actually are not mechanically bonded to the surface. They sit there and rattle. You put the instrument on a very short time count in the high sensitivity and if you run it without an adhesive and put some large particles on, all you see is noise. You put the adhesive on it and you see it stays there and shows the increase in mass. 
NATIONAL BUREAU OF STANDARDS SPECIAL PUBLICATION 412

Aerosal Measurements, Praceedings af a Seminar on Aerasal Measurements

Held at NBS, Gaithersburg, MD, May 7, 1974 (Issued Octaber 1974).

DEVELOPMENT, CALIBRATION AND APPLICATION OF SIZE DISTRIBUTION INSTRUMENTS AT THE UNIVERSITY OF MINNESOTA

\author{
V. A. Marple \\ Mechanical Engineering Department \\ University of Minnesota \\ Minneapolis, MN 55455
}

\title{
ABSTRACT
}

Work with size distribution instruments has been in the areas of development, calibration, and application. Instruments have been developed in the area of particle size determination, such as the electrical aerosol analyzer, and in the area of monodisperse particle generation, such as the vibrating orifice generator and the differential mobility analyzer. These aerosol generators, along with new techniques, have been used to calibrate a variety of aerosol measuring instruments such as optical particle counters, nuclei counters, and the electrical aerosol analyzer. These instruments have then been used to study a range of particle types, from fairly ideal particles such as atmospheric aerosols to very nonideal particles such as coal dust.

Key words: aerosol electrical analyzer; aerosol generators; aerosol instrument calibration; aerosol size measurements; aerosol spectrometer; aerosol sprays; nuclei counters; particle generators; particle size measurements; particle size measurements by electromobility; particle standards.

\section{INTRODUCTION}

In the past few years the Particle Technology Laboratory of the Mechanical Engineering Department at the University of Minnesota has put considerable effort into the study of aerosol analyzing instruments. This work has been primarily in the areas of instrument development, calibration of aerosol sizing instruments, and the application of these instruments to field studies of real aerosols.

In the area of instrument development, four instruments will be discussed here. These are a size distribution determining instrument, the electrical aerosol analyzer; two aerosol generators, the vibrating orifice monodisperse aerosol generator and the submicrometer aerosol generator; and an eight-channel multichannel analyzer.

In the area of instrument calibration, the newly developed aerosol generators have been used to calibrate aerosol analyzing instruments such as the condensation nuclei counter, the electrical aerosol 
analyzer, and the optical particle counter with ease and accuracy previously not possible. Also, techniques have been developed to calibrate optical particle counters on nonideal aerosols such as coal dust particles.

By using these instruments and calibrations, several particle size analyzing systems have been developed and used in field studies. These systems include various numbers of sensors and data acquisition and analyzing capabilities. Three of these systems will be described and some results presented.

In the following sections, the above items are discussed more fully. For further details the reader is referred to the respective references.

\section{DEVELOPMENT}

Electrical Aerosol Analyzer (EAA)

(edited from Whitby and Liu, 1973)

The electrical aerosol analyzer is a size distribution measuring instrument with in-situ measurement capabilities over the 0.003 to $1 \mu \mathrm{m}$ diameter range. The operating principle of the device is that of electrical charging and mobility analysis, a principle first described by Whitby and Clark (1966). Recent advances (Liu, Whitby and Pui, 1973) in charger and mobility design and the use of all solid-state electronics have resulted in an improved instrument that is portable (about $30 \mathrm{~kg}$ in weight) and considerably more versatile.

Figure 1 is a schematic diagram of the instrument showing its major components: the aerosol charger, the mobility analyzer, the current sensor, and associated electronic and flow controls. The instrument samples aerosols at the rate of 5 liters per minute with an additional 45 liters per minute of clean air needed to operate the mobility analyzer.

In the instrument, the aerosol is first sampled into the charger where the particles are exposed to unipolar positive ions and become electrically charged. Figure 2 shows the relationship between the electrical mobility and the size of the particles under different charging conditions. It is observed that there is monotonic functional relationship between particle mobility and size. Using this relationship, the size distribution of the aerosol can be calculated from the mobility distribution measured by the mobility analyzer.

The mobility analyzer shown in figure 1 is in the form of a cylindrical condenser with clean air and aerosol flowing down the tube in a laminar stream. The charged aerosol particles are deflected through the clean air core by the voltage applied on the center electrode. For a given voltage on the center rod, particles above a certain critical mobility and size are precipitated, while those with lower mobility and a larger particle size escape precipitation and are 


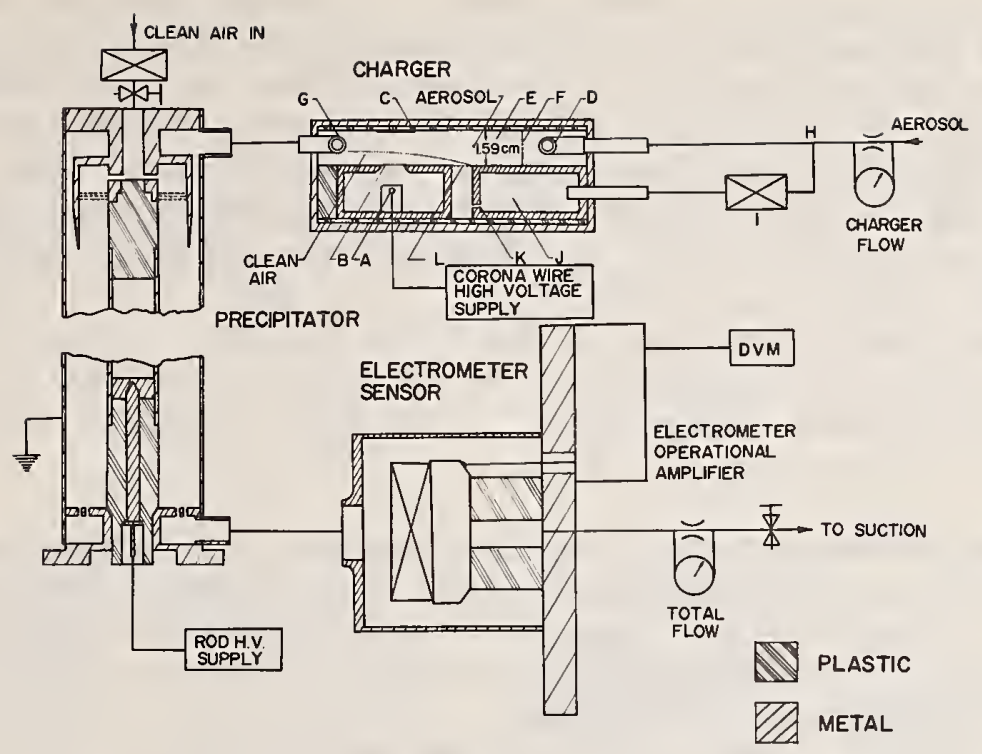

Figure 1. The portable electrical aerosol analyzer (Liu, Whitby and Pui, 1973).

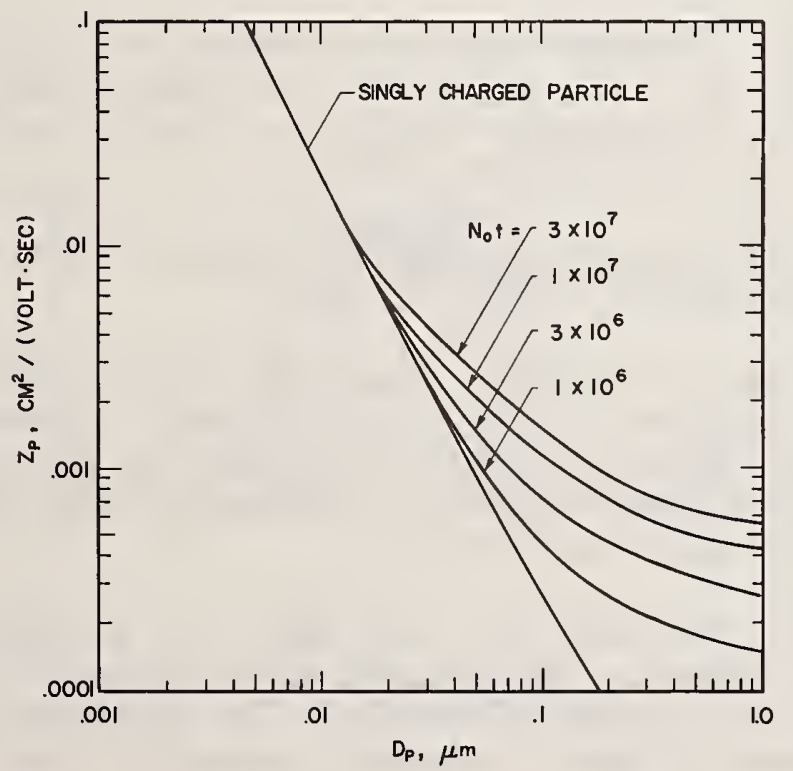

Figure 2. Relationship between electrical mobility and particle size for diffusion charging (Liu, Whitby and Pui, 1973). 
sensed by the electrometer current sensor. By changing the voltage on the center rod and measuring the corresponding electrometer current, the mobility and size distribution of the aerosol can be determined. The standard operating condition of the instrument provides a total of 11 voltage steps dividing the size range of the instrument into 10 equal geometrical intervals of four intervals per decade in particle size. The size interval boundaries are located at $0.0032,0.0056,0.01$, $0.0178,0.032,0.056,0.1,0.178,0.32,0.56$, and $1.0 \mu \mathrm{m}$. The complete voltage sequence can be scanned in about two minutes, thus allowing a complete size distribution analysis to be made in the same time period. The instrument has been interfaced to a mini-computer for complete automated data acquisition.

The standard deviation of number, surface, and volume measurement of the new instrument has been evaluated. The standard deviation of the electrometer current $\left(\sigma_{\Delta I}\right)$ ranges from a maximum of about 0.01 pa at $0.004 \mu \mathrm{m}$ to a low of about $0.001 \mathrm{pa}$ at $0.4 \mu \mathrm{m}$. This corresponds to standard deviations of $\pm 57,000 / \mathrm{cm}^{3}, \pm 0.4 \mu \mathrm{m}^{2} / \mathrm{cm}^{3}, \pm 0.003 \mu \mathrm{m}^{3} / \mathrm{cm}^{3}$ at $0.0042 \mu \mathrm{m}$ for number, surface, and volume respectively. At $0,75 \mu \mathrm{m}$ the corresponding standard deviations are $\pm 0.6 / \mathrm{cm}^{3}, \pm 1 \mu \mathrm{m}^{2} / \mathrm{cm}^{3}$, and \pm 1.1 $\mu \mathrm{m}^{3} / \mathrm{cm}^{3}$. It has been found that the variability is only significant for number distributions below $0.02 \mu \mathrm{m}$ and for the volume distribution above $0.5 \mu \mathrm{m}$. Therefore for most field work, data from the electrical analyzer has only been used for calculating sizes smaller than $0.422 \mu \mathrm{m}$. An optical particle counter has been used to measure the distributions for larger sizes.

The instrument has been used in a variety of laboratory and field studies and has proven its usefulness in these applications. The instrument is now available commercially from Thermo-Systems, Inc. (2500 Cleveland Ave. No., St. Paul, Minn. 55113).

Vibrating Orifice Monodisperse Aerosol Generator (VOMAG) (edited from Whitby and Liu, 1973)

The calibration of aerosol measuring and sampling devices is facilitated by the use of monodisperse aerosol. When the size or concentration of the aerosol (or both, depending on the application) are known to a sufficiently high degree of accuracy, the aerosol may be referred to as an aerosol standard.

During the past few years our laboratory has devoted much effort to the development of apparatus and procedures for generating monodisperse aerosols of a primary standard quality. Two approaches have been used for generating these primary standard aerosols, one involving the controlled disintegration of a liquid jet by a vibrating orifice, and the other the pneumatic atomization of a liquid and the electrostatic classification of the polydisperse aerosol to be described in the next section. The first method is applicable to larger particles over $0.5 \mu \mathrm{m}$ in diameter while the latter method is better suited for smaller particles below $0.5 \mu \mathrm{m}$. 
Figure 3 is a schematic diagram of the vibrating orifice monodisperse aerosol generator described by Berglund and Liu (1973). The generator consists of a droplet generation and dispersion system and an aerosol dilution and transport system. A Krypton-85 radioactive neutralizer of 10 millicurie activity is placed within the generator drying column to neutralize the particle electrostatic charge incurred during the droplet generation process.
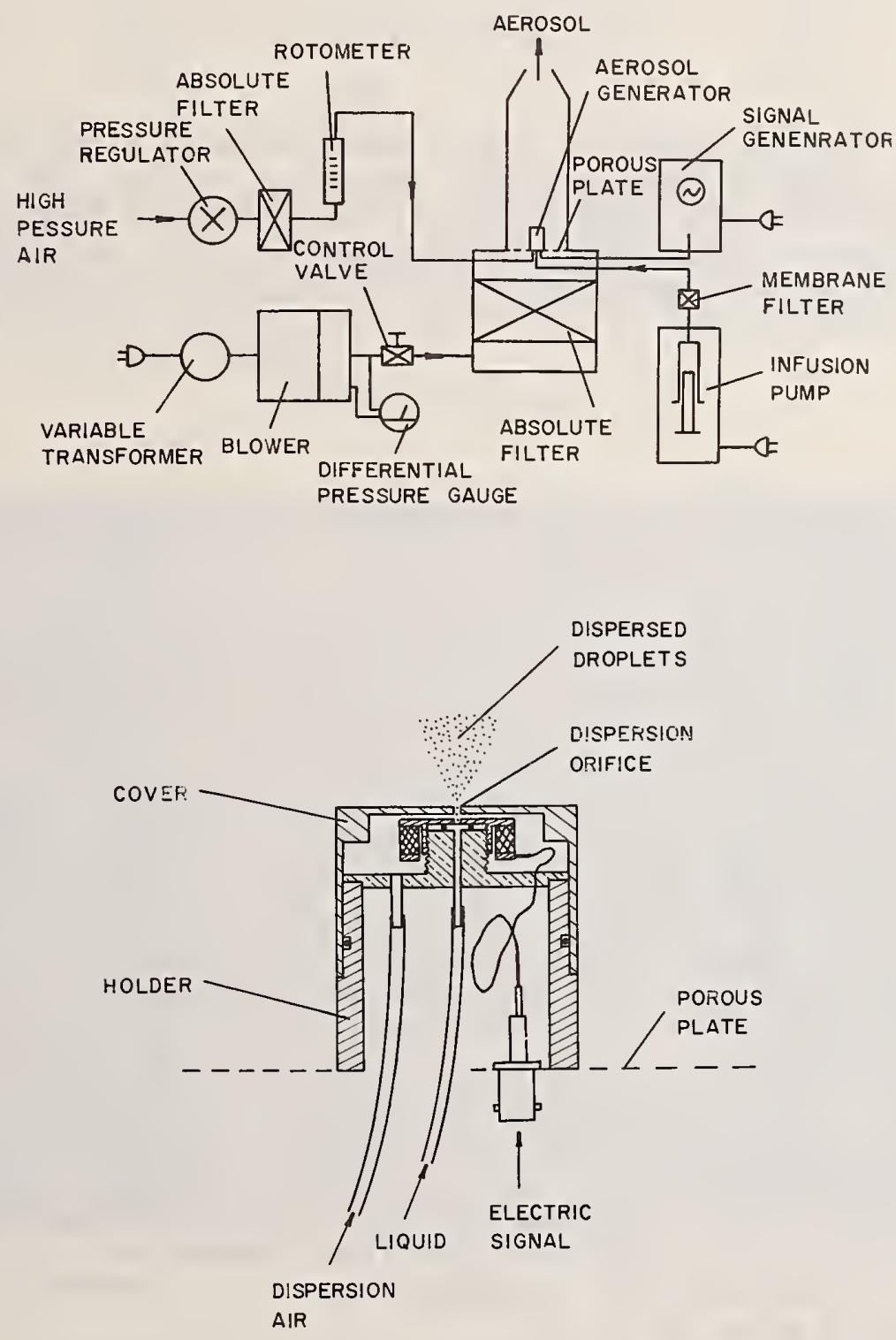

Figure 3. Vibrating orifice monodisperse aerosol generator (Berglund and Liu, 1973). Top: schematic of system. Bottom: generator head. 
In the vibrating orifice generator, uniform liquid droplets are generated by forcing a liquid through a small ( 5 to $10 \mu \mathrm{m}$ diameter), vibrating orifice by a syringe pump at a predetermined rate of $Q_{1} \mathrm{~cm} / \mathrm{s}$. The orifice is vibrated by a piezoelectric ceramic at a predetermined frequency of $\mathrm{f} \mathrm{Hz}$ supplied by a signal generator. Within an appropriate frequency range, the liquid jet is broken up into uniform droplets. since each cycle of the disturbance produces precisely one droplet, the individual droplet volume is equal to

$$
v_{d}=Q_{j} / f
$$

The uniform droplet stream is then injected axially along the center of a turbulent air jet to randomize the particle motion and to prevent particle collision and coalescence. The dispersed droplets are then mixed with a much larger volume of filtered dry air to evaporate the solvent and to obtain a stable aerosol of a particle diameter

$$
D_{p}=\left(\begin{array}{llll}
6 & Q_{1} & C / \pi f
\end{array}\right)^{1 / 3}
$$

where $C$ is the volumetric concentration of the nonvolatile aerosol material in the liquid solution.

Equation (2) shows that the diameter of aerosol particles produced by the vibrating orifice principle can be calculated from the liquid flow rate, $Q_{1}$, the frequency, $f$, and the solution concentration, $C$. Since these quantities can be easily measured to a high degree of accuracy, the particle diameter also can be calculated to a high degree of accuracy by means of eq (2). However, when dilute solutions (small C) are used, the nonvolatile impurity in the solvent must be taken into account in the calculation of particle diameter. Experiments (Berglund and Liu, 1973) have shown that with proper precautions, the particle diameter, $D_{p}$, can be calculated by means of eq (2) to a considerably higher degree of accuracy than can be measured by the conventional microscope techniques.

Table 1 is a summary of the operating conditions of the generator for three orifice sizes, and figure 4 shows some typical particles produced by the generator.

Theoretically the vibrating orifice generator can also produce aerosols of a known particle concentration. The theoretical aerosol concentration is given by

$$
N_{\text {th }}=f / Q_{a}
$$


Table 1. Characteristics of the vibrating orifice monodisperse aerosol generator.

Diameter

of

liquid orifice

um

5

Nominal

Droplet
diameter
$\mu \mathrm{m}$
15
25
40

Particle frequency

$\mathrm{kHz}$

450

10

225

20

60

diameter

Nominal ${ }^{c}$

range

$\mu \mathrm{m}$

concentration

particle $/ \mathrm{cm}^{3}$

$0.6-15$

273

$1.0-25$

137

$1.8-40$

36

\footnotetext{
${ }^{\mathrm{a}}$ Continuously adjustable over an approximate $25 \%$ range by varing the frequency.

Obtainable by the solvent evaporation technique.

${ }^{c}$ Theoretical concentration based on the nominal aerosol output of 100 liters per minute.
}

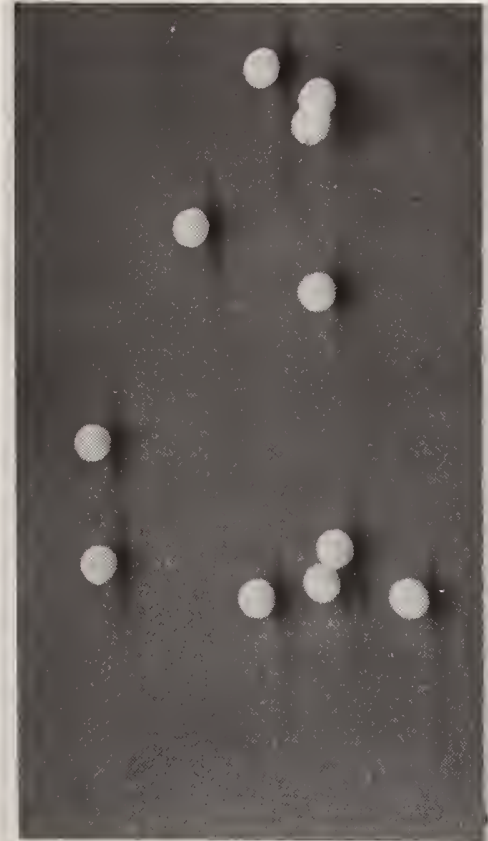

a

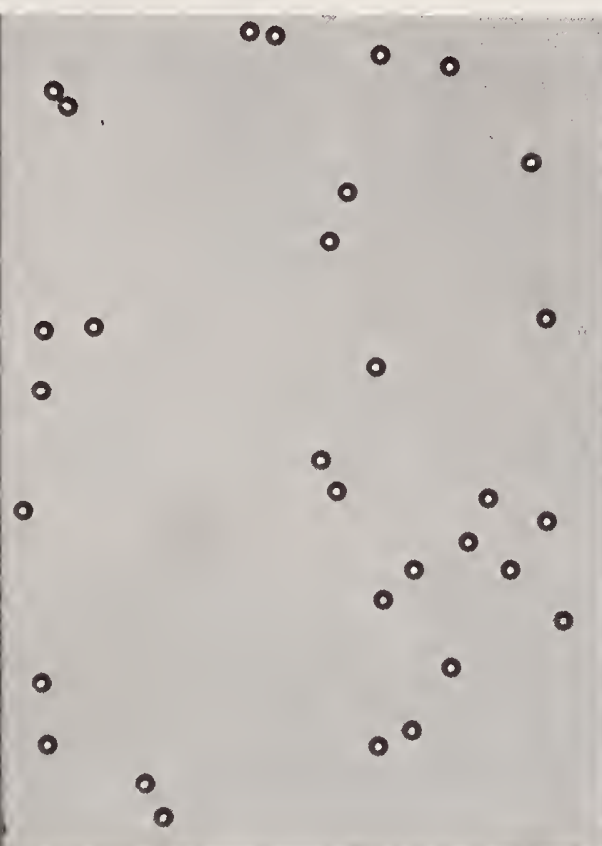

b

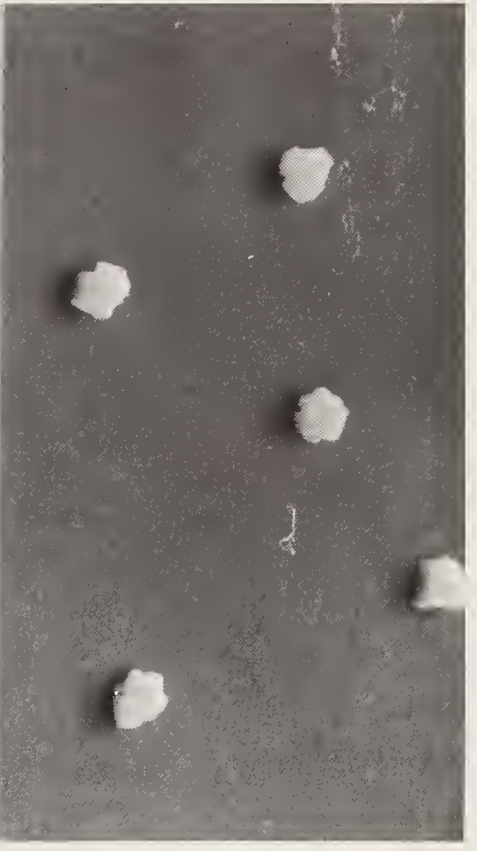

C

Figure 4. Monodisperse particles produced by the vibrating orifice generator (Berglune and Liu, 1973). a. $3.7 \mathrm{~m}$ diameter solid methylene blue particles. b. 9.5 micrometer diameter liquid DOP (di-octyl phthalate) particles collected on an oil-phobic slide c. solid sodium chloride particles of 27.4 cubic micrometer volume. 
The actual aerosol concentration at the generator output is less than the theoretical concentration due to particle loss in the drying and neutralization chambers and in the transport system. However, the operation of the generator is sufficiently stable so that after these losses are determined, the aerosol concentration at the generator output is known. Figure 5 (Liu, Berglund and Agarwal, 1973) shows the measured aerosol concentration at the generator output expressed as a percentage of the theoretical concentration and as a function of particle size. The actual concentration is seen to be about 81 percent $( \pm 5 \%)$ of the theoretical concentration for particles smaller than $6 \mu \mathrm{m}$ diameter, and the actual concentration decreases steadily with increasing particle size. This particular feature of the generator is very convenient for determining the sampling efficiencies of aerosol measuring and sampling devices. For instance, to determine the absolute sampling efficiency of an optical particle counter it is necessary only to apply the aerosol to the counter and to compare the counts calculated from the known aerosol concentration and the sampling flow rate of the counter. The generator has been used in a variety of studies including the calibration of optical particle counters (Liu, Berglund and Agarwal, 1973), impactor calibration studies, and turbulent deposition studies (Liu and Agarwa 1, 1973).

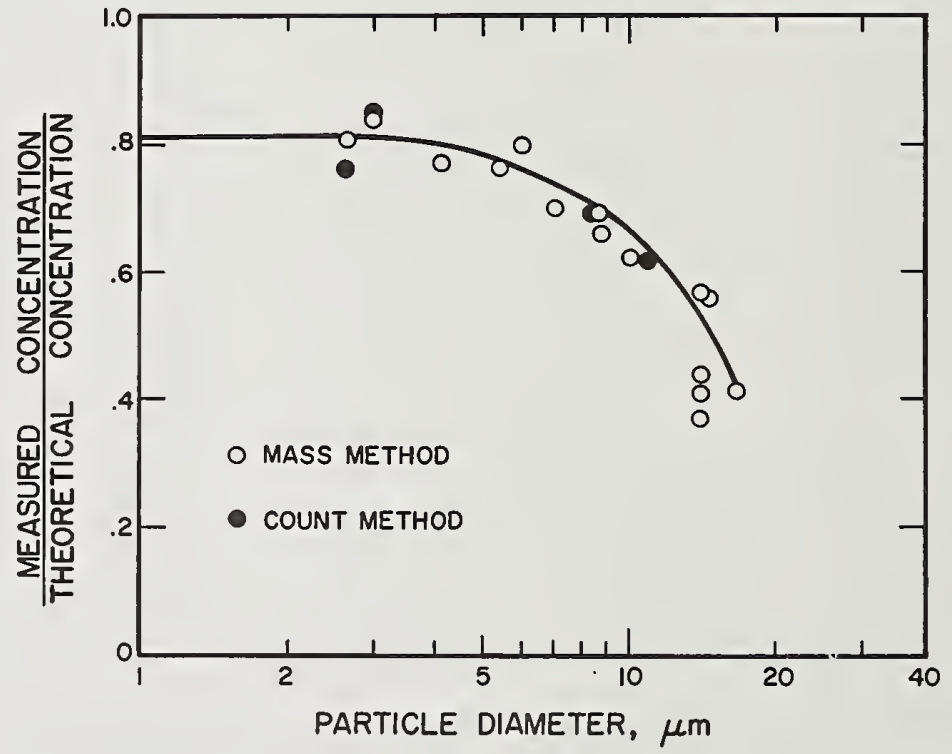

Figure 5. Output aerosol condensation of the vibrating orifice aerosol generator as a function of particle diameter (Liu, Berglund and Agarwa1, 1973). 
For generating submicrometer aerosol standards below $0.5 \mu \mathrm{m}$, the system shown in figure 6 is used. The device produces uniform particles at concentration levels up to $10^{6}$ particles $/ \mathrm{cm}^{3}$ by pneumatic atomization and electrostatic classification. The system consists of a Collison atomizer, a Krypton-85 radioactive neutralizer, a diffusion dryer, a differential mobility analyzer, and an electrometer current sensor.

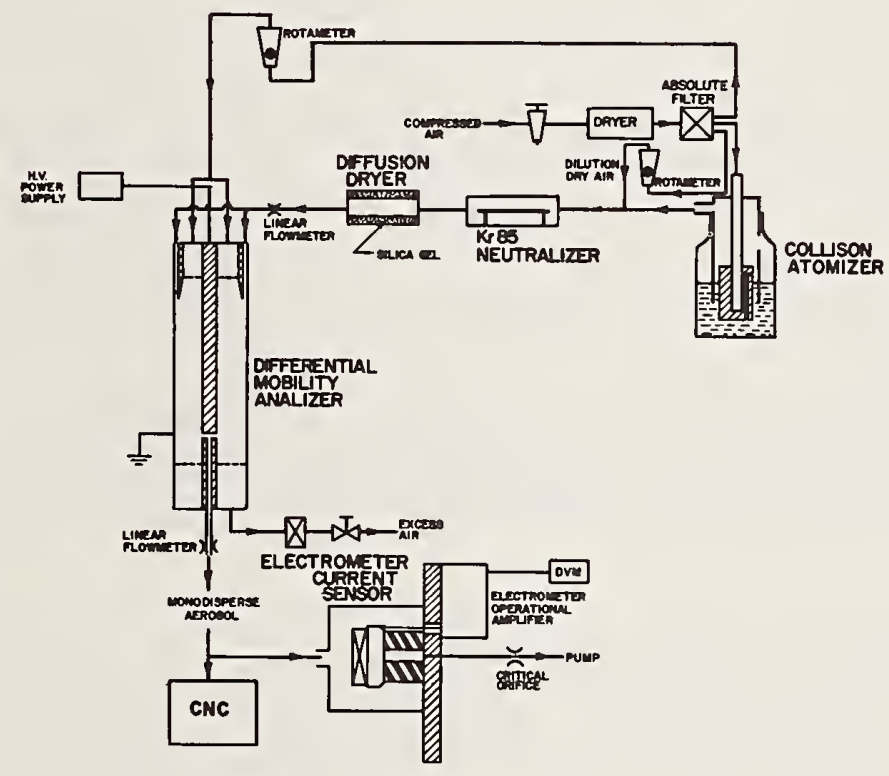

Figure 6. Apparatus for generating submicrometer aerosol standards (Liu and Pui, 1973).

In the apparatus a polydisperse aerosol is produced by the Collison atomizer. The aerosol particles are then brought to a state of charge equilibrium with bipolar ions produced by the ionizing beta radiation from the $\mathrm{Kr}-85$ source. Below a size of about $0.1 \mu \mathrm{m}$ diameter, most of the particles are either electrically neutral or carry one elementary unit of charge with an electrical mobility of

$$
Z_{p}=300 \mathrm{eC} / 3 \pi \mu D_{p}, \mathrm{~cm}^{2} / \mathrm{V}-\mathrm{s}
$$

where $e=4.8 \times 10^{-10}$ esu is the elementary unit of charge, $C$ is the dimensionless slip correction, $\mu$ is the gaseous viscosity, and $D_{p}(\mathrm{~cm})$ is the particle diameter. These singly charged particles can then be separated into monodisperse fractions by means of the differential mobility analyzer. 
The differential mobility analyzer shown in figure 6 is in the form of a cylindrical condenser with concentric electrodes. The inner electrode is held at a high voltage and the outer tube is grounded. Under a given set of operating conditions, charged particles in the aerosol stream flowing along the outer tube are deflected through the inner clean air core. If these particles have the appropriate electrical mobility, they would arrive at the exit slit near the lower end of the center electrode and be swept out by the air stream flowing through the slit. The electrical mobility of the particles is given by

$$
Z_{p}=\left[Q_{0}+1 / 2\left(Q_{s}-Q_{a}\right)\right] \ln \left(r_{2} / r_{1}\right) / 2 \pi V L
$$

where $Q_{0}\left(\mathrm{~cm}^{3} / \mathrm{s}\right)$ is the total flow in the mobility analyzer, $Q_{a}\left(\mathrm{~cm}^{3} / \mathrm{s}\right.$ is the aerosol flow at the inlet, $Q_{s}\left(\mathrm{~cm}^{3} / \mathrm{s}\right)$ is the sample aerosol flow through the slit and at the generator output, $r_{2}$ and $r_{1}(\mathrm{~cm})$ are the outer and inner electrode radii, $V$ (volts) is the applied voltage on the center rod, and $L(\mathrm{~cm})$ is the distance between the aerosol inlet and the exit slit.

The monodispersity of the aerosol at the generator output is determined by the ratio of flow $\left(Q_{5}+Q_{a}\right) / Q_{0}$. In the experiments reported by Liu and Pui (1973), the relative standard deviation of the aerosol ranges from 0.04 to 0.08 .

Since nearly all the particles classified by the differential mobility analyzer were singly charged and the percentage of multiply charged particles in the aerosol stream is sma11, particularly for small particle sizes, the absolute concentration of the aerosol can be measured by measuring the total current associated with the aerosol particles,

$$
I=q_{e} e^{N}
$$

The electrometer current sensor shown in figure 6 is used to measure this current, from which the aerosol concentration, $N$, can be calculated by means of eq $(6)$.

Eight-Channel Multichannel Analyzer

Commercial multichannel analyzers (MCA's) are designed to provide a high resolution of the signals to be analyzed. Thus most MCA's have the capability of at least 100 channels. However, in our laboratory, MCA's have been used to analyze the signals from optical particle counters, which, due to the limited size range of any one OPC (approximately 30 to 1 in particle size) and the desired resolution of the data, the data from the MCA is sorted into just a few groups. Thus a new MCA is being developed which has eight channels, making it unnecessary to group data from a large number of channels. Also, this MCA has analog outputs compatible with small analog data loggers. 
Not only does this simplified MCA aid in data reduction, but it is also relatively compact. One such unit is currently being equipped to operate on batteries so that it can be used with a portable OPC (Royco 218) to make a portable OPC-MCA system.

\section{CALIBRATION}

Calibration of Condensation Nuclei Counters (CNC) (by B. Y. H. Liu; edited from Whitby, T974)

The condensation nuclei counter is an aerosol concentration measuring device with useful applications in aerosol, air pollution, and meteorological research and in other scientific and technical work. The instrument is capable of detecting very small particles (to about $20 \AA$ in diameter) over a wide concentration range (from about 100 particles $/ \mathrm{cm}^{3}$ to $10^{7}$ particles $/ \mathrm{cm}^{3}$ ).

The Nolan-Pollak counter has been used for the past several decades as a calibration standard for other nuclei counters. Recently we have used the new submicrometer aerosol generator to perform a primary calibration on the Nolan-Pollak counter (Liu, et al., 1974). The results shown in figure 7 show that the Pollak calibration is in remarkably good agreement with the new technique.

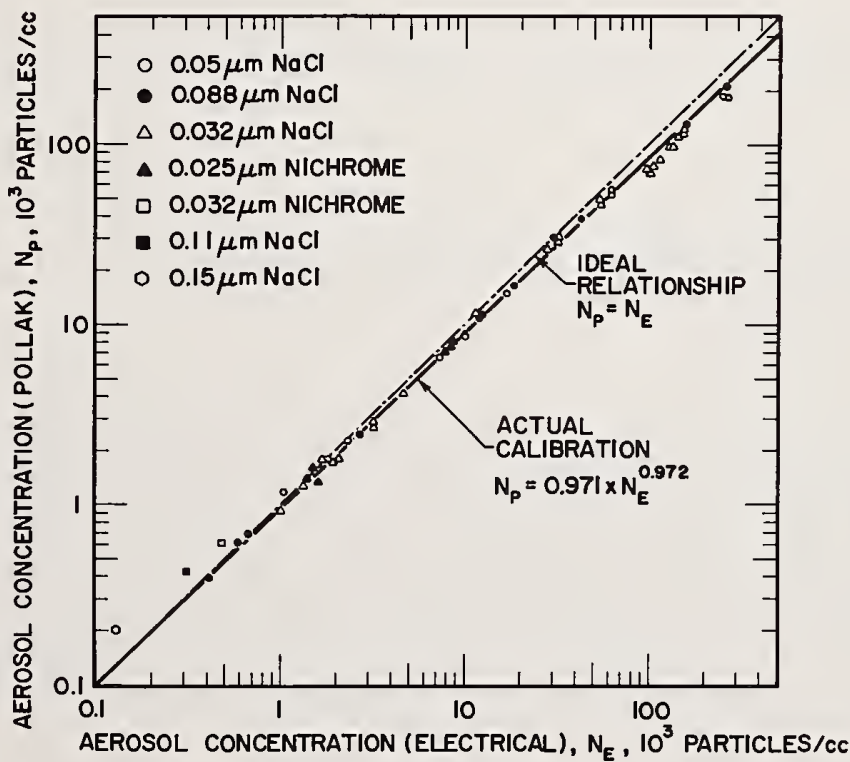

Figure 7. Calibration of the Nolan-Pollak condensation nuclei counter against the electrical aerosol standard. 
We have also used the new technique to calibrate automatic nuclei counters such as the Environment/One and General Electric instruments (Liu and Pui, 1973). A typical result for a factory-calibrated Environment/One counter is shown in figure 8 . It is seen from this figure that the indicated count is nonlinear and is low by a factor of 2.5 up to about 100,000 particles $/ \mathrm{cm}^{3}$ and by a factor of 6 at concentrations of 500,000 particles $/ \mathrm{cm}^{3}$.

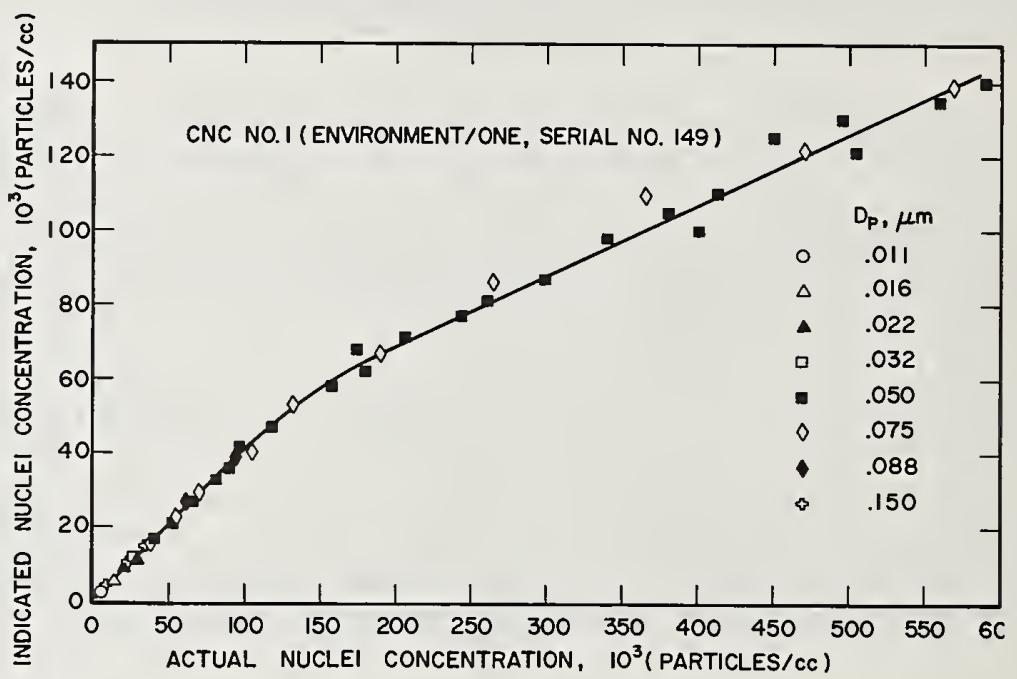

Figure 8. Response of the Environment/One condensation nuclei counter as a function of particle size and concentration.

Calibration of the Electrical Aerosol Analyzer

(by D.Y.H. Puí; edited from Whitby, 1974)

Because of the lack of a suitable aerosol standard, the calibration of the electrical analyzers was difficult. The calibration constants for the prototype unit were obtained using the earlier procedures of Husar and Whitby (Whitby et al., 1972). These procedures consisted of determining the space charge and diffusion loss experimentally. Further, some theoretical calculation of the instrument has to be included. This method does not take into account the spreading effect of the instrument. The detailed calibration of the EAA was thus limited due to a lack of a primary aerosol standard.

The development of the submicrometer aerosol generator (SAG) enables us to perform a detailed calibration on the EAA. The SAG can produce uniform particles of a known size and concentration over a wide range of values $(0.01 \mu \mathrm{m}$ to $0.5 \mu \mathrm{m}$ in diameter at concentrations up to $10^{6}$ particles $/ \mathrm{cm}^{3}$ ). The particle size can be calculated to an accuracy of 2 percent and the concentration to an accuracy of 5 percent. 
Therefore, it is particularly suitable for the primary calibration of the EAA. The calibration is currently underway and has yet to be completed. The outline of the calibration plan and the results of preliminary data are briefly described below.

It is required that the aerosol charge distribution be reduced to Boltzmann's equilibrium before being introduced into the EAA. Therefore, the singly charged particles from the SAG must first be passed through a $\mathrm{Kr}-85$ radioactive neutralizer in order that they may attain the condition of charge equilibrium. The concentration must then be determined by means of another instrument in place of the Faraday's cage. The instrument used here for measuring concentration is an Environment/One Condensation Nuclei Counter (CNC). Its calibration was previously standardized against the SAG. Particles passing through the neutralizer are first measured by the CNC before being introduced into the EAA. Since the aerosol flow for the EAA was $41 \mathrm{pm}, 11 \mathrm{pm}$ of clean air must be added to the $3 \mathrm{lpm}$ of aerosol output from the SAG.

$\mathrm{NaCl}$ aerosols with diameters at the geometric midpoint of the standard size intervals of the EAA, namely, .024, .042, .075 um, etc., were fed into the EAA. For each particle size a set of current vs. voltage was obtained. Table 2 lists the data that we have obtained thus far. The $.042 \mu \mathrm{m}$ diameter particles are generated by the vibrating orifice generator (Berglund and Liu, 1973). Due to the large diameter reduction (more than 20:1) for generating these small particles, the data may not be accurate.

Note that for particles larger than $.075 \mu \mathrm{m}$, the largest current drop occurs in the size interval larger than the nominal size interval of the EAA. This indicates that the presently used voltage for these larger particle size intervals is in error and needs to be adjusted later when the preliminary calibration is completed. The spread in current seems to decrease as the particle diameter decreases. For the $.042 \mu \mathrm{m}$ diameter, the current drop vs. size shows two peaks at $.042 \mu \mathrm{m}$ and $.133 \mu \mathrm{m}$ corresponding to singly and doubly charged particles of the chosen size.

The $\mathrm{NaCl}$ aerosol generated by the Collison atomizer has a geometric standard deviation of 2.65. It is therefore necessary to consider the interference effects of double charged particles from the SAG. For this reason we plan to continue the calibration of the EAA with the condensation aerosol generator (CAG) in place of the Collison atomizer in the SAG. With a geometric standard deviation of 1.3 for the CAG, the interference effects can be ignored. 
Table 2. Preliminary calibration data for the EAA.

\begin{tabular}{|c|c|c|c|c|c|}
\hline \multirow{3}{*}{$\begin{array}{l}E A A \\
D_{p i^{-\mu m}}\end{array}$} & \multicolumn{5}{|c|}{ I, $10^{-12}$ ampere. } \\
\hline & \multicolumn{5}{|c|}{ Mean particle size (generated by $S A G$ ) $-\mu m$} \\
\hline & .024 & .042 & .075 & .133 & .42 \\
\hline .0042 & 0 & 0 & 0 & 0 & 0 \\
\hline .0075 & 0 & 0 & 0 & 0 & 0 \\
\hline .0133 & .001 & 0 & 0 & 0 & 0 \\
\hline .0237 & .029 & 0 & 0 & 0 & 0 \\
\hline .0422 & .009 & .101 & 0 & 0 & 0 \\
\hline .0750 & 0 & .06 & $.20\} a$ & .02 & 0 \\
\hline .133 & 0 & .1051 & $.27\}^{a}$ & $.27\} a$ & 0 \\
\hline .237 & 0 & .002 & .042 & $.27\}^{a}$ & 0 \\
\hline .422 & 0 & .003 & .025 & .12 & $.014\}$ \\
\hline .750 & 0 & 0 & .016 & .026 & $.018\}$ \\
\hline
\end{tabular}

apreliminary calibration on the first production TSI Model 3030 EAA. The indicated particle sizes have been found to be larger than the true sizes. This is primarily due to the fact that the charger on this instrument was designed from theory and the precipitator voltages were not adjusted prior to calibration. A new voltage program chip is being designed to adjust the observed sizes to agree with the true sizes.

Calibration of Optical Particle Counters (OPC)

Considerable work has been done in our laboratory on calibrations with very monodisperse ideal particles (spherical oil droplets of known refractive index) generated on the vibrating orifice monodisperse aerosol generator. Work has also been done on calibration with nonideal particles such as coal or rock dusts.

If the ideal particles from the Vibrating Orifice Monodisperse Aerosol Generator (VOMAG) are used, the calibrations are relatively simple. Figure 9 illustrates the pulse height spectrum, as recorded by an MCA, of a counter exposed to aerosols produced by this method. In figure $9(a)$ the aerosol is essentially monodisperse as indicated by the 

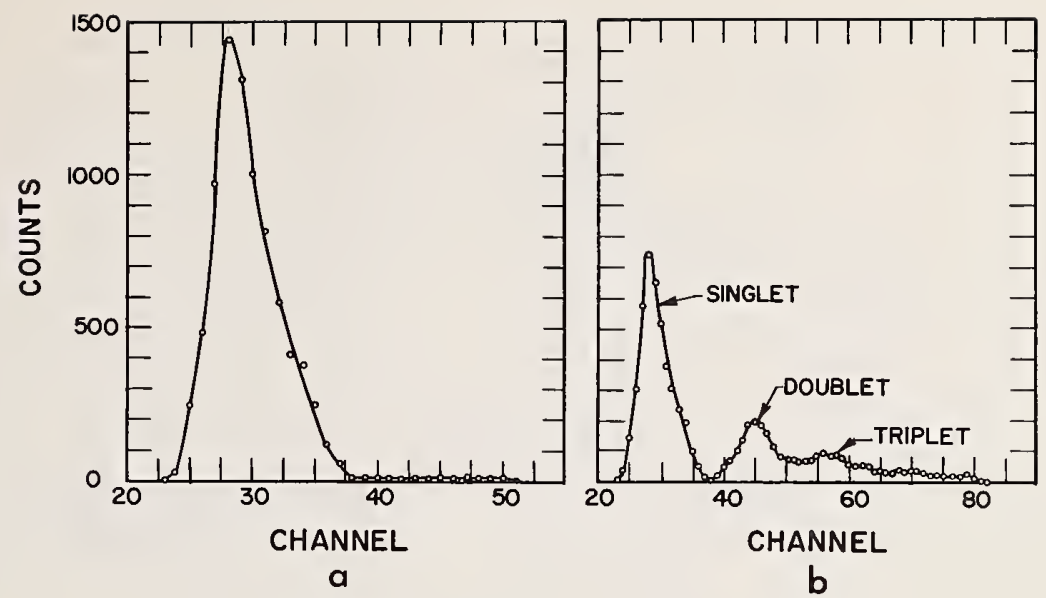

Figure 9. Pulse height spectra of an optical particle counter exposed to aerosols from the vibrating orifice aerosol generator (Liu, Berglund and Agarwal, 1973). a. under good dispersion conditions b. under poor dispersion conditions.

pronounced peak in the spectrum. In figure $9(b)$ additional peaks appear which are produced by particles with two, three, or more times the volume of the primary particle volume. These multiplet particles are obtained by deliberately operating the vibrating orifice generator under poor dispersion conditions to increase the collisional frequencies between the primary droplets. Figure 9(b) shows that for the specific counter studied, the singlets and doublets with a volume ratio of $1: 2$ or a diameter ratio of $1:(2) 1 / 3$ or $1: 1.15$ are also resolved by the counter. The triplets and quadruplets with diameter ratio of $1: 1.10$ are not clearly resolved.

Although calibration is relatively simple for ideal aerosols shown in figure 9, calibration with nonideal aerosols is considerably more difficult. Such a calibration has been made on a Bausch and Lomb OPC to size coal and rock dust particles.

The calibration was accomplished in two stages (Liu, Marple, Whitby and Barsic, 1974). For particles smaller than $2.4 \mu \mathrm{m}$, monodisperse coal dust was supplied to the OPC by using the differential mobility analyzer identical to that used in the submicrometer aerosol generator. For particles larger than $2.4 \mu \mathrm{m}$, a polydisperse coal aerosol was sized by the OPC and the resulting MCA output compared to the particles, sized by microscopy, which passed through the MCA.

In the first stage ( $\leq 2.4 \mu \mathrm{m}$ calibration), monodisperse aerosols of coal were generated by the system shown in figure 10. A small quantity of finely ground coal is first aerosolized by means of the fluidized-bed dust feeder and subsequently passed through the $\mathrm{Kr}-85$ aerosol charge neutralizer to reduce the particle electrostatic charge. The aerosol 


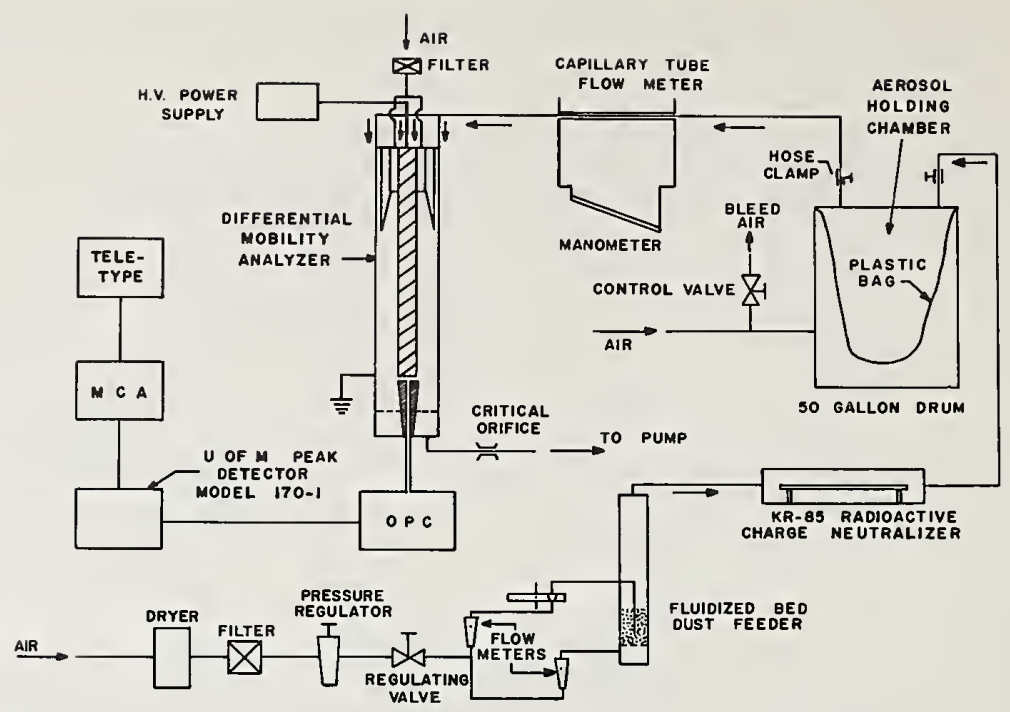

Figure 10. System for generating monodisperse coal aerosols for calibrating optical particle counters.

then enters a 0.2 cubic meter holding chamber where it is mixed with a much larger volume of clean filtered air. After a sufficient quantity of coal dust has been generated and an adequate dust level has built up in the chamber, the dust feeder is turned off. The aerosol from the chamber is then passed through the differential mobility analyzer to obtain particles within a narrow mobility and particle size range, which are the calibration particles for the OPC. The value of the signal at the mode of the distribution was used as the calibration for any specific size.

In the second state ( $<2.4 \mu \mathrm{m}$ calibration), a polydisperse coal aerosol within the appropriate size range was generated by means of the fluidized-bed dust feeder as before, and the particles were sampled directly into the OPC. The pulse height distribution was measured by the MCA; simultaneously, these particles were collected by a membrane filter after they had passed through the viewing volume. The collected particles were subsequently measured by an optical microscope fitted with a Portron reticle. The particle diameter was based on an equivalent circle having an area the same as the projected area of the particle. Further analysis of the MCA and microscope data gave the required calibration data in the large particle size range.

The resulting calibration from these techniques is given in figure 11. This shows the ideal Dioctyl Phthalate (DOP) calibration curve as well as the coal calibration. Note that for the larger sizes a coal particle must be about five times as large as an ideal particle to give the same signal level. 


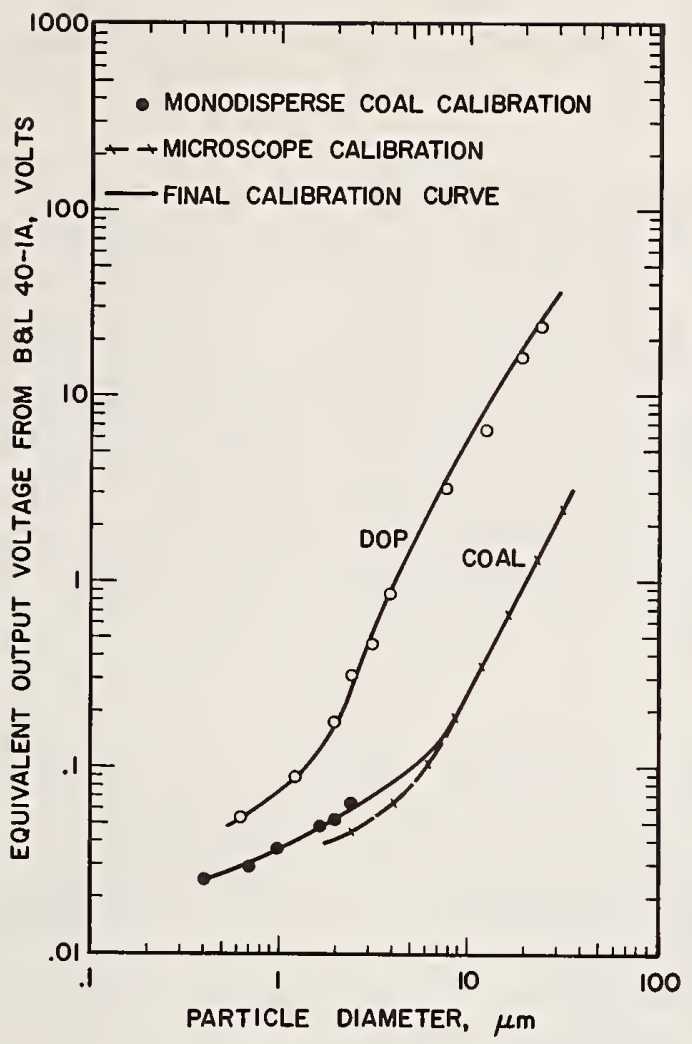

Figure 11. Calibration curve for the Bausch and Lomb 40-1 or 40-1A Dust Counter.

\section{APPLICATION}

Instrument Systems for Aerosol Size Distribution Measurement (edited from Whitby and Liu, T973)

Several aerosol size distribution measurement systems have been built in our laboratory. Two of these systems were made mobile by installing them in a semitrailer. One trailer was delivered to the Air Resources Board of the State of California and the other was delivered to the Environmental Protection Agency. Since both of these systems were used to study atmospheric aerosols, the instrumentation and trailer construction were similar.

Because of the broad range of sizes in most aerosols of interest, and because of steep decreases in particle number with increasing size, it is ordinarily not desirable to measure more than about one and onehalf decades of size with any one instrument. Therefore, for our field aerosol measurement work we have developed instrument systems which combine a nuclei counter, an electrical counter, and up to three optical 
counters in a single system. A mini-computer is used to control the counters and to calculate a single size distribution from the different instrument outputs.

Figure 12 is a schematic of a mobile air pollution laboratory which we designed, built, and operated for the Air Resources Board of the State of California in 1972 (Whitby et al., ]973). This used an Environment/One CNC, a Thermo-Systems, Inc. Model 3000 Whitby Aerosol Analyzer for the 0.0075 to $0.42 \mu \mathrm{m}$ range, and a modified Royco 245 for the 5.6 to $38 \mu \mathrm{m}$ range. It is interesting to note that total aerosol volumes measured with this system are within \pm 30 percent of the volumes calculated from total filter mass measurements. This indicates that with proper calibration and operation these automatic instruments can achieve good absolute accuracy as well as providing ease of operation and speed of measurement.

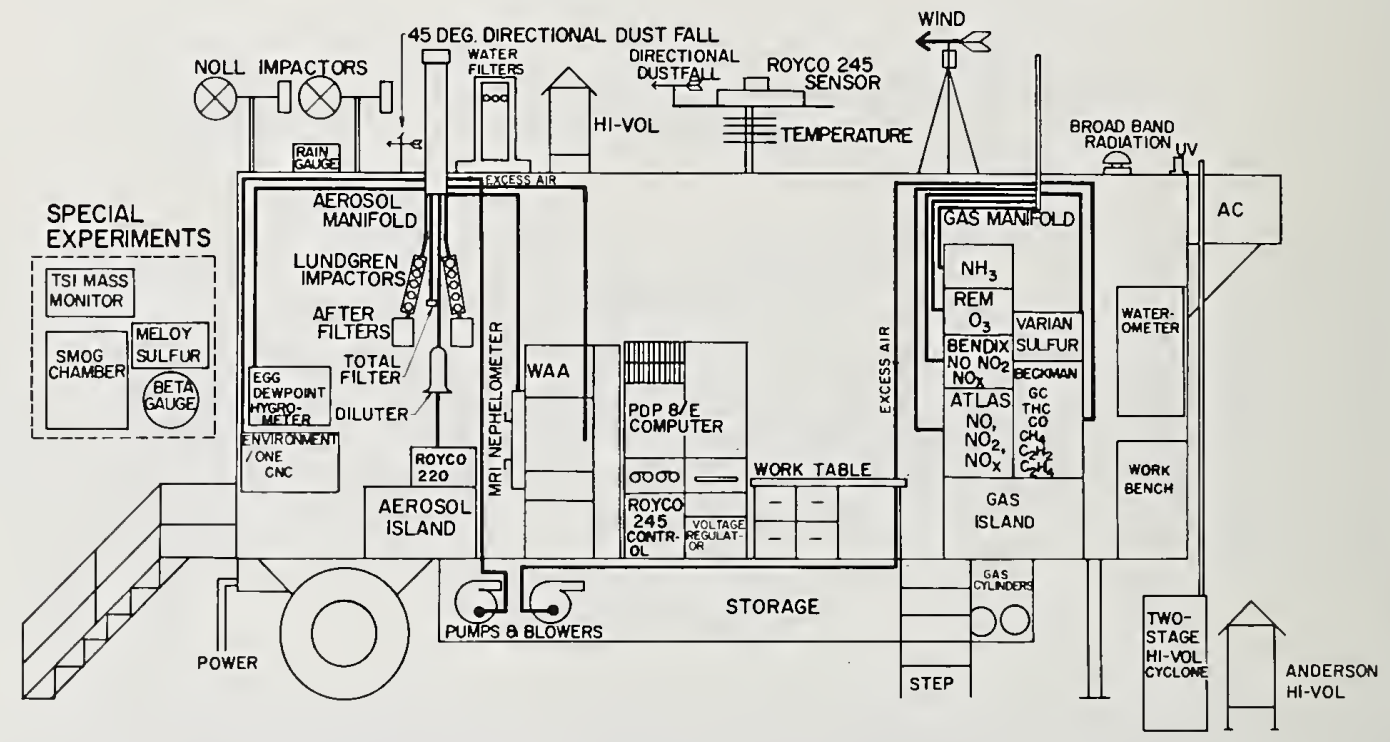

Figure 12. Schematic of a large mobile air pollution laboratory constructed for the Air Resources Board of the State of California. This laboratory includes a comprehensive array of automatic aerosol size analyzers, particle samplers, gas analyzer, and meterological instruments (Whitby et al., 1973).

Another system built in our laboratory was delivered to the Bureau of Mines for the study of coal dust distributions in coal fragmentation tests. This system was designed to measure the concentration and size distribution dust in the 0.005 to $20 \mu \mathrm{m}$ diameter range. As shown in figure 13, three sensors are used in the system together with a data acquisition system: the Whitby Aerosol Analyzer for size distribution 


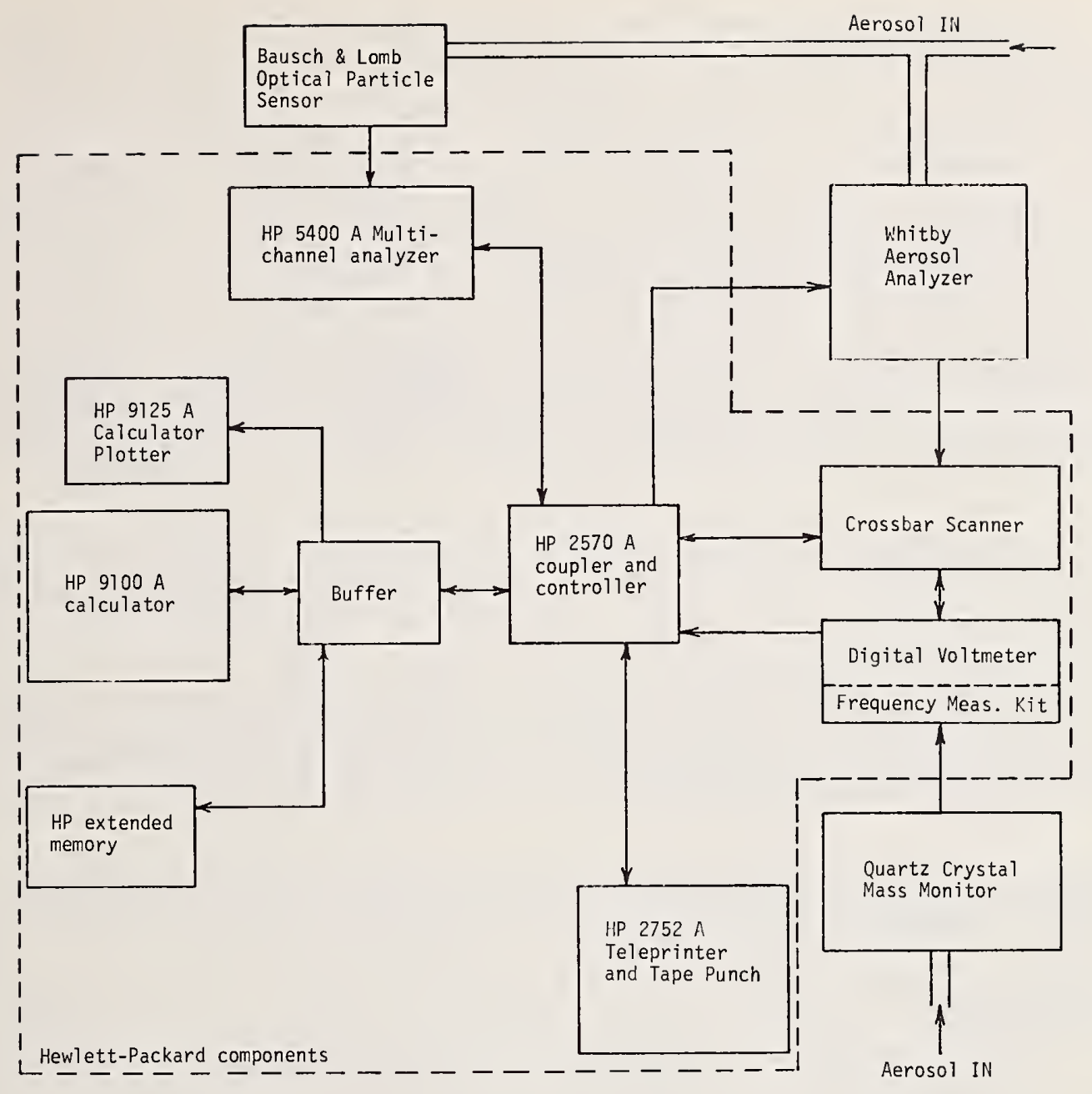

Figure 13. Block diagram of data acquisition and data reduction system for the coal dust measuring system.

measurement in the 0.005 to $0.5 \mu \mathrm{m}$ range, the Bausch and Lomb optical particle counter for the 0.5 to $20 \mu \mathrm{m}$ range, and a quartz crystal mass monitor sensor to measure the mass concentration of the coal dust.

The data acquisition system is comprised of several pieces of electronic equipment shown within the dashed area in figure 13. It is a Hewlett-Packard calculator-based system consisting of a multichannel analyzer, a digital voltmeter with frequency measuring capability, a calculator, a teleprinter, a plotter, and a crossbar scanner connected through a coupler/controller. The coupler/controller serves as the master control for the entire system, but can then issue commands to other pieces of equipment in the system. Since the mass concentration can also be calculated from the size distribution data, the value can be compared with the values determined directly by the quartz crystal mass monitor. 
When the data acquisition and reduction system is used to determine the size distribution of coal dusts, a "reset" or "start" button is depressed on the coupler/controller. The coupler/controller then instructs the WAA to begin operation and the multichannel analyzer to begin accumulation of counts from the OPC. The WAA then steps through its internal voltage sequence. At the same time, the MCA records signals from the OPC for a predetermined length of time. After stepping to a new voltage and after allowing about 15 seconds for the electrode current to settle to a steady value, the WAA sends a read signal to the coupler/controller, which in turn commands the digital voltmeter to take a current reading. This current reading is then stored in the calculator's extended memory. The calculator is then instructed to determine the size distribution from the WAA and the OPC data for printout on the teleprinter or graphical display on the plotter.

The crossbar scanner enables the voltmeter to read data from various sources, such as the WAA, strain gages, temperature sensors, humidity sensors, and other instruments whose signal needs to be recorded by the same central data acquisition system.

The digital voltmeter includes a frequency measuring option which enables the system to accept the frequency signals generated by the quartz crystal mass monitor.

Summary of Current Knowledge of Fine Particles in the Atmosphere (by K. T. Whitby, 1973)

Introduction

This brief paper is intended as a partial summary of current knowledge of fine particles gathered by the University of Minnesota Particle Technology group both from their own laboratory research and collaborative field studies. Some conclusions from previously published papers are repeated here, but data from these papers is generally excluded. Some new and significant unpublished data is included. Frequent reference is made to two recent papers, Whitby and Liu (1973) and Whitby (1973), which present and discuss much of our most recent knowledge about atmospheric aerosol size distributions and their concentration.

Principal Conclusions About Atmospheric Aerosol Particle size Distributions

Following are some of the principal conclusions we have reached concerning the nature of atmospheric aerosol size distributions. These conclusions have been derived from field study data taken near the earth's surface, predominantly in urban areas.

(1) The mass or volume distribution is usually bimodal with the saddle point in the 1 to $3 \mu \mathrm{m}$ diameter range (see fig. A). The particles larger than a few micrometers originate from natural or man-made 


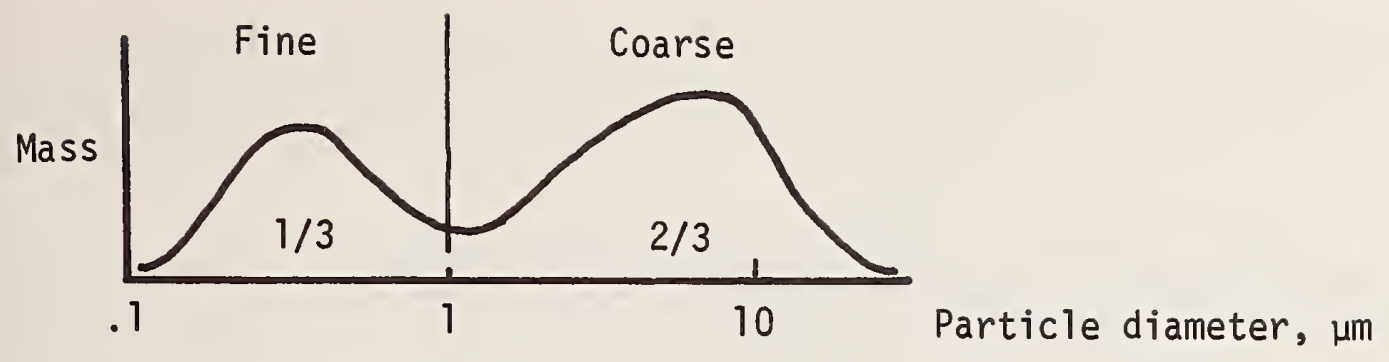

Figure A Atmospheric aerosol mass size distributions are usually bimodal with about one-third of the total mass in the fine particle (or submicrometer) mode and two-thirds in the course particle mode.

mechanical processes. The mechanically produced particles are hereafter called "coarse particles."

The particles smaller than a few micrometers arise predominantly from condensation processes. These aerosols smaller than a few micrometers are called "fine particles." The predominant man-made source of these fine particles is combustion or the condensation of chemical or photochemical reaction products on nuclei from combustion.

The fine particle size range may show two distinct surface area modes (see fig. B). The first mode in the vicinity of $0.02 \mu \mathrm{m}$ diameter results from the direct emission of combustion particles. The second submicrometer mode in the 0.1 to $0.5 \mu \mathrm{m}$ range is the result of either the coagulation of primary particles or the condensation of reaction products or water on primary particles.

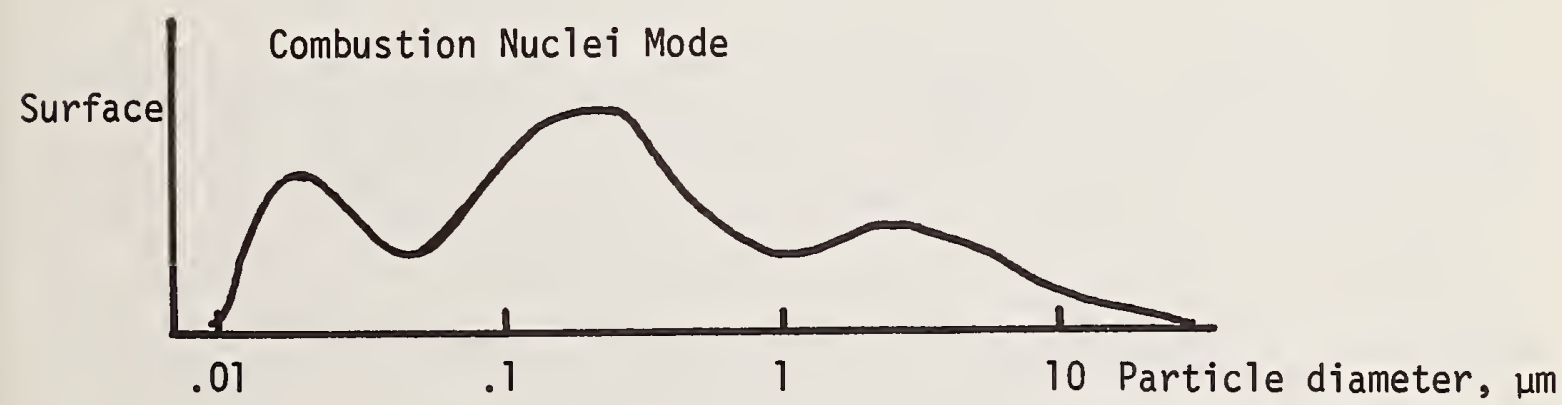

Figure B Atmospheric surface area size distributions may be trimodal near sources of combustion nuclei. 
(2) There appears to be very little exchange of mass under most conditions between the fine and coarse particle ranges in the atmosphere. This means that the origin and behavior of fine particles and coarse particles are different.

(3) Some typical values of the concentration of fine and coarse particles in the atmosphere derived from table 1 (Whitby, 1973) are as follows:

$$
\text { Clean air }=4 \mathrm{\mu m}^{3} / \mathrm{cm}^{3}\left(\rho_{p}=1 \mathrm{~g} / \mathrm{cm}^{3}\right) \text { and coarse particles }=9 \mu \mathrm{g} / \mathrm{m}^{3}
$$

In moderately polluted urban areas, the fine particle concentration is typically $50 \mathrm{\mu g} / \mathrm{m}^{3}$ and coarse particle concentrations are $120 \mathrm{\mu g} / \mathrm{m}^{3}$.

Because of the relative independence of fine and coarse particles, both nonurban and urban sites have yielded widely varying ratios of fine to coarse particles. On the average, however, the fine particles constitute about one-third of the total aerosol mass.

(4) There appears to be a significant difference in the ratio of fine to coarse background aerosol for California, Colorado, and the Eastern part of the United States. The West Coast appears to be lower in large particle concentration than does Colorado. A probable cause is that Colorado is downwind of dry desert areas in which greater quantities of dust are suspended in the atmosphere and transported long distances.

(5) Because of the rapid coagulation rate between $0.01 \mu \mathrm{m}$ size particles and particles in the 0.1 to $0.5 \mu \mathrm{m}$ size range, the Aitken nuclei count under heavily polluted conditions may be lower than when the air is relatively clean. While the Aitken nuclei counter is not a good indicator of general pollution, it may be useful for indicating the proximity of sources of combustion aerosol.

(6) Chemical or photochemical aerosol formation mechanisms may or may not increase the atmospheric particle number depending upon the already existing surface area concentration. At surface area concentrations below about $500 \mu \mathrm{m}^{2} / \mathrm{cm}^{3}$, new nuclei may be formed and will appear in the 0.01 to $0.02 \mu \mathrm{m}$ sjze range. At surface area concentrations greater than a few thousand $\mu \mathrm{m}^{2} / \mathrm{cm}^{3}$, the chemically or photochemically converted material condenses primarily on particles in the size range from 0.1 to $0.5 \mu \mathrm{m}$ and may not form significant numbers of new nuclei.

For example, from some recent aircraft aerosol size distribution measurements taken with the University of Minnesota electrical aerosol analyzer in cooperation with EPA and MRI in St. Louis (September 1973), it was observed that although there was a large amount of secondary aerosol formed in a power plant plume, most of this aerosol mass was deposited on particles in the 0.1 to $0.5 \mu \mathrm{m}$ size range. 
On the other hand, evidence of secondary aerosol formation was observed in the inversions existing at $600 \mathrm{~m}(2000 \mathrm{ft})$ and at $2700 \mathrm{~m}$ (9000 ft.) At $2700 \mathrm{~m}$, because the existing surface area was small, this secondary aerosol formed a mode at $0.02 \mu \mathrm{m}$.

(7) In a recent thesis, Lundgren (1973) reports the results of measurements in which a special set of impactors was used to supplement the Lundgren impactor to measure the mass distribution of aerosol up to $100 \mu \mathrm{m}$ diameter. These data show that the mass distribution is bimodal when measured directly. It also shows that particles up to an aerodynamic diameter of $100 \mu \mathrm{m}$ or more exist under normal conditions in the atmosphere.

Lundgren also found that perhaps 50 percent of the mass of the coarse particles consisted of organic materials such as insect fragments, fibers, and pollen. The remaining coarse particle mass was mineral matter.

Ambient Air Quality Standards

The fact that the origin and behavior of the fine and coarse particles in the atmosphere are independent has important implications for the development of future air quality standards.

Perhaps there should be separate air quality standards for fine and coarse particles, and, correspondingly, separate methods for measuring fine and coarse particles. A study of our data in urban areas also suggests that a greater fraction of the fine particle mass than coarse particle mass is contributed by anthropogenic sources. It appears that in areas where particulate pollution is of any consequence, better than 90 percent of the fine particle mass comes from anthropogenic sources whereas probably not more than one-half or two-thirds of the coarse particles are anthropogenic. Thus the fine particles are a better indicator of anthropogenic contributions to aerosol pollution than are the coarse particles. Total mass as measured by a high volume sampler is of course a combination of the two. However, in general the fine particles represent only one-third or one-half of the total mass. Thus it seems that measurements of fine particle mass would be a better indicator of anthropogenic particulate air pollution than total mass measured by a high volume sampler.

\section{Classification and Names for Fine Particle Aerosol Levels}

Fine particle aerosol concentrations vary greatly in background and urban areas. It has become desirable to make an attempt to classify areas of the world in terms of their fine particle aerosol concentration and to assign names to these conditions and areas. Table 3 shows such a classification. Background conditions have been divided into four categories and urban conditions into three.

The rationale behind this table and supporting data from field research has recently been presented by Whitby et al. (1974). 
Table 3. Background and urban aerosol parameters.

\begin{tabular}{|c|c|c|c|c|}
\hline Type & $\begin{array}{c}\text { Aitken } \\
\text { Number } / \mathrm{cm}^{3} \\
\end{array}$ & $\begin{array}{r}b_{\text {scat }} \\
\times 10^{-4} \mathrm{~m}^{-1} \\
\end{array}$ & $\begin{array}{c}\text { Volume } \\
\text { (submicrometer) } \\
\mu \mathrm{m}^{3} / \mathrm{cm}^{3} \\
\end{array}$ & $\begin{array}{l}\text { Volume } \\
\text { (total) } \\
\mu \mathrm{m}^{3} / \mathrm{cm}^{3} \\
\end{array}$ \\
\hline \multicolumn{5}{|l|}{ Background: } \\
\hline $\begin{array}{l}\text { Oceanic } \\
\text { Clean continental } \\
\text { Average continental } \\
\text { Urban district }\end{array}$ & $\begin{array}{c}100-400 \\
50-1000 \\
2-5 K \\
5-15 K\end{array}$ & $\begin{array}{l}.1-.3 \\
.2-.5 \\
.2-.8 \\
.8-3\end{array}$ & $\begin{aligned} 1 & -4 \\
.5 & -2.5 \\
2.5 & -8 \\
8 & -30\end{aligned}$ & $\begin{array}{r}2-10 \\
10=40 \\
20=60\end{array}$ \\
\hline \multicolumn{5}{|l|}{ Urban } \\
\hline $\begin{array}{l}\text { Clean } \\
\text { Average } \\
\text { Polluted }\end{array}$ & $\begin{array}{l}50-1000 \mathrm{~K} \\
100-200 \mathrm{~K} \\
100-4000 \mathrm{~K}\end{array}$ & $\begin{array}{c}.8-3 \\
3 \\
3-15\end{array}$ & $\begin{array}{c}8-30 \\
35 \\
30-150\end{array}$ & $\begin{aligned} 20 & -60 \\
& 70 \\
100 & -300\end{aligned}$ \\
\hline
\end{tabular}

\section{REFERENCES}

Berglund, R. N. and Liu, B. Y. H. (1973), "Generation of Monodisperse Aerosol Standards," J. of Environmental Science and Technology 7:147153.

Liu, B. Y. H. and Agarwal, J. K. (1973), "Experimental Observation of Aerosol Deposition in Turbulent Flow," submitted to J. of Aerosol Science.

Liu, B. Y. H., Berglund, R. N., and Agarwal, J. K. (1973), "Experimental Studies of Optical Particle Counters," submitted to $\underline{\mathrm{J}}$. of Atmospheric Environment.

Liu, B. Y. H., Marple, V. A., Whitby, K. T., and Barsic, N. J. (1973), "Size Distribution Measurement of Airborne Coal Dust by Optical Particle Counters," Particle Technology Laboratory Pub. No. 183, University of Minnesota.

Liu, B. Y. H. and Pui, D. Y. H. (1973), "A Sub-Micron Aerosol Standard and the Primary, Absolute Calibration of the Condensation Nuclei Counter," accepted by $\underline{J}$. of Colloid and Interface science.

Liu, B. Y. H., Pui, D. Y. H., Hogan, A. W., and Rich, T. A. (1974), "Calibration of the Pollak Counter by Monodisperse Aerosols," submitted to $\mathrm{J}$. of Atmospheric Sciences.

Liu, B. Y. H., Whitby, K. T., and Pui, D. Y. H. (1973), "A Portable Electrical Aerosol Analyzer for Size Distribution Measurement of Submicron Aerosols," presented at the 66th Annual Meeting of the Air Pollution Control Association, Chicago, I11., June 24-28, 1973. 
Whitby, K. T. (1974), "Summary Progress Report for EPA Grant No. R801175, Generation and Decay of Sma11 Ions and EPA Grant No. R800971, Sampling and Analysis of Atmospheric Aerosols, "Particle Technology Laboratory Pub. No. 228.

Whitby, K. T. and Clark, W. E. (1966), "Electric Aerosol Particle Counting and Size Distribution Measuring System for the 0.015 to 1 Micron Size Range," Tel1us 18:573-586.

Whitby, K. T., Clark, W. E., Marple, V. A., Sverdrup, G. M., Willeke, K., Liu, B. Y. H., and Pui, D. Y. H. (1973), "Evolution of the Freeway Aerosol," presented at the Symposium on Surface and Colloid Chemistry in Air Pollution Control, ACS Annual Meeting, Chicago, I11., Aug. 29, 1973.

Whitby, K. T. and Liu, B. Y. H. (1973), "Advances in Instrumentation and Techniques for Aerosol Generation and Measurement," presented at the Harold Heywood Memorial Symposium, Loughborough, England, Sept. 17-18, 1973.

Whitby, K. T., Liu, B. Y. H., Husar, R. B., and Barsic, N. J. (1972), "The Minnesota Aerosol Analyzing System," J. of Colloid and Interface Science 39:136-164.

Whitby, K. T., "On the Multimodal Nature of Atmospheric Aerosol Size Distributions," presented at the VIII International Conference on $\mathrm{Nu}-$ cleation, Leningrad, U.S.S.R., Sept. 24-28, 1973.

Lundgren, D. A., "Mass Distribution of Large Atmospheric Particles," Ph.D. Thesis, University of Minnesota (1973).

Whitby, K. T., Pui, D. Y. H., Sverdrup, G., and Clark, W. E., "Background Aerosol Size Distributions," presented at the 16th Annual Meeting of the American Chemical Society, Los Angeles, Calif., Apr. 1, 1974.

\section{DISCUSSION}

GEORGE SINNOTT: It seems to me that in the calibration of your electronic analyzer, you made the same measurement twice on a particle with different results. You run through essentially a mobility analyzer and run through the portable analyzer and get two complete sets. The monodisperse aerosol is simply the results of the mobility analyzer?

MARPLE: Dr. Sinnott's question concerns the validity of the calibration procedure in which an aerosol size analyzer operating on the principle of particle electrical mobility is calibrated by a monodisperse aerosol whose size is also determined from electrical mobility. 
It is true that both the monodisperse aerosol generator and the aerosol analyzer make use of the electrical mobility of the particles; there are certain differences that should be noted.

In the monodisperse aerosol generator, particles carrying one elementary unit $\left(4.8 \times 10^{-10}\right.$ esu) of charge are used. Since the particle charge is known precisely in this case, the particle size can be determined precisely by measuring the particle electrical mobility (see eq 4). Similarly the absolute particle concentration can be determined by collecting the particles and measuring the current and flow (see eq 6). on the other hand, the electrical aerosol analyzer makes use of a unipolar diffusion charger which places an unknown charge on the particles. This charge must be determined experimentally as a function of particle size. Further, the particle loss in the system due to diffusion must also be determined experimentally. The sub-micron aerosol generator provides an excellent source of uniform particles of known size and concentration for these experimental purposes.

While the size and concentration of the monodisperse aerosols were calculated on the basis of electrical measurements, they have been checked against an independent manual technique involving sampling the particles, and sizing and counting them in the electron microscope. Very good agreement has been obtained. We estimate the sizing and concentration accuracies of the electrical method to be 2 percent and 5 percent respectively.

EDGAR ETZ: I noticed that your inorganic particles you produced were certainly not spherical. Are there ways of producing spherical particles of inorganic substances? Have you tried to go this way?

MARPLE: The sodium chloride particles generated on the vibrating orifice generator (fig. 4 (C) of paper) were not spherical. However, the methylene blue particles (fig. 4 (a) of paper) are spherical.

ETZ: I would think that it has to do with the nature of the material that produces the particle.

MARPLE: Do you mean the shape of the particle?

ETZ: The shape of the particles coming from the aerosol.

MARPLE: The sodium chloride particles were crystals and the methylene blue particles were spheres.

ETZ: You apply them to a nigh temperature flame, and in that process none of them may come out as spheres.

MILTON KERKER: Dr. Etz, all of your colleagues from Clarkston were making particles from inorganic compounds.

ETZ: Out of ionic materials? 
KERKER: Sodium chloride, vanadium pentoxide, silver chloride. Yes, you are quite right, you can make them in a flame; or you can just simply sublime them and cool them and you have beautifully spherical particles.

SINNOTT: Dr. Kerker, was that the same size range you were talking about--27 micrometers?

KERKER: On these were smaller, but I don't see why we couldn't.

LARRY DOEMENY: 27 micrometers?

KERKER: We haven't made them that large, yet.

MADHAV RANADE: We made some at the place I was working. We were using a vibrating jet using molten liquids, molten sodium chloride, and forcing it out under high pressure through a small orifice, and by that method we could produce spherical particles.

ETZ: To what extent were they spherical? Were they crystallizing on the surface of the sphere or what?

RANADE: It depends on the temperature. If they are hot enough, the surface of the sphere solidified first, and essentially that is preserved, but if they cooled slowly, then they had a chance to develop crystallinity. I don't exactly off-hand know what the temperatures would have to be.

RAYMOND CHUAN: These aluminum oxide spheres coming out of a combustion process are very spherical. They go down to about 10-12 $\mu \mathrm{m}$.

ETZ: Can you take time to describe briefly your coal dust generally? What makes the coal dust particle not an ideal particle to measure?

MARPLE: A nonideal particle as I described it is light absorbing, and nonspherical. It is very irregular in shape.

ETZ: How did you produce it in your generator?

MARPLE: We used a fluidized aerosol generator. As shown in the figure (fig. 10 of paper) the fluidized bed was obtained by placing approximately $3 \mathrm{~cm}$ of bronze powder (100 $\mathrm{mm}$ diameter spheres) over a supporting screen in a $7.5 \mathrm{~cm} \mathrm{I.D.} \mathrm{aluminum} \mathrm{tube} \mathrm{and} \mathrm{flowing} \mathrm{clean} \mathrm{fil-}$ tered air vertically upward through the column at the rate of 10 LPM. The air was dried to avoid oxidation of the bed material. Finely ground coal was placed in a small tube and introduced into the bottom of the fluidized bed by a short burst of air. Microscopic examination of the coal dust generated by this method showed that deagglomeration was thorough and complete even for submicron particles which are difficult 
to deagglomerate by conventional methods such as aerodynamic impaction or turbulent dispersion.

ETZ: Why did you use amorphous materials?

MARPLE: Coal particles were used on the calibrations material because that is the material of the particles which the optical particle counter was to measure. Generally, the optical particle counter calibration curve is dependent on the refractive index and shape of the particles being sized. Our calibrations were made with coal dust produced in a ball mill so the particles were modular in shape. However, when we examined coal dust as produced at a cutting tool we found that the particles were spears, platelets, and very irregular in shape. 
Speakers

I lan Chabay

Department of Chemistry

University of Illinois

Urbana, IL 61801

R. Chuan, Staff Assistant

Celesco Industries Inc.

Environmental and Industrial Products Costa Mesa, CA 92626

Reg Davies

IIT Research Institute

Chicago, IL 60116

Laurence Doemeny

Engineering Branch

Division of Laboratories and NIOSH Criteria Development

Cincinnati, OH 45202

Cary Gravatt

Office of Associate Director for Programs

National Bureau of Standards

Washington, DC 20234

Geoffrey Hotham, President

Laser Holography Inc.

Santa Barbara, CA 93108

Mi1ton Kerker, Dean

The School of Arts and Sciences

Clarkson College of Technology

Potsdam, NY 13676

Robert Knollenberg, President

Particle Measuring Systems, Inc.

5469 Western Avenue

Boulder, CO 80301

Albert McSweeney, Research Physicist

Experiment Station

Georgia -Institute of Technology

Atlanta, GA 30332
Virgil Marple

Department of Mechanical Engineering

125 Mechanical Engineering Building

University of Minnesota

Minneapolis, MN 55455

Charles Parmenter

Department of Chemistry

Indiana University

Bloomington, IN 47401

M. Ranade, Research Chemical Engineer

IIT Research Institute

Chicago, IL 60116

William Yanta

Aerophysics Division

Naval Ordnance Laboratory

Silver Spring, MD 20910

\section{Other Attendees}

Wendel1 Anderson, Head

FC Division

U.S. Naval Weapons Laboratory

Dahlgren, VA 22448

George Carson, Chief

Personal and Environmental Air

Measurements Section

Center for Disease Control, NIOSH

Cincinnati, $\mathrm{OH} 45202$

Wayne Cassatt, Coordinator

Particle Technology Group

Surface Microanalysis Section

Analytical Chemistry Division

National Bureau of Standards

Washington, DC 20234

Robert Coon

Bureau of Foods

Food and Drug Administration

Washington, DC 20204 
Heinz Eiermann, Acting Director Division of Cosmetics Technology Food and Drug Administration 200 C Street, SW

Washington, DC 20204

\section{Edgar Etz}

Particle Technology Group

Surface Microanalysis Section Analytical Chemistry Division National Bureau of Standards Washington, DC 20234

Kurt Heinrich, Chief

Surface Microanalysis Section Analytical Chemistry Division National Bureau of Standards Washington, DC 20234

William Kirchhoff

Measures for Air Quality Program Institute for Materials Research National Bureau of Standards Washington, DC 20234

Albert Kolbye, Jr., Acting Director Office of Sciences

Food and Drug Administration 200 C Street, SW Washington, DC 20204

Philip LaFleur, Acting Chief Analytical Chemistry Division National Bureau of Standards Washington, DC 20234

James McNesby

Measures for Air Quality Program Institute for Materials Research National Bureau of Standards Washington, DC 20234

Bernard McNammarraa Toxicology Division Biomedical Laboratory Edgewood Arsenal, MD 21010
Ronald Nelson

Chemistry and Physics Laboratory Environmental Protection Agency Research Triangle Park, NC 27709

Edmond Owens, Chief

Environmental Toxicology Branch

Toxicology Division

Biomedical Laboratory

Edgewood Arsenal, MD 21010

George Sinnott, Acting Chief

Program for Physics and Dynamics

Fire Technology Division

Institute for Applied Technology

National Bureau of Standards

Washington, DC 20234

George Thompson

Division of Cosmetics Technology Food and Drug Administration 200 C Street, SW

Washington, DC 20204

Jack Weimer, Assistant Chief

Environmental Toxicology Branch

Toxicology Division

Biomedical Laboratory

Edgewood Arsena 1, MD 21010

Gale Wyer, Director

Division of Chemistry

Bureau of Biomedical Science

Consumer Product Safety Commission

200 C Street, SW

Washington, DC 20204

Robert Williamson, Jr. Naval Ordnance Laboratory Silver Spring, MD 20910 


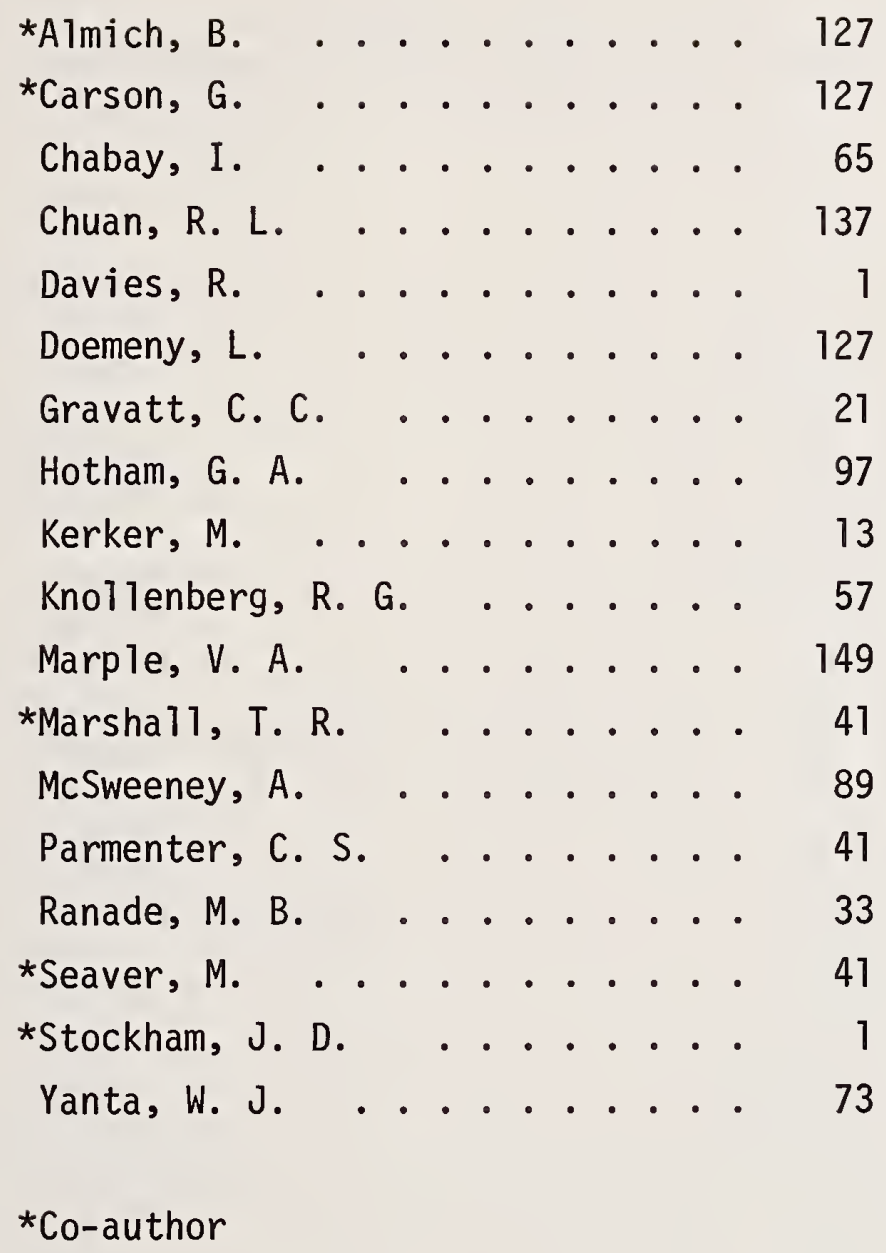


Entries were taken from the key words given by authors of the papers printed herein. Reference is to the person who presented the paper at the seminar. Although this index is not exhaustive, it should serve as a subject guide.

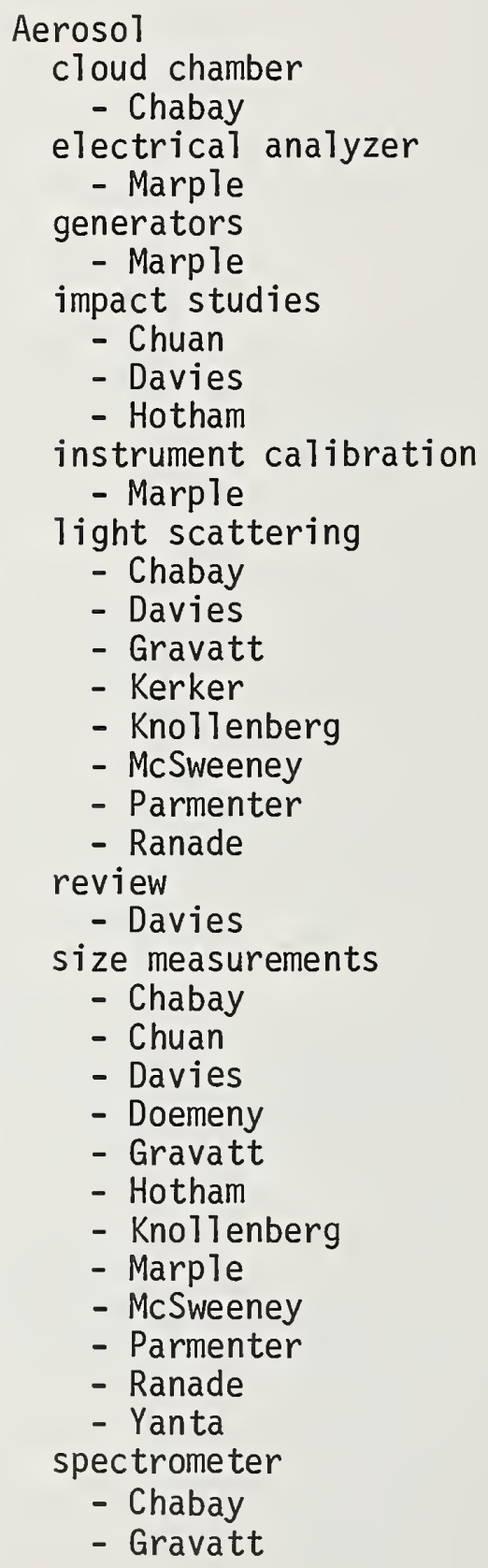

- Kerker

- Knollenberg

- McSweeney

- Parmenter

- Ranade

review

- Davies

size measurements

- Chabay

- Chuan

- Davies

- Doemeny

- Gravatt

- Hotham

- Knollenberg

- Marple

- McSweeney

- Parmenter

- Ranade

- Yanta

spectrometer

- Chabay

- Gravatt

- Hotham

- Knollenberg

- Marple

- McSweeney

- Parmenter

- Ranade

- Yanta

sprays

- Davies

- Hotham

- Parmenter

- Ranade

- Yanta

therapeutic

- Ranade

Beta absorption

- Doemeny

Cascade impactor

- Chuan

Coal dust monitor

- Doemeny

Detection of particles by impaction

- Chuan

Doppler measurements of particle size

- Chabay

- Yanta

Droplet

cloud measurements

- Chabay

- Knollenberg

condensation on

- Ranade

evaporation of

- Ranade

imaging

- Davies

-Hotham

- Knollenberg 


$$
\begin{aligned}
& \text { sizing } \\
& \text { - Davies } \\
& \text { - Hotham } \\
& \text { - Yanta }
\end{aligned}
$$

Dust inhalation hazards

- Doemeny

Electrical mobility

- Davies

- Marple

Environmental sampler

Fibers

- Doemeny

- Kerker

Interferometer

- Knollenberg

- Yanta

Laser heterodyne

- Chabay

Laser holography

- Hotham

Laser imaging of particles

- Davies

- Hotham

- Knollenberg

Laser light scattering by aerosols

- Chabay

- Davies

- Gravatt

- Knollenberg

- McSweeney

- Parmenter

- Ranade

- Yanta

scattering diagrams

- McSweeney

- Parmenter

Momentum methods

- Davies

Nuclei counters

- Marple

Optical transform

- McSweeney

Particle

chemical characterization

- Gravatt

cloud chamber

- Chabay electrical analyzer

- Marple

fire produced

- Gravatt

generators

- Marple

imaging

- Davies

- Hotham

- Knollenberg

impact studies

- Chuan

- Davies

- Hotham

instrument calibration

- Marple

light scattering

- Chabay

- Davies

- Gravatt

- Kerker

- Knollenberg

- McSweeney

- Parmenter

- Ranade

refractive index

- Gravatt

- Kerker

- Parmenter

review

- Davies

size measurements

- Chabay

- Chuan

- Davies

- Doemeny

- Gravatt

- Hotham

- Knollenberg

- Marple

- McSweeney

- Parmenter

- Ranade

- Yanta

spectrometer

- Chabay

- Gravatt

- Hotham 
- Knollenberg

- Marple

- McSweeney

- Parmenter

- Ranade

- Yanta

standards

- Marple

therapeutic

- Ranade

$360^{\circ}$ scattering by

- Parmenter

velocity measurements

- Yanta

Particulates (see particles)

Piezoelectric crystal

- Chuan

Quartz crystal microscope

- Chuan

Respirable dust sampler

- Doemeny

Smoke detector

- Gravatt 


\begin{tabular}{|c|c|c|}
\hline $\begin{array}{l}\text { U.S. DEPT. OF COMM. } \\
\text { BIBLIOGRAPHIC DATA } \\
\text { SHEET }\end{array}$ & \begin{tabular}{|l|l|} 
1. PUBLICATION OR REPORT NO. & $\begin{array}{l}\text { 2. Gov't Accession } \\
\text { No. }\end{array}$ \\
NBS Special Publication 412 & \\
\end{tabular} & 3. Recipient's Accession No. \\
\hline \multicolumn{2}{|l|}{ 4. TITLE AND SUBTITLE } & 5. Publication Date \\
\hline \multirow{2}{*}{\multicolumn{2}{|c|}{$\begin{array}{l}\text { AEROSOL MEASUREMENTS, PROCEEDINGS OF SEMINAR } \\
\text { ON AEROSOL MEASUREMENTS, MAY } 7,1974\end{array}$}} & October 1974 \\
\hline & & 6. Performing Organization Code \\
\hline \multicolumn{2}{|c|}{ 7.AUTHOR(S) W. A. Cassatt and R. S. Maddock, editors } & 8. Performing Organ. Report No. \\
\hline \multirow{2}{*}{\multicolumn{2}{|c|}{$\begin{array}{l}\text { 9. PERFORMING ORGANIZATION NAME AND ADDRESS } \\
\text { NATIONAL BUREAU OF STANDARDS } \\
\text { DEPARTMENT OF COMMERCE } \\
\text { WASHINGTON, D.C. } 20234\end{array}$}} & 10. Project/Task/Work Unit No. \\
\hline & & 11. Contract/Grant No. \\
\hline \multirow{2}{*}{\multicolumn{2}{|c|}{$\begin{array}{l}\text { 12. Sponsoring Organization Name and Complete Address (Street, City, State, ZIP) } \\
\text { Nationa1 Bureau of Standards } \\
\text { Food and Drug Administration, Washington, D. C. }\end{array}$}} & $\begin{array}{l}\text { 13. Type of Report \& Period } \\
\text { Covered }\end{array}$ \\
\hline & & \\
\hline
\end{tabular}

\section{SUPPLEMENTARY NOTES}

Library of Congress Catalog Number: 74-600164

16. ABSTRACT (A 200-word or less factual summary of most significant information. If document includes a significant

bipliography or fiterature sugvey, mention it here.)

Papers followed by discussions were given in a closed seminar and workshop sponsored by the National Bureau of Standards and the Food and Drug Administration to define the state of development of aerosol measuring instruments. The instruments discussed were based upon a variety of operating principles including laser light scattering, optical imaging, Doppler shift, electromobility, piezoelectric effect, and beta-ray absorption. Two review papers were given which described other phenomena upon which aerosol measurements are based. The general summary includes a table which lists the specifications of the instruments discussed to illustrate the range of capabilities available in this field. Discussion among seminar attendees revealed that many questions remain to be answerec before the more difficult aerosol measurements problems can be solved. For example, in the analysis of very dense aerosols questions arise concerning coincidence losses or agglomeration effects that may result from collisions between particles as they are drawn into the measuring volume. Volatilization or condensation effects may alter the size distribution if the measurements are made late in time. Finally, variations in particle shape or index of refraction can alter the instrument response and cause difficulties in interpretation.

17. KEY WORDS (six to twelve entries; alphabetical order; capitalize only the first letter of the first key word unless a proper name; separated by semicolons)

Aeroso1 instrument performance; aerosol measuring instruments; beta-ray absorption; Doppler shift; electromobility; 1aser light scattering; ontical imaging; piezoelectric effect

\begin{tabular}{|c|c|c|}
\hline $\begin{array}{l}\text { 18. AVAILABILITY } \\
\square \text { For Official Distribution. Do Not Release to NTIS }\end{array}$ & $\begin{array}{l}\text { 19. SECURITY CLASS } \\
\text { (THIS REPURT) } \\
\text { UNCL ASSIF IED }\end{array}$ & $\begin{array}{l}\text { 21. NO. OF PAGES } \\
182 \\
412\end{array}$ \\
\hline $\begin{array}{l}\text { X. Order From Sup. of Doc., U.S. Government Printing Office } \\
\text { Washington, D.C. 20402, SD Cat. No. C13.10:412 }\end{array}$ & $\begin{array}{l}\text { 20. SECURITY CLASS } \\
\text { (THIS PAGE) }\end{array}$ & 22. Price \\
\hline $\begin{array}{l}\text { Order From National Technical Information Service (NTIS) } \\
\text { Springfield, Virginia } 22151\end{array}$ & UNCLASSIFIED & $\$ 2.05$ \\
\hline
\end{tabular}







\section{NBS TECHNICAL PUBLICATIONS}

\section{PERIODICALS}

JOURNAL OF RESEARCH reports National Bureau of Standards research and development in physics, mathematics, and chemistry. Comprehensive scientific papers give complete details of the work, including laboratory data, experimental procedures, and theoretical and mathematical analyses. Illustrated with photographs, drawings, and charts. Includes listings of other NBS papers as issued.

Published in two sections, available separately:

- Physics and Chemistry (Section A)

Papers of interest primarily to scientists working in these fields. This section covers a broad range of physical and chemical research, with major emphasis on standards of physical measurcment, fundamental constants, and properties of matter. Issued six times a year. Annual subscription: Domestic, $\$ 17.00$; Foreign, $\$ 21.25$.

\section{- Mathematical Sciences (Section B)}

Studies and compilations designed mainly for the mathematician and theoretical physicist. Topics in mathematical statistics, theory of experiment design, numerical analysis, theoretical physics and chemistry, logical design and programming of computers and computer systems. Short numerical tables. Issued quarterly. Annual subscription: Domestic, $\$ 9.00$; Foreign, $\$ 11.25$.

DIMENSIONS/NBS (formerly Technical News Bulletin)-This monthly magazine is published to inform scientists, engineers, businessmen, industry, teachers, students, and consumers of the latest advances in science and technology, with primary emphasis on the work at NBS.

DIMENSIONS/NBS highlights and reviews such issues as energy research, fire protection, building technology, metric conversion, pollution abatement, health and safety, and consumer product performance. In addition, DIMENSIONS/NBS reports the results of Bureau programs in measurement standards and techniques, properties of matter and materials, engineering standards and services, instrumentation, and automatic data processing.

Annual subscription: Domestic, $\$ 6.50$; Foreign, $\$ 8.25$.

\section{NONPERIODICALS}

Monographs-Major contributions to the technical literature on various subjects related to the Bureau's scientific and technical activities.

Handbooks-Recommended codes of engineering and industrial practice (including safety codes) developed in cooperation with interested industries, professional organizations, and regulatory bodies.

Special Publications-Include proceedings of high-level national and international conferences sponsored by NBS, precision measurement and calibration volumes, NBS annual reports, and other special publications appropriate to this grouping such as wall charts and bibliographies.

Applied Mathematics Series-Mathematical tables, manuals, and studies of special interest to physicists, engineers, chemists, biologists, mathematicians, computer programmers, and others engaged in scientific and technical work.
National Standard Reference Data Series-Provides quantitative data on the physical and chemical properties of materials, compiled from the world's literature and critically evaluated. Developed under a world-wide program coordinated by NBS. Program under authority of National Standard Data Act (Public Law 90-396). See also Section 1.2.3.

Building Science Series-Disseminates technical information developed at the Bureau on building materials, components, systems, and whole structures. The series presents research results, test methods, and performance criteria related to the structural and environmental functions and the durability and safety characteristics of building elements and systems.

Technical Notes-Studies or reports which are complete in themselves but restrictive in their treatment of a subject. Analogous to monographs but not so comprehensive in scope or definitive in treatment of the subject area. Often serve as a vehicle for final reports of work performed at NBS under the sponsorship of other government agencies.

Voluntary Product Standards-Developed under procedures published by the Department of Commerce in Part 10, Title 15, of the Code of Federal Regulations. The purpose of the standards is to establish nationally recognized requirements for products, and to provide all concerned interests with a basis for common understanding of the characteristics of the products. The National Bureau of Standards administers the Voluntary Product Standards program as a supplement to the activities of the private sector standardizing organizations.

Federal Information Processing Standards Publications (FIPS PUBS)-Publications in this series collectively constitute the Federal Information Processing Stand ards Register. The purpose of the Register is to serve as the official source of information in the Federal Government regarding standards issued by NBS pursuant to the Federal Property and Administrative Services Act of 1949 as amended, Public Law 89-306 (79 Stat. 1127), and as implemented by Executive Order 11717 (38 FR 12315, dated May 11, 1973) and Part 6 of Title 15 CFR (Code of Federal Regulations). FIPS PUBS will include approved Federal information processing standards information of general interest, and a complete index of relevant standards publications.

Consumer Information Series-Practical information, based on NBS research and experience, covering areas of interest to the consumer. Easily understandable language and illustrations provide useful background knowledge for shopping in today's technological marketplace.

NBS Interagency Reports-A special series of interim or final reports on work performed by NBS for outside sponsors (both government and non-government). In general, initial distribution is handled by the sponsor; public distribution is by the National Technical Information Service (Springfield, Va. 22151) in paper copy or microfiche form.

Order NBS publications (except Bibliographic Subscription Services) from: Superintendent of Documents, Government Printing Office, Washington, D.C. 20402.

\section{BIBLIOGRAPHIC SUBSCRIPTION SERVICES}

The following current-awareness and literature-survey bibliographies are issued periodically by the Bureau:

Cryogenic Data Center Current Awareness Service (Publications and Reports of Interest in Cryogenics). A literature survey issued weekly. Annual subscription: Domestic, $\$ 20.00$; foreign, $\$ 25.00$.

Liquefied Natural Gas. A literature survey issued quarterly. Annual subscription: $\$ 20.00$.

Superconducting Devices and Materials. A literature survey issued quarterly. Annual subscription: $\$ 20.00$. Send subscription orders and remittances for the pre- ceding bibliographic services to the U.S. Department of Commerce, National Technical Information Service, Springfield, Va. 22151.

Electromagnetic Metrology Current Awareness Service (Abstracts of Selected Articles on Measurement Techniques and Standards of Electromagnetic Quantities from D-C to Millimeter-Wave Frequencies). Issued monthly. Annual subscription: $\$ 100.00$ (Special rates for multi-subscriptions). Send subscription order and remittance to the Electromagnetic Metrology Information Center, Electromagnetics Division, National Bureau of Standards, Boulder, Colo, 80302 
U.S. DEPARTMENT OF COMMERCE

National Bureau of Standards

Washington, D.C. 20234

POSTAGE AND FEES PAID

DFFICIAL BUSINESS U.S. DEPARTMENT OF COMMERCE $C O M-215$

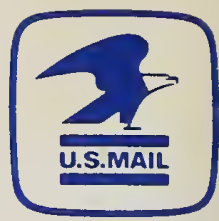

Penalty for Private Use, $\$ 300$

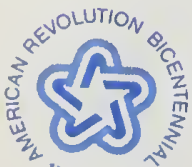

1776-1976 